\title{
Effective JSJ Decompositions
}

\author{
Olga Kharlampovich and Alexei Myasnikov
}

\begin{abstract}
In this paper we describe an elimination process which is a deterministic rewriting procedure that on each elementary step transforms one system of equations over free groups into a finitely many new ones. Infinite branches of this process correspond to cyclic splittings of the coordinate group of the initial system of equations. This allows us to construct algorithmically Grushko's decompositions of finitely generated fully residually free groups and cyclic [abelian] JSJ decompositions of freely indecomposable finitely generated fully residually free groups. We apply these results to obtain an effective description of the set of homomorphisms from a given finitely presented group into a free group, or, more generally, into an NTQ group.
\end{abstract}

\section{CONTEnTS}

Introduction 2

1. Preliminaries 8

2. Splittings 15

3. Algorithms over fullv residually free groups 34

4. Generalized equations over free groups 39

5. Elimination process: construction of $T(\Omega) \quad 48$

6. Elimination process: periodic structures 64

7. Elimination process: splittings of coordinate groups 79

8. Structure of solutions. the solution tree $T_{\mathrm{col}}(\Omega . \Lambda) \quad 90$

9. Maximal standard quotients and canonical embeddings of $\mathcal{F}$-groups 93

10. Effective free decompositions 101

11. Homomorphisms of finitely generated groups into fully residually free groups 106

12. Free Lvndon length functions on NTQ groups. 108

13. Effective construction of JSJ decompositions of groups from $\mathcal{F}$. 113

14. Homomorphisms into NTQ groups 121

15. Some applications to equations in $\mathcal{F}$-groups 122

$\begin{array}{ll}\text { References } & 124\end{array}$

1991 Mathematics Subject Classification. Primary 20F10; Secondary 03C05.

Key words and phrases. Free group, splittings, Bass-Serre theory, algorithms.

The first author was supported by NSERC Grant .

The second author was supported by NSERC Grant and by NSF GrantDMS-9970618.

(C)0000 (copyright holder) 


\section{Introduction}

0.1. JSJ decompositions: a bit of history and intentions. A splitting of a group $G$ is a presentation of $G$ as the fundamental group of a graph of groups. According to Bass-Serre theory, a group has a non-trivial splitting if and only if it acts non-trivially on a tree. A splitting is cyclic [abelian] if all edge groups are cyclic [abelian]. In 63 Sela introduced universal cyclic splittings of a particular type, so called JSJ decompositions, which contain (in an appropriate sense) all other cyclic splittings of the group. Such splittings are analogues to the characteristic submanifold constructions for irreducible 3-manifolds described by Jaco and Shalen 31 and Johannson [30, hence the name. Sela showed that JSJ decompositions exist for freely indecomposable torsion-free hyperbolic groups. Existence of JSJ decompositions for arbitrary finitely presented groups was shown by Rips and Sela in 67, their method is based on group actions on $\mathbb{R}$-trees. A different way to obtain JSJ splittings of one-ended hyperbolic groups was proposed by Bowditch [10, he used the local cut point structures on the boundaries. More general approaches to JSJ decompositions of groups have been described by Dunwoody and Sageev 19] and by Fujiwara and Papasoglu 22. They use tracks on 2-complexes and actions on products of trees, correspondingly. All these results prove the existence of JSJ decompositions in various classes of groups, but do not give an algorithm to construct them.

In this paper we algorithmically construct Grushko's decompositions of finitely generated fully residually free groups and cyclic [abelian] JSJ decompositions of freely indecomposable finitely generated fully residually free groups. We apply these results to obtain an effective description of the set of homomorphisms from a given finitely presented group into a free group, or, more generally, into an NTQ group.

Non-trivial JSJ decompositions of a given group $G$ are very closely related with automorphisms of $G$. Originally, they were used mostly to describe the automorphism group $A u t(G)$ of $G$ 63. It turned out recently, that various types of JSJ splittings are very useful in studying equations over groups and, more generally, elementary theories of groups. In particular, we show here that such splittings provide a group theoretic counterpart to various pieces of Makanin-Razborov process and give algebraic semantics to our elimination rewriting process from [36. To explain the main idea of our approach to JSJ we need some notation and definitions.

0.2. Three approaches to fully residually free groups. Let $F=F(A)$ be a free group with basis $A$. Fully residually free groups, which are also known as freely discriminated groups $[\mathbf{4}$, and $\omega$-residually free groups $\mathbf{6 6}$, play a crucial role in the theory of equations and model theory of free groups. Recall that a group $G$ is discriminated by $F$ (or freely discriminated) if for any finite subset $K \subseteq G$ of non-trivial elements there exists a homomorphism $\phi: G \rightarrow F$ such that $g^{\phi} \neq 1$ for each $g \in K$. We describe these groups in Subsection 1.4 and discuss their properties in Subsection 1.5] It is worthwhile to mention here that finitely generated freely discriminated groups can be viewed in three different ways each of which gives one essentially different tools to deal with them. First, they appear as coordinate groups of irreducible systems of equations over free groups, so methods of algebraic geometry over groups can be used here, as well as Makanin-Razborov's techniques; secondly, they can be obtained from free groups by finitely many free products 
with amalgamation and HNN extensions of a very particular type, hence one can easily apply methods of Bass-Serre theory; thirdly, these groups can be faithfully presented by infinite words over abelian ordered groups $\mathbb{Z}^{n}$, which provides one with various cancellation techniques, Nielsen method, and Stallings' foldings apparatus to study their finitely generated subgroups. We frequently use all these techniques below. Notice, that finitely generated fully residually free groups have some other interesting characterizations. Remeslennikov showed that a finitely generated group $H$ is fully residually free if and only if $H$ has exactly the same universal theory as $F$ 66. Recently Sela described finitely generated fully residually free groups precisely as the limit groups 69. Champetier and Guirardel gave another characterization of these groups as limits of free groups in a compact space of marked groups [14.

Now we describe briefly the major components of our method.

0.2.1. Fully residually free groups as coordinate groups. We start with a brief description of a few notions from algebraic geometry over groups. For a detailed discussion on the subject we refer to [4] and [34, see also Section 1.3 By $V_{F}(S)$ we denote the set of all solutions of the system $S(X, A)=1$ in $F$ (the algebraic set defined by $S$ ). The algebraic set $V_{G}(S)$ uniquely corresponds to the normal subgroup (the radical of $S$ ):

$$
R(S)=\left\{T(X) \in F(A \cup X) \quad \forall A \in V_{F}(S)(T(A)=1)\right\}
$$

of the free group $F(A \cup X)$. The quotient group

$$
F_{R(S)}=F(A \cup X) / R(S)
$$

is the coordinate group of the algebraic set $V(S)$. Observe, that if $V_{F}(S) \neq \emptyset$ then $F$ is a subgroup of $F_{R(S)}$. It has been shown in 4 that algebraic sets $V_{F}\left(S_{1}\right)$ and $V_{F}\left(S_{2}\right)$ are rationally equivalent if and only if there exists an isomorphism between their coordinate groups which is identical on $F$. One can define Zariski topology on $F^{m}(m \in \mathbb{N})$ taking algebraic sets as the closed subsets. Guba proved in [27] that free groups are equationally Noetherian, i.e., this Zariski topology is Noetherian , so every closed set is a finite union of irreducible components. It turned out 4. that an algebraic set is irreducible if and only if its coordinate group is fully residually free. Furthermore, every finitely generated fully residually free group can be realized as the coordinate group of a finite system of equations over $F$. From the group theoretic view-point the elimination process mentioned above is all about the coordinate groups of the systems involved. This allows one to transform pure combinatorial and algorithmic results obtained in the process into statements about fully residually free groups.

0.2.2. Fully residually free groups as fundamental groups of graphs of groups. In order to describe solutions of equations in free groups Lyndon introduced the notion of a group with parametric exponents in an associative unitary ring $A$ [47. In particular, he described and studied free exponential groups $F^{\mathbb{Z}[t]}$ over a ring of polynomials $\mathbb{Z}[t]$. One of the principle outcomes of his study is that the group $F^{\mathbb{Z}[t]}$ is discriminated by $F$. A modern treatment of exponential groups is due to Myasnikov and Remeslennikov [55. They showed, in particular, that the group $F^{Z[t]}$ can be obtained as union of an infinite chain, which starts at $F$, of HNNextensions of a very specific type - extensions of centralizers (see Subsection 1.4). This implies that a finitely generated subgroup of $F^{Z[t]}$ is a subgroup of a group which can be obtained from $F$ by finitely many extensions of centralizers, so one can apply Bass-Serre theory to describe the structure of these subgroups. 
We proved in 36 that finitely generated fully residually free groups are embeddable into $F^{Z[t]}$. This, in view of the Lyndon's result mentioned above, gives a complete characterization of such groups. It follows from Bass-Serre theory now that all these groups, except for abelian ones, admit an essential cyclic splitting.

0.2.3. Fully residually free groups via infinite words. Another key component of our approach to finitely generated fully residually free groups is based on Lyndon's length functions and infinite words. To axiomatize Nielsen cancellation argument Lyndon introduced in 48 abstract length functions on groups (now called Lyndon's length functions). He showed that a group $G$ with a free Lyndon's length function with values in $\mathbb{Z}$ (viewed as ordered abelian group) is embeddable into a free group (represented by finite reduced words with natural length function) and the embedding preserves the length, hence $G$ is free. It turned out that a similar result holds for finitely generated fully residually free groups. Namely, it has been shown in $\left[53\right.$ that elements of the free Lyndon's group $F^{\mathbb{Z}[t]}$ can be represented by infinite words in the alphabet $X^{ \pm 1}$. These words are functions of the type

$$
w:\left[1, \alpha_{w}\right] \rightarrow X^{ \pm 1}
$$

where $\alpha_{w} \in \mathbb{Z}[t]$ and $\left[1, \alpha_{w}\right]=\left\{g \in \mathbb{Z}[t] \mid 1 \leq g \leq \alpha_{w}\right\}$ is a closed interval in $\mathbb{Z}[t]$ with respect to the standard lexicographical order $\leq$. The function $L: w \rightarrow \alpha_{w}$ gives rise to a regular free Lyndon length function on $F^{\mathbb{Z}[t]}$ with values in the additive group of $\mathbb{Z}[t]$, viewed as an abelian ordered group. This implies that every finitely generated fully residually free group has a free length function with values in a free abelian group $\mathbb{Z}^{n}$ of finite rank with the lexicographic order. Once a presentation of elements of $F^{\mathbb{Z}[t]}$ by infinite words is established, a host of problems about $F^{\mathbb{Z}[t]}$ can be solved precisely in the same way as in the standard free group $F$. In particular, if $H$ is a finitely generated subgroup of a finitely generated fully residually free group $G$, then one can effectively embed $G$ (hence $H$ ) into $F^{\mathbb{Z}[t]}$. Now using the representation above of elements of $G$ by infinite words one can algorithmically construct a finite labelled graph $\Gamma_{H}$ (finite automata) which accepts precisely elements of $H$ (given by their canonical forms in $F^{\mathbb{Z}[t]}$ ) (see [57]). The process of constructing the graph $\Gamma_{H}$ is a direct generalization of the Stallings' folding procedure, but on infinite words. This allows one to treat finitely generated subgroups of fully residually free groups precisely in the same manner as in the standard free groups. This approach was realized in [46] where a host of algorithmic problems about finitely generated subgroups of finitely generated fully residually free groups was solved (see also Section [3.2).

0.3. Main ideas: elimination process vs JSJ. Our main tool in constructing JSJ decompositions of groups is a so-called elimination process which is a symbolic rewriting process of a certain type that transforms formal systems of equations in groups or semigroups. This process can be viewed as a non-commutative analog of the classical elimination process in algebraic geometry, hence the name. The original version of the process was introduced by Makanin in [51. He showed that the process gives a decision algorithm to verify whether a given system is consistent or not (decidability of Diophantine problem over free groups) by estimating the length of the minimal solution (if it exists). In $6 \mathbf{6 2}$ Razborov developed the process much further, so that the Makanin-Razborov's process produces all solutions of a system of equations in a free group. We refined Razborov's version of the 
process in [36 and 40 to show that up to the rational equivalence irreducible algebraic sets over $F$ can be characterized by non-degenerate triangular quasi-quadratic systems, or NTQ systems (see Section 2.13), thus introducing NTQ groups which play an important role in model theory of free groups. Here the "non-degenerate" part corresponds to the extension theorems in the classical theory of elimination for polynomials. In fact, our rewriting process from [36] or [40] by no means is unique, every time it can be easily adjusted to some particular needs. However, there exist several fundamental common features that unify all variations of our's and Razborov's processes. It is worthwhile to mention here two of them. First, only three precisely defined infinite branches (subprocesses) can occur in the process: linear case (Cases 7-10), quadratic case (Cases 11-12), and general JSJ case (Cases 13-15) which includes periodic structures and abelian vertex groups (Case 2). Secondly, groups of automorphisms of the coordinate groups are used in encoding the infinite branches of the process. In what follows we refer to processes of this type as to elimination processes. Observe, that the original Makanin's process lacks some of these features (for example, there are no infinite branches corresponding to the periodic structures).

Notice that the principal ideas from the Makanin's process (elementary and entire transformations, complexity, etc.) were used in $\mathbf{8}$ and in $\mathbf{2 3}$ to prove the classification theorem for finitely generated groups acting freely on $\mathbb{R}$-trees and to describe stable actions on $\mathbb{R}$-trees, via so-called Rip's machine. Later, these results played a key part in the proof of existence of JSJ decompositions of finitely presented groups with a single end 63, 67.

In this paper we show that a modification of the elimination process from $\mathbf{3 6}$ can be used directly to construct effectively JSJ decompositions of finitely generated fully residually free groups, thus avoiding algorithmically difficult detour into the limit actions. Theorem [7.1 establishes a correspondence between infinite branches of an elimination process and splittings of the coordinate group of the system under consideration: the linear case corresponds to thin (or Levitt) type of a subcomplex in 8 and 23, the quadratic case corresponds to the surface type (or interval exchange), periodic structures correspond to toral (or axial) type. Moreover, the automorphism associated with infinite branches of the process are precisely the canonical automorphism of the JSJ decomposition associated with the splittings. Now we are in a position to explain the main idea of the proof.

Let $G$ be a non-abelian finitely generated fully residually free group given as the coordinate group of a finite system of equations $S(A, X)=1$ over $F$. Every solution in $F$ of the system $S(X, A)=1$ gives rise to a homomorphism $\phi: G \rightarrow$ $F$. Composition of $\phi$ with an automorphism $\sigma$ of $G$ provides a new solution of the system $S(X, A)=1$ in $F$. Thus, different types of canonical automorphisms associated with a JSJ decomposition of $G$ induce solutions of the system $S(X, A)=$ 1 of a particular type, that can be recognized in the elimination process as infinite branches of the corresponding type. Conversely, infinite branches in the elimination process for $S(X, A)=1$ provide infinite families of canonical automorphisms of $G$, that determine the corresponding elementary splitting of $G$. This, in combination with Bass-Serre methods and length functions techniques, allows one to reconstruct the JSJ decompositions of $G$. 
0.4. Main results and organization of the paper. In the first section we collect some known results on fully residually free groups, algebraic geometry over groups, and quadratic equations.

In the second section we discuss splittings, JSJ decompositions, and canonical automorphisms. Here we prove an important technical lemma on induced $Q H$ subgroups (Lemma 2.13).

Section 3 contains various results on decidability of algorithmic problems in finitely generated fully residually free groups. Some of them are known (complete proofs are in [36, 46], 53, and ) and some are new.

In Sections 4 -6] we introduce and study an elimination process. Initially, we describe this process as an infinite branching deterministic procedure which starts at a generalized equation $\Omega$ and on each elementary step rewrites a given generalized equation into a finitely many new ones. The procedure gives rise to an infinite directed tree $T(\Omega)$ which is equipped with some extra objects (enriched tree) associated with vertices and edges. Namely, the coordinate groups of the generalized equations (obtained in the process) are associated with vertices, while homomorphisms of the corresponding coordinate groups (related to the elementary rewriting steps) are associated with edges. As was mentioned above infinite branches of this tree are the focal points of these sections. The periodic structures provide the most difficult case, they occupy the whole Section 6 ]

In Sections 7 and 8 we study splittings of the coordinate group and the structure of solutions of a given finite system of equations over free groups. To this end we cut off the infinite branches of the tree $T(\Omega)$ and encode them by groups of canonical automorphisms of the corresponding coordinate groups. This results into two finite enriched trees. In one of them, $T_{\text {dec }}(\Omega)$, every branch describes a splitting of the coordinate group $F_{R(\Omega)}$ of the root equation $\Omega$. In the other, $T_{\text {sol }}(\Omega)$, every vertex $v$ is associated with the coordinate group $F_{R\left(\Omega_{v}\right)}$ of the corresponding system $\Omega_{v}$ and also with a group of the canonical automorphisms $A_{v}$ of this coordinate group, as before edges $u \rightarrow v$ of $T_{\text {sol }}(\Omega)$ are marked by homomorphisms $F_{R\left(\Omega_{u}\right)} \rightarrow F_{R\left(\Omega_{v}\right)}$. We refer to the latter graphs as to Hom-diagrams. The tree $T_{\operatorname{dec}}(\Omega)$ is the main instrument to recognize all splittings essential to build JSJ decompositions of the coordinate group $F_{R(\Omega)}$ (Theorem [7.7). The tree $T_{\text {sol }}(\Omega)$ gives, in particular, a description of all solutions in $F$ of the initial equation (Theorem 8.1).

In Section 9 we prove

THEOREM 9.1. Let $G$ be a finitely generated freely indecomposable [freely indecomposable modulo $F]$ fully residually free group $G$. Then a non-degenerate JSJ $\mathbb{Z}$ - decomposition [modulo $F$ ] of $G$ is a sufficient splitting of $G$ (see the definition in Subsection 2.19).

In the same section we prove Theorem 9.3 about canonical embeddings of finitely generated fully residually free groups into NTQ groups. This result explains how the top quadratic system of equations in an NTQ system assigned to the NTQ group corresponds to a cyclic JSJ decomposition of the group that we embed.

In Section 10 we give an algorithm to find Grushko's decompositions of finitely generated fully residually free groups. Recall, that a free decomposition

$$
G=G_{1} * \cdots * G_{k} * F_{r}
$$

is a Grushko's decomposition of a group $G$ if the factors $G_{1}, \cdots, G_{k}$ are freely indecomposable non-cyclic groups and the factor $F_{r}$ is a free group of rank $r$. It is known 
that such decompositions of $G$ are essentially unique, i.e., any other decomposition of $G$ of this type has the same numbers $k$ and $r$ and the indecomposable non-cyclic factors are conjugated in $G$ to one of the factors $G_{1}, \cdots, G_{k}$. The next result shows that Gruschko's decompositions (even modulo a given finitely generated subgroup) of $G$ are effective.

THEOREM 10.1 There is an algorithm which for every finitely generated fully residually free group $G$ and its finitely generated subgroup $H$ determines whether or not $G$ has a nontrivial free decomposition such that $H$ belongs to one of the factors. Moreover, if $G$ does have such a decomposition, the algorithm finds one (by giving finite generating sets of the factors).

This theorem allows one to reduce algorithmic problems for finitely generated fully residually free groups to the freely indecomposable ones. For such groups we prove the following theorem which is one of the major results of this paper.

THEOREM 0.1. There exists an algorithm to obtain a cyclic [abelian] JSJ decomposition of a freely indecomposable finitely generated fully residually free group. The algorithm constructs a presentation of this group as the fundamental group of a JSJ graph of groups.

Actually, we prove (in Section 13) the following much more general result which implies Theorem 0.1

THEOREM 13.1 There exists an algorithm to obtain a cyclic [abelian] JSJ decomposition for a finitely generated fully residually free group modulo a given finite family of finitely generated subgroups.

Combining Theorems Theorem 10.1 and 13.1 one can find effectively cyclic [abelian] JSJ decompositions of the freely indecomposable non-abelian factors $G_{1}, \cdots, G_{k}$ of the Grushko's decomposition of $G$. This gives a cyclic [abelian] splitting of $G$ which completely describes the structure of $G$ as the fundamental group of a graph of groups.

A variation of Theorem 0.1 first appeared in 39 (in terms of the elimination processes), where it was used in the proof of the second Tarski's conjecture on the decidability of the elementary theory of free non-abelian groups.

Theorem 0.1 has numerous applications. Using Theorem 0.1 we refine in Theorem 11.2 the effective description of a solution set of a system of equations over $F$ from [36. In Section 14 we give a description of solution sets of systems of equations over finitely generated fully residually free groups. To do this we need the notion of a Hom-diagram described in Section 11.

THEOREM 11.2 Let $G$ be a finitely generated group and $F$ a free group. Then:

(1) there exists a complete canonical $\operatorname{Hom}(G, F)$-diagram $\mathcal{C}$.

(2) if $F$ is a fixed subgroup of $G$ then there exists an $F$-complete canonical $\operatorname{Hom}_{F}(G, F)$-diagram $\mathcal{C}$.

Moreover, if the group $G$ is finitely presented then the Hom-diagrams from (1) and (2) can be found effectively.

This theorem implies the following.

THEOREM 11.3 Let $S(X)=1$ be a finite system of equations and $G=(F * F[X]) / n c l(S)$. Then one can effectively construct an $F$-complete canonical $\operatorname{Hom}(G, F)$-diagram $\mathcal{C}$ such that all solutions of $S(X)=1$ in $F$ are exactly all $F$ homomorphisms in $\mathcal{C}$. 
THEOREM 14.1 Let $G$ be a finitely generated group and $N$ an $N T Q$ group. Then:

(1) there exists a complete canonical $\operatorname{Hom}(G, N)$-diagram $\mathcal{C}$.

(2) if $N$ is a fixed subgroup of $G$ then there exists an $N$-complete canonical $\operatorname{Hom}_{N}(G, N)$-diagram $\mathcal{C}$.

Moreover, if the group $G$ is finitely presented then the diagrams from (1) and (2) can be found effectively.

This theorem implies the following result

COROLLARY 14.2 Let $G$ be a finitely generated group and $H$ a finitely generated fully residually free group. Then:

(1) there exists a complete canonical Hom $(G, H)$-diagram.

(2) if $H$ is a fixed subgroup of $G$ then there exists an $H$-complete canonical $\mathrm{Hom}_{H}(G, H)$-diagram.

In Section 12 we construct free regular Lyndon length functions on NTQ groups.

THEOREM 12.6. If $G$ is the coordinate group of a regular quadratic equation, then there exists a free regular Lyndon length function $\ell: G \rightarrow \mathbb{Z}^{2}$, where $\mathbb{Z}^{2}$ is ordered lexicographically.

THEOREM 12.7. If $G$ is the coordinate group of an NTQ system, then there exists a free regular Lyndon length function $\ell: G \rightarrow \mathbb{Z}^{t}$, where $\mathbb{Z}^{t}$ is ordered lexicographically.

In Section 15 we prove some results about monomorphisms into fully residually free groups.

Let $G$ and $H$ be finitely generated fully residually free groups. Consider monomorphisms from $G$ to $H$. One can define an equivalence relation on the set of all such monomorphisms: two monomorphisms $\phi$ and $\psi$ are equivalent if $\psi$ is a composition of $\phi$ and conjugation by an element from $H$.

THEOREM 15.1 Let $G[H]$ be a finitely generated fully residually free group, and let $\mathcal{A}=\left\{A_{1}, \ldots, A_{n}\right\}$ [respectively, $\left.\mathcal{B}=\left\{B_{1}, \ldots, B_{n}\right\}\right]$ be a finite set of nonconjugated maximal abelian subgroups of $G$ [respectively, $H]$ such that the abelian decomposition of $G$ modulo $\mathcal{A}$ is trivial. The number of equivalence classes of monomorphisms from $G$ to $H$ that map subgroups from $\mathcal{A}$ onto conjugates of the corresponding subgroups from $\mathcal{B}$ is finite. A set of representatives of the equivalence classes can be effectively found.

COROLlaRY 15.2 Let $G$ be a finitely generated fully residually free group, and let $\mathcal{A}=\left\{A_{1}, \ldots, A_{n}\right\}$ be a finite set of maximal abelian subgroups of $G$. Denote by $\operatorname{Out}(G ; \mathcal{A})$ the set of those outer automorphisms of $G$ which map each $A_{i} \in \mathcal{A}$ onto a conjugate of it. If $\operatorname{Out}(G ; \mathcal{A})$ is infinite, then $G$ has a non-trivial abelian splitting, where each subgroup in $\mathcal{A}$ is elliptic. There is an algorithm to decide whether $\operatorname{Out}(G ; \mathcal{A})$ is finite or infinite. If Out $(G ; \mathcal{A})$ is infinite, the algorithm finds the splitting. If $\operatorname{Out}(G ; \mathcal{A})$ is finite, the algorithm finds all its elements.

This can be compared to the following Paulin's result [59. If $G$ is a hyperbolic group that has infinite $\operatorname{Out}(G)$, then $G$ admits a non-trivial small action on a real tree. In this case $G$ splits over an elementary group $\mathbf{8}$.

\section{Preliminaries}

1.1. Free monoids and free groups. Let $A=\left\{a_{1}, \ldots, a_{m}\right\}$ be a set. By $F_{\text {mon }}(A)$ we denote the free monoid generated by $A$ which is defined as the set of 
all words (including the empty word 1 ) over the alphabet $A$ with concatenation as multiplication. For a word $w=b_{1} \ldots b_{n}$, where $b_{i} \in A$, by $|w|$ or $d(w)$ we denote the length $n$ of $w$.

To each $a \in A$ we associate a symbol $a^{-1}$. Put $A^{-1}=\left\{a^{-1} \mid a \in A\right\}$, and suppose that $A \cap A^{-1}=\emptyset$. We assume that $a^{1}=a,\left(a^{-1}\right)^{-1}=a$ and $A^{1}=A$. Denote $A^{ \pm 1}=A \cup A^{-1}$. If $w=b_{1}^{\varepsilon_{1}} \ldots b_{n}^{\varepsilon_{n}} \in F_{\text {mon }}\left(A^{ \pm 1}\right)$, where $\left(\varepsilon_{i} \in\{1,-1\}\right)$, then we put $w^{-1}=b_{1}^{-\varepsilon_{1}} \ldots b_{n}^{-\varepsilon_{n}}$; we see that $w^{-1} \in M\left(A^{ \pm 1}\right)$ and say that $w^{-1}$ is an inverse of $w$. Furthermore, we put $1^{-1}=1$.

A word $w \in F_{m o n}\left(A^{ \pm 1}\right)$ is called reduced if it does not contain subwords $b b^{-1}$ for $b \in A^{ \pm 1}$. If $w=w_{1} b b^{-1} w_{2}, w \in F_{\text {mon }}\left(A^{ \pm 1}\right)$ then we say that $w_{1} w_{2}$ is obtained from $w$ by an elementary reduction $b b^{-1} \rightarrow 1$. A reduction process for $w$ consists of finitely many reductions which bring $w$ to a reduced word $\bar{w}$. This $\bar{w}$ does not depend on a particular reduction process and is called the reduced form of $w$.

Consider a congruence relation on $F_{m o n}\left(A^{ \pm 1}\right)$, defined the following way: two words are congruent if a reduction process brings them to the same reduced word. The set of congruence classes with respect to this relation forms a free group $F(A)$ with basis $A$. If not said otherwise, we assume that $F(A)$ is given as the set of all reduced words in $A^{ \pm 1}$. Multiplication in $F(A)$ of two words $u, w$ is given by the reduced form of their concatenation, i.e., $u \cdot v=\overline{u v}$. A word $w \in F_{m o n}\left(A^{ \pm 1}\right)$ determines the element $\bar{w} \in F(A)$, in this event we sometimes say that $w$ is an element of $F(A)$ (even though $w$ may not be reduced).

Words $u, w \in F_{\text {mon }}\left(A^{ \pm 1}\right)$ are graphically equal if they are equal in the monoid $F_{m o n}\left(A^{ \pm 1}\right)$ (for example, $a_{1} a_{2} a_{2}^{-1}$ is not graphically equal to $a_{1}$ ).

Let $X=\left\{x_{1}, \ldots, x_{n}\right\}$ be a finite set of elements disjoint with $A$. Let $w(X)=$ $w\left(x_{1}, \ldots, x_{n}\right)$ be a word in the alphabet $(X \cup A)^{ \pm 1}$ and $U=\left(u_{1}(A), \ldots, u_{n}(A)\right)$ be a tuple of words in the alphabet $A^{ \pm 1}$. By $w(U)$ we denote the word which is obtained from $w$ by replacing each $x_{i}$ by $u_{i}$. Similarly, if $W=\left(w_{1}(X), \ldots, w_{m}(X)\right)$ is an $m$ tuple of words in variables $X$ then by $W(U)$ we denote the tuple $\left(w_{1}(U), \ldots, w_{m}(U)\right)$. For any set $S$ we denote by $S^{n}$ the set of all $n$-tuples of elements from $S$. Every word $w(X)$ gives rise to a map $p_{w}:\left(F_{m o n}\left(A^{ \pm 1}\right)\right)^{n} \rightarrow F_{m o n}\left(A^{ \pm 1}\right)$ defined by $p_{w}(U)=w(U)$ for $U \in F_{m o n}\left(A^{ \pm 1}\right)^{n}$. We call $p_{w}$ the word map defined by $w(X)$. If $W(X)=\left(w_{1}(X), \ldots, w_{m}(X)\right)$ is an $m$-tuple of words in variables $X$ then we define a word map $P_{W}:\left(F_{\text {mon }}\left(A^{ \pm 1}\right)\right)^{n} \rightarrow F_{m o n}\left(A^{ \pm 1}\right)^{m}$ by the rule $P_{W}(U)=W(U)$.

1.2. $G$-groups. For the purpose of algebraic geometry over a given fixed group $G$, one has to consider the category of $G$-groups, i.e., groups which contain the group $G$ as a distinguished subgroup. If $H$ and $K$ are $G$-groups then a homomorphism $\phi: H \rightarrow K$ is a $G$ - homomorphism if $g^{\phi}=g$ for every $g \in G$, in this event we write $\phi: H \rightarrow_{G} K$. In this category morphisms are $G$-homomorphisms; subgroups are $G$-subgroups, etc. By $\operatorname{Hom}_{G}(H, K)$ we denote the set of all $G$-homomorphisms from $H$ into $K$. It is not hard to see that the free product $G * F(X)$ is a free object in the category of $G$-groups. This group is called a free $G$-group with basis $X$, and we denote it by $G[X]$. A $G$-group $H$ is termed finitely generated $G$-group if there exists a finite subset $A \subset H$ such that the set $G \cup A$ generates $H$. We refer to [4] for a general discussion on $G$-groups.

To deal with cancellation in the group $G[X]$ we need the following notation. Let $u=u_{1} \ldots u_{n} \in G[X]=G * F(X)$. We say that $u$ is reduced (as written) if $u_{i} \neq 1, u_{i}$ and $u_{i+1}$ are in different factors of the free product, and if $u_{i} \in F(X)$ then it is reduced in the free group $F(X)$. By $\operatorname{red}(u)$ we denote the reduced form 
of $u$. If $\operatorname{red}(u)=u_{1} \ldots u_{n} \in G[X]$, then we define $|u|=n$, so $|u|$ is the syllable length of $u$ in the free product $G[X]$. For reduced $u, v \in G[X]$, we write $u \circ v$ if the product $u v$ is reduced as written. If $u=u_{1} \ldots u_{n}$ is reduced and $u_{1}, u_{n}$ are in different factors, then we say that $u$ is cyclically reduced.

If $u=r \circ s, v=s^{-1} \circ t$, and $r t=r \circ t$ then we say that the word $s$ cancels out in reducing $u v$, or, simply, $s$ cancels out in $u v$. Therefore $s$ corresponds to the maximal cancellation in $u v$.

1.3. Elements of algebraic geometry over groups. Here we introduce some basic notions of algebraic geometry over groups. We refer to [4 and [34 for details.

Let $G$ be a group generated by a finite set $A, F(X)$ be a free group with basis $X=\left\{x_{1}, x_{2}, \ldots x_{n}\right\}, G[X]=G * F(X)$ be a free product of $G$ and $F(X)$. If $S \subset G[X]$ then the expression $S=1$ is called a system of equations over $G$. As an element of the free product, the left side of every equation in $S=1$ can be written as a product of some elements from $X \cup X^{-1}$ (which are called variables) and some elements from $A$ (constants). To emphasize this we sometimes write $S(X, A)=1$.

A solution of the system $S(X)=1$ over a group $G$ is a tuple of elements $g_{1}, \ldots, g_{n} \in G$ such that after replacement of each $x_{i}$ by $g_{i}$ the left hand side of every equation in $S=1$ turns into the trivial element of $G$. Equivalently, a solution of the system $S=1$ over $G$ can be described as a $G$-homomorphism $\phi: G[X] \longrightarrow G$ such that $\phi(S)=1$. Denote by $\operatorname{ncl}(S)$ the normal closure of $S$ in $G[X]$, and by $G_{S}$ the quotient group $G[X] / n c l(S)$. Then every solution of $S(X)=1$ in $G$ gives rise to a $G$-homomorphism $G_{S} \rightarrow G$, and vice versa. By $V_{G}(S)$ we denote the set of all solutions in $G$ of the system $S=1$, it is called the algebraic set defined by $S$. This algebraic set $V_{G}(S)$ uniquely corresponds to the normal subgroup

$$
R(S)=\left\{T(x) \in G[X] \mid \forall A \in G^{n}(S(A)=1 \rightarrow T(A)=1\}\right.
$$

of the group $G[X]$. Notice that if $V_{G}(S)=\emptyset$, then $R(S)=G[X]$. The subgroup $R(S)$ contains $S$, and it is called the radical of $S$. The quotient group

$$
G_{R(S)}=G[X] / R(S)
$$

is the coordinate group of the algebraic set $V(S)$. Again, every solution of $S(X)=1$ in $G$ can be described as a $G$-homomorphism $G_{R(S)} \rightarrow G$.

We recall from [55] that a group $G$ is called a CSA group if every maximal abelian subgroup $M$ of $G$ is malnormal, i.e., $M^{g} \cap M=1$ for any $g \in G \backslash M$. The class of CSA-groups is quite substantial. It includes all abelian groups, all torsion-free hyperbolic groups [55, all groups acting freely on $\Lambda$-trees [3], and many one-relator groups 24 .

We define a Zariski topology on $G^{n}$ by taking algebraic sets in $G^{n}$ as a sub-basis for the closed sets of this topology. If $G$ is a non-abelian CSA group, in particular, a non-abelian fully residually free group (see definition in Subsection 1.4), then the union of two algebraic sets is again algebraic. Therefore the closed sets in the Zariski topology over $G$ are precisely the algebraic sets.

A $G$-group $H$ is called equationally Noetherian if every system $S(X)=1$ with coefficients from $G$ is equivalent over $G$ to a finite subsystem $S_{0}=1$, where $S_{0} \subset S$, i.e., $V_{G}(S)=V_{G}\left(S_{0}\right)$. If $G$ is $G$-equationally Noetherian, then we say that $G$ is equationally Noetherian. It is known that linear groups (in particular, fully residually free groups) are equationally Noetherian (see $[\mathbf{2 7}, \mathbf{7}, \mathbf{4}]$ ). If $G$ is equationally 
Noetherian then the Zariski topology over $G^{n}$ is Noetherian for every $n$, i.e., every proper descending chain of closed sets in $G^{n}$ is finite. This implies that every algebraic set $V$ in $G^{n}$ is a finite union of irreducible subsets (they are called $i r$ reducible components of $V$ ), and such decomposition of $V$ is unique. Recall that a closed subset $V$ is irreducible if it is not a union of two proper closed (in the induced topology) subsets. Denote by $F_{\omega R(S)}$ a coordinate group of an irreducible subvariety of $V(S)$.

1.4. Discrimination and big powers. Let $H$ and $K$ be $G$-groups. We say that a family of $G$ - homomorphisms $\Phi \subset \operatorname{Hom}_{G}(H, K)$ separates (discriminates) $H$ into $K$ if for every non-trivial element $h \in H$ (every finite set of non-trivial elements $\left.H_{0} \subset H\right)$ there exists $\phi \in \Phi$ such that $h^{\phi} \neq 1\left(h^{\phi} \neq 1\right.$ for every $\left.h \in H_{0}\right)$. In this case we say that $H$ is $G$-separated ( $G$-discriminated) by $K$. Sometimes we do not mention $G$ and simply say, $H$ is separated (discriminated) by $K$. In the event when $K$ is a free group we say that $H$ is freely separated (freely discriminated).

Below we describe a method of discrimination which is called a big powers method. We refer to $\mathbf{5 5}$ and $\mathbf{5 4}$ for details about BP-groups.

Let $G$ be a group. We say that a tuple $u=\left(u_{1}, \ldots, u_{k}\right) \in G^{k}$ has commutation if $\left[u_{i}, u_{i+1}\right]=1$ for some $i=1, \ldots k-1$. Otherwise we call $u$ commutation-free.

Definition 1.1. A group $G$ satisfies the big powers condition (BP) if for any commutation-free tuple $u=\left(u_{1}, \ldots, u_{k}\right)$ of elements from $G$ there exists an integer $n(u)$ (a boundary of separation for $u$ ) such that

$$
u_{1}^{\alpha_{1}} \ldots u_{k}^{\alpha_{k}} \neq 1
$$

for any integers $\alpha_{1}, \ldots, \alpha_{k} \geq n(u)$. Such groups are called BP-groups.

The following provides a host of examples of BP-groups. Obviously, a subgroup of a BP-group is a BP-group; a group discriminated by a BP-group is a $\mathrm{BP}$ - group 55; every torsion-free hyperbolic group is a BP-group [58. It follows that every freely discriminated group is a BP-group.

Let $G$ be a non-abelian CSA group and $u \in G$ be a non-proper power. The following HNN-extension

$$
G(u, t)=\left\langle G, t \mid g^{t}=g\left(g \in C_{G}(u)\right)\right\rangle
$$

is called a free extension of the centralizer $C_{G}(u)$ by a letter $t$. It is not hard to see that for any integer $k$ the map $t \rightarrow u^{k}$ extends uniquely to a $G$-homomorphism $\xi_{k}: G(u, t) \rightarrow G$.

The result below is the essence of the big powers method of discrimination.

TheOrem 1.2. [55 Let $G$ be a non-abelian CSA BP-group and $u \in G$ a nonproper power. If $G(u, t)$ is a free extension of the centralizer of $u$ by $t$, then the family of $G$-homomorphisms $\left\{\xi_{k} \mid k\right.$ is an integer $\}$ discriminates $G(u, v)$ into $G$. More precisely, for every $w \in G(u, t)$ there exists an integer $N_{w}$ such that for every $k \geq N_{w} w^{\xi_{k}} \neq 1$.

If $G$ is a non-abelian CSA BP-group and $X$ is a finite set, then the group $G[X]$ is $G$-embeddable into $G(u, t)$ for any non-proper power $u \in G$. It follows from the theorem above that $G[X]$ is $G$-discriminated by $G$.

Unions of chains of extensions of centralizers play an important part in this paper. Let $G$ be a non-abelian CSA BP-group and

$$
G=G_{0}<G_{1}<\ldots<G_{n}
$$


be a chain of extensions of centralizers $G_{i+1}=G_{i}\left(u_{i}, t_{i}\right)$. Then every $n$-tuple of integers $p=\left(p_{1}, \ldots, p_{n}\right)$ gives rise to a $G$-homomorphism $\xi_{p}: G_{n} \rightarrow G$ which is composition of homomorphisms $\xi_{p_{i}}: G_{i} \rightarrow G_{i-1}$ described above.

A set $P$ of $n$-tuples of integers is called unbounded if for every integer $d$ there exists a tuple $p=\left(p_{1}, \ldots, p_{n}\right) \in P$ with $p_{i} \geq d$ for each $i$. The following result is a consequence of the theorem above.

Corollary 1.3. Let $G_{n}$ be as above. Then for every unbounded set of tuples $P$ the set of $G$-homomorphisms $\Xi_{P}=\left\{\xi_{p} \mid p \in P\right\}$ G-discriminates $G_{n}$ into $G$.

Similar results hold for infinite chains of extensions of centralizers (see [55]) and [5]). For example, Lyndon's free $Z[x]$-group $F^{Z[x]}$ can be realized as union of a countable chain of extensions of centralizers which starts with the free group $F$ (see [55]), hence there exists a family of $F$-homomorphisms which discriminates $F^{Z[x]}$ into $F$.

1.5. Fully residually free groups. Denote by $\mathcal{F}$ the class of all finitely generated fully residually free groups. In this section we describe some properties of groups from $\mathcal{F}$, which are crucial for our considerations.

It is not hard to see that every freely discriminated group is a torsion-free CSA group 4 .

Notice that every CSA group is commutation transitive [55]. A group $G$ is called commutation transitive if commutation is transitive on the set of all nontrivial elements of $G$, i.e., if $a, b, c \in G \backslash\{1\}$ and $[a, b]=1,[b, c]=1$, then $[a, c]=1$. Clearly, commutation transitive groups are precisely the groups in which centralizers of non-trivial elements are commutative.

TheOREM 1.4. 66 Let $F$ be a free non-abelian group. Then a finitely generated $F$-group $G$ is freely $F$-discriminated by $F$ if and only if $G$ is $F$-universally equivalent to $F$ (i.e., $G$ and $F$ satisfy precisely the same universal sentences in the language $\left.L_{A}\right)$.

TheOREm 1.5. [4, 34] Let $F$ be a free non-abelian group. Then a finitely generated $F$-group $G$ is the coordinate group of a non-empty irreducible algebraic set over $F$ if and only if $G$ is freely $F$-discriminated by $F$.

THEOREM 1.6. 35 Let $F$ be a non-abelian free group. Then a finitely generated $F$-group is the coordinate group $F_{R(S)}$ of an irreducible non-empty algebraic set $V(S)$ over $F$ if and only if $G$ is $F$-embeddable into the free Lyndon's $Z[t]$-group $F^{Z[t]}$.

Here we state one corollary of the results mentioned above.

TheOREM 1.7. 36 Every group $G \in \mathcal{F}$ can be obtained from free groups by finitely many operations of the following type:

(1) free products;

(2) free products with amalgamation along cyclic subgroups with at least one of them being maximal;

(3) separated HNN extensions along cyclic subgroups with at least one of them being maximal; 
(4) free extensions of centralizers (and for each element its centralizer extends at most once).

Corollary 1.8. 36, 69 Every finitely generated fully residually free group is finitely presented.

1.6. Quadratic equations over freely discriminated groups. In this section we collect some known results about quadratic equations over fully residually free groups, which will be in use throughout this paper.

Let $S \subset G[X]$. Denote by $\operatorname{var}(S)$ the set of variables that occur in $S$.

Definition 1.9. A set $S \subset G[X]$ is called quadratic if every variable from $\operatorname{var}(S)$ occurs in $S$ not more then twice. The set $S$ is strictly quadratic if every letter from $\operatorname{var}(S)$ occurs in $S$ exactly twice.

A system $S=1$ over $G$ is quadratic [strictly quadratic] if the corresponding set $S$ is quadratic [strictly quadratic].

Definition 1.10. A standard quadratic equation over the group $G$ is an equation of the one of the following forms (below $d, c_{i}$ are nontrivial elements from $G$ ):

$$
\begin{gathered}
\prod_{i=1}^{n}\left[x_{i}, y_{i}\right]=1, \quad n>0 ; \\
\prod_{i=1}^{n}\left[x_{i}, y_{i}\right] \prod_{i=1}^{m} z_{i}^{-1} c_{i} z_{i} d=1, \quad n, m \geq 0, m+n \geq 1 ; \\
\prod_{i=1}^{n} x_{i}^{2}=1, \quad n>0 ; \\
\prod_{i=1}^{n} x_{i}^{2} \prod_{i=1}^{m} z_{i}^{-1} c_{i} z_{i} d=1, \quad n, m \geq 0, n+m \geq 1 .
\end{gathered}
$$

Equations (11), (2) are called orientable of genus $n$, equations (3), (4) are called non-orientable of genus $n$.

Lemma 1.11. Let $W$ be a strictly quadratic word over a group $G$. Then there is a $G$-automorphism $f \in$ Aut $_{G}(G[X])$ such that $W^{f}$ is a standard quadratic word over $G$.

Proof. See 49].

Definition 1.12. Strictly quadratic words of the type $[x, y], x^{2}, z^{-1} c z$, where $c \in G$, are called atomic quadratic words or simply atoms.

By definition a standard quadratic equation $S=1$ over $G$ has the form

$$
r_{1} r_{2} \ldots r_{k} d=1 \text {, }
$$

where $r_{i}$ are atoms, $d \in G$. This number $k$ is called the atomic rank of this equation, we denote it by $r(S)$. The size of $S=1$ is a pair size $(S)=(g(S), r(S))$, where $g(S)$ is the genus of $S=1$. We compare sizes lexicographically from the left.

In Section 1.3 we defined the notion of the coordinate group $G_{R(S)}$. Every solution of the system $S=1$ is a homomorphism $\phi: G_{R(S)} \rightarrow G$. 
Definition 1.13. Let $S=1$ be a standard quadratic equation written in the atomic form $r_{1} r_{2} \ldots r_{k} d=1$ with $k \geq 2$. A solution $\phi: G_{R(S)} \rightarrow G$ of $S=1$ is called:

1) degenerate, if $r_{i}^{\phi}=1$ for some $i$, and non-degenerate otherwise;

2) commutative, if $\left[r_{i}^{\phi}, r_{i+1}^{\phi}\right]=1$ for all $i=1, \ldots, k-1$, and non-commutative otherwise;

3) in a general position, if $\left[r_{i}^{\phi}, r_{i+1}^{\phi}\right] \neq 1$ for all $i=1, \ldots, k-1$,.

Observe that if a standard quadratic equation $S(X)=1$ has a degenerate noncommutative solution then it has a non-degenerate non-commutative solution see 35).

Theorem 1.14 ([35]). Let $G$ be a freely discriminated group and $S=1$ a standard quadratic equation over $G$ which has a solution in $G$. In the following cases $S=1$ always has a solution in $G$ in a general position:

(1) $S=1$ is of the form (1), $n>2$;

(2) $S=1$ is of the form (2), $n>0, n+m>1$;

(3) $S=1$ is of the form (3), $n>3$;

(4) $S=1$ is of the form (4), $n>2$;

(5) $r(S) \geq 2$ and $S=1$ has a non-commutative solution.

The following theorem describes the radical $R(S)$ of a standard quadratic equation $S=1$ which has at least one solution in a freely discriminated group $G$.

TheOREM 1.15 ([35). Let $G$ be a freely discriminated group and let $S=1$ be a standard quadratic equation over $G$ which has a solution in $G$. Then

(1) If $S=[x, y] d$ or $S=\left[x_{1}, y_{1}\right]\left[x_{2}, y_{2}\right]$, then $R(S)=n c l(S)$;

(2) If $S=x^{2} d$, then $R(S)=n c l(x b)$ where $b^{2}=d$;

(3) If $S=c^{z} d$, then $R(S)=n c l\left(\left[z b^{-1}, c\right]\right)$ where $d^{-1}=c^{b}$;

(4) If $S=x_{1}^{2} x_{2}^{2}$, then $R(S)=n c l\left(\left[x_{1}, x_{2}\right]\right)$;

(5) If $S=x_{1}^{2} x_{2}^{2} x_{3}^{2}$, then $R(S)=n c l\left(\left[x_{1}, x_{2}\right],\left[x_{1}, x_{3}\right],\left[x_{2}, x_{3}\right]\right)$;

(6) If $r(S) \geq 2$ and $S=1$ has a non-commutative solution, then $R(S)=$ $n \operatorname{ncl}(S)$;

(7) If $S=1$ is of the type (4) and all solutions of $S=1$ are commutative, then $R(S)$ is the normal closure of the following system:

$$
\begin{gathered}
\left\{x_{1} \ldots x_{n}=s_{1} \ldots s_{n},\left[x_{k}, x_{l}\right]=1,\left[a_{i}^{-1} z_{i}, x_{k}\right]=1,\left[x_{k}, C\right]=1,\left[a_{i}^{-1} z_{i}, C\right]=1,\right. \\
\left.\left[a_{i}^{-1} z_{i}, a_{j}^{-1} z_{j}\right]=1 \quad(k, l=1, \ldots, n ; i, j=1, \ldots, m)\right\} \\
\text { where } x_{k} \rightarrow s_{k}, z_{i} \rightarrow a_{i} \text { is a solution of } S=1 \text { and } \\
C=C_{G}\left(c_{1}^{a_{1}}, \ldots, c_{m}^{a_{m}}, s_{1}, \ldots, s_{n}\right)
\end{gathered}
$$

is the corresponding centralizer. The group $G_{R(S)}$ is an extension of the centralizer $C$.

Put

$$
\kappa(S)=|X|+\varepsilon(S)
$$

where $\varepsilon(S)=1$ if the coefficient $d$ occurs in $S$, and $\varepsilon(S)=0$ otherwise.

Definition 1.16. A standard quadratic equation $S(X)=1$ is regular if $\kappa(S) \geq$ 4 and there is a non-commutative solution of $S(X)=1$ in $G$, or it is an equation of the type $[x, y] d=1$. 
Notice, that if $S(X)=1$ has a solution in $G, \kappa(S) \geq 4$, and $n>0$ in the orientable case ( $n>2$ in the non-orientable case), then the equation $S=1$ has a non-commutative solution, hence regular.

COROLlaRY 1.17. (1) Every consistent orientable quadratic equation $S(X)=$ 1 of positive genus is regular, unless it is the equation $[x, y]=1$;

(2) Every consistent non-orientable equation of positive genus is regular, unless it is an equation of the type $x^{2} c^{z}=a^{2} c, x_{1}^{2} x_{2}^{2}=a_{1}^{2} a_{2}^{2}, x_{1}^{2} x_{2}^{2} x_{3}^{2}=1$, or $S(X)=1$ can be transformed to the form $\left[\bar{z}_{i}, \bar{z}_{j}\right]=\left[\bar{z}_{i}, a\right]=1, i, j=$ $1, \ldots, m$ by changing variables.

(3) Every standard quadratic equation $S(X)=1$ of genus 0 is regular unless either it is an equation of the type $c_{1}^{z_{1}}=d, c_{1}^{z_{1}} c_{2}^{z_{2}}=c_{1} c_{2}$, or $S(X)=1$ can be transformed to the form $\left[\bar{z}_{i}, \bar{z}_{j}\right]=\left[\bar{z}_{i}, a\right]=1, i, j=1, \ldots, m$ by changing variables.

\section{Splittings}

2.1. Graphs. A directed graph $X$ consists of a set of vertices $V(X)$ and a set of edges $E(X)$ together with two functions $\sigma: E(X) \rightarrow V(X), \tau: E(X) \rightarrow V(X)$. For an edge $e \in E(X)$ the vertices $\sigma(e)$ and $\tau(e)$ are called the origin and the terminus of $e$. A non-oriented graph is a directed graph $X$ with involution $-: E(X) \rightarrow E(X)$ which satisfies the following conditions:

$$
\overline{\bar{e}}=e, e \neq \bar{e}, \sigma(\bar{e})=\tau(e) .
$$

We refer to a pair $\{e, \bar{e}\}$ as a non-oriented edge.

A path $p$ in a graph $X$ is a sequence if edges $e_{1}, \ldots, e_{n}$ such that $\tau\left(e_{i}\right)=$ $\sigma\left(e_{i+1}\right), i \in\{1, \ldots, n-1\}$. Put $\sigma(p)=\sigma\left(e_{1}\right), \tau(p)=\tau\left(e_{n}\right)$. A path $p=e_{1} \ldots e_{n}$ is reduced if $e_{i+1} \neq \bar{e}_{i}$ for each $i$. A path $p$ is closed (or a loop) if $\sigma(p)=\tau(p)$.

2.2. Graphs of groups. A graph of groups $\Gamma=\mathcal{G}(X)$ is defined by the following data:

1) a connected graph $X$;

2) a function $\mathcal{G}$ which for every vertex $v \in V(X)$ assigns a group $G_{v}$, and for each edge $e \in E(X)$ assigns a group $G_{e}$ such that $G_{\bar{e}}=G_{e}, \sigma\left(G_{e}\right)=$ $\tau\left(G_{\bar{e}}\right)$;

3) For each edge $e \in E(X)$ there are monomorphisms $\sigma: G_{e} \rightarrow G_{e \sigma}$ and $\tau: G_{e} \rightarrow G_{e \tau}$.

Let $\mathcal{G}(X)$ be a graph of groups and $T$ a maximal subtree of $X$. The fundamental group $\pi(\mathcal{G}(X), T)$ of the graph of groups $\mathcal{G}(X)$ with respect to the tree $T$ is defined by generators and relations as follows: $\pi(\mathcal{G}(X), T)=$

$$
\left\langle\left(*_{v \in V(X)} G_{v}\right), t_{e}(e \in E(X)) \mid t_{e}=1(e \in T), t_{e}^{-1} g t_{e}=g^{\tau}\left(g \in G_{e}\right), t_{e} t_{\bar{e}}=1\right\rangle .
$$

It is known that $\pi(\mathcal{G}(X), T)$ is independent (up to isomorphism) of $T$. Therefore, we will omit sometimes the tree $T$ from the notations and write simply $\pi(\mathcal{G}(X)$ ).

If some presentation is fixed, the non-trivial generators $t_{e}$ will be called stable letters. The group $\pi(\mathcal{G}(X))$ can be obtained from the vertex groups by a tree product with amalgamation and then by HNN-extensions. The following lemma shows that subgroups of $\pi(\mathcal{G}(X))$ are again fundamental groups of some special graphs of groups related to $\mathcal{G}(X)$. 
Lemma 2.1. 16 Let $\mathcal{G}(X)$ be a graph of groups, and let $H \leq \pi(\mathcal{G}(X))$. Then $H=\pi(\mathcal{G}(Y))$ where the vertex groups of $\mathcal{G}(Y))$ are $H \cap g G_{v} g^{-1}$ for all vertices $v \in X$, and $g$ runs over a suitable set of $\left(H, G_{v}\right)$ double coset representatives, and the edge groups are $H \cap g G_{e} g^{-1}$ for all edges $e \in X$, where $g$ runs over a suitable set of $\left(H, G_{e}\right)$ double coset representatives.

2.3. Definitions and elementary properties of splittings. Let $\pi(\mathcal{G}(X) ; T)$ be the fundamental group of graph of groups $\mathcal{G}(X)$ with respect to a maximal subtree $T$. Let $\phi: G \rightarrow \pi(\mathcal{G}(X) ; T)$ be an isomorphism of groups. In this event the triple $D=(G,(\mathcal{G}(X) ; T), \phi)$ is called a splitting of $G$. A splitting $D$ is a $\mathbb{Z}$-splitting [abelian splitting] if every edge group is infinite cyclic [abelian]. Splittings of the type $G=A *_{C} B$ or $G=A *_{C}$, are called elementary $\mathbb{Z}$-splittings. An elementary abelian splitting $D$ is called essential if the images of the edge group under the boundary monomorphisms do not have finite index in the corresponding vertex groups. A splitting is reduced if all vertex groups of valency one and two properly contain the images of groups of adjacent edges. An abelian splitting is called non-degenerate if its graph of groups is reduced and has an edge. A splitting is non-trivial if its graph of groups has an edge.

Recall, that a splitting of a group $G$ is called 2-acylindrical if for every nontrivial element $g \in G$, the fixed set of $g$ when acting on the Bass-Serre tree corresponding to the splitting has diameter at most 2. A splitting of a group is a star of groups, if its underlying graph is a tree $T$ which has diameter 2 .

2.4. Elementary transformations of graphs of groups and splittings. A conjugation of a splitting of $G$ is a conjugation of $\pi(\mathcal{G}(X) ; T)$.

A sliding is a modification of a graph of groups according to the relation

$$
\left(A_{1} *_{C_{1}} A_{2}\right) *_{C_{2}} A_{3} \cong\left(A_{1} *_{C_{1}} A_{3}\right) *_{C_{2}} A_{2}
$$

in the case when $C_{1} \leq C_{2}$. More precisely, suppose a graph of groups $\Gamma=\mathcal{G}(X)$ contains vertices $v_{1}, v_{2}, v_{3}$ with vertex groups $A_{1}, A_{2}, A_{3}$ respectively, and edges $e_{1}=\left(v_{1}, v_{2}\right), e_{2}=\left(v_{2}, v_{3}\right)$ with edge groups $C_{1}$ and $C_{2}$ and $\tau_{e_{1}}\left(C_{1}\right) \leq \sigma_{e_{2}}\left(C_{2}\right)$. We replace $e_{1}$ by the edge $\bar{e}_{1}=\left(v_{1}, v_{3}\right)$ with edge group $C_{1}$ and $\sigma_{\bar{e}_{1}}\left(C_{1}\right)=$ $\sigma_{e_{1}}\left(C_{1}\right), \tau_{\bar{e}_{1}}\left(C_{1}\right)=\tau_{e_{2}} \sigma_{e_{2}}^{-1} \tau_{e_{1}}\left(C_{1}\right)$. Notice that two of the vertices may coincide.

If $\Gamma$ is a graph of groups, and $G_{e}$ is an edge group in $\Gamma$ such that $\sigma\left(G_{e}\right)=$ $G_{e \sigma}$, then conjugation of the boundary monomorphism $\sigma$ is a replacement of the monomorphism $\sigma$ by $\sigma_{h}$ such that $\sigma_{h}(g)=h^{-1} \sigma(g) h$ for some $h \in G_{e \sigma}$ and any $g \in G_{e}$.

A splitting $G=A *_{C_{1}} B_{1}$ is obtained by folding from the splitting $G=A *_{C} B$ if $C$ is a proper subgroup of $C_{1}$ and $B_{1}=C_{1} *_{C} B$. An unfolding is the inverse operation to folding. A splitting is unfolded if one cannot apply an unfolding to it.

Lemma 2.2. Elementary transformations preserve the fundamental groups of graphs of groups up to isomorphism.

Proof. The statement is obvious for a conjugation. Suppose the graph of groups $\Gamma_{1}=\mathcal{G}_{1}\left(X_{1}\right)$ is obtained from $\Gamma=\mathcal{G}(X)$ by an elementary transformation. Different choice of $T$ in the graph $X$ defines an isomorphism of the fundamental group $\pi(\mathcal{G}(X), T)$. Therefore it is enough to prove the isomorphism of the fundamental groups for a suitable choice of $T$ in $X$ and $T_{1}$ in $X_{1}$. For sliding we consider only the case when vertices $v_{1}, v_{2}, v_{3}$ are different. In this case we choose the tree $T$ 
such a way that edges $e_{1}$ and $e_{2}$ belong to $T$, and obtain $T_{1}$ by the sliding defined above from $T$. Then the isomorphism becomes obvious.

Suppose $\Gamma_{1}=\mathcal{G}_{1}(X)$ is obtained from $\Gamma$ by the conjugation of the boundary monomorphism $\sigma$. Consider first the case when it is possible to choose $T$ such a way that $e \notin T$. In this case $G=\pi(\mathcal{G}(X) ; T)$ has a stable letter $t$ corresponding to $e$ and a relation $t^{-1} \sigma(g) t=\tau(g)$ for any $g \in G_{e}$. Let $t_{h}$ be a stable letter in the presentation $\pi\left(\mathcal{G}_{1}(X) ; T\right)$ which has the corresponding relation $t_{h}^{-1} \sigma_{h}(g) t_{h}=\tau(g)$. These two presentations define the same group, because replacing the generator $t_{h}$ in the second presentation by $h^{-1} t$, we obtain the first presentation. Now consider the case when it is not possible to choose $T$ such a way that $e \notin T$. Then removing $e$ from $X$ we obtain two connected components $X_{1}$ and $X_{2}$. Let $G_{e \sigma} \in X_{1}, G_{1}$ and $G_{2}$ are fundamental groups of the graphs of groups corresponding to $X_{1}$ and $X_{2}$. Then

and

$$
\pi(\mathcal{G}(X) ; T)=G_{1} *_{\sigma\left(G_{e}\right)=\tau\left(G_{e}\right)} G_{2}
$$

$$
\pi\left(\mathcal{G}_{1}(X) ; T\right)=G_{1} *_{h^{-1}} \sigma\left(G_{e}\right) h=\tau\left(G_{e}\right)\left(\bar{G}_{2}\right) .
$$

These presentations define the same group in different generators, and $\bar{x}=x^{h}$, for $x \in G_{2}$ and corresponding $\bar{x} \in \bar{G}_{2}$.

Similarly, in the case of foldings we choose $T$ so that the edge corresponding to $C$ belongs to $T$.

2.5. Freely decomposable groups. Recall that a group $G$ is freely decomposable if it is isomorphic to a non-trivial free product (in which there are at least two non-trivial factors). Otherwise, $G$ is called freely indecomposable. A free decomposition

$$
G=G_{1} * \cdots * G_{n} * F(Y)
$$

is called Grushko's decomposition if all the factors $G_{1}, \ldots, G_{n}$ are non-cyclic freely indecomposable groups, and $F(Y)$ is a free group with basis $Y$ (pehaps empty). If there is another Grushko's decomposition

$$
G=H_{1} * \cdots * H_{m} * F(Z)
$$

, then $n=m$, corresponding factors $G_{i}$ and $H_{i}$ (after reordering) are conjugated, and $|Z|=|Y|$.

Definition 2.3. Let $G$ be a group and $H$ be a subgroup of $G$. We say that $G$ has a non-trivial free decomposition modulo $H$, if

$$
G \simeq G_{1} * G_{2}
$$

$H \leq G_{1}$, and $G_{2} \neq 1$.

Now we generalize the definition above.

Definition 2.4. Let $G$ be a group and $K_{1}, \ldots, K_{n}$ be subgroups of $G$. We say that $G$ has a non-trivial free decomposition modulo $K_{1}, \ldots, K_{n}$, if

$$
G \simeq G_{1} * G_{2}
$$

$K_{1} \leq G_{1}$, and for each $i$ there exists $g_{i} \in G$ such that $K_{i}^{g_{i}} \leq G_{1}$.

LEMMA 2.5. Let $G$ be a group with a non-trivial splitting $D$ of $G$. If $D$ contains an edge with the trivial associated group then $G$ is decomposable into a non-trivial free product. 
Proof. Let $D=\mathcal{G}(X)$ be a non-trivial splitting of $G$ with an edge with the trivial associated subgroup. Denote by $X^{*}$ the subgraph of $X$ formed by all vertices in $X$ and all edges in $X$ whose associated groups are non-trivial. Assume, first, that $X *$ is not connected and $Y_{1}, \ldots, Y_{n}(n \geq 2)$ are the connected components of $X^{*}$. By collapsing in $D$ every subgraph $Y_{i}$ into a single vertex, say $v_{i}$, with the associated group $\pi_{1}\left(Y_{i}\right)$ one gets a graph of groups $Z$ in which all the edge groups are trivial. Since all the vertex groups in $D$ are nontrivial the groups $\pi_{1}\left(Y_{i}\right)$ are non-trivial. By Lemma 2.1 the group $\pi_{1}(Z)$ is the free product of its vertex groups and a free group, so it is freely decomposable. Observe, that $G \simeq \pi_{1}(Z)$ since collapses preserve the fundamental groups. Therefore $G$ is freely decomposable.

Suppose now that $X^{*}$ is connected. In this case a given maximal subtree $T$ of $X^{*}$ is also a maximal subtree of $X$. If $e$ is an edge in $X$ with the trivial associated subgroup $G_{e}$ then $e \notin T$, so $t_{e} \neq 1$ in $G=\pi_{1}(X)$. Since $G_{e}=1$ the infinite cyclic group $\left\langle t_{e}\right\rangle$ is a free factor of $G$. Hence $G$ is freely decomposable. This completes the proof of the lemma.

Definition 2.6. Let $G$ be a group and $\mathcal{K}=\left\{K_{1}, \ldots, K_{n}\right\}$ be a set of subgroups of $G$. We say that a free decomposition of $G$

$$
G \simeq G_{1} * \cdots * G_{k}
$$

is compatible with $\mathcal{K}$ if each subgroup in $\mathcal{K}$ is a conjugate of a subgroup of one of the factors $G_{j}$. Denote by $\mathcal{K}_{j}$ the set of all conjugates of subgroups in $\mathcal{K}$ which belong to $G_{j}$. This decomposition is called reduced if none of the $G_{j}$ has a non-trivial compatible free decomposition modulo $\mathcal{K}_{j}$.

Proposition 2.7. Let

$$
G=G_{1} * \cdots * G_{n} * F(Y)=G=H_{1} * \cdots * H_{m} * F(Z),
$$

be two compatible with $\mathcal{K}$ reduced free decompositions, then $n=m$, corresponding factors $G_{i}$ and $H_{i}$ (after reordering) are conjugated, and $|Z|=|Y|$.

Proof. Consider a Bass-Serre tree $T$ corresponding to the first decomposition. Subgroups from $\mathcal{K}$ fix some vertices of this tree. Each subgroup $H_{i}$ acts on $T$, and, since it does not have a free decomposition compatible with $\mathcal{K}$, fixes a vertex of this tree. Therefore it is conjugated into some factor, say $G_{i}$, of the first decomposition. Conversely, each $G_{i}$ is conjugated into some $H_{j}$. Each factor $H_{i}, G_{i}$ is malnormal, therefore $H_{i}$ and $G_{i}$ are conjugated. The normal closure generated by $H_{1} * \cdots * H_{m}$ and by $G_{1} * \cdots * G_{m}$ is the same, therefore $|Y|=|Z|$.

REMARK 1. In the notations above, if

$$
G \simeq G_{1} * \cdots * G_{k}
$$

is a compatible with $\mathcal{K}$ decomposition of $G$, then we will always assume (taking a conjugation of $G$ if necessary and renaming subgroups $G_{i}$ ) that $K_{1} \leq G_{1}$.

Definition 2.8. Let $G$ be a group and $\mathcal{K}=\left\{K_{1}, \ldots, K_{n}\right\}$ be a set of subgroups of $G$. An abelian splitting $D$ is called a splitting modulo $\mathcal{K}$ if all subgroups from $\mathcal{K}$ are conjugated into vertex groups in $D$.

2.6. Splittings of finitely generated fully residually free groups. The following result follows immediately from Theorem 1.7

THEOREM 2.9. Every freely indecomposable non-abelian group from $\mathcal{F}$ has an essential $\mathbb{Z}$-splitting. 
2.7. Elliptic and hyperbolic subgroups. If $H$ and $K$ are subgroups of a group $G$, we say that $H$ can be conjugated into $K$ if it is a conjugate of a subgroup of $K$.

An element $g \in G$ [a subgroup $H \leq G$ ] is called elliptic in a given splitting of $G$ if $g$ [correspondingly, $H$ ] can be conjugated into a vertex group, and hyperbolic otherwise.

Lemma 2.10. 67 Let $G$ be a freely indecomposable group. If $D_{i}$ is an elementary $\mathbb{Z}$-splitting of $G$ with the edge group $C_{i},(i=1,2)$, then $C_{1}$ is hyperbolic (elliptic) in $D_{2}$ if and only if $C_{2}$ is hyperbolic (elliptic) in $D_{1}$.

A pair of elementary $\mathbb{Z}$-splittings $D_{i}(i=1.2)$ is called intersecting if they form a hyperbolic-hyperbolic pair, namely $C_{1}$ is hyperbolic with respect to $D_{2}$ and $C_{2}$ is hyperbolic with respect to $D_{1}$.

Lemma 2.11. Let $G \in \mathcal{F}$ and $N$ a maximal abelian non-cyclic subgroup of $G$. Then the following holds:

(1) If $G=A *_{C} B$ is an abelian splitting of $G$ then $N$ is elliptic in this splitting;

(2) If $G=A *_{C}$ is an abelian splitting of $G$ then one of the following holds:

a) $N$ is elliptic in this splitting;

b) $C$ is the centralizer $C_{A}(v)$ of some element $v \in A, C \leq N^{g}$ for some $g \in G$, and $G=A *_{C} N^{g}$ is an extension of the centralizer $C$.

Proof. The first statement follows from the description of commuting elements in a free product with amalgamation (see, for example, [50]). The second one is a direct corollary of Lemma 2 and Theorem 5 from $\mathbf{2 4}$.

Definition 2.12. An abelian splitting $D$ of a group $G$ is called normal if all maximal abelian non-cyclic subgroups of $G$ are elliptic in $D$. By $\mathcal{D}(G)$ we denote the set of all normal splittings of $G$. Denote by $\mathcal{D}_{F}(G)$ the class of all normal splittings of $G$ such that $F$ belongs to a vertex group.

2.8. Quadratically hanging subgroups. Let $D=(G,(\mathcal{G}(X) ; T), \phi)$ be an abelian splitting of $G$. A vertex group $Q=G_{v}, v \in V$ is called quadratically hanging in $D$ (in short, QH-subgroup in $D$ ), if the following conditions hold:

1) $Q$ admits one of the following presentations

$$
\left\langle p_{1}, \ldots, p_{m}, a_{1}, \ldots, a_{g}, b_{1}, \ldots, b_{g} \mid \prod_{j=1}^{g}\left[a_{i}, b_{i}\right] \prod_{k=1}^{m} p_{k}\right\rangle, g \geq 0, m \geq 1,
$$

and if $g=0$, then $m \geq 4$,

$$
\left\langle p_{1}, \ldots, p_{m}, a_{1}, \ldots, a_{g} \mid \prod_{j=1}^{g} a_{i}^{2} \prod_{k=1}^{m} p_{k}\right\rangle, g \geq 1, m \geq 1,
$$

(in particular, $Q$ is a free group);

2) for every edge $e \in E$ outgoing from $v$, the edge group $G_{e}$ is conjugate to one of the subgroups $\left\langle p_{i}\right\rangle, i=1, \ldots, m$.

3) for each $p_{i}$ there is an edge $e_{i} \in E$ outgoing from $v$ such that $G_{e_{i}}$ is a conjugate of $\left\langle p_{i}\right\rangle$. 
Notice that for a freely indecomposable group $G$ property 3) is automatically satisfied. Moreover, using slidings one can modify the splitting $D$ in such a way that there are exactly $m$ outgoing edges $e_{1}, \ldots, e_{m}$ from the QH-vertex $Q$ and $\sigma\left(G_{e_{i}}\right)=\left\langle p_{i}\right\rangle$ for each edge $e_{i}$.

A QH-subgroup $Q$ is called a maximal $Q H$-subgroup (in short, MQH-subgroup) if for every elementary abelian splitting $D$ of $G$ ( where $G=A *_{C} B$ or $G=A *_{C}$ ) either $Q$ is elliptic in $D$, or the edge group $C$ can be conjugated into $Q$, in which case $D$ is inherited from the $\mathbb{Z}$-splitting of $Q$ along $C$.

Non-QH non-abelian vertex groups of $D$ are called rigid.

2.9. QH-subgroups and quadratic equations. Let $D$ be a splitting of $G$. One can choose the maximal subtree $T$ of the graph of groups $\Gamma$ in $D$ as follows. Let $T_{1}$ be a maximal subforest of the subgraph of $\Gamma$ spanned by non-QH non-abelian vertex groups. Then one can extend $T_{1}$ to a maximal subforest $T_{2}$ of the subgraph of $\Gamma$ spanned by all non-QH vertex groups, and then extend $T_{2}$ to a maximal tree $T$.

Orientable case. Let an MQH subgroup $Q$ in $D$ be given by a presentation

$$
\prod_{i=1}^{n}\left[x_{i}, y_{i}\right] p_{1} \ldots p_{m}=1
$$

We may assume (see Section[2.8) that there are exactly $m$ outgoing edges $e_{1}, \ldots, e_{m}$ from the QH-vertex $Q$ and $\sigma\left(G_{e_{i}}\right)=\left\langle p_{i}\right\rangle$ for each edge $e_{i}$. Denote also $\tau\left(G_{e_{i}}\right)=$ $\left\langle c_{i}\right\rangle$.

In the standard presentation of $G$, by generators and relations as the fundamental group of $D$, the relations corresponding to $\mathrm{QH}$-vertex $Q$ have the following form

$$
\prod_{i=1}^{n}\left[x_{i}, y_{i}\right] p_{1} \ldots p_{m}=1, c_{i}^{z_{i}}=p_{i}, i=1, \ldots, m,
$$

where $z_{i}=1$ if $e_{i} \in T$, and $e_{m} \in T$. Excluding $p_{i}$ from the relations above one gets the relation

$$
\prod_{i=1}^{n}\left[x_{i}, y_{i}\right] c_{1}^{z_{1}} \ldots c_{m-1}^{z_{m-1}} c_{m}=1
$$

in the presentation of $G$. We refer to this relation as to the quadratic relation in $G$ corresponding to $Q$.

Conversely, if $H \in \mathcal{F}$ and $c_{1}, \ldots, c_{m} \in H$ then the quotient group

$$
G=H\left[x_{1}, y_{1}, \ldots, x_{n}, y_{n}, z_{1}, \ldots, z_{m-1}\right] / n c l\left(\prod_{i=1}^{n}\left[x_{i}, y_{i}\right] c_{1}^{z_{1}} \ldots c_{m-1}^{z_{m-1}} c_{m}\right)
$$

can be represented as the fundamental group of a graph of groups with a $\mathrm{QH}$ vertex $Q$ given by the presentation

$$
\prod_{i=1}^{n}\left[x_{i}, y_{i}\right] p_{1} \ldots p_{m}=1
$$

with outgoing edges $e_{i}, i=1, \ldots, m$, such that $\sigma\left(G_{e_{i}}\right)=\left\langle p_{i}\right\rangle$ and $\tau\left(G_{e_{i}}\right)=\left\langle c_{i}\right\rangle$ for each edge $e_{i}$. 
Every homomorphism $\phi: G \rightarrow F$ gives rise to a solution in $F$ of the quadratic equation (corresponding to $Q$ ):

$$
\prod_{i=1}^{n}\left[x_{i}, y_{i}\right] c_{1}^{\phi z_{1}} \ldots c_{m-1}^{\phi z_{m-1}} c_{m}^{\phi}=1 .
$$

Non-orientable case. If an MQH subgroup $Q$ in $D$ is given by

$$
x_{1}^{2} \ldots x_{n}^{2} p_{1} \ldots p_{m}=1, c_{i}^{z_{i}}=p_{i}, i=1, \ldots, m,
$$

where $z_{i}=1$ if $e_{i} \in T$, and $e_{m} \in T$. Then, similarly to the orientable case, in the presentation of $G$ (relative to $D$ ) the subgroup $Q$ inputs a quadratic relation

$$
x_{1}^{2} \ldots x_{n}^{2} c_{1}^{z_{1}} \ldots c_{m-1}^{z_{m-1}} c_{m}=1 .
$$

The converse is also true, so the quotient group

$$
G=H\left[x_{1}, \ldots, x_{n}, z_{1}, \ldots, z_{m-1}\right] / n c l\left(x_{1}^{2} \ldots x_{n}^{2} c_{1}^{z_{1}} \ldots c_{m-1}^{z_{m-1}} c_{m}\right)
$$

can be represented as the fundamental group of a graph of groups with a $\mathrm{QH}$ vertex $Q$ corresponding to the quadratic relation above. Again, homomorphisms $\phi: G \rightarrow F$ give rise to solutions of the equation

$$
x_{1}^{2} \ldots x_{n}^{2} c_{1}^{\phi z_{1}} \ldots c_{m-1}^{\phi z_{m-1}} c_{m}=1 .
$$

\subsection{Induced $Q H$-subgroups.}

Lemma 2.13. Let $G \in \mathcal{F}, \Gamma=\Gamma(X)$ a cyclic [abelian] splitting of $G$, and $Q$ a $Q H$-subgroup in $\Gamma$ associated with a vertex $v \in X$ with outgoing edges $e_{1}, \ldots, e_{m}$. Denote by $Y_{1}, \ldots, Y_{k}$ the connected components of the graph $X \backslash\left\{e_{1}, \ldots, e_{m}\right\}$ and by $P_{1}, \ldots, P_{k}$ - the fundamental groups of the graphs of groups induced from $\Gamma$ on $Y_{1}, \ldots, Y_{k}$. If $H$ is a finitely generated non-cyclic subgroup of $G$ then one of the following conditions holds:

(1) $H$ is a nontrivial free product;

(2) $H \leq P_{i}^{g}$ for some $g \in G$ and $1 \leq i \leq k$;

(3) $H$ is freely indecomposable, and for some $g \in G$ the subgroup $H \cap Q^{g}$ has finite index in $Q^{g}$. In this event $H \cap Q^{g}$ is a $Q H$-vertex group in $H$.

If $H_{Q}=H \cap Q$ is non-trivial and has infinite index in $Q$, then $H_{Q}$ is a free product of some conjugates of $p_{1}^{\alpha_{1}}, \ldots, p_{m}^{\alpha_{m}}, p^{\alpha}$ and a free group $F_{1}$ (maybe trivial) which does not intersect any conjugate of $\left\langle p_{i}\right\rangle$ for $i=1, \ldots, m$.

Proof. We first prove the lemma for a freely indecomposable group $G$. Denote by $D$ the splitting of $G$ obtained from the splitting $\Gamma$ by collapsing all the edges in $X$ not adjacent to the vertex $v$. Let $D_{H}$ be the splitting of $H$ induced by $D$. Suppose some conjugate of $Q$ has a non-trivial intersection with $H$. Without loss of generality we can assume it is $Q$ itself. Put $Q_{H}=Q \cap H$. The group $Q_{H}$ is finitely generated as the intersection of two finitely generated subgroups of a fully residually free group (see Lemma 3.13 in Section 31) and, therefore, finitely presented.

Suppose that $Q$ has a presentation

$$
Q=\left\langle x_{1}, y_{1}, \ldots, x_{n}, y_{n}, p_{1}, \ldots, p_{m}, p \mid \prod_{i=1}^{n}\left[x_{i}, y_{i}\right] \prod_{i=1}^{m} p_{i} p=1\right\rangle .
$$

Let

$$
\hat{Q}=\left\langle\hat{x}_{1}, \hat{y}_{1}, \ldots, \hat{x}_{n}, \hat{y}_{n}, \hat{p}_{1}, \ldots, \hat{p}_{m}, \hat{p} \mid \prod_{i=1}^{n}\left[\hat{x}_{i}, \hat{y}_{i}\right] \prod_{i=1}^{m} \hat{p}_{i} \hat{p}=1\right\rangle
$$


Denote by $\bar{Q}$ a group generated by $x_{i}, y_{i}, \hat{x}_{i}, \hat{y}_{i}, p_{i}, \hat{p}_{i}, p, \hat{p}, t_{i}$ and given by defining relations

$$
\begin{gathered}
\prod_{i=1}^{n}\left[x_{i}, y_{i}\right] \prod_{i=1}^{m} p_{i} p=1, \\
\prod_{i=1}^{n}\left[\hat{x}_{i}, \hat{y}_{i}\right] \prod_{i=1}^{m} \hat{p}_{i} \hat{p}=1, \\
p_{i}^{t_{i}}=\hat{p}_{i}, \quad p=\hat{p} .
\end{gathered}
$$

The group $\bar{Q}$ is a closed surface group. It is also a fundamental group of a graph of groups with two vertices $w$ and $\hat{w}$ and $m+1$ edges $e_{0}, \ldots, e_{m}$ joining these vertices. The vertex groups are $G_{w}=Q, G_{\hat{w}}=\hat{Q}$ and the edge groups are $G_{e_{0}}=\langle p\rangle, G_{e_{i}}=\left\langle p_{i}\right\rangle$.

There are two possibilities.

1. $Q_{H}$ does not intersect non trivially a conjugate of any subgroup $\left\langle p_{i}\right\rangle, i=$ $1, \ldots m$ and $\langle p\rangle$. In this case $H$ is a nontrivial free product, because $Q_{H}$ is a nontrivial vertex group in $D_{H}$ with all the adjacent edge groups trivial.

2. $Q_{H}$ intersects non trivially $\left\langle p_{i}\right\rangle^{g}$. Without loss of generality we can suppose that $Q_{H} \cap\langle p\rangle^{g}$ is non trivial. Taking $Q_{H}^{g^{-1}}$ instead of $Q_{H}$ we can suppose that $Q_{H}$ intersects non-trivially the subgroup $\langle p\rangle$. Consider a graph of groups $\Gamma$ with two vertices $v$ and $\hat{v}$, and vertex groups $Q_{H}$, and the double of this group $\hat{Q}_{H} \leq \hat{Q}$. The outgoing edges from $v$ correspond to all possible intersections of the conjugates of $\left\langle p_{1}\right\rangle, \ldots,\left\langle p_{m}\right\rangle,\langle p\rangle$ in $Q$ with $Q_{H}$ which are not conjugated in $Q_{H}$. The outgoing edges from $\hat{v}$ are their doubles. To obtain $\Gamma$, we identify each edge with its double. Let $\bar{Q}_{H}$ be the fundamental group of $\Gamma$ with stable letters $s_{1}, \ldots, s_{n}$. Suppose the edge groups of $\bar{Q}_{H}$ conjugated into $\left\langle p_{i}\right\rangle$ are $\left\langle p_{i}^{\alpha_{j} g_{j}}\right\rangle, j=1, \ldots, k_{i}$, where $g_{j}$ are representatives of $\left(\left\langle p_{i}\right\rangle, Q_{H}\right)$ double cosets in $Q$, and the edge group conjugated into $\langle p\rangle$ are $\left\langle p^{\alpha_{l} g_{l}}\right\rangle, l=1, \ldots, k_{0}$ ( $\alpha$ 's are natural numbers). Relations of $\bar{Q}_{H}$ consist of relations of $Q_{H}$ and $\hat{Q}_{H}$ and relations

$$
p^{\alpha_{l} g_{l} s_{0 l}}=\hat{p}^{\alpha_{l} \hat{g}_{l}}
$$

where $g_{1}=s_{01}=1$, and

$$
p_{i}^{\alpha_{j} g_{j} s_{i j}}=\hat{p}_{i}^{\alpha_{j} \hat{g}_{j}} .
$$

We will show now that $\bar{Q}_{H}<\bar{Q}$. Notice first, that $p_{i}^{g_{j}}$ is conjugated to $\hat{p}_{i}^{\hat{g}_{j}}$ by $g_{j}^{-1} t_{i} \hat{g}_{j}$. Consider a homomorphism $\phi: \bar{Q}_{H} \rightarrow \bar{Q}$ that extends the natural embedding of $Q_{H}$ into $Q$ and $\hat{Q}_{H}$ into $\hat{Q}$ and such that $s_{0 l}^{\phi}=g_{l}^{-1} \hat{g}_{l}, s_{i j}^{\phi}=g_{j}^{-1} t_{i} \hat{g}_{j}$. It is a homomorphism, because it preserves the relations of $\bar{Q}_{H}$.

We have to show that it is a monomorphism. Take some element $r \in \bar{Q}_{H}$ in the HNN reduced form $r=h_{0} s_{i_{1}}^{\epsilon_{1}} h_{1} \ldots s_{i_{n}}^{\epsilon_{n}} h_{n},(n \geq 0)$, where $h_{i} \in Q_{H} *\left\langle p^{\left.\alpha_{0}=\hat{p}^{\alpha} 0\right\rangle}\right.$ $\hat{Q}_{H}, \epsilon_{i} \in\{1,-1\}$. Since $r$ is in the reduced form, there is no consecutive subsequence $s_{i j}^{-1}, h_{k}, s_{i j}$ with $h_{k} \in\left\langle p_{i}^{\alpha_{i j} g_{i j}}\right\rangle$ or $s_{i j}, h_{k}, s_{i j}^{-1}$ with $h_{k} \in\left\langle\hat{p}_{i}^{\alpha_{i j} \hat{g}_{i j}}\right\rangle$. Suppose, first, that $n=0, r=h_{0}$. Then $h_{0}=q_{1} \hat{q}_{2} \ldots q_{n-1} \hat{q}_{n}$, where $q_{i} \notin\left\langle p^{\alpha_{01}}\right\rangle$ and $\hat{q}_{i} \notin\left\langle\hat{p}^{\alpha_{01}}\right\rangle$. This is a reduced form in the amalgamated product $Q_{H} *\left\langle p^{\alpha_{0}}=\hat{p}^{\alpha_{0}}\right\rangle \hat{Q}_{H}$. This element considered as an element of $Q *\langle p=\hat{p}\rangle \hat{Q}$ is also in the reduced form, therefore is nontrivial. Since $Q *{ }_{\langle p=\hat{p}\rangle} \hat{Q} \leq \bar{Q}, r^{\phi} \neq 1$. Suppose now that $n>0$. To obtain $r^{\phi}$ we substitute in $r$ elements $s_{0 l}^{\phi}=g_{l}^{-1} \hat{g}_{l}, s_{i j}^{\phi}=g_{j}^{-1} t_{i} \hat{g}_{j}$ instead of $s_{0 l}, s_{i j}$. We have 
to show that $r^{\phi}$ is in the reduced form in $\bar{Q}$. Consider first the case, when $r$ does not contain any $s_{i j}, i \neq 0$, therefore, $r^{\phi}$ does not contain any $t_{i}$. In this case

$$
r^{\phi}=h_{0}\left(g_{i_{1}}^{-1} \hat{g}_{i_{1}}\right)^{\epsilon_{1}} h_{1} \ldots\left(g_{i_{n}}^{-1} \hat{g}_{i_{n}}\right)^{\epsilon_{n}} h_{n},
$$

$(n \geq 0)$, where $h_{i} \in Q_{H} *\left\langle p^{\alpha_{0}}=\hat{p}^{\left.\alpha_{0}\right\rangle} \hat{Q}_{H}, \epsilon_{i} \in\{1,-1\}\right.$. This is an element from $Q *\langle p=\hat{p}\rangle \hat{Q}$. If we write $h_{1}, \ldots, h_{n}$ in reduced form, we can see how to write $r^{\phi}$ in reduced form. Without loss of generality we can suppose that $\epsilon_{1}=-1$. Since $g_{i j} \in Q \backslash Q_{H}$, the syllable length of $\left(g_{i_{1}}^{-1} \hat{g}_{i_{1}}\right)^{\epsilon_{1}} h_{1}\left(g_{i_{2}}^{-1} \hat{g}_{i_{2}}\right)^{\epsilon_{2}}$ can become less than three only in the case $\epsilon_{2}=1, h_{1} \in Q_{H}$ and $g_{i_{1}} h_{1} g_{i_{2}}^{-1} \in\langle p\rangle$. Suppose this is the case. Then $Q_{H}$ contains $h_{1}=g_{i_{1}}^{-1} p^{k} g_{i_{2}}, p^{\alpha}, g_{i_{1}}^{-1} p^{\alpha_{i_{1}}} g_{i_{1}}, g_{i_{2}}^{-1} p^{\alpha_{i_{2}}} g_{i_{2}}$. Therefore it contains $h_{1} g_{i_{2}}^{-1} p^{\alpha_{i_{1}}-k} g_{i_{1}}$. Then $g_{i_{1}}$ and $g_{i_{2}}$ represent the same double coset and thus equal, which implies that corresponding stable letters $s_{0 l}$ are the same and $r=h_{0} s_{0 l}^{-1} h_{1} s_{0 l} \cdots h_{n}$ is not in reduced form. This contradicts to the assumption. Suppose now $r$ does contain $s_{i j}$ for some $i>1$. Suppose that we have a reducible sequence $t_{i}^{-1} p_{i}^{k} t_{i}$ in $r^{\phi}$. This can only appear from the sequence $s_{i j_{1}}^{-1} h s_{i j_{2}}$ in reduced form of $r$. We can take for definiteness $j_{1}=1, j_{2}=2$. Then $\left(s_{i 1}^{-1} h s_{i 2}\right)^{\phi}=\hat{g}_{i_{1}}^{-1} t_{i}^{-1} g_{i_{1}} h g_{i_{2}}^{-1} t_{i} \hat{g}_{i_{2}}$. As in the previous case we have $g_{i_{1}} h g_{i_{2}}^{-1}=p_{i}^{k}$, and $Q_{H}$ contains $h=g_{i_{1}}^{-1} p_{i}^{k} g_{i_{2}}$. This implies $s_{1}=s_{2}$ and $r$ is not in reduced form, that contradicts the assumption.

Either $\bar{Q}_{H}$ is a closed surface group (in this case $\bar{Q}_{H}$ has finite index in $\bar{Q}$ and $Q_{H}$ is of finite index in $Q$ ), or $\bar{Q}_{H}$ is free.

Suppose $\bar{Q}_{H}$ is free. Denote the elements $p_{i}^{\alpha_{i j} g_{i j}}$ by $q_{0}, \ldots, q_{n}$, where $q_{0}$ is a power of $p$, and rewrite the corresponding relations in the form $q_{0}=\hat{q}_{0}, q_{i}^{s_{i}}=\hat{q}_{i}$. We will prove that for some $h_{1}, \ldots, h_{n} \in Q_{H}$ the product $\left\langle q_{0}\right\rangle *\left\langle q_{1}\right\rangle^{h_{1}} * \cdots *\left\langle q_{n}\right\rangle^{h_{n}}$ is a free factor of $Q_{H}$. Consider the following free groups.

$$
R_{n}=Q_{H} *\left\langle r_{1}\right\rangle * \cdots\left\langle r_{n}\right\rangle, \hat{R}_{n}=\hat{Q}_{H} *\left\langle\hat{r}_{1}\right\rangle * \cdots\left\langle\hat{r}_{n}\right\rangle .
$$

Let $\bar{R}_{n}$ be the amalgamated product of $R_{n}$ and $\hat{R}_{n}$ amalgamated along subgroups $\left\langle q_{0}, q_{1}^{r_{1}}, \ldots, q_{n}^{r_{n}}\right\rangle$ and $\left\langle\hat{q}_{0}, \hat{q}_{1}^{\hat{r}_{1}}, \ldots, \hat{q}_{n}^{\hat{r}_{n}}\right\rangle$ such that $q_{0}=\hat{q}_{0}, q_{i}^{r_{i}}=\hat{q}_{i}^{\hat{r}_{i}}$. The group $\bar{Q}_{H}$ is embedded into $\bar{R}_{n}$. More precise, $\bar{Q}_{H} *\left\langle r_{1}\right\rangle * \cdots\left\langle r_{n}\right\rangle$ is isomorphic to $\bar{R}_{n}$. Indeed, a mapping $\theta$ that maps $Q_{H}$ and $\hat{Q}_{H}$ identically onto corresponding subgroups of $\bar{R}_{n}$, maps $s_{i}$ to $r_{i}^{-1} \hat{r}_{i}$ and $r_{i}$ to $r_{i}$ can be extended to an isomorphism.

The group $\bar{R}_{n}$ is an amalgamated product of two copies of the free group $Q_{H} *\left\langle r_{1}\right\rangle * \cdots *\left\langle r_{n}\right\rangle$ along the same subgroup. By a result of Swan (see [64, Lemma 7.1) such a group is free if and only if the amalgamated subgroup is a free factor.

Therefore $Q_{H} *\left\langle r_{1}\right\rangle * \cdots *\left\langle r_{n}\right\rangle=\left\langle q_{0}\right\rangle *\left\langle q_{1}^{r_{1}}\right\rangle * \cdots *\left\langle q_{n}^{r_{n}}\right\rangle * F_{0}$ for some free group $F_{0}$. Each subgroup $\left\langle q_{i}\right\rangle$, in particular $\left\langle q_{0}\right\rangle$, is a free factor in $Q_{H}$ by the same result of Swan. Let $Q_{H}=Q_{H}^{\prime} *\left\langle q_{0}\right\rangle$. Considering $\bar{R}_{n}$ for $n=1$ we obtain that $\left\langle q_{0}\right\rangle * Q_{H}^{\prime} *\left\langle r_{1}\right\rangle=\left\langle q_{0}\right\rangle *\left\langle q_{1}^{r_{1}}\right\rangle * F_{0}$. Denote this group by $T$. We want to show that $\left\langle q_{0}\right\rangle *\left\langle q_{1}\right\rangle^{h}$ is a free factor of $Q_{H}$ for some $h \in Q_{H}$. Fix a basis of $T$ that includes $q_{0}$, some basis of $Q_{H}^{\prime}$ and $r_{1}=r$, and represent elements of $\left\langle q_{1}^{r}\right\rangle$ and $F_{0}$ in this basis. We will now be considering bases of $T$ containing $q_{0}, q_{1}^{r}$. We order bases of $T$ containing $q_{0}, q_{1}^{r}$ and some elements $\bar{z}=\left\{z_{1}, \ldots, z_{k}\right\}$ the following way: let $n_{\bar{z}}=\left(n_{0}, \ldots, n_{i}, \ldots\right)$, where $n_{i}$ is the number of elements in $\bar{z}$ containing $i$ occurrences of $r^{ \pm 1}$. One basis is smaller than the other if the tuple $n_{\bar{z}}$ for it is larger in the left lexicographic order. Let $\ell\left(z_{i}\right)$ be the number of occurrences of $r^{ \pm 1}$ 
in $z_{i}$. Applying Nielsen transformations that do not change $q_{0}$ and $q_{1}^{r}$ to a minimal basis of $T$ we turn the set of basis elements that do not contain $r^{ \pm 1}$ into a Nielsen set. We call such a basis a strongly minimal. If, for some $z_{i}, \ell\left(q_{1}^{r} z_{i}^{ \pm 1}\right)<2$, then either $q_{1}^{r}$ is completely cancelled, and this contradicts the minimality, or $\ell\left(z_{i}\right)=1$ and $q_{1}^{r z_{i}^{ \pm 1}}$ is a free factor in $Q_{H}^{\prime}$, therefore $\left\langle q_{0}\right\rangle *\left\langle q_{1}\right\rangle^{h}$ is a free factor in $Q_{H}$ and we do not have to prove anything.

We claim now that by applying Nielsen transformations that do not change $q_{0}$ and $q_{1}^{r}$ to a strongly minimal basis of $T$ we can turn this basis into a basis with the following properties: (1) for any two basis elements $y_{1}, y_{2}, \ell\left(y_{1} y_{2}^{ \pm 1}\right) \geq \ell\left(y_{1}\right)$; (2) if for a basis element $z$ the number $\ell(z)$ is odd, then the middle occurrence of $r^{ \pm 1}$ in $z$ is not cancelled in any product of basis elements; (3) in any product of basis elements, elements with an even number of occurrences of $r^{ \pm 1}$ greater than two have either one of the middle occurrences of $r^{ \pm 1}$ uncancelled or, if all occurrences of $r^{ \pm 1}$ are cancelled, one of the middle letters not cancelled. (4) in any product of $q_{1}^{r}$ and elements $z_{i}$, such that each $z_{i}$ either doesn't contain $r^{ \pm 1}$ or contains two occurrences of $r^{ \pm 1}$, the first and last letter $r^{ \pm 1}$ cannot cancel. The first two properties we already have for a strongly minimal basis. The proof of (3) is just a repetition of the proof of the same property in Nielsen's theorem. We will now prove (4). Suppose that the first and last letters $r^{ \pm 1}$ are cancelled in the product of the form $q_{1}^{r} z_{i_{1}}^{\epsilon_{1}} \ldots z_{i_{j}}^{\epsilon_{j}}$. If $z_{i_{j}} \neq z_{i}, i=i_{1}, \ldots, i_{j-1}$ we can replace $z_{i_{j}}$ by $q_{1}^{r} z_{i_{1}}^{\epsilon_{1}} \ldots z_{i_{j}}^{\epsilon_{j}}$ and obtain a smaller basis which contradicts the minimality of the basis $q_{0}, q_{1}^{r}, z_{1}, \ldots z_{k}$. Suppose $z_{i_{j}}$ appears more than once in the product $q_{1}^{r} z_{i_{1}}^{\epsilon_{1}} \ldots z_{i_{j}}^{\epsilon_{j}}$. Suppose $z_{i_{j}}^{\epsilon_{j}}$ ends with $r w$, where $w \in Q_{H}$. Then $w$ cannot be expressed in terms of basis elements of length zero and $w \neq 1$ because the expression is nontrivial. Let $z_{i_{t}}^{\epsilon_{t}}$ be the first element from the right in the expression $q_{1}^{r} z_{i_{1}}^{\epsilon_{1}} \ldots z_{i_{j}}^{\epsilon_{j}}$ that ends with $r w$, where $w \in Q_{H}$ and does not begin with $w^{-1} r^{-1}$ (if $z_{i_{j}}^{\epsilon_{j}}$ does not begin with $w^{-1} r^{-1}$, then $t=j$ ). Multiplying all the other basis elements of length 2 that end with $r w$ by $z_{i_{t}}^{ \pm 1}$ we will obtain a new basis such that the unique element of length two that ends with $r w$ or begins with $w^{-1} r^{-1}$ (but not both)is $z_{i_{t}}$. Since the expression $q_{1}^{r} z_{i_{1}}^{\epsilon_{1}} \ldots z_{i_{j}}^{\epsilon_{j}}$ equals $w$, in the new basis it ends with $z_{i_{t}}$, and $z_{i_{t}}$ appears only once. Replacing $z_{i_{t}}$ by this expression, we obtain a basis smaller than the one we began with, contradicting minimality. Let $q_{0}, q_{1}^{r}, z_{1}, \ldots, z_{k}$ be a basis with properties (1)(4). Denote by $F_{0}^{\prime}$ the free group with basis $\left\{z_{1}, \ldots, z_{k}\right\}$. Then $r \in\left\langle q_{1}^{r}, z_{1}, \ldots, z_{k}\right\rangle$ and $T=\left\langle q_{0}\right\rangle *\left\langle q_{1}\right\rangle *\left(F_{0}^{\prime}\right)^{r^{-1}}$. Therefore $Q_{H}=\left\langle q_{0}\right\rangle *\left\langle q_{1}\right\rangle * F_{0}^{\prime \prime}$.

We can now apply induction on $n$ and prove that $Q_{H}$ is a free product of conjugates of $p_{1}^{\alpha_{1}}, \ldots, p_{m}^{\alpha_{m}}, p^{\alpha}$ belonging to $Q_{H}$ and a group $F_{1}$ which does not intersect any conjugate of $\left\langle p_{i}\right\rangle$ for $i=1, \ldots, m$. Each conjugate of $p_{j}^{\alpha}$ in $H$ can be conjugated into one of the groups $\left\langle p_{i}^{\alpha_{i}}\right\rangle$ in $Q_{H}$. This proves the second statement of the lemma.

Suppose that for any $g$ the subgroup $Q^{g} \cap H$ is either trivial or has the structure described above. Consider now the decomposition $D_{H}$. If the group $F_{1}$ is nontrivial, then $H$ is freely decomposable, because the vertex group $Q_{H}$ in $D_{H}$ is a free product, and all the edge groups belong to the other factor. If at least for one subgroup $Q^{g}$, such a group $F_{1}$ is non-trivial, then $H$ is a non-trivial free product. Suppose each non-trivial subgroup $H \cap Q^{g}$ is a free product of conjugates of some elements $p_{i}^{\alpha_{i}}, \alpha_{i} \in Z$, in $Q^{g}$. According to the Bass-Serre theory, for the group $G$ and its decomposition $D$ one can construct a tree such that $G$ acts on this tree, and 
stabilizers correspond to vertex and edge groups of $D$. Denote this Bass-Serre tree by $T_{D}$. The subgroup $H$ also acts on $T_{D}$. Let $T_{1}$ be a fundamental transversal for this action. Since $H$ is not conjugated into any of the subgroups $P_{i}$, the amalgamated product of the stabilizers of the vertices of $T_{1}$ is a free product of subgroups $H \cap P_{i}^{g}$. Therefore $H$ is either such a free product or is obtained from such a free product by a sequence of HNN extensions with associated subgroups belonging to distinct factors of the free product. In both cases $H$ is freely decomposable.

In the case when $Q_{H}$ has finite index in $Q, \bar{Q}_{H}$ has finite index in $\bar{Q}$, therefore the closed surface with the fundamental group $\bar{Q}_{H}$ is a finite cover of the closed surface with the fundamental group $\bar{Q}$. Therefore $Q_{H}$ is a QH subgroup for $H$.

Suppose now that $G=G_{1} * \cdots * G_{k} * F_{r}$ is a Grushko's decomposition of $G$, where $G_{1}, \ldots, G_{k}$ are freely indecomposable non-cyclic groups. Then any other such decomposition has the same numbers $k$ and $r$ and indecomposable factors conjugated to $G_{1}, \ldots, G_{k}$. Since $H$ is freely indecomposable, we can suppose that $H$ is contained in $G_{1}$. Let $D$ be a decomposition of $G$ as in the lemma, and $H$ does not belong to a conjugate of $P_{i}$. If the intersection $Q^{g} \cap H$ has infinite index in $Q^{g}$ for any $g \in G$, then the proof that $H$ should be freely decomposable does not require the property of $G$ being freely indecomposable. Suppose there exists $g \in G$ such that this intersection has finite index. We can suppose $g=1$, then $Q \leq G_{1}$. Then $Q$ is a vertex group in the induced decomposition of $G_{1}$, therefore it is a $\mathrm{QH}$ subgroup of $G_{1}$. Now we can apply Lemma 2.13 to $H$ and $G_{1}$ and conclude that $Q \cap H$ is a $Q H$ subgroup of $H$.

2.11. Quadratic decomposition. By Theorem 5.6 from 67 for every f. g. freely indecomposable torsion free non-surface group $H$ there exists a reduced (may be trivial) $\mathbb{Z}$-splitting $D_{\text {quadr }}$ (a quadratic decomposition of $H$ ) with the following properties:

(1) every $M Q H$ subgroup of $H$ can be conjugated to a vertex group in $D_{\text {quadr }}$; every $Q H$ subgroup of $H$ can be conjugated into one of the $M Q H$ subgroups of $H$; every vertex with a non-MQH vertex group is adjacent only to vertices with $\mathrm{MQH}$ vertex groups;

(2) if an elementary $\mathbb{Z}$-splitting $H=A *_{C} B$ or $H=A *_{C}$ is hyperbolic in another elementary $\mathbb{Z}$-splitting of $H$, then $C$ can be conjugated into some MQH subgroup;

(3) for every elementary $\mathbb{Z}$ - [abelian] splitting $H=A *_{C} B$ or $H=A *_{C}$ from $\mathcal{D}(H)$ which is elliptic in each elementary $\mathbb{Z}$ - [abelian] splitting from $\mathcal{D}(H)$, the edge group $C$ can be conjugated into a non-MQH subgroup of $D_{\text {quadr }}$

(4) if $D_{\text {quadr }}^{\prime}$ is another splitting that has properties (1)-(4), then it can be obtained from $D_{\text {quadr }}$ by slidings, conjugations, and modifying boundary monomorphisms by conjugation.

2.12. JSJ-decompositions. All elementary cyclic [abelian] splittings of a finitely presented (f.p.) torsion free freely indecomposable group are encoded in a splitting called a JSJ decomposition.

Proposition 2.14. ( 67, part of Theorem 7.1) Let $H$ be a f.p. torsion-free freely indecomposable group. There exists a reduced, unfolded $\mathbb{Z}$-splitting of $H$, called a JSJ decomposition of $H$, with the following properties. 
(1) Every $M Q H$ subgroup of $H$ can be conjugated to a vertex group in the JSJ decomposition. Every $Q H$ subgroup of $H$ can be conjugated into one of the $M Q H$ subgroups of $H$. Every non-MQH vertex group in the JSJ decomposition is elliptic in every $\mathbb{Z}$-splitting of $H$.

(2) If an elementary $\mathbb{Z}$-splitting $H=A *_{C} B$ or $H=A *_{C}$ is hyperbolic in another elementary $\mathbb{Z}$ splitting, then $C$ can be conjugated into some $M Q H$ subgroup.

We call a splitting of a finitely presented group almost reduced if vertices of valency one and two properly contain the images of the edge groups except vertices between two MQH subgroups that may coincide with one of the edge groups.

Proposition 2.15. Let $H$ be a freely indecomposable f.g. fully residually free group. There exists an almost reduced unfolded cyclic [abelian] splitting $D \in \mathcal{D}(H)$ of $H$, where the class $\mathcal{D}(H)$ was defined in Section 2.7 with the following properties:

(1) Every $M Q H$ subgroup of $H$ can be conjugated to a vertex group in D; every $Q H$ subgroup of $H$ can be conjugated into one of the $M Q H$ subgroups of $H$; non- $M Q H$ subgroups in $D$ are of two types: maximal abelian and non-abelian, every non-MQH vertex group in $D$ is elliptic in every cyclic [abelian] splitting in $\mathcal{D}(H)$.

(2) If an elementary cyclic [abelian] splitting $H=A *_{C} B$ or $H=A *_{C}$ is hyperbolic in another elementary cyclic [abelian] splitting, then $C$ can be conjugated into some $M Q H$ subgroup.

(3) Every elementary cyclic [abelian] splitting $H=A *_{C} B$ or $H=A *_{C}$ from $\mathcal{D}(H)$ which is elliptic with respect to any other elementary cyclic [abelian] splitting from $\mathcal{D}(H)$ can be obtained from $D$ by a sequence of collapsings, foldings, conjugations and modifying boundary monomorphisms by conjugation.

(4) If $D_{1} \in \mathcal{D}(H)$ is another splitting that has properties 1-2, then it can be obtained from $D$ by slidings, conjugations, and modifying boundary monomorphisms by conjugation.

We will call such a splitting a cyclic [abelian] JSJ decomposition of $H$. Similar result holds for the class of splittings $\mathcal{D}_{F}(H)$. Such a splitting will be called an cyclic (or abelian) JSJ decomposition of $H$ modulo $F$.

Proof. By Lemma 2.11 an elementary abelian non-cyclic splitting from $\mathcal{D}(H)$ is elliptic in all splittings from $\mathcal{D}(H)$. The proof of Lemma 2.1 from 67 can be repeated to show that the case when a cyclic splitting $H=A_{1} *_{C_{1}} B_{1}\left(H=A_{1} *_{C_{1}}\right)$ is hyperbolic in a non-cyclic abelian splitting $H=A_{2} *_{C_{2}} B_{2}\left(H=A_{2} *_{C_{2}}\right)$ is impossible.

To construct a decomposition $D$ with properties 1-4, we first construct a cyclic decomposition $D_{\text {cyclic }}$. We begin with $D_{\text {quadr }}$ and refine it using property (c). We take an elementary cyclic splitting from $\mathcal{D}(H)$, and if there is a non-MQH vertex group that is not elliptic in this splitting, we split this vertex group. This process stops, because $H$ is finitely presented $\mathbf{9}$. As a result we obtain a cyclic splitting of $H$. All non-MQH vertex groups of this splitting are elliptic in all cyclic splittings from $\mathcal{D}(H)$.

Every subgraph corresponding to a decomposition $H_{1} *_{C_{1}} H_{2} *_{C_{2}} \ldots H_{n}$, such that $C_{1}, \ldots, C_{n-1}$ are subgroups of a maximal abelian subgroup $M$ which is a subgroup of $H_{1}$ can be modified (using slidings) into a star of groups with $H_{1}$ 
at the center. Since all maximal abelian subgroups of $G$ are elliptic in $D$ and they are malnormal, using slidings we can transform an abelian decomposition into 2-acylindrical decomposition. Now we can refine $D_{\text {cyclic }}$ by splitting nonMQH non-abelian subgroups using non-cyclic abelian splittings from $\mathcal{D}(H)$. This procedure stops by [68, and we obtain a decomposition $D$ with properties 1-2. These properties and 2-acylindricity imply properties (3) and (4).

COROLlary 2.16. (from Theorem 1.7) Every freely indecomposable non-abelian non-surface group from $\mathcal{F}$ admits a non-degenerate cyclic [abelian] JSJ decomposition.

Let $G$ be a group and $\mathcal{K}=\left\{K_{1}, \ldots, K_{n}\right\}$ be a set of subgroups of $G$. Consider a free decomposition $G=G_{1} * \cdots * G_{\ell}$ compatible with $\mathcal{K}$ and such that each factor $G_{i}$ that contains conjugates of some $K_{j}, j=1, \ldots, n$, is freely indecomposable modulo these subgroups and does not have a non-trivial compatible free decomposition modulo them, and each factor $G_{i}$ that does not contain any conjugate of $K_{j}, j=1, \ldots, n$, is freely indecomposable. For each $G_{i}$ that does not contain conjugates of any $K_{j}$ we can consider an abelian JSJ decomposition. For each $G_{i}$ containing conjugates of some $K_{j_{1}}, \ldots, K_{j_{s}}$ one can consider splittings modulo $K_{j_{1}}, \ldots, K_{j_{s}}$ and introduce the notion of a JSJ decomposition modulo $K_{j_{1}}, \ldots, K_{j_{s}}$. This decomposition may be degenerate. Similarly to Proposition 2.15 one can prove.

Proposition 2.17. Let $H$ be a freely indecomposable f.g. fully residually free group modulo $\mathcal{K}=\left\{K_{j_{1}}, \ldots, K_{j_{s}}\right\}$ such that there is no non-trivial compatible free decomposition of $H$ modulo $\mathcal{K}$. There exists an abelian splitting $D \in \mathcal{D}(H)$ of $H$ modulo $\mathcal{K}$ (possibly degenerate), where the class $\mathcal{D}(H)$ was defined in Section 2.7 . with the following properties:

(1) Every $M Q H$ subgroup of $H$ modulo $\mathcal{K}$ can be conjugated to a vertex group in $D$; every $Q H$ subgroup of $H$ modulo $\mathcal{K}$ can be conjugated into one of the $M Q H$ subgroups of $H$ modulo $\mathcal{K}$; non- $M Q H$ subgroups in $D$ are of two types: maximal abelian and non-abelian, every non-MQH vertex group in $D$ is elliptic in every abelian splitting in $\mathcal{D}(H)$ modulo $\mathcal{K}$.

(2) If an elementary abelian splitting $H=A *_{C} B$ or $H=A *_{C}$ modulo $\mathcal{K}$ is hyperbolic in another elementary abelian splitting modulo $\mathcal{K}$, then $C$ can be conjugated into some MQH subgroup.

(3) Every elementary abelian splitting modulo $\mathcal{K}, H=A *_{C} B$ or $H=A *_{C}$ from $\mathcal{D}(H)$ which is elliptic with respect to any other elementary abelian splitting modulo $\mathcal{K}$ from $\mathcal{D}(H)$ can be obtained from $D$ by a sequence of collapsings, foldings, conjugations and modifying boundary monomorphisms by conjugation.

(4) If $D_{1} \in \mathcal{D}(H)$ is another splitting modulo $\mathcal{K}$ that has properties 1 -2, then it can be obtained from $D$ by slidings, conjugations, and modifying boundary monomorphisms by conjugation.

To obtain an abelian JSJ decomposition of $G$ modulo $\mathcal{K}$ we first take a free decomposition as described above $G=G_{1} * \cdots * G_{\ell}$ and then an abelian JSJ decomposition of each factor $G_{i}$ modulo $\left\{K_{j_{1}}, \ldots, K_{j_{s}}\right\}$.

If $G$ is an $F$-group, we always suppose that $F \leq K_{1}$.

2.13. NTQ systems and NTQ groups. We recall now the definition of a NTQ group from 40 and 36 . 
Let $G$ be a group with a generating set $A$. A system of equations $S=1$ is called triangular quasiquadratic (shortly, TQ) if it can be partitioned into the following subsystems

$$
\begin{aligned}
S_{1}\left(X_{1}, X_{2}, \ldots, X_{n}, A\right) & =1, \\
S_{2}\left(X_{2}, \ldots, X_{n}, A\right) & =1, \\
\ldots \quad S_{n}\left(X_{n}, A\right) & =1
\end{aligned}
$$

where for each $i$ one of the following holds:

1) $S_{i}$ is quadratic in variables $X_{i}$;

2) $S_{i}=\left\{[y, z]=1,[y, u]=1 \mid y, z \in X_{i}\right\}$ where $u$ is a group word in $X_{i+1} \cup \ldots \cup X_{n} \cup A$ such that its canonical image in $G_{i+1}$ is not a proper power. In this case we say that $S_{i}=1$ corresponds to an extension of a centralizer;

3) $S_{i}=\left\{[y, z]=1 \mid y, z \in X_{i}\right\}$;

4) $S_{i}$ is the empty equation.

Define $G_{i}=G_{R\left(S_{i}, \ldots, S_{n}\right)}$ for $i=1, \ldots, n$ and put $G_{n+1}=G$. The TQ system $S=1$ is called non-degenerate (shortly, NTQ) if each system $S_{i}=1$, where $X_{i+1}, \ldots, X_{n}$ are viewed as the corresponding constants from $G_{i+1}$ (under the canonical maps $X_{j} \rightarrow G_{i+1}, j=i+1, \ldots, n$, has a solution in $G_{i+1}$. The coordinate group of an NTQ system is called an NTQ group.

An NTQ system $S=1$ is called regular if each non-empty quadratic equation in $S_{i}$ is regular (see Definition 1.16).

We say that an NTQ system system $S(X)=1$ is in standard form if all quadratic equations in item 1) of the definition are in standard form. Clearly, every NTQ system $S(X)=1$ is rationally equivalent to a unique NTQ system in standard form, namely, there exists an automorphism $\phi \in A u t F[X]$ such that $S^{\phi}=1$ is a standard NTQ system. Let $S=1$ be a standard NTQ system. Denote by $S_{R}$ the set of all regular quadratic equations in $S=1$

Let $n_{1} \geq n_{2} \ldots \geq n_{k}$ be the sequence of sizes of equations from $S_{R}$ in the decreasing order. Then the tuple $\operatorname{rsize}(S)=\left(n_{1}, \ldots, n_{k}\right)$ is called the regular size of the system $S=1$. We compare sizes of systems lexicographically from the left.

2.14. Rational equivalence. Recall, that two systems of equations $S(X)=$ 1 and $T(Y)=1$ with coefficients from $F$ are rationally equivalent if there are polynomial maps $Y=P_{X}(X)$ and $X=P_{Y}(Y)$ such that the restriction $P_{S}$ of the map $P_{X}: F^{|X|} \rightarrow F^{|Y|}$ onto the algebraic set $V_{F}(S)$ gives a bijection $P_{S}: V_{F}(S) \rightarrow$ $V_{F}(T)$, and the restriction $P_{T}$ of the map $P_{Y}$ onto the algebraic set $V_{F}(T)$ gives the inverse of $P_{S}$. Sometimes we refer to the maps $P_{X}$ and $P_{Y}$ as to change of coordinates. It was shown in (see 4) that systems $S(X)=1$ and $T(Y)=1$ are rationally equivalent if and only if their coordinate groups $F_{R(S)}$ and $F_{R(T)}$ are isomorphic as $F$-groups. Observe that if $f: F_{R(S)} \rightarrow F_{R(T)}$ is an $F$-isomorphism, then $X=f(X)$ and $Y=f^{-1}(Y)$ are corresponding change of coordinates for the systems $S(X)=1$ and $T(Y)=1$.

Definition 2.18. A system $S(X)=1$ with coefficients in a group $G$ splits if there exist a nontrivial partition of $X$ into $k>1$ disjoint subsets $X=X_{1} \cup \ldots \cup X_{k}$, and elements $S_{i}\left(X_{i}\right) \in G\left[X_{i}\right]$ such that $S(X)=1$ is the union of the systems $S_{i}\left(X_{i}\right)=1, i=1, \ldots, k$. 
We say that a system $S(X)=1$ with coefficients in $G$ splits up to the rational equivalence or rationally splits if some system $T(Y)=1$ which is rationally equivalent to $S(X)=1$ splits.

Notice that $S(X)=1$ rationally splits if and only if its coordinate group $G_{R(S)}$ is a nontrivial free product of the coordinate groups $G_{R\left(S_{i}\right)}$ with the group of constants $G$ amalgamated. In the case when the systems $S_{i}\left(X_{i}\right)=1$ are coefficient free for $i \neq 1$, one has $G_{R(S)} \simeq G_{1} * \cdots * G_{n}$.

2.15. Canonical automorphisms. Let $G=A *_{C} B$ be an elementary abelian splitting of $G$. For $c \in C$ we define an automorphism $\phi_{c}: G \rightarrow G$ such that $\phi_{c}(a)=a$ for $a \in A$ and $\phi_{c}(b)=b^{c}=c^{-1} b c$ for $b \in B$.

If $G=A *_{C}=\left\langle A, t \mid c^{t}=c^{\prime}, c \in C\right\rangle$ then for $c \in C$ define $\phi_{c}: G \rightarrow G$ such that $\phi_{c}(a)=a$ for $a \in A$ and $\phi_{c}(t)=c t$.

We call $\phi_{c}$ a Dehn twist obtained from the corresponding elementary abelian splitting of $G$. If $G$ is an $F$-group, where $F$ is a subgroup of one of the factors $A$ or $B$, then Dehn twists that fix elements of the free group $F \leq A$ are called canonical Dehn twists. Similarly, one can define canonical Dehn twists with respect to an arbitrary fixed subgroup $K$ of $G$.

Definition 2.19. Let $D \in \mathcal{D}(G)\left[D \in \mathcal{D}_{F}(G]\right.$ be an abelian splitting of a group $G$ and $G_{v}$ be either a $Q H$ or an abelian vertex of $D$. Then an automorphism $\psi \in \operatorname{Aut}(G)$ is called a canonical automorphism corresponding to the vertex $G_{v}$ if $\psi$ satisfies the following conditions:

1) $\psi$ fixes element-wise all other vertex groups in $D$ (hence fixes all the edge groups);

2) if $G_{v}$ is a $Q H$-vertex in $D$, then $\psi$ is a Dehn twist [canonical Dehn twists] corresponding to some essential $\mathbb{Z}$-splitting of $G$ along a cyclic subgroup of $G_{v}$;

3) if $G_{v}$ is an abelian subgroup then $\psi$ acts as an automorphism on $G_{v}$ which fixes all the edge subgroups of $G_{v}$.

Definition 2.20. Let $D \in \mathcal{D}(G)\left[D \in \mathcal{D}_{F}(G)\right]$ be an abelian splitting of a group $G$ and $e$ an edge in $D$. Then an automorphism $\psi \in \operatorname{Aut}(G)$ is called a canonical automorphism corresponding to the edge $e$ if $\psi$ is a Dehn twist [canonical Dehn twist] of $G$ with respect to the elementary splitting of $G$ along the edge $e$ which is induced from $D$.

Definition 2.21. Let $D \in \mathcal{D}(G)\left[D \in \mathcal{D}_{F}(G]\right.$ be an abelian splitting of a group [F-group] $G$. Then the canonical group of automorphisms $A_{D}=A_{D}(G)$ of $G$ with respect to $D$ is the subgroup of $\operatorname{Aut}(G)$ generated by all canonical automorphisms of $G$ corresponding to all edges, all $Q H$ vertices, and all abelian vertices of $D$.

2.16. Canonical automorphisms of QH-subgroups (orientable case). In this section we discuss some canonical automorphisms of QH-subgroups. Let $G \in \mathcal{F}$.

Let the relation corresponding to a $\mathrm{QH}$ subgroup $Q$ in some decomposition of $G$ be

$$
S=\prod_{i=1}^{n}\left[x_{i}, y_{i}\right] \prod_{j=1}^{m} c_{j}^{z_{j}} d^{-1}=1 .
$$

We define the basic sequence

$$
\Gamma=\left(\gamma_{1}, \gamma_{2}, \ldots, \gamma_{K(m, n)}\right)
$$


of canonical automorphisms of the group $G$ corresponding to $Q$. Let $X=\left\{x_{1}, y_{1}, \ldots, x_{n}, y_{n}, z_{1}, \ldots, z_{m}\right\}$. We assume that each $\gamma \in \Gamma$ acts identically on all the generators from $X$ that are not mentioned in the description of $\gamma$.

Let $m \geq 1, n=0$. In this case $K(m, 0)=m-1$. Put

$\gamma_{i}: z_{i} \rightarrow z_{i}\left(c_{i}^{z_{i}} c_{i+1}^{z_{i+1}}\right), \quad z_{i+1} \rightarrow z_{i+1}\left(c_{i}^{z_{i}} c_{i+1}^{z_{i+1}}\right), \quad$ for $i=1, \ldots, m-1$.

Notice that this is a Dehn twists corresponding to the splitting of $G$ as an HNN extension with the cyclic edge group $\left\langle p_{i} p_{i+1}\right\rangle$ belonging to $Q$.

Let $m=0, n \geq 1$. In this case $K(0, n)=4 n-1$. Put

$\gamma_{4 i-3}: y_{i} \rightarrow x_{i} y_{i}, \quad$ for $i=1, \ldots, n$ (this is a Dehn twist corresponding to the splitting of $G$ as an HNN extension with the edge group $\left\langle x_{i}\right\rangle$ and stable letter $y_{i}$ );

$\gamma_{4 i-2}: x_{i} \rightarrow y_{i} x_{i}, \quad$ for $i=1, \ldots, n$

$\gamma_{4 i-1}: y_{i} \rightarrow x_{i} y_{i}, \quad$ for $i=1, \ldots, n$;

$\left(y_{i} x_{i+1}^{-1}\right)^{-1} y_{i+1}, \quad x$

for $i=1, \ldots, n-1$.

Let $m \geq 1, n \geq 1$. In this case $K(m, n)=m+4 n-1$. Put

$$
\begin{aligned}
& \gamma_{i}: z_{i} \rightarrow z_{i}\left(c_{i}^{z_{i}} c_{i+1}^{z_{i+1}}\right), \quad z_{i+1} \rightarrow z_{i+1}\left(c_{i}^{z_{i}} c_{i+1}^{z_{i+1}}\right), \quad \text { for } i=1, \ldots, m-1 ; \\
& \gamma_{m}: z_{m} \rightarrow z_{m}\left(c_{m}^{z_{m}} x_{1}^{-1}\right), \quad x_{1} \rightarrow x_{1}^{c_{m}^{z_{m}} x_{1}^{-1}}, \quad y_{1} \rightarrow\left(c_{m}^{z_{m}} x_{1}^{-1}\right)^{-1} y_{1} ; \\
& \gamma_{m+4 i-3}: y_{i} \rightarrow x_{i} y_{i}, \quad \text { for } i=1, \ldots, n ; \\
& \gamma_{m+4 i-2}: x_{i} \rightarrow y_{i} x_{i}, \quad \text { for } i=1, \ldots, n ; \\
& \gamma_{m+4 i-1}: y_{i} \rightarrow x_{i} y_{i}, \quad \text { for } i=1, \ldots, n ; \\
& \gamma_{m+4 i}: x_{i} \rightarrow\left(y_{i} x_{i+1}^{-1}\right)^{-1} x_{i}, \quad y_{i} \rightarrow y_{i}^{y_{i} x_{i+1}^{-1}}, \quad x_{i+1} \rightarrow x_{i+1}^{y_{i} x_{i+1}^{-1}}, \quad y_{i+1} \rightarrow \\
& \left(y_{i} x_{i+1}^{-1}\right)^{-1} y_{i+1},
\end{aligned}
$$

for $i=1, \ldots, n-1$ (this is a Dehn twist corresponding to the splitting of $G$ with an edge group $\left\langle y_{i} x_{i+1}^{-1}\right\rangle$ belonging to $Q$ ).

Observe, that in the case $m \neq 0, n \neq 0$ the basic sequence of automorphisms $\Gamma$ contains the basic automorphisms from the other two cases. This allows us, without loss of generality, to formulate the results below only for the case $K(m, n)=$ $m+4 n-1$. Obvious adjustments provide the proper argument in the other cases.

The following lemma describes the action of powers of basic automorphisms from $\Gamma$ on $X$. The proof is obvious, and we omit it.

Lemma 2.22. Let $\Gamma=\left(\gamma_{1}, \ldots, \gamma_{m+4 n-1}\right)$ be the basic sequence of automorphisms and $p$ be a positive integer. Then the following hold:

$$
\begin{aligned}
& \gamma_{i}^{p}: z_{i} \rightarrow z_{i}\left(c_{i}^{z_{i}} c_{i+1}^{z_{i+1}}\right)^{p}, \quad z_{i+1} \rightarrow z_{i+1}\left(c_{i}^{z_{i}} c_{i+1}^{z_{i+1}}\right)^{p}, \quad \text { for } i=1, \ldots, m-1 ; \\
& \gamma_{m}^{p}: z_{m} \rightarrow z_{m}\left(c_{m}^{z_{m}} x_{1}^{-1}\right)^{p}, \quad x_{1} \rightarrow x_{1}^{\left(c_{m}^{z} x_{1}^{-1}\right)^{p}}, \quad y_{1} \rightarrow\left(c_{m}^{z_{m}} x_{1}^{-1}\right)^{-p} y_{1} ; \\
& \gamma_{m+4 i-3}^{p}: y_{i} \rightarrow x_{i}^{p} y_{i}, \quad \text { for } i=1, \ldots, n ; \\
& \gamma_{m+4 i-2}^{p}: x_{i} \rightarrow y_{i}^{p} x_{i}, \quad \text { for } i=1, \ldots, n ; \\
& \gamma_{m+4 i-1}^{p}: y_{i} \rightarrow x_{i}^{p} y_{i}, \quad \text { for } i=1, \ldots, n ;
\end{aligned}
$$


$\gamma_{m+4 i}^{p} \quad: \quad x_{i} \rightarrow\left(y_{i} x_{i+1}^{-1}\right)^{-p} x_{i}, y_{i} \rightarrow y_{i}^{\left(y_{i} x_{i+1}^{-1}\right)^{p}}, x_{i+1} \rightarrow x_{i+1}^{\left(y_{i} x_{i+1}^{-1}\right)^{p}}, \quad y_{i+1} \rightarrow$
$\left(y_{i} x_{i+1}^{-1}\right)^{-p} y_{i+1}$,
$\quad$ for $i=1, \ldots, n-1$.

Now we introduce vector notations for automorphisms of particular type.

Let $\mathbb{N}$ be the set of all positive integers and $\mathbb{N}^{k}$ the set of all $k$-tuples of elements from $\mathbb{N}$. For $s \in \mathbb{N}$ and $p \in \mathbb{N}^{k}$ we say that the tuple $p$ is $s$-large if every coordinate of $p$ is greater then $s$. Similarly, a subset $P \subset \mathbb{N}^{k}$ is $s$-large if every tuple in $P$ is $s$-large. We say that the set $P$ is unbounded if for any $s \in \mathbb{N}$ there exists an $s$-large tuple in $P$.

Let $\delta=\left(\delta_{1}, \ldots, \delta_{k}\right)$ be a sequence of automorphisms of the group $F_{R(S)}$, and $p=\left(p_{1}, \ldots, p_{k}\right) \in \mathbb{N}^{k}$. Then by $\delta^{p}$ we denote the following automorphism of $F_{R(S)}$ :

$$
\delta^{p}=\delta_{1}^{p_{1}} \ldots \delta_{k}^{p_{k}} \text {. }
$$

Notation Let $\Gamma=\left(\gamma_{1}, \ldots, \gamma_{K}\right)$ be the basic sequence of automorphisms for $S=$ 1. Denote by $\Gamma_{\infty}$ the infinite periodic sequence with period $\Gamma$, i.e., $\Gamma_{\infty}=\left\{\gamma_{i}\right\}_{i \geq 1}$ with $\gamma_{i+K}=\gamma_{i}$. For $j \in \mathbb{N}$ denote by $\Gamma_{j}$ the initial segment of $\Gamma_{\infty}$ of the length $j$. Then for a given $j$ and $p \in \mathbb{N}^{j}$ put

$$
\phi_{j, p}=\overleftarrow{\Gamma}_{j}^{\overleftarrow{p}}=\gamma_{j}^{p_{j}} \gamma_{j-1}^{p_{j-1}} \ldots \gamma_{1}^{p_{1}}
$$

Sometimes we omit $p$ from $\phi_{j, p}$ and write simply $\phi_{j}$.

2.17. Canonical automorphisms of QH subgroups (non-orientable case). Similarly we introduce the notion of the basic sequence of automorphisms for a nonorientable QH subgroup. It is more convenient to consider a non-orientable relation in the form

or

$$
S=\prod_{i=1}^{m} z_{i}^{-1} c_{i} z_{i} \prod_{i=1}^{n}\left[x_{i}, y_{i}\right] x_{n+1}^{2}=c_{1} \ldots c_{m} \prod_{i=1}^{n}\left[a_{i}, b_{i}\right] a_{n+1}^{2},
$$

$$
S=\prod_{i=1}^{m} z_{i}^{-1} c_{i} z_{i} \prod_{i=1}^{n}\left[x_{i}, y_{i}\right] x_{n+1}^{2} x_{n+2}^{2}=c_{1} \ldots c_{m} \prod_{i=1}^{n}\left[a_{i}, b_{i}\right] a_{n+1}^{2} a_{n+2}^{2} .
$$

Without loss of generality we consider equation (11). We define a basic sequence

$$
\Gamma=\left(\gamma_{1}, \gamma_{2}, \ldots, \gamma_{K(m, n)}\right)
$$

of automorphisms of $F_{R(S)}$. We assume that each $\gamma \in \Gamma$ acts identically on all the generators from $X$ that are not mentioned in the description of $\gamma$. Automorphisms $\gamma_{i}, i=1, \ldots, m+4 n-1$ are the same as in the orientable case.

Let $n=0$. In this case $K=K(m, 0)=m+2$. Put

$$
\begin{aligned}
& \gamma_{m}: z_{m} \rightarrow z_{m}\left(c_{m}^{z_{m}} x_{1}^{2}\right), \quad x_{1} \rightarrow x_{1}^{\left(c_{m}^{z_{m}} x_{1}^{2}\right)} \\
& \gamma_{m+1}: x_{1} \rightarrow x_{1}\left(x_{1} x_{2}\right), \quad x_{2} \rightarrow\left(x_{1} x_{2}\right)^{-1} x_{2} ; \\
& \gamma_{m+2}: x_{1} \rightarrow x_{1}^{\left(x_{1}^{2} x_{2}^{2}\right)}, \quad x_{2} \rightarrow x_{2}^{\left(x_{1}^{2} x_{2}^{2}\right)} .
\end{aligned}
$$

Let $n \geq 1$. In this case $K=K(m, n)=m+4 n+2$. Put

$$
\gamma_{m+4 n}: x_{n} \rightarrow\left(y_{n} x_{n+1}^{2}\right)^{-1} x_{n}, \quad y_{n} \rightarrow y_{n}^{\left(y_{n} x_{n+1}^{2}\right)}, \quad x_{n+1} \rightarrow x_{n+1}^{\left(y_{n} x_{n+1}^{2}\right)} ;
$$


$\gamma_{m+4 n+1}: x_{n+1} \rightarrow x_{n+1}\left(x_{n+1} x_{n+2}\right), \quad x_{n+2} \rightarrow\left(x_{n+1} x_{n+2}\right)^{-1} x_{n+2}$;

$\gamma_{m+4 n+2}: x_{n+1} \rightarrow x_{n+1}^{\left(x_{n+1}^{2} x_{n+2}^{2}\right)}, \quad x_{n+2} \rightarrow x_{n+2}^{\left(x_{n+1}^{2} x_{n+2}^{2}\right)}$.

A family of automorphisms

$$
\Gamma_{P}=\left\{\phi_{j, p}, j \in N_{0}, p \in P\right\}
$$

is called positive unbounded if $P$ and $N_{0} \subseteq \mathbb{N}$ are unbounded. A family of homomorphisms $\Gamma_{P} \beta$ from $F_{R(S)}$ onto $F$, where $\beta$ is a solution in general position, and $\Gamma_{P}$ is positive unbounded, is a generic family (see Definition 12.8 below).

2.18. Minimal solutions and maximal standard quotients. Let $G$ and $K$ be $H$-groups and $A \leq A u t_{H}(G)$ a group of $H$-automorphisms of $G$. Two $H$ homomorphisms $\phi$ and $\psi$ from $G$ into $K$ are called A-equivalent (symbolically, $\phi \sim_{A} \psi$ ) if there exists $\sigma \in A$ such that $\phi=\sigma \psi$ (i.e., $g^{\phi}=\left(g^{\sigma}\right)^{\psi}$ for $g \in G$ ). Obviously, $\sim_{A}$ is an equivalence relation on $\operatorname{Hom}_{H}(G, K)$.

Let $D$ be a fixed abelian splitting of $G$ and $A_{D}=A_{D}(G)$.

Definition 2.23. Let $S(X, A)=1$ be a system of equations over $F=F(A)$, $G=F_{R(S)}$, and $D \in \mathcal{D}(G)$. Suppose that $\phi_{1}, \phi_{2}: F_{R(S)} \rightarrow F(A \cup Y)$ are solutions of $S=1$ in a free group $\bar{F}=F(A \cup Y)$. We write $\phi_{1}<_{D} \phi_{2}$ if there exists an automorphism $\sigma \in A_{D}$ and an endomorphism $\pi \in \operatorname{Hom}_{F}(\bar{F}, \bar{F})$ such that $\phi_{2}=\sigma^{-1} \phi_{1} \pi$ (see Fig. 1) and

$$
\sum_{x \in X}\left|x^{\phi_{1}}\right|<\sum_{x \in X}\left|x^{\phi_{2}}\right|
$$

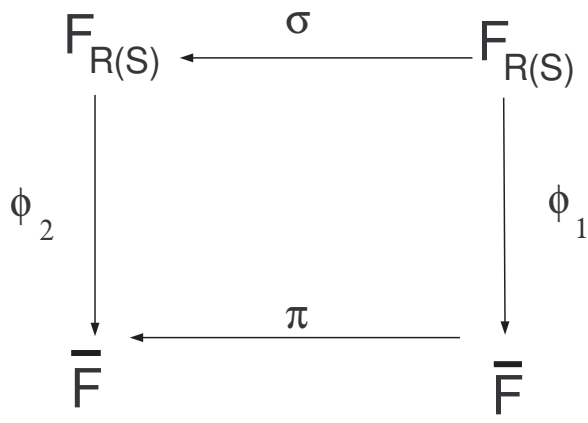

FiguRE 1. $\phi_{1}<_{D} \phi_{2}$

Notice that if $Y=\emptyset$ then $\phi_{1}<\phi_{2}$ if and only if $\phi_{1} \sim_{A_{D}} \phi_{2}$ and $\sum_{x \in X}\left|x^{\phi_{1}}\right|<$ $\sum_{x \in X}\left|x^{\phi_{2}}\right|$. This provides a way to chose representatives in $\sim_{A_{D}}$-equivalence classes of automorphisms. A solution $\phi: G \rightarrow F$ is called minimal if $\phi$ is $<_{D}$-minimal in its $\sim_{A_{D}}$-equivalence class.

Let $R_{D}$ be the intersection of the kernels of all minimal homomorphisms from $\operatorname{Hom}_{F}(G, F)$. Then $G / R_{D}$ is called the maximal standard quotient of $G$ relative to $D$. 
Lemma 2.24. Let $D$ be a fixed abelian splitting of $G$. Then every $F$-homomorphism from $G$ onto $F$ can be presented as composition of a canonical (relative to D) automorphism of $G$, the canonical epimorphism $G \rightarrow G / R_{D}$, and an $F$-homomorphism from $G / R_{D}$ onto $F$.

Lemma 2.25. Let $G$ be a freely indecomposable $F$-group in $\mathcal{F}$, and $D$ be an abelian JSJ decomposition of $G$. Then $A_{D}$ is a direct product of abelian groups generated by canonical Dehn twists corresponding to edges of $D$ between non-QH non-abelian subgroups, and groups of canonical automorphisms corresponding to $M Q H$ and abelian vertex groups.

Proof. Consider two edges $e_{1}, e_{2}$ of $D$ such that $e_{1} \in T$ and $e_{2} \notin T$. Collapsing all the other edges we obtain $G=\left\langle H *\langle C\rangle, K, t \mid A^{t}=B\right\rangle$, where $F \leq H, A \leq H, B \leq$ $K$. We write down the action of canonical Dehn twists corresponding to the edges of this decomposition on the generators of $G$. Let $c \in C, b \in B$. Then

$$
\begin{gathered}
x^{\phi_{c}}=x, x \in H, \quad y^{\phi_{c}}=y^{c}, y \in K, \quad t^{\phi_{c}}=t c, \\
x^{\phi_{b}}=x, x \in H, \quad y^{\phi_{b}}=y, y \in K, \quad t^{\phi_{b}}=t b .
\end{gathered}
$$

In this case, $\phi_{c} \phi_{b}=\phi_{b} \phi_{c}$

All the other possible cases can be verified similarly.

2.19. Sufficient splittings. Let $H \leq G$. A family of $H$-homomorphisms

$$
\Psi=\{\psi: G \rightarrow H\}
$$

is called separating if for any nontrivial $g \in G$ there exists $\psi \in \Psi$ such that $\psi(g) \neq 1$ in $H$.

Definition 2.26. Let $G$ be a group, $\mathcal{K}=\left\{K_{1}, \ldots, K_{n}\right\}$ be a set of subgroups of $G$ and $F$ is a fixed subgroup of $K_{1}$. We say that there is a sufficient splitting of $G$ modulo $\mathcal{K}$ if one of the following holds:

1) $G$ is freely decomposable modulo $\mathcal{K}$,

2) there exists a reduced $\mathcal{K}$-compatible free decomposition of $G$ in which at least one factor $G_{j}$ has an abelian splitting $D$ such that all subgroups from $\mathcal{K}_{j}$ are elliptic in $D$ and such that $\operatorname{Rep}_{\mathcal{K}_{j}}\left(G_{j}, D\right)$ is not a separating family of homomorphisms ( $F$-homomorphisms, if $j=1$ ) from $G_{j}$ into $F$.

In the case of a freely indecomposable group $G, D$ is a sufficient splitting of $G$ if and only if the standard maximal quotient $G / R_{D}$ is a proper quotient of $G$.

2.20. Maximal standard fully residually free quotients. Let $G$ be a group with a finite generating set $X$ and $D$ an abelian splitting of $G$. Denote by $G / R_{D}=\langle X \mid S\rangle$ a presentation of the maximal standard quotient $G / R_{D}$ relative to $X$. Then the coordinate group $F_{R(S)}$ is a quotient of $G / R_{D}$. If $S_{1}=1, \ldots, S_{k}=1$ are finite systems that determine the irreducible components of the algebraic set $V_{F}(S)$ then (see $\underline{4}$ )

$$
R(S)=R\left(S_{1}\right) \cap \ldots \cap R\left(S_{k}\right)
$$

and the radicals $R\left(S_{i}\right)$ are uniquely defined (up to reordering). Notice that the coordinate groups $F_{R\left(S_{i}\right)}$ are fully residually free, they are called the standard maximal fully residually free quotients of $G$ relative to $D$.

Lemma 2.27. The restriction of the canonical epimorphism $\pi: G \rightarrow G / R_{D}$ onto a rigid subgroup of $D$ and onto a subgroup of the abelian vertex group generated by the images of edge groups, is a monomorphism. 
Proof. Let $H$ be a rigid subgroups of $D$. Canonical automorphisms corresponding to $D$ act on $H$ as conjugation. The set of all representatives of the equivalence classes of solutions $H \rightarrow F$ with respect to conjugation is a discriminating set for $H$. Similar argument works for a subgroup of the abelian vertex group generated by the images of edge groups.

COROLLARY 2.28. There exists a standard maximal fully residually free quotient $K$ of $G$ such that all the restrictions of the canonical epimorphism $G \rightarrow K$ onto rigid subgroups of $D$, onto edge subgroups in $D$, and onto the subgroups of abelian vertex groups $A$ generated by the images of all the edge groups of edges adjacent to $A$, are monomorphisms.

Proof. Let $K_{1}, \ldots, K_{s}$ be all maximal standard fully residually free quotients of $G$. Let $\phi_{i}: G / R_{D} \rightarrow K_{i}$ be the canonical epimorphism. Suppose that for each $i$ there exists an element $u_{i}$ in some rigid subgroup of $D$ or in a subgroup of the abelian vertex group generated by the images of edge groups such that $\phi_{i}\left(u_{i}\right)=1$. Since $G$ is fully residually free, and for any homomorphism from $G$ to $F$ the image of $u_{i}$ is a conjugate of $\phi_{i}\left(u_{i}\right)=1$, there is some $i$ such that each homomorphism from $G$ to $F$ satisfies the equation $u_{i}=1$.

\section{Algorithms over fully residually free groups}

3.1. Algorithms for equations and coordinate groups. In this section we collect some results on algorithmic problems concerning equations over free groups and their coordinate groups. We assume below that a coordinate group $G$ is given by a finite system of equations $S(X)=1$ over $F$ in such a way that $G=F_{R(S)}$.

THEOREM 3.1. The following statements are true:

(1) There is an algorithm which for a given finite system of equations $S(X)=$ 1 over $F$ and a given group word $w(X)$ in $X \cup A$ determines whether $w(X)$ is equal to 1 in $F_{R(S)}$ or not;

(2) There is an algorithm which for a given finite systems of equations $S(X)=$ $1, T(X)=1$ over $F$ decides whether or not $R(S)=R(T)$.

Proof. (1) Let $S(X)=1$ be a finite system of equations over $F$ and $w$ a group word in the alphabet $X \cup A$. Then the universal sentence

$$
\Phi_{S, w}=\forall X(S(X)=1 \rightarrow w(X)=1)
$$

is true in the free group $F$ if and only if $w \in R(S)$. Since the universal theory $T h_{\forall}(F)$ is decidable [52] one can effectively check whether or not $\Phi_{S, w} \in T h_{\forall}(F)$. This proves (1). Now (2) follows immediately from (1).

THEOREM 3.2. ( $\mathbf{3 6}$, see also Theorem 8.2 below.) There is an algorithm which for a given system of equations $S(X)=1$ over $F$ finds finitely many NTQ-systems $Q_{1}=1, \ldots, Q_{n}=1$ over $F$ and $F$-homomorphisms $\phi_{i}: F_{R(S)} \rightarrow F_{R\left(Q_{i}\right)}$ such that any $F$-homomorphism $\lambda: F_{R(S)} \rightarrow F$ factors through one of the homomorphisms $\phi_{i}, i=1, \ldots, n$.

COROLlary 3.3. There is an algorithms which for a given finite system of equations $S(X)=1$ over $F$ finds finitely many groups $G_{1}, \ldots, G_{n} \in \mathcal{F}$ (given by finite presentations in generators $X \cup A)$ and epimorphisms $\phi_{i}: F_{R(S)} \rightarrow G_{i}$ such that any homomorphism $\phi: F_{R(S)} \rightarrow F$ factors through one of the epimorphisms $\phi_{1}, \ldots, \phi_{n}$. 
Proof. The result follows from Theorem 3.2 and Theorem 3.20

THEOREM 3.4. ( 35 ) Given an NTQ system $Q$ over $F$ one can effectively find an embedding $\phi: F_{R(Q)} \rightarrow F^{\mathbb{Z}[t]}$.

THEOREM 3.5. Given a finite system of equations $S=1$ over $F$ and an $F$ homomorphism $\phi: F_{R(S)} \rightarrow H$ of $F_{R(S)}$ into an NTQ F-group $H$ one can effectively decide whether $\phi$ is a monomorphism or not.

Proof. Let $G=F_{R(S)}$ for a finite system $S=1$ over $F$ and $H$ an NTQ $F$-group. Suppose $\phi: F_{R(S)} \rightarrow H$ is a homomorphism given by the set of images $X^{\phi} \subseteq H$. By Theorem 3.20 one can effectively find a finite set, say $T$, of defining relations of the subgroup $G^{\phi}=\left\langle X^{\phi}\right\rangle \leq H$ with respect to the generating set $X^{\phi}$. It follows that $R(S) \leq R(T)$. Clearly, $\phi$ is a monomorphism if and only if $R(S)=R(T)$. The latter can be checked effectively by Theorem 3.1

THEOREM 3.6. Given a finite irreducible system of equations $S=1$ over $F$ one can effectively find an NTQ system $Q$ over $F$ and an embedding $\phi: F_{R(S)} \rightarrow F_{R(Q)}$.

Proof. By Theorem 3.2 one can effectively find finitely many NTQ systems $Q_{1}, \ldots, Q_{n}$ over $F$ and $F$-homomorphisms $\phi_{i}: F_{R(S)} \rightarrow F_{R\left(Q_{i}\right)}$ such that any $F$-homomorphism $\lambda: F_{R(S)} \rightarrow F$ factors through one of the homomorphisms $\phi_{i}, i=1, \ldots, n$. It is known (see 35, 36, 4]) that in this case at least one of the homomorphisms $\phi_{i}$ is monic. Now by Theorem 3.5 one can effectively check which homomorphisms among $\phi_{1}, \ldots, \phi_{n}$ are monic. This proves the theorem.

THEOREM 3.7. There is an algorithm which for a given finite irreducible system of equations $S(X)=1$ over $F$ finds a finite representation of the coordinate group $F_{R(S)}$ with respect to the generating set $X \cup A$.

Proof. By Theorem 3.6 one can effectively find an NTQ system $Q$ over $F$ and an embedding $\phi: F_{R(S)} \rightarrow F_{R(Q)}$. It follows that one can effectively find the finite generating set $X^{\phi} \cup A$ of the subgroup $F_{R(S)}^{\phi}$ of the group $F_{R(Q)}$. Now, since the group $F_{R(Q)}$ belongs to $\mathcal{F}$ the result follows immediately from Theorem 3.20

COROLlary 3.8. For every finite irreducible system of equations $S=1$ one can effectively find the radical $R(S)$ by specifying a finite set of generators of $R(S)$ as a normal subgroup.

THEOREM 3.9. (35) There is an algorithm which for a given finite system of equations $S(X)=1$ over $F$ finds its irreducible components.

ProOF. We give here another, more direct, proof of this result. By Corollary 3.3 one can effectively find finitely many groups $G_{1}, \ldots, G_{n} \in \mathcal{F}$, given by finite presentations $\left\langle X \cup A \mid T_{i}\right\rangle$, and epimorphisms $\phi_{i}: F_{R(S)} \rightarrow G_{i}$ such that any homomorphism $\phi: G F_{R(S)} \rightarrow F$ factors through one of the epimorphisms $\phi_{1}, \ldots, p h i_{n}$. It is not hard to see that the systems $T_{i}=1$ are irreducible over $F$ and

$$
V_{F}(S)=V_{F}\left(T_{1}\right) \cup \ldots V_{F}\left(T_{n}\right)
$$

Now by Theorem 3.1 one can check effectively whether or not $V_{F}\left(T_{i}\right)=V_{F}\left(T_{j}\right)$ or $V_{F}\left(T_{i}\right)=V_{F}(S)$, thus producing all irreducible components of $V_{F}(S)$. 
3.2. Algorithms for finitely generated fully residually free groups. In this section we collect some results on algorithmic problems for finitely generated fully residually free groups from $36,40,46,57$. We assume below that groups from $\mathcal{F}$ are given by finite presentations. Notice that if $\langle X \mid S\rangle$ is a finite presentation of a group $G \in \mathcal{F}$ then $S(X)=1$ can be viewed as a finite irreducible coefficient-free system of equations over $F$ and $R(S)=n c l(S)$ where the radical $R(S)$ is taken in the free group $F(X)$ (without coefficients from $F$ ). This allows one to apply the algorithmic results from the Section 3.1 to groups from $\mathcal{F}$.

TheOREM 3.10. The word problem is decidable in groups from $\mathcal{F}$.

Proof. Let $G \in \mathcal{F}$ and $g \in G$. Since $G$ is finitely presented one can effectively enumerate all consequences of relators of $G$, so if $g=1$ then $g$ will occur in this enumeration. On the other hand, one can effectively enumerate all homomorphisms $\phi_{1}, \phi_{2}, \ldots$, from $G$ into a given free group $F$ (say of rank 2). If $g \neq 1$ then, since $G$ is residually free, there exists $\phi_{i}$ such that $\phi_{i}(g) \neq 1$, which can be verified effectively (by trying one by one all the images $\phi_{1}(g), \phi_{2}(g), \ldots$, ). This shows that the word problem is decidable in $G$, as required.

Theorem 3.11. 46 The conjugacy problem is decidable in groups from $\mathcal{F}$.

Notice, that Theorem 3.11 also follows from [12, because finitely generated fully residually free groups are relatively hyperbolic $[\mathbf{2 0}]$.

TheOREM 3.12. [57. The membership problem is decidable in groups from $\mathcal{F}$. Namely, there exists an algorithm which for a group $G \in \mathcal{F}$ given by a finite presentation $\langle X \mid R\rangle$ and a finite tuple of words $h_{1}(X), \ldots, h_{k}(X), w(X)$ in the alphabet $X^{ \pm 1}$ decides whether or not the element $w(X)$ belongs to the subgroup $\left\langle h_{1}(X), \ldots, h_{k}(X)\right\rangle$.

Using an analog of Stallings' foldings introduced in [53 for finitely generated subgroups of $F^{\mathbb{Z}[t]}$, one can obtain the following results.

TheOREM 3.13. [46] Let $G \in \mathcal{F}$ and $H$ and $K$ finitely generated subgroups of $G$ given by finite generating sets. Then $H \cap K$ is finitely generated, and one can effectively find a finite set of generators of $H \cap K$.

TheOREM 3.14. 46 Let $G \in \mathcal{F}$ and $H$ and $K$ finitely generated subgroups of $G$ given by finite generating sets. Then one can effectively find a finite family $\mathcal{J}_{G}(H, K)$ of non-trivial finitely generated subgroups of $G$ (given by finite generating sets), such that

(1) every $J \in \mathcal{J}_{G}(H, K)$ is of one of the following types

$$
H^{g_{1}} \cap K, \quad H^{g_{1}} \cap C_{K}\left(g_{2}\right),
$$

where $g_{1} \in G \backslash H, g_{2} \in K$, moreover $g_{1}, g_{2}$ can be found effectively;

(2) for any non-trivial intersection $H^{g} \cap K, g \in G \backslash H$ there exists $J \in$ $\mathcal{J}_{G}(H, K)$ and $f \in K$ such that

$$
H^{g} \cap K=J^{f},
$$

moreover $J$ and $f$ can be found effectively.

COROLlarY 3.15. Let $H, K$ be finitely generated subgroups of finitely generated fully residually free group $G$. Then one can effectively verify whether or not $K$ is conjugate into $H$, and if it is, then find a conjugator. 
COROLlary 3.16. Let $H, K$ be finitely generated subgroups of finitely generated fully residually free group $G$, let $H$ be abelian. Then one can effectively find a finite family $\mathcal{J}$ of non-trivial intersections $J=H^{g} \cap K \neq 1$ such that any non-trivial intersection $H^{g_{1}} \cap K$ has form $J^{k}$ for some $k \in K$ and $J \in \mathcal{J}$. One can effectively find the generators of the subgroups from $\mathcal{J}$.

Theorem 3.17. Given a group $G \in \mathcal{F}$, a splitting $D$ of $G$, and a finitely generated freely indecomposable subgroup $H$ of $G$ (given by a finite generating set $Y$ ) one can effectively find the splitting $D_{H}$ of $H$ induced from $D$. Moreover, one can describe all the vertex and edge groups, and homomorphisms, which occur in $D_{H}$, explicitly as words in generators $Y$.

Proof. Following Lemma 2.1 one can construct effectively the graph of groups for the subgroup $H$ using Theorem 3.14 Indeed, for every vertex $v \in X$ by Theorem 3.14 one can find effectively the complete finite family of non-trivial subgroups such that each intersection $H \cap G_{v}^{g}$ were $g$ runs over $G$ contains a conjugate of one of them in $H$. If all these intersections are trivial then by Lemma 2.1 the subgroup $H$ is cyclic. Otherwise there exists a vertex $v \in X$ and an element $g \in G$ such that the subgroup $H_{v}=H \cap G_{v}^{g}$ is non-trivial. We start building the graph of groups $\Gamma_{H}$ for $H$ induced from $G$ with the graph of groups $\Gamma_{1}$ consisting of the vertex $v$ and the subgroup $H_{v}$ associated with it. Now for every edge $e$ outgoing from $v$ in the graph $X$ we find by Corollary 3.16 the complete finite set (up to conjugation in $H_{v}$ ) of non-trivial intersections $H_{e}=H_{v} \cap G_{e}^{g}$ were $g$ runs over $G$. If there are no non-trivial intersections of this type then either $H_{v}=H$ or $H_{v}$ is a free factor of $H$. Since, by Theorem 3.12 the membership problem is decidable in $G$ one can effectively check whether $H=H_{v}$ or not. Suppose that $H_{e}=H_{v} \cap G_{e}^{g} \neq 1$ for some $g \in G$ and an edge $e$ outgoing from $v$. If $u$ is the terminal vertex of $e$ then $H_{u} \neq 1$ and we have reconstructed an edge in the graph $\Gamma_{H}$. Denote by $\Gamma_{2}$ the graph of groups obtained from $\Gamma_{1}$ by adding the edge $e$ and the vertex $u$ with the associated subgroups $H_{e}$ and $H_{u}$. If the fundamental group $\pi\left(\Gamma_{2}\right)$ is equal to $H$ then we are finished, otherwise we continue as above. In finitely many steps we will get a graph of groups $\Gamma_{k}$ such that $\pi\left(\Gamma_{k}\right)=H$. This proves the theorem.

The Theorem 3.17 allows one to prove an effective version of Theorem 1.7

THEOREM 3.18. Let $G$ be a finitely generated group which is given as a finite sequence

$$
F=G_{0} \leq G_{1} \leq \ldots \leq G_{n}=G
$$

of extensions of centralizers $G_{i+1}=G_{i}\left(u_{i}, t_{i}\right)$. Then given a finite set of elements $Y \subseteq G$ one can effectively construct the subgroup $\langle Y\rangle$ generated by $Y$ in $G$ from free groups by finitely many operations of the following type:

(1) free products;

(2) free products with amalgamation along cyclic subgroups with at least one of them being maximal;

(3) separated HNN extensions along cyclic subgroups with at least one of them being maximal;

(4) free extensions of centralizers;

in such a way that all groups and homomorphisms which occur during this process are given explicitly as words in generators $Y$. 
Proof. The result follows from Theorem 3.17 by induction on the length of the sequence (13).

COROLlaRY 3.19. There is an algorithm which for a given finitely generated fully residually free group $G$ determines whether $G$ is hyperbolic or not.

Proof. Let $G$ be a finitely generated fully residually free group. By Theorems 3.4 and 3.6 one can effectively embed $G$ into a group $G_{n}$ which is obtained from a free group $F$ by a finite sequence of extensions of centralizers as in 13 Now, by Theorem 3.18 one can effectively construct $G$ from $F$ by finitely many operations of the type (1) - (4). It is known (see, for example, 33) that in this event $G$ is hyperbolic if and only if no operations of the type (4) occurred in the construction of $G$. The latter can be checked algorithmically when the finite sequence of operations is given.

As a corollary of the Theorem 3.17 one can immediately obtain the following result.

THEOREM 3.20. There is an algorithm which for a given NTQ system $Q$ over $F$ and a finitely generated subgroup $H \leq F_{R(Q)}$, given by a finite generating set $Y$, finds a finite presentation for $H$ in the generators $Y$.

Proof. By Theorem 3.4 one can effectively embed $F_{R(Q)}$ into $F^{\mathbb{Z}[t]}$. It follows that one can effectively embed $F_{R(Q)}$ into a finitely generated group $H$ which is obtained from $F$ by a finite sequence of extensions of centralizer. Now the result follows from Theorem 3.18

THEOREM 3.21. There is an algorithm which for a given group $G \in \mathcal{F}$ and a finitely generated subgroup $H \leq G$ (given by a finite generating set $Y$ ), finds a finite presentation for $H$ in the generators $Y$.

Proof. Let $G=\langle X \mid S\rangle$ be a finite presentation of $G$. As we have mentioned above one can view the relations $S(X)=1$ as a coefficient-free finite irreducible system of equations over $F$ with the coordinate group $G$. By Theorem 3.6 one can effectively embed the group $G$ into the coordinate group $F_{R(Q)}$ of an NTQ system $Q$ over $F$. Now the result follows from Theorem 3.20 .

Observe also, that Theorem 3.20] can be derived from [32], Theorem 5.8.

THEOREM 3.22. There is an algorithm which for a given homomorphism $\phi$ : $G \rightarrow H$ between two groups from $\mathcal{F}$ decides whether or not:

(1) $\phi$ is an epimorphism;

(2) $\phi$ is a monomorphism;

(3) $\phi$ is an isomorphism.

Proof. Let $G, H \in \mathcal{F}$ and $\langle X \mid S\rangle$ a given finite presentation of $G$.

(1) Obviously, $\phi$ is onto if and only if $G^{\phi}=H$. Observe that $G^{\phi}$ is generated by a finite set $X^{\phi}$, so by Theorem 3.12 one can effectively verify whether $G^{\phi}=H$ or not, as required.

(2) By Theorem 3.20 one can effectively find a finite presentation, say $\langle X \mid T\rangle$, of the subgroup $G^{\phi}$ of $H$ with respect to the generating set $X^{\phi}$. Clearly, $\phi$ is monic if and only if $R(S)=R(T)$ which can be effectively verified by Theorem 3.1 This proves (2) and the theorem since (3) follows from (1) and (2). 
Theorem 3.23. 42] The Diophantine problem is decidable in groups from $\mathcal{F}$. Namely, there is an algorithm which for a given group $G \in \mathcal{F}$ and an equation $S=1$ over $G$ decides whether or not the equation $S=1$ has a solution in $G$ (and finds a solution if it exists).

\section{Generalized equations over free groups}

Makanin 51 introduced the concept of a generalized equation constructed for a finite system of equations in a free group $F=F(A)$. Geometrically a generalized equation consists of three kinds of objects: bases, boundaries and items. Roughly it is a long interval with marked division points. The marked division points are the boundaries. Subintervals between division points are items (we assign a variable to each item). Line segments below certain subintervals, beginning at some boundary and ending at some other boundary, are bases. Each base either corresponds to a letter from $A$ or has a double.

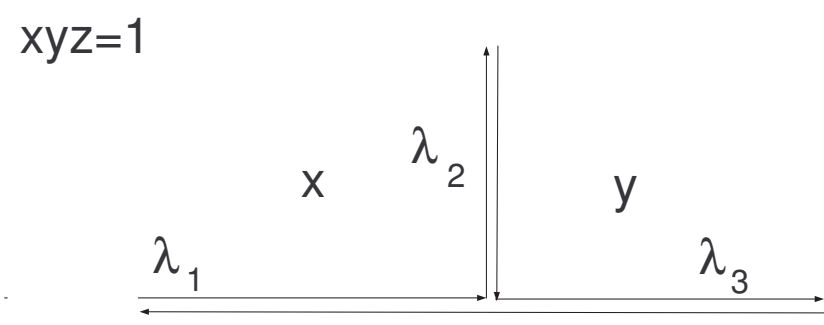

Z

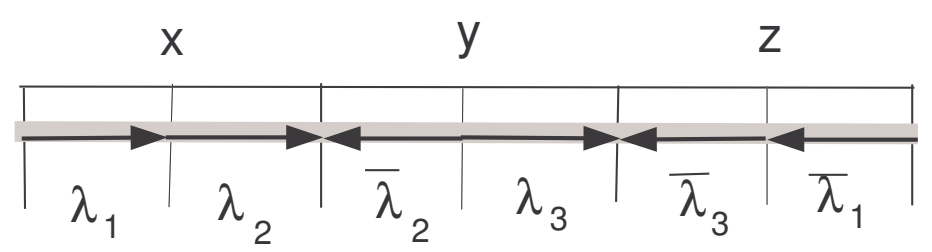

Figure 2. From the cancellation tree for the equation $x y z=1$ to the generalized equation $\left(x=\lambda_{1} \circ \lambda_{2}, y=\lambda_{2}^{-1} \circ \lambda_{3}, z=\lambda_{3}^{-1} \circ \lambda_{1}^{-1}\right)$.

This concept becomes crucial to our subsequent work and is difficult to understand. This is one of the main tools used to describe solution sets of systems of equations. In subsequent papers we will use it also to obtain effectively different splittings of groups. Before we give a formal definition we will try to motivate it with a simple example.

Suppose we have the simple equation $x y z=1$ in a free group. Suppose that we have a solution to this equation denoted by $x^{\phi}, y^{\phi}, z^{\phi}$ where is $\phi$ is a given homomorphism into a free group $F(A)$. Since $x^{\phi}, y^{\phi}, z^{\phi}$ are reduced words in the generators $A$ there must be complete cancellation. If we take a concatenation of the geodesic subpaths corresponding to $x^{\phi}, y^{\phi}$ and $z^{\phi}$ we obtain a path in the Cayley graph corresponding to this complete cancellation. This is called a cancellation tree 
(see Fig. 2). In the simplest situation $x=\lambda_{1} \circ \lambda_{2}, y=\lambda_{2}^{-1} \circ \lambda_{3}$ and $z=\lambda_{3}^{-1} \circ \lambda_{1}^{-1}$. The generalized equation would then be the following interval.

The boundaries would be the division points, the bases are the $\lambda^{\prime} s$ and the items in this simple case are also the $\lambda^{\prime} s$. In a more complicated equation where the variables $X, Y, Z$ appear more than one time this basic interval would be extended, Since the solution of any equation in a free group must involve complete cancellation this drawing of the interval is essentially the way one would solve such an equation. Our picture above depended on one fixed solution $\phi$. However for any equation there are only finitely many such cancellation trees and hence only finitely many generalized equations.

4.1. Generalized equations. Let $A=\left\{a_{1}, \ldots, a_{m}\right\}$ be a set of constants and $X=\left\{x_{1}, \ldots, x_{n}\right\}$ be a set of variables. Put $G=F(A)$ and $G[X]=G * F(X)$.

DeFinition 4.1. A combinatorial generalized equation $\Omega$ (with constants from $\left.A^{ \pm 1}\right)$ consists of the following objects:

1. A finite set of bases $B S=B S(\Omega)$. Every base is either a constant base or a variable base. Each constant base is associated with exactly one letter from $A^{ \pm 1}$. The set of variable bases $\mathcal{M}$ consists of $2 n$ elements $\mathcal{M}=\left\{\mu_{1}, \ldots, \mu_{2 n}\right\}$. The set $\mathcal{M}$ comes equipped with two functions: a function $\varepsilon: \mathcal{M} \rightarrow\{1,-1\}$ and an involution $\Delta: \mathcal{M} \rightarrow \mathcal{M}$ (i.e., $\Delta$ is a bijection such that $\Delta^{2}$ is an identity on $\mathcal{M}$ ). Bases $\mu$ and $\Delta(\mu)$ (or $\bar{\mu})$ are called dual bases. We denote variable bases by $\mu, \lambda, \ldots$

2. A set of boundaries $B D=B D(\Omega)$. $B D$ is a finite initial segment of the set of positive integers $B D=\{1,2, \ldots, \rho+1\}$. We use letters $i, j, \ldots$ for boundaries.

3. Two functions $\alpha: B S \rightarrow B D$ and $\beta: B S \rightarrow B D$. We call $\alpha(\mu)$ and $\beta(\mu)$ the initial and terminal boundaries of the base $\mu$ (or endpoints of $\mu$ ). These functions satisfy the following conditions: $\alpha(b)<\beta(b)$ for every base $b \in B S$; if $b$ is a constant base then $\beta(b)=\alpha(b)+1$.

4. A finite set of boundary connections $B C=B C(\Omega)$. A boundary connection is a triple $(i, \mu, j)$ where $i, j \in B D, \mu \in \mathcal{M}$ such that $\alpha(\mu)<i<\beta(\mu)$ and $\alpha(\Delta(\mu))<j<\beta(\Delta(\mu))$. We will assume for simplicity, that if $(i, \mu, j) \in B C$ then $(j, \Delta(\mu), i) \in B C$. This allows one to identify connections $(i, \mu, j)$ and $(j, \Delta(\mu), i)$.

For a combinatorial generalized equation $\Omega$, one can canonically associate a system of equations in variables $h_{1}, \ldots, h_{\rho}$ over $F(A)$ (variables $h_{i}$ are sometimes called items). This system is called a generalized equation, and (slightly abusing the language) we denote it by the same symbol $\Omega$. The generalized equation $\Omega$ consists of the following three types of equations.

1. Each pair of dual variable bases $(\lambda, \Delta(\lambda))$ provides an equation

$$
\left[h_{\alpha(\lambda)} h_{\alpha(\lambda)+1} \ldots h_{\beta(\lambda)-1}\right]^{\varepsilon(\lambda)}=\left[h_{\alpha(\Delta(\lambda))} h_{\alpha(\Delta(\lambda))+1} \ldots h_{\beta(\Delta(\lambda))-1}\right]^{\varepsilon(\Delta(\lambda))} .
$$

These equations are called basic equations.

2. For each constant base $b$ we write down a coefficient equation

$$
h_{\alpha(b)}=a,
$$

where $a \in A^{ \pm 1}$ is the constant associated with $b$.

3. Every boundary connection $(p, \lambda, q)$ gives rise to a boundary equation

$$
\left[h_{\alpha(\lambda)} h_{\alpha(\lambda)+1} \ldots h_{p-1}\right]=\left[h_{\alpha(\Delta(\lambda))} h_{\alpha(\Delta(\lambda))+1} \ldots h_{q-1}\right],
$$

if $\varepsilon(\lambda)=\varepsilon(\Delta(\lambda))$ and

$$
\left[h_{\alpha(\lambda)} h_{\alpha(\lambda)+1} \ldots h_{p-1}\right]=\left[h_{q} h_{q+1} \ldots h_{\beta(\Delta(\lambda))-1}\right]^{-1}
$$


if $\varepsilon(\lambda)=-\varepsilon(\Delta(\lambda))$.

REMARK 2. We assume that every generalized equation comes associated with a combinatorial one;

Example. Consider as an example the Malcev equation $[x, y][b, a]=1$, where $a, b \in A$. Consider the following solution of this equation:

$$
x^{\phi}=\left(\left(b^{n_{1}} a\right)^{n_{2}} b\right)^{n_{3}} b^{n_{1}} a, \quad y^{\phi}=\left(b^{n_{1}} a\right)^{n_{2}} b .
$$

Fig. 3 shows the cancellation tree and the generalized equation for this solution. This generalized equation has ten variables $h_{1}, \ldots, h_{10}$ and eleven boundaries. The
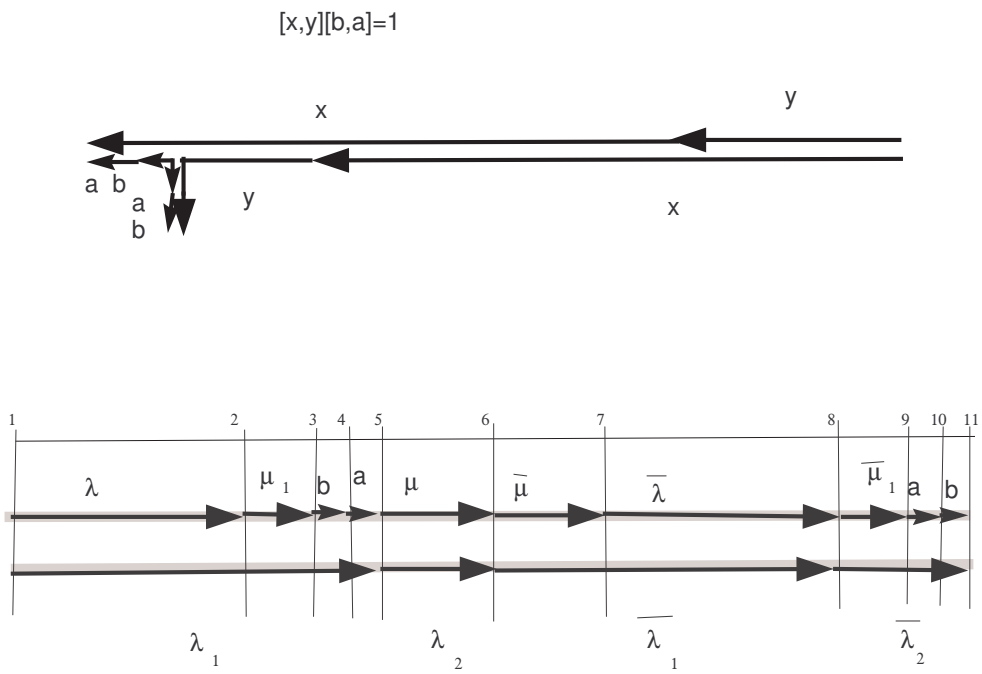

FiguRE 3. A cancellation tree and the generalized equation corresponding to this tree for the equation $[x, y][b, a]=1$.

system of basic equations for this generalized equation is the following

$$
h_{1}=h_{7}, h_{2}=h_{8}, h_{5}=h_{6}, h_{1} h_{2} h_{3} h_{4}=h_{6} h_{7}, h_{5}=h_{8} h_{9} h_{10} .
$$

The system of coefficient equations is

$$
h_{3}=b, h_{4}=a, h_{9}=a, h_{10}=b .
$$

Definition 4.2. Let $\Omega(h)=\left\{L_{1}(h)=R_{1}(h), \ldots, L_{s}(h)=R_{s}(h)\right\}$ be a generalized equation in variables $h=\left(h_{1}, \ldots, h_{\rho}\right)$ with constants from $A^{ \pm 1}$. A sequence of reduced nonempty words $U=\left(U_{1}(A), \ldots, U_{\rho}(A)\right)$ in the alphabet $A^{ \pm 1}$ is a solution of $\Omega$ if:

1) all words $L_{i}(U), R_{i}(U)$ are reduced as written;

2) $L_{i}(U)=R_{i}(U), \quad i=1, \ldots s$.

The notation $(\Omega, U)$ means that $U$ is a solution of the generalized equation $\Omega$. 
REMARK 3. Notice that a solution $U$ of a generalized equation $\Omega$ can be viewed as a solution of $\Omega$ in the free monoid $F_{m o n}\left(A^{ \pm 1}\right)$ (i.e., the equalities $L_{i}(U)=R_{i}(U)$ are graphical) which satisfies an additional condition $U \in F(A) \leq F_{m o n}\left(A^{ \pm 1}\right)$.

Obviously, each solution $U$ of $\Omega$ gives rise to a solution of $\Omega$ in the free group $F(A)$. The converse does not hold in general, i.e., it might happen that $U$ is a solution of $\Omega$ in $F(A)$ but not in $F_{m o n}\left(A^{ \pm 1}\right)$, i.e., all equalities $L_{i}(U)=R_{i}(U)$ hold only after a free reduction but not graphically. We introduce the following notation which will allow us to distinguish in which structure $\left(F_{m o n}\left(A^{ \pm 1}\right)\right.$ or $\left.F(A)\right)$ we are looking for solutions for $\Omega$.

If

$$
S=\left\{L_{1}(h)=R_{1}(h), \ldots, L_{s}(h)=R_{s}(h)\right\}
$$

is an arbitrary system of equations with constants from $A^{ \pm 1}$, then by $S^{*}$ we denote the system of equations

$$
S^{*}=\left\{L_{1}(h) R_{1}(h)^{-1}=1, \ldots, L_{s}(h) R_{s}(h)^{-1}=1\right\}
$$

over the free group $F(A)$.

Definition 4.3. A generalized equations $\Omega$ is called formally consistent if it satisfies the following conditions.

1) If $\varepsilon(\mu)=-\varepsilon(\Delta(\mu))$, then the bases $\mu$ and $\Delta(\mu)$ do not intersect, i.e. non of the the items $h_{\alpha(\mu)}, h_{\beta(\mu)-1}$ is contained in $\Delta(\mu)$.

2) If two boundary equations have respective parameters $(p, \lambda, q)$ and $\left(p_{1}, \lambda, q_{1}\right)$ with $p \leq p_{1}$, then $q \leq q_{1}$ in the case when $\varepsilon(\lambda) \varepsilon(\Delta(\lambda))=1$, and $q \geq q_{1}$ in the case $\varepsilon(\lambda) \varepsilon(\Delta(\lambda))=-1$, in particular, if $p=p_{1}$ then $q=q_{1}$.

3) Let $\mu$ be a base such that $\alpha(\mu)=\alpha(\Delta(\mu)$ ) (in this case we say that bases $\mu$ and $\Delta(\mu)$ form a matched pair of dual bases). If $(p, \mu, q)$ is a boundary connection related to $\mu$ then $p=q$.

4) A variable cannot occur in two distinct coefficient equations, i.e., any two constant bases with the same left end-point are labelled by the same letter from $A^{ \pm 1}$.

5) If $h_{i}$ is a variable from some coefficient equation, and if $\left(i, \mu, q_{1}\right),(i+$ $\left.1, \mu, q_{2}\right)$ are boundary connections, then $\left|q_{1}-q_{2}\right|=1$.

LEMma 4.4. (1) If a generalized equation $\Omega$ has a solution then $\Omega$ is formally consistent;

(2) There is an algorithm which for every generalized equation checks whether it is formally consistent or not.

The proof is easy and we omit it.

REMARK 4. In the sequel we consider only formally consistent generalized equations.

It is convenient to visualize a generalized equation $\Omega$ as follows. 


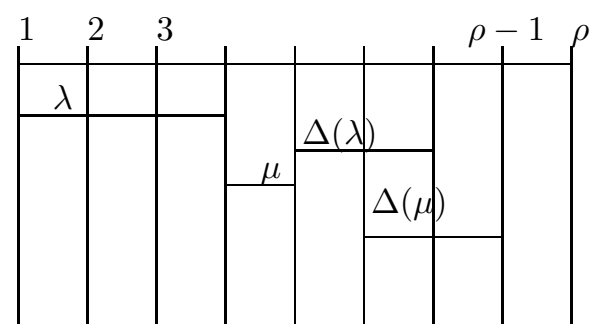

4.2. Reduction to generalized equations. In this section, following Makanin 51, we show how, for a given finite system of equations $S(X, A)=1$ over a free group $F(A)$, one can canonically associate a finite collection of generalized equations $\mathcal{G} E(S)$ with constants from $A^{ \pm 1}$, which to some extent describes all solutions of the system $S(X, A)=1$.

Let $S(X, A)=1$ be a finite system of equations $S_{1}=1, \ldots, S_{m}=1$ over a free group $F(A)$. We write $S(X, A)=1$ in the form

$$
\begin{gathered}
r_{11} r_{12} \ldots r_{1 l_{1}}=1 \\
r_{21} r_{22} \ldots r_{2 l_{2}}=1, \\
\ldots \\
r_{m 1} r_{m 2} \ldots r_{m l_{m}}=1
\end{gathered}
$$

where $r_{i j}$ are letters in the alphabet $X^{ \pm 1} \cup A^{ \pm 1}$.

A partition table $T$ of the system above is a set of reduced words

$$
T=\left\{V_{i j}\left(z_{1}, \ldots, z_{p}\right)\right\} \quad\left(1 \leq i \leq m, 1 \leq j \leq l_{i}\right)
$$

from a free group $F[Z]=F(A \cup Z)$, where $Z=\left\{z_{1}, \ldots, z_{p}\right\}$, which satisfies the following conditions:

1) The equality $V_{i 1} V_{i 2} \cdots V_{i l_{i}}=1,1 \leq i \leq m$, holds in $F[Z]$;

2) $\left|V_{i j}\right| \leq l_{i}-1$

3) if $r_{i j}=a \in A^{ \pm 1}$, then $V_{i j}=a$.

Since $\left|V_{i j}\right| \leq l_{i}-1$ then at most $|S|=\sum_{i=1}^{m}\left(l_{i}-1\right) l_{i}$ different letters $z_{i}$ can occur in a partition table of the equation $S(X, A)=1$. Therefore we will always assume that $p \leq d(S)$.

Each partition table encodes a particular type of cancellation that happens when one substitutes a particular solution $W(A) \in F(A)$ into $S(X, A)=1$ and then freely reduces the words in $S(W(A), A)$ into the empty word.

Lemma 4.5. Let $S(X, A)=1$ be a finite system of equations over $F(A)$. Then

(1) the set $P T(S)$ of all partition tables of $S(X, A)=1$ is finite, and its cardinality is bounded by a number which depends only on $|S(X, A)|$;

(2) one can effectively enumerate the set $P T(S)$.

Proof. Since the words $V_{i j}$ have bounded length, one can effectively enumerate the finite set of all collections of words $\left\{V_{i j}\right\}$ in $F[Z]$ which satisfy the conditions $2), 3)$ above. Now for each such collection $\left\{V_{i j}\right\}$, one can effectively check whether the equalities $V_{i 1} V_{i 2} \ldots V_{i l_{i}}=1,1 \leq i \leq m$ hold in the free group $F[Z]$ or not. This allows one to list effectively all partition tables for $S(X, A)=1$. 
To each partition table $T=\left\{V_{i j}\right\}$ one can assign a generalized equation $\Omega_{T}$ in the following way (below we use the notation $\doteq$ for graphical equality). Consider the following word $V$ in $M\left(A^{ \pm 1} \cup Z^{ \pm 1}\right)$ :

$$
V \doteq V_{11} V_{12} \cdots V_{1 l_{1}} \cdots V_{m 1} V_{m 2} \cdots V_{m l_{m}}=y_{1} \cdots y_{\rho}
$$

where $y_{i} \in A^{ \pm 1} \cup Z^{ \pm 1}$ and $\rho=d(V)$ is the length of $V$. Then the generalized equation $\Omega_{T}=\Omega_{T}(h)$ has $\rho+1$ boundaries and $\rho$ variables $h_{1}, \ldots, h_{\rho}$ which are denoted by $h=\left(h_{1}, \ldots, h_{\rho}\right)$.

Now we define bases of $\Omega_{T}$ and the functions $\alpha, \beta, \varepsilon$.

Let $z \in Z$. For any two distinct occurrences of $z$ in $V$ as

$$
y_{i}=z^{\varepsilon_{i}}, \quad y_{j}=z^{\varepsilon_{j}} \quad\left(\varepsilon_{i}, \varepsilon_{j} \in\{1,-1\}\right)
$$

we introduce a pair of dual variable bases $\mu_{z, i}, \mu_{z, j}$ such that $\Delta\left(\mu_{z, i}\right)=\mu_{z, j}$ (say, if $i<j$ ). Put

$$
\alpha\left(\mu_{z, i}\right)=i, \quad \beta\left(\mu_{z, i}\right)=i+1, \quad \epsilon\left(\mu_{z, i}\right)=\varepsilon_{i} .
$$

The basic equation that corresponds to this pair of dual bases is $h_{i}^{\varepsilon_{i}}=h_{j}^{\varepsilon_{j}}$.

Let $x \in X$. For any two distinct occurrences of $x$ in $S(X, A)=1$ as

$$
r_{i, j}=x^{\varepsilon_{i j}}, \quad r_{s, t}=x^{\varepsilon_{s t}} \quad\left(\varepsilon_{i j}, \varepsilon_{s t} \in\{1,-1\}\right)
$$

we introduce a pair of dual bases $\mu_{x, i, j}$ and $\mu_{x, s, t}$ such that $\Delta\left(\mu_{x, i, j}\right)=\mu_{x, s, t}$ (say, if $(i, j)<(s, t)$ in the left lexicographic order). Now let $V_{i j}$ occurs in the word $V$ as a subword

Then we put

$$
V_{i j}=y_{c} \cdots y_{d}
$$

$$
\alpha\left(\mu_{x, i, j}\right)=c, \quad \beta\left(\mu_{x, i, j}\right)=d+1, \quad \epsilon\left(\mu_{x, i, j}\right)=\varepsilon_{i j} .
$$

The basic equation which corresponds to these dual bases can be written in the form

$$
\left[h_{\alpha\left(\mu_{x, i, j}\right)} \ldots h_{\beta\left(\mu_{x, i, j}\right)-1}\right]^{\varepsilon_{i j}}=\left[h_{\alpha\left(\mu_{x, s, t}\right)} \ldots h_{\beta\left(\mu_{x, s, t}\right)-1}\right]^{\varepsilon_{s t}} .
$$

Let $r_{i j}=a \in A^{ \pm 1}$. In this case we introduce a constant base $\mu_{i j}$ with the label $a$. If $V_{i j}$ occurs in $V$ as $V_{i j}=y_{c}$, then we put

$$
\alpha\left(\mu_{i j}\right)=c, \beta\left(\mu_{i j}\right)=c+1 .
$$

The corresponding coefficient equation is written as $h_{c}=a$.

The list of boundary connections here (and hence the boundary equations) is empty. This defines the generalized equation $\Omega_{T}$. Put

$$
\mathcal{G} E(S)=\left\{\Omega_{T} \mid T \text { is a partition table for } S(X, A)=1\right\} .
$$

Then $\mathcal{G} E(S)$ is a finite collection of generalized equations which can be effectively constructed for a given $S(X, A)=1$.

For a generalized equation $\Omega$ we can also consider the same system of equations in a free group. We denote this system by $\Omega^{*}$. By $F_{R(\Omega)}$ we denote the coordinate group of $\Omega^{*}$. Now we explain relations between the coordinate groups of $S(X, A)=1$ and $\Omega_{T}^{*}$.

For a letter $x$ in $X$ we choose an arbitrary occurrence of $x$ in $S(X, A)=1$ as

$$
r_{i j}=x^{\varepsilon_{i j}} .
$$

Let $\mu=\mu_{x, i, j}$ be the base that corresponds to this occurrence of $x$. Then $V_{i j}$ occurs in $V$ as the subword

$$
V_{i j}=y_{\alpha(\mu)} \ldots y_{\beta(\mu)-1} \text {. }
$$


Define a word $P_{x}(h) \in F[h]$ (where $\left.h=\left\{h_{1}, \ldots, h_{\rho}\right\}\right)$ as

$$
P_{x}(h, A)=h_{\alpha(\mu)} \ldots h_{\beta(\mu)-1}^{\varepsilon_{i j}},
$$

and put

$$
P(h)=\left(P_{x_{1}}, \ldots, P_{x_{n}}\right) .
$$

The tuple of words $P(h)$ depends on a choice of occurrences of letters from $X$ in $V$. It follows from the construction above that the map $X \rightarrow F[h]$ defined by $x \rightarrow P_{x}(h, A)$ gives rise to an $F$-homomorphism

$$
\pi: F_{R(S)} \rightarrow F_{R\left(\Omega_{T}\right)} .
$$

Observe that the image $\pi(x)$ in $F_{R\left(\Omega_{T}\right)}$ does not depend on a particular choice of the occurrence of $x$ in $S(X, A)$ (the basic equations of $\Omega_{T}$ make these images equal). Hence $\pi$ depends only on $\Omega_{T}$.

Now we relate solutions of $S(X, A)=1$ with solutions of generalized equations from $\mathcal{G} E(S)$. Let $W(A)$ be a solution of $S(X, A)=1$ in $F(A)$. If in the system (14) we make the substitution $\sigma: X \rightarrow W(A)$, then

$$
\left(r_{i 1} r_{i 2} \ldots r_{i l_{i}}\right)^{\sigma}=r_{i 1}^{\sigma} r_{i 2}^{\sigma} \ldots r_{i l_{i}}^{\sigma}=1
$$

in $F(A)$ for every $i=1, \ldots, m$. Hence every product $R_{i}=r_{i 1}^{\sigma} r_{i 2}^{\sigma} \ldots r_{i l_{i}}^{\sigma}$ can be reduced to the empty word by a sequence of free reductions. Let us fix a particular reduction process for each $R_{i}$. Denote by $\tilde{z}_{1}, \ldots, \tilde{z}_{p}$ all the (maximal) non-trivial subwords of $r_{i j}^{\sigma}$ that cancel out in some $R_{i}(i=1, \ldots, m)$ during the chosen reduction process. Since every word $r_{i j}^{\sigma}$ in this process cancels out completely, that implies that

$$
r_{i j}^{\sigma}=V_{i j}\left(\tilde{z}_{1}, \ldots, \tilde{z}_{p}\right)
$$

for some reduced words $V_{i j}(Z)$ in variables $Z=\left\{z_{1}, \ldots, z_{p}\right\}$. Moreover, the equality above is graphical. Observe also that if $r_{i j}=a \in A^{ \pm 1}$ then $r_{i j}^{\sigma}=a$ and we have $V_{i j}=a$. Since every word $r_{i j}^{\sigma}$ in $R_{i}$ has at most one cancellation with any other word $r_{i k}^{\sigma}$ and does not have cancellation with itself, we have $\left|V_{i j}\right| \leq l_{i}-1$. This shows that the set $T=\left\{V_{i j}\right\}$ is a partition table for $S(X, A)=1$. Obviously,

$$
U(A)=\left(\tilde{z}_{1}, \ldots, \tilde{z}_{p}\right)
$$

is the solution of the generalized equation $\Omega_{T}$, which is induced by $W(A)$. From the construction of the map $P(H)$ we deduce that $W(A)=P(U(A))$.

The reverse is also true: if $U(A)$ is an arbitrary solution of the generalized equation $\Omega_{T}$, then $P(U(A))$ is a solution of $S(X, A)=1$.

We summarize the discussion above in the following lemma, which is essentially due to Makanin [51].

Lemma 4.6. For a given system of equations $S(X, A)=1$ over a free group $F=F(A)$, one can effectively construct a finite set

$$
\mathcal{G} E(S)=\left\{\Omega_{T} \mid T \text { is a partition table for } S(X, A)=1\right\}
$$

of generalized equations such that

(1) If the set $\mathcal{G} E(S)$ is empty, then $S(X, A)=1$ has no solutions in $F(A)$;

(2) for each $\Omega(H) \in \mathcal{G} E(S)$ and for each $x \in X$ one can effectively find a word $P_{x}(H, A) \in F[H]$ of length at most $|H|$ such that the map $x: \rightarrow P_{x}(H, A)$ $(x \in X)$ gives rise to an F-homomorphism $\pi_{\Omega}: F_{R(S)} \rightarrow F_{R(\Omega)}$; 
(3) for any solution $W(A) \in F(A)^{n}$ of the system $S(X, A)=1$ there exists $\Omega(H) \in \mathcal{G} E(S)$ and a solution $U(A)$ of $\Omega(H)$ such that $W(A)=P(U(A))$, where $P(H)=\left(P_{x_{1}}, \ldots, P_{x_{n}}\right)$, and this equality is graphical;

(4) for any $F$-group $\tilde{F}$, if a generalized equation $\Omega(H) \in \mathcal{G} E(S)$ has a solution $\tilde{U}$ in $\tilde{F}$, then $P(\tilde{U})$ is a solution of $S(X, A)=1$ in $\tilde{F}$.

COROLlary 4.7. In the notations of Lemma 4.6 for any solution $W(A) \in$ $F(A)^{n}$ of the system $S(X, A)=1$ there exists $\Omega(H) \in \mathcal{G} E(S)$ and a solution $U(A)$ of $\Omega(H)$ such that the following diagram commutes.

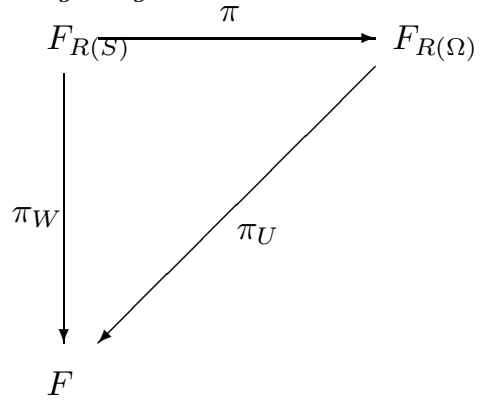

4.3. Generalized equations with parameters. In this section, following 62 and 36, we consider generalized equations with parameters. These kinds of equations appear naturally in Makanin's type rewriting processes and provide a convenient tool to organize induction properly.

Let $\Omega$ be a generalized equation. An item $h_{i}$ belongs to a base $\mu$ (and, in this event, $\mu$ contains $h_{i}$ ) if $\alpha(\mu) \leq i \leq \beta(\mu)-1$. An item $h_{i}$ is constant if it belongs to a constant base, $h_{i}$ is free if it does not belong to any base. By $\gamma\left(h_{i}\right)=\gamma_{i}$ we denote the number of bases which contain $h_{i}$. We call $\gamma_{i}$ the degree of $h_{i}$.

A boundary $i$ crosses (or intersects) the base $\mu$ if $\alpha(\mu)<i<\beta(\mu)$. A boundary $i$ touches the base $\mu$ (or $i$ is an end-point of $\mu$ ) if $i=\alpha(\mu)$ or $i=\beta(\mu)$. A boundary is said to be open if it crosses at least one base, otherwise it is called closed. We say that a boundary $i$ is tied (or bound) by a base $\mu$ (or $\mu$-tied) if there exists a boundary connection $(p, \mu, q)$ such that $i=p$ or $i=q$. A boundary is free if it does not touch any base and it is not tied by a boundary connection.

A set of consecutive items $[i, j]=\left\{h_{i}, \ldots, h_{i+j-1}\right\}$ is called a section. A section is said to be closed if the boundaries $i$ and $i+j$ are closed and all the boundaries between them are open. A base $\mu$ is contained in a base $\lambda$ if $\alpha(\lambda) \leq \alpha(\mu)<\beta(\mu) \leq$ $\beta(\lambda)$. If $\mu$ is a base then by $\sigma(\mu)$ we denote the section $[\alpha(\mu), \beta(\mu)]$ and by $h(\mu)$ we denote the product of items $h_{\alpha(\mu)} \ldots h_{\beta(\mu)-1}$. In general for a section $[i, j]$ by $h[i, j]$ we denote the product $h_{i} \ldots h_{j-1}$.

Definition 4.8. Let $\Omega$ be a generalized equation. If the set $\Sigma=\Sigma_{\Omega}$ of all closed sections of $\Omega$ is partitioned into a disjoint union of subsets

$$
\Sigma_{\Omega}=V \Sigma \cup P \Sigma \cup C \Sigma,
$$

then $\Omega$ is called a generalized equation with parameters or a parametric generalized equation. Sections from $V \Sigma, P \Sigma$, and $C \Sigma$ are called correspondingly, variable, parametric, and constant sections. To organize the branching process properly, we usually divide variable sections into two disjoint parts:

$$
V \Sigma=A \Sigma \cup N A \Sigma
$$


Sections from $A \Sigma$ are called active, and sections from $N A \Sigma$ are non-active. In the case when partition (16) is not specified we assume that $A \Sigma=V \Sigma$. Thus, in general, we have a partition

$$
\Sigma_{\Omega}=A \Sigma \cup N A \Sigma \cup P \Sigma \cup C \Sigma
$$

If $\sigma \in \Sigma$, then every base or item from $\sigma$ is called active, non-active, parametric, or constant, with respect to the type of $\sigma$.

We will see later that every parametric generalized equation can be written in a particular standard form.

DeFINITION 4.9. We say that a parametric generalized equation $\Omega$ is in a standard form if the following conditions hold:

1) all non-active sections from $N A \Sigma_{\Omega}$ are located to the right of all active sections from $A \Sigma$, all parametric sections from $P \Sigma_{\Omega}$ are located to the right of all non-active sections, and all constant sections from $C \Sigma$ are located to the right of all parametric sections; namely, there are numbers $1 \leq \rho_{A} \leq \rho_{N A} \leq \rho_{P} \leq \rho_{C} \leq \rho_{=} \rho_{\Omega}$ such that $\left[1, \rho_{A}+1\right],\left[\rho_{A}+1, \rho_{N A}+1\right]$, $\left[\rho_{N A}+1, \rho_{P}+1\right]$, and $\left[\rho_{P}+1, \rho_{\Omega}+1\right]$ are, correspondingly, unions of all active, all non-active, all parametric, and all constant sections;

2) for every letter $a \in A^{ \pm 1}$ there is at most one constant base in $\Omega$ labelled by $a$, and all such bases are located in the $C \Sigma$;

3 ) every free variable (item) $h_{i}$ of $\Omega$ is located in $C \Sigma$.

Sometimes, we label the item associated with some letter by this letter.

Now we describe a typical method for constructing generalized equations with parameters starting with a system of ordinary group equations with constants from $A$.

4.4. Parametric generalized equations corresponding to group equations. Let

$$
S\left(X, Y_{1}, Y_{2}, \ldots, Y_{k}, A\right)=1
$$

be a finite system of equations with constants from $A^{ \pm 1}$ and with the set of variables partitioned into a disjoint union

$$
X \cup Y_{1} \cup \ldots \cup Y_{k}
$$

Denote by $\mathcal{G} E(S)$ the set of generalized equations corresponding to $S=1$ from Lemma 4.6] Put $Y=Y_{1} \cup \ldots \cup Y_{k}$. Let $\Omega \in \mathcal{G} E(S)$. Recall that every base $\mu$ occurs in $\Omega$ either related to some occurrence of a variable from $X \cup Y$ in the system $S(X, Y, A)=1$, or related to an occurrence of a letter $z \in Z$ in the word $V$ (see Lemma 4.5), or is a constant base. If $\mu$ corresponds to a variable $x \in X\left(y \in Y_{i}\right)$ then we say that $\mu$ is an $X$-base $\left(Y_{i}\right.$-base). Sometimes we refer to $Y_{i}$-bases as to $Y$ bases. For a base $\mu$ of $\Omega$ denote by $\sigma_{\mu}$ the section $\sigma_{\mu}=[\alpha(\mu), \beta(\mu)]$. Observe that the section $\sigma_{\mu}$ is closed in $\Omega$ for every $X$-base, or $Y$-base. If $\mu$ is an $X$-base $(Y$-base or $Y_{i}$-base), then the section $\sigma_{\mu}$ is called an $X$-section ( $Y$-section or $Y_{i}$-section). If $\mu$ is a constant base and the section $\sigma_{\mu}$ is closed then we call $\sigma_{\mu}$ a constant section. Using the derived transformation $\mathrm{D} 2$ we transport all closed $Y_{1}$-sections to the right end of the generalized equations behind all the sections of the equation (in an arbitrary order), then we transport all $Y_{2}$-sections and put them behind all $Y_{1}$-sections, and so on. Eventually, we transport all $Y$-sections to the very end of 
the interval and they appear there with respect to the partition (19). After that we take all the constant sections and put them behind all the parametric sections. Now, let $A \Sigma$ be the set of all $X$-sections, $N A \Sigma=\emptyset, P \Sigma$ be the set of all $Y$-sections, and $C \Sigma$ be the set of all constant sections. This defines a parametric generalized equation $\Omega=\Omega_{Y}$ with parameters corresponding to the set of variables $Y$. If the partition of variables (19) is fixed we will omit $Y$ in the notation above and call $\Omega$ the parameterized equation obtained from $\Omega$. Denote by

$$
\mathcal{G} E_{\text {par }}(\Omega)=\left\{\Omega_{Y} \mid \Omega \in \mathcal{G} E(\Omega)\right\}
$$

the set of all parameterized equations of the system (18).

\section{Elimination process: construction of $T(\Omega)$}

In 36 and 40 , Section 5, we described a general process of transformations of a generalized equation and construction of fundamental sequences of solutions of equations. In those papers we called this process "Makanin-Razborov process", and sometimes it caused a confusion because the process that we described is quite different. Since we use different variations of the process, we prefer to call any such variation here an Elimination process.

5.1. Elementary transformations. In this section we describe elementary transformations of generalized equations which were introduced by Makanin in 51. Recall that we consider only formally consistent equations. In general, an elementary transformation $E T$ associates to a generalized equation $\Omega$ a finite set of generalized equations $E T(\Omega)=\left\{\Omega_{1}, \ldots, \Omega_{r}\right\}$ and a collection of surjective homomorphisms $\theta_{i}: G_{R(\Omega)} \rightarrow G_{R\left(\Omega_{i}\right)}$ such that for every pair $(\Omega, U)$ there exists a unique pair of the type $\left(\Omega_{i}, U_{i}\right)$ for which the following diagram commutes.

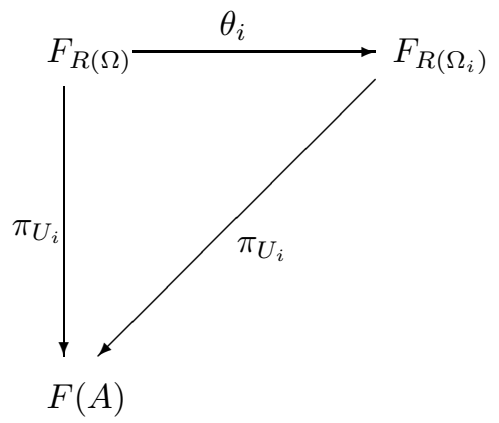

Here $\pi_{U}(X)=U$. Since the pair $\left(\Omega_{i}, U_{i}\right)$ is defined uniquely, we have a welldefined map

$E T:(\Omega, U) \rightarrow\left(\Omega_{i}, U_{i}\right)$.

ET1 (Cutting a base). Suppose $\Omega$ contains a boundary connection $\langle p, \lambda, q\rangle$. Then we replace (cut in $p$ ) the base $\lambda$ by two new bases $\lambda_{1}$ and $\lambda_{2}$ and also replace (cut in $q) \Delta(\lambda)$ by two new bases $\Delta\left(\lambda_{1}\right)$ and $\Delta\left(\lambda_{2}\right)$ such that the following conditions hold.

$$
\begin{aligned}
& \text { If } \varepsilon(\lambda)=\varepsilon(\Delta(\lambda)) \text {, then } \\
& \alpha\left(\lambda_{1}\right)=\alpha(\lambda), \quad \beta\left(\lambda_{1}\right)=p, \quad \alpha\left(\lambda_{2}\right)=p, \quad \beta\left(\lambda_{2}\right)=\beta(\lambda) ; \\
& \alpha\left(\Delta\left(\lambda_{1}\right)\right)=\alpha(\Delta(\lambda)), \quad \beta\left(\Delta\left(\lambda_{1}\right)\right)=q, \quad \alpha\left(\Delta\left(\lambda_{2}\right)\right)=q, \quad \beta\left(\Delta\left(\lambda_{2}\right)\right)=\beta(\Delta(\lambda)) ;
\end{aligned}
$$




$$
\begin{array}{ll}
\text { If } \varepsilon(\lambda)=-\varepsilon(\Delta(\lambda)), \text { then } \\
\begin{aligned}
\alpha\left(\lambda_{1}\right)=\alpha(\lambda), \quad \beta\left(\lambda_{1}\right)=p, & \alpha\left(\lambda_{2}\right)=p, \quad \beta\left(\lambda_{2}\right)=\beta(\lambda) ;
\end{aligned} \\
\alpha\left(\Delta\left(\lambda_{1}\right)\right)=q, \quad \beta\left(\Delta\left(\lambda_{1}\right)\right)=\beta(\Delta(\lambda)), & \alpha\left(\Delta\left(\lambda_{2}\right)\right)=\alpha(\Delta(\lambda)), \quad \beta\left(\Delta\left(\lambda_{2}\right)\right)=q ;
\end{array}
$$

Put $\varepsilon\left(\lambda_{i}\right)=\varepsilon(\lambda), \quad \varepsilon\left(\Delta\left(\lambda_{i}\right)\right)=\varepsilon(\Delta(\lambda)), i=1,2$.

Let $\left(p^{\prime}, \lambda, q^{\prime}\right)$ be a boundary connection in $\Omega$.

If $p^{\prime}<p$, then replace $\left(p^{\prime}, \lambda, q^{\prime}\right)$ by $\left(p^{\prime}, \lambda_{1}, q^{\prime}\right)$.

If $p^{\prime}>p$, then replace $\left(p^{\prime}, \lambda, q^{\prime}\right)$ by $\left(p^{\prime}, \lambda_{2}, q^{\prime}\right)$.

Notice, since the equation $\Omega$ is formally consistent, then the conditions above define boundary connections in the new generalized equation. The resulting generalized equation $\Omega^{\prime}$ is formally consistent. Put $E T(\Omega)=\left\{\Omega^{\prime}\right\}$. Fig. 4 below explains the name of the transformation ET1.

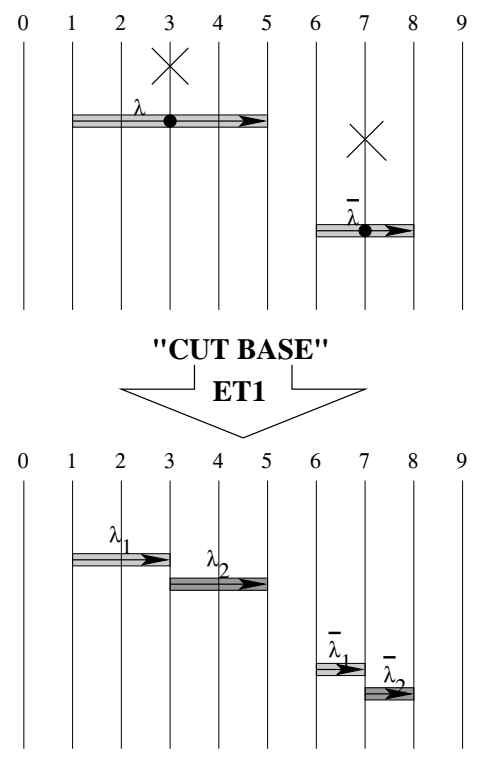

FiguRE 4. Elementary transformation ET1.

ET2 (Transfer of a base). Let a base $\mu$ of a generalized equation $\Omega$ be contained in the base $\lambda$, i.e., $\alpha(\lambda) \leq \alpha(\mu)<\beta(\mu) \leq \beta(\lambda)$ ). Suppose that the boundaries $\alpha(\mu)$ and $\beta(\mu))$ are $\lambda$-tied, i.e., there are boundary connections of the type $\left\langle\alpha(\mu), \lambda, \gamma_{1}\right\rangle$ and $\left\langle\beta(\mu), \lambda, \gamma_{2}\right\rangle$. Suppose also that every $\mu$-tied boundary is $\lambda$-tied. Then we transfer $\mu$ from its location on the base $\lambda$ to the corresponding location on the base $\Delta(\lambda)$ and adjust all the basic and boundary equations (see Fig. 5). More formally, we replace $\mu$ by a new base $\mu^{\prime}$ such that $\alpha\left(\mu^{\prime}\right)=\gamma_{1}, \beta\left(\mu^{\prime}\right)=\gamma_{2}$ and replace each $\mu$-boundary connection $(p, \mu, q)$ with a new one $\left(p^{\prime}, \mu^{\prime}, q\right)$ where $p$ and $p^{\prime}$ come from the $\lambda$-boundary connection $\left(p, \lambda, p^{\prime}\right)$. The resulting equation is denoted by $\Omega^{\prime}=\operatorname{ET} 2(\Omega)$.

ET3 (Removal of a pair of matched bases (see Fig. 6)). Let $\lambda$ and $\Delta(\lambda)$ be a pair of matched bases in $\Omega$. Since $\Omega$ is formally consistent one has $\varepsilon(\lambda)=\varepsilon(\Delta(\lambda)$ ), $\beta(\lambda)=\beta(\Delta(\lambda))$ and every $\lambda$-boundary connection is of the type $(p, \lambda, p)$. Remove the pair of bases $\lambda, \Delta(\lambda)$ with all boundary connections related to $\lambda$. Denote the new generalized equation by $\Omega^{\prime}$. 


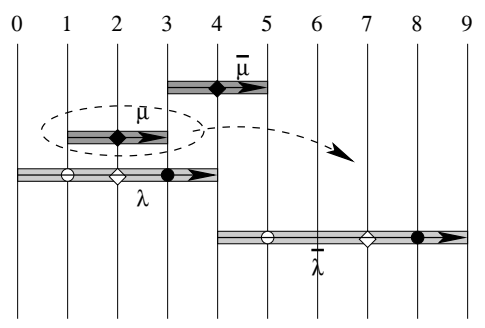

"TRANSFER BASE"
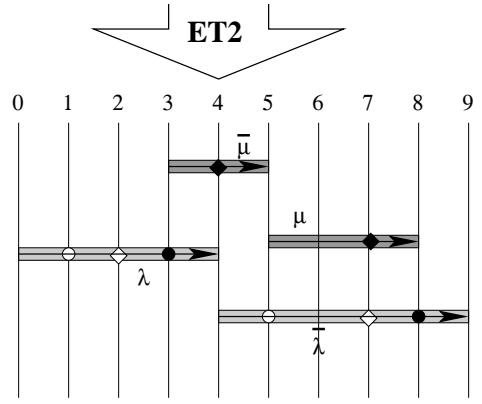

FiguRE 5. Elementary transformation ET2.

Remark. Observe, that for $i=1,2,3 \operatorname{ET} i(\Omega)$ consists of a single equation $\Omega^{\prime}$, such that $\Omega$ and $\Omega^{\prime}$ have the same set of variables $H$, and the identity map $F[H] \rightarrow$ $F[H]$ induces an $F$-isomorphism $F_{R(\Omega)} \rightarrow F_{R\left(\Omega^{\prime}\right)}$. Moreover, $U$ is a solution of $\Omega$ if and only if $U$ is a solution of $\Omega^{\prime}$.

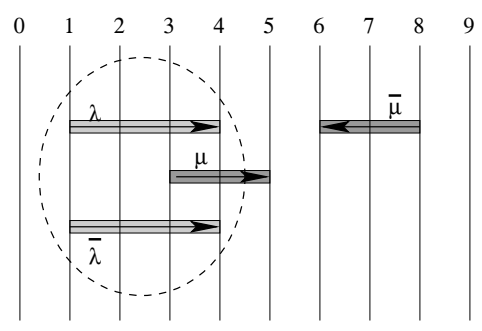

"DELETE MATCHED BASE"

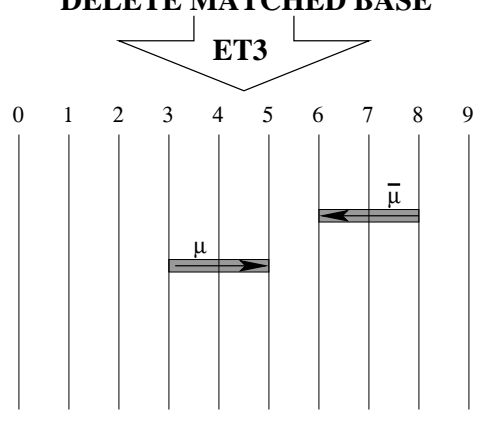

Figure 6. Elementary transformation ET3. 
ET4 (Removal of a lonely base (see Fig. 7)). Suppose in $\Omega$ a variable base $\lambda$ does not intersect any other variable base, i.e., the items $h_{\alpha(\lambda)}, \ldots, h_{\beta(\lambda)-1}$ are contained in only one variable base $\lambda$. Suppose also that all boundaries in $\lambda$ are $\lambda$-tied, i.e., for every $i(\alpha(\lambda)+1 \leq i \leq \beta-1)$ there exists a boundary $b(i)$ such that $(i, \lambda, b(i))$ is a boundary connection in $\Omega$. For convenience we define: $b(\alpha(\lambda))=$ $\alpha(\Delta(\lambda))$ and $b(\beta(\lambda))=\beta(\Delta(\lambda))$ if $\varepsilon(\lambda) \varepsilon(\Delta(\lambda))=1$, and $b(\alpha(\lambda))=\beta(\Delta(\lambda))$ and $b(\beta(\lambda))=\alpha(\Delta(\lambda))$ if $\varepsilon(\lambda) \varepsilon(\Delta(\lambda))=-1$.

The transformation ET4 carries $\Omega$ into a unique generalized equation $\Omega_{1}$ which is obtained from $\Omega$ by deleting the pair of bases $\lambda$ and $\Delta(\lambda)$; deleting all the boundaries $\alpha(\lambda)+1, \ldots, \beta(\lambda)-1$ ( and renaming the rest $\beta(\lambda)-\alpha(\lambda)-1$ boundaries) together with all $\lambda$-boundary connections; replacing every constant base $\theta$ which is contained in $\lambda$ by a constant base $\theta^{\prime}$ with the same label as $\theta$ and such that $\alpha\left(\theta^{\prime}\right)=b(\alpha(\theta)), \beta\left(\theta^{\prime}\right)=b(\beta(\theta))$.

We define the homomorphism $\pi: F_{R(\Omega)} \rightarrow F_{R\left(\Omega^{\prime}\right)}$ as follows: $\pi\left(h_{j}\right)=h_{j}$ if $j<\alpha(\lambda)$ or $j \geq \beta(\lambda)$

$$
\pi\left(h_{i}\right)= \begin{cases}h_{b(i)} \ldots h_{b(i)-1}, & \text { if } \varepsilon(\lambda)=\varepsilon(\Delta \lambda), \\ h_{b(i)} \ldots h_{b(i-1)-1}, & \text { if } \varepsilon(\lambda)=-\varepsilon(\Delta \lambda)\end{cases}
$$

for $\alpha+1 \leq i \leq \beta(\lambda)-1$. It is not hard to see that $\pi$ is an $F$-isomorphism.
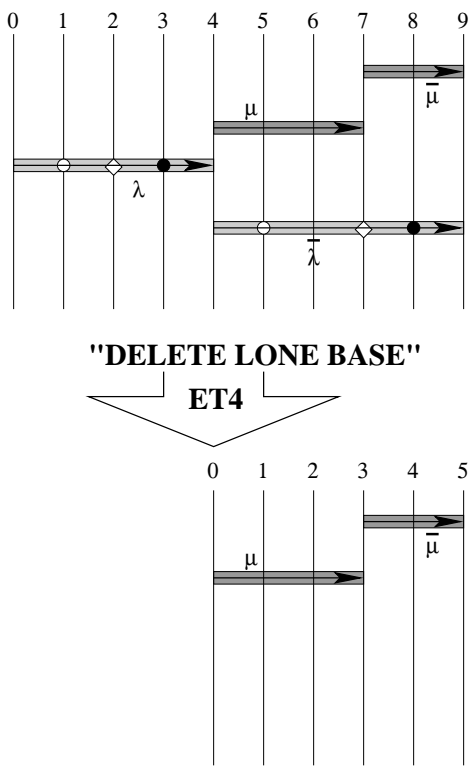

FiguRE 7. Elementary transformation ET4.

ET5 (Introduction of a boundary (see Fig. 8)). Suppose a point $p$ in a base $\lambda$ is not $\lambda$-tied. The transformation ET5 $\lambda$-ties it in all possible ways, producing finitely many different generalized equations. To this end, let $q$ be a boundary on $\Delta(\lambda)$. Then we perform one of the following two transformations:

1. Introduce the boundary connection $\langle p, \lambda, q\rangle$ if the resulting equation $\Omega_{q}$ is formally consistent. In this case the corresponding $F$-homomorphism $\pi_{q}: F_{R(\Omega)}$ into $F_{R\left(\Omega_{q}\right)}$ is induced by the identity isomorphism on $F[H]$. Observe that $\pi_{q}$ is not necessary an isomorphism. 
2. Introduce a new boundary $q^{\prime}$ between $q$ and $q+1$ (and rename all the boundaries); introduce a new boundary connection $\left(p, \lambda, q^{\prime}\right)$. Denote the resulting equation by $\Omega_{q}^{\prime}$. In this case the corresponding $F$-homomorphism $\pi_{q^{\prime}}: F_{R(\Omega)}$ into $F_{R\left(\Omega_{q^{\prime}}\right)}$ is induced by the map $\pi(h)=h$, if $h \neq h_{q}$, and $\pi\left(h_{q}\right)=h_{q^{\prime}} h_{q^{\prime}+1}$. Observe that $\pi_{q^{\prime}}$ is an $F$-isomorphism.
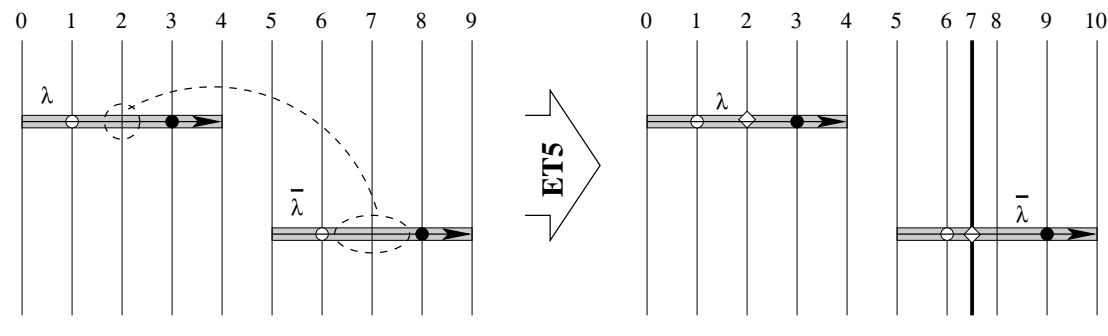

"ADD BOUNDARY CONNECTION"
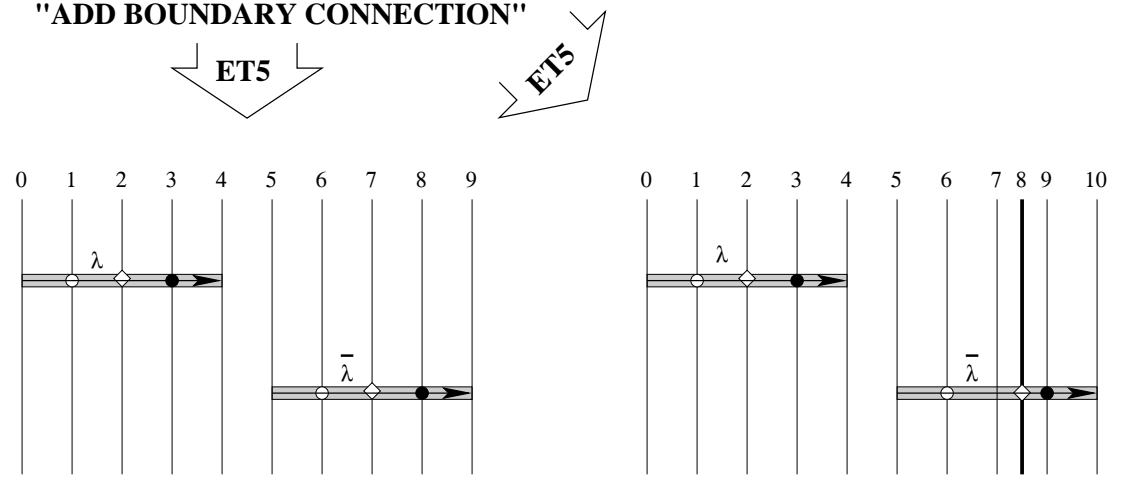

FIGURE 8. Elementary transformation ET5.

Let $\Omega$ be a generalized equation and $E$ be an elementary transformation. By $E(\Omega)$ we denote a generalized equation obtained from $\Omega$ by the elementary transformation $E$ (perhaps several such equations) if $E$ is applicable to $\Omega$, otherwise we put $E(\Omega)=\Omega$. By $\phi_{E}: F_{R(\Omega)} \rightarrow F_{R(E(\Omega))}$ we denote the canonical homomorphism of the coordinate groups (which has been described above in the case $E(\Omega) \neq \Omega$ ), otherwise, the identical isomorphism.

LEMMA 5.1. There exists an algorithm which for every generalized equation $\Omega$ and every elementary transformation $E$ determines whether the canonical homomorphism $\phi_{E}: F_{R(\Omega)} \rightarrow F_{R(E(\Omega))}$ is an isomorphism or not.

Proof. The only non-trivial case is when $E=E 5$ and no new boundaries were introduced. In this case $E(\Omega)$ is obtained from $\Omega$ by adding a new particular equation, say $s=1$, which is effectively determined by $\Omega$ and $E(\Omega)$. In this event, the coordinate group

$$
F_{R(E(\Omega))}=F_{R(\Omega \cup\{s\})}
$$

is a quotient group of $F_{R(\Omega)}$. Now $\phi_{E}$ is an isomorphism if and only if $R(\Omega)=$ $R(\Omega \cup\{s\})$, or, equivalently, $s \in R(\Omega)$. The latter condition holds if and only if $s$ vanishes on all solutions of the system of (group-theoretic) equations $\Omega=1$ in $F$, i.e., if the following formula holds in $F$ :

$$
\forall x_{1} \ldots \forall x_{\rho}\left(\Omega\left(x_{1}, \ldots, x_{\rho}\right)=1 \rightarrow s\left(x_{1}, \ldots, x_{\rho}\right)=1\right) .
$$


This can be checked effectively, since the universal theory of a free group $F$ is decidable ([52 $)$.

5.2. Derived transformations and auxiliary transformations. In this section we describe several useful transformations of generalized equations. Some of them can be realized as finite sequences of elementary transformations, we call them derived transformations. Other transformations result in equivalent generalized equations but cannot be realized by finite sequences of elementary moves.

D1 (Closing a section).

Let $\sigma$ be a section of $\Omega$. The transformation D1 makes the section $\sigma$ closed. To perform D1 we introduce boundary connections (transformations ET5) through the end-points of $\sigma$ until these end-points are tied by every base containing them, and then cut through the end-points all the bases containing them (transformations ET1)(see Fig. 9)
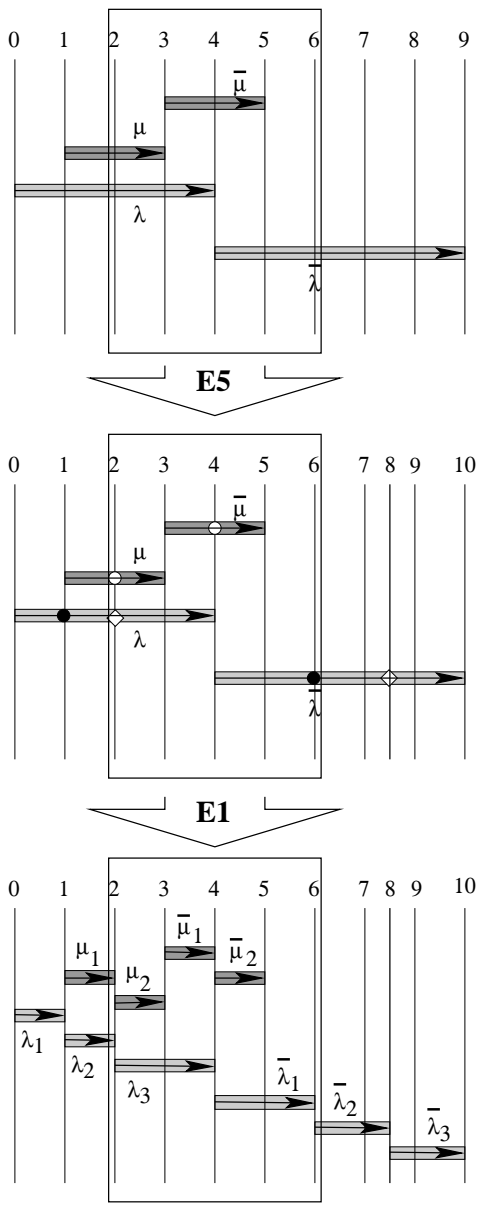

FiguRE 9. Derived transformation D1.

D2 (Transporting a closed section).

Let $\sigma$ be a closed section of a generalized equation $\Omega$. We cut $\sigma$ out of the interval $\left[1, \rho_{\Omega}\right]$ together with all the bases and boundary connections on $\sigma$ and put 
$\sigma$ at the end of the interval or between any two consecutive closed sections of $\Omega$. After that we correspondingly re-enumerate all the items and boundaries of the latter equation to bring it to the proper form. Clearly, the original equation $\Omega$ and the new one $\Omega^{\prime}$ have the same solution sets and their coordinate groups are isomorphic (see Fig. 10)

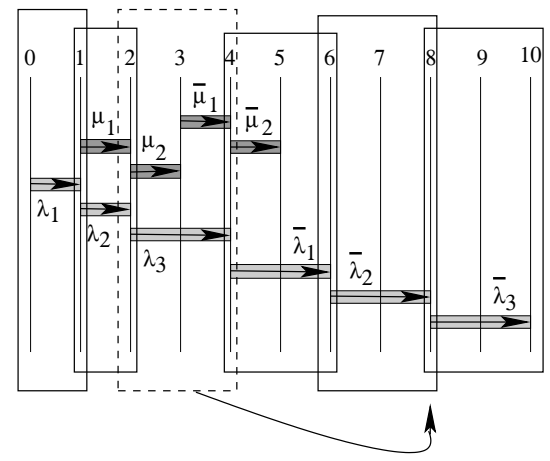

TRANSPORT A CLOSED SECTION

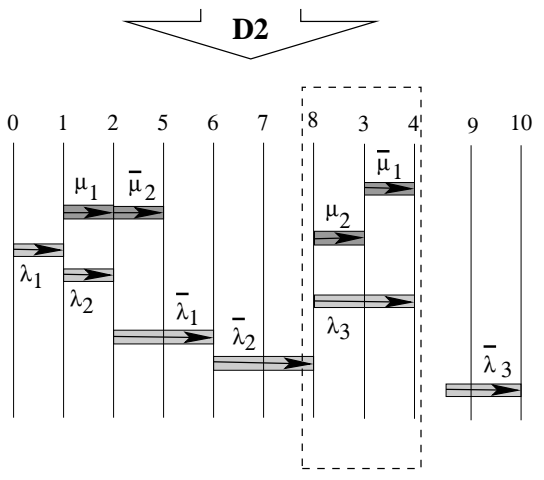

FIGURE 10. Derived transformation D2.

D3 (Complete cut).

Let $\Omega$ be a generalized equation. For every boundary connection $(p, \mu, q)$ in $\Omega$ we cut the base $\mu$ at $p$ applying ET1. The resulting generalized equation $\tilde{\Omega}$ is obtained from $\Omega$ by a consequent application of all possible ET1 transformations. Clearly, $\tilde{\Omega}$ does not depend on a particular choice of the sequence of transformations ET1. Since ET1 preserves isomorphism between the coordinate groups, equations $\Omega$ and $\tilde{\Omega}$ have isomorphic coordinate groups, and the isomorphism arises from the identity map $F[H] \rightarrow F[H]$.

D4 (Kernel of a generalized equation).

Suppose that a generalized equation $\Omega$ does not contain boundary connections. An active base $\mu \in A \Sigma_{\Omega}$ is called eliminable if at least one of the following holds:

a) $\mu$ contains an item $h_{i}$ with $\gamma\left(h_{i}\right)=1$;

b) at least one of the boundaries $\alpha(\mu), \beta(\mu)$ is different from $1, \rho+1$ and it does not touch any other base (except $\mu$ ).

A cleaning process for $\Omega$ consists of consequent removals of eliminable bases until no eliminable bases left in the equation. The resulting generalized equation is 
called a kernel of $\Omega$ and we denote it by $\operatorname{Ker}(\Omega)$. It is easy to see that $\operatorname{Ker}(\Omega)$ does not depend on a particular cleaning process. Indeed, if $\Omega$ has two different eliminable bases $\mu_{1}, \mu_{2}$, and deletion of $\mu_{i}$ results in an equation $\Omega_{i}$ then by induction (on the number of eliminations) $\operatorname{Ker}\left(\Omega_{i}\right)$ is uniquely defined for $i=1,2$. Obviously, $\mu_{1}$ is still eliminable in $\Omega_{2}$, as well as $\mu_{2}$ is eliminable in $\Omega_{1}$. Now eliminating $\mu_{1}$ and $\mu_{2}$ from $\Omega_{2}$ and $\Omega_{1}$ we get one and the same equation $\Omega_{0}$. By induction $\operatorname{Ker}\left(\Omega_{1}\right)=\operatorname{Ker}\left(\Omega_{0}\right)=\operatorname{Ker}\left(\Omega_{2}\right)$ hence the result. We say that a variable $h_{i}$ belongs to the kernel $\left(h_{i} \in \operatorname{Ker}(\Omega)\right)$, if either $h_{i}$ belongs to at least one base in the kernel, or it is parametric, or it is constant.

Also, for an equation $\Omega$ by $\bar{\Omega}$ we denote the equation which is obtained from $\Omega$ by deleting all free variables. Obviously,

$$
F_{R(\Omega)}=F_{R(\bar{\Omega})} * F(Y)
$$

where $Y$ is the set of free variables in $\Omega$.

Let us consider what happens on the group level in the cleaning process.

We start with the case when just one base is eliminated. Let $\mu$ be an eliminable base in $\Omega=\Omega\left(h_{1}, \ldots, h_{\rho}\right)$. Denote by $\Omega_{1}$ the equation resulting from $\Omega$ by eliminating $\mu$.

1) Suppose $h_{i} \in \mu$ and $\gamma\left(h_{i}\right)=1$. Then the variable $h_{i}$ occurs only once in $\Omega$ - precisely in the equation $s_{\mu}=1$ corresponding to the base $\mu$. Therefore, in the coordinate group $F_{R(\Omega)}$ the relation $s_{\mu}=1$ can be written as $h_{i}=w$, where $w$ does not contain $h_{i}$. Using Tietze transformations we can rewrite the presentation of $F_{R(\Omega)}$ as $F_{R\left(\Omega^{\prime}\right)}$, where $\Omega^{\prime}$ is obtained from $\Omega$ by deleting $s_{\mu}$ and the item $h_{i}$. It follows immediately that

$$
F_{R\left(\Omega_{1}\right)} \simeq F_{R\left(\Omega^{\prime}\right)} *\left\langle h_{i}\right\rangle
$$

and

$$
F_{R(\Omega)} \simeq F_{R\left(\Omega^{\prime}\right)} \simeq F_{R\left(\overline{\Omega_{1}}\right)} * F(Z)
$$

for some free group $F(Z)$. Notice that all the groups and equations which occur above can be found effectively.

2) Suppose now that $\mu$ satisfies case b) above with respect to a boundary $i$. Then in the equation $s_{\mu}=1$ the variable $h_{i-1}$ either occurs only once or it occurs precisely twice and in this event the second occurrence of $h_{i-1}($ in $\Delta(\mu))$ is a part of the subword $\left(h_{i-1} h_{i}\right)^{ \pm 1}$. In both cases it is easy to see that the tuple

$$
\left(h_{1}, \ldots, h_{i-2}, s_{\mu}, h_{i-1} h_{i}, h_{i+1}, \ldots, h_{\rho}\right)
$$

forms a basis of the ambient free group generated by $\left(h_{1}, \ldots, h_{\rho}\right)$ and constants from $A$. Therefore, eliminating the relation $s_{\mu}=1$, we can rewrite the presentation of $F_{R(\Omega)}$ in generators $Y=\left(h_{1}, \ldots, h_{i-2}, h_{i-1} h_{i}, h_{i+1}, \ldots, h_{\rho}\right)$. Observe also that any other equation $s_{\lambda}=1(\lambda \neq \mu)$ of $\Omega$ either does not contain variables $h_{i-1}, h_{i}$ or it contains them as parts of the subword $\left(h_{i-1} h_{i}\right)^{ \pm 1}$, i.e., any such a word $s_{\lambda}$ can be expressed as a word $w_{\lambda}(Y)$ in terms of generators $Y$ and constants from $A$. This shows that

$$
F_{R(\Omega)} \simeq F(Y \cup A)_{R\left(w_{\lambda}(Y) \mid \lambda \neq \mu\right)} \simeq F_{R\left(\Omega^{\prime}\right)},
$$

where $\Omega^{\prime}$ is a generalized equation obtained from $\Omega_{1}$ by deleting the boundary $i$. Denote by $\Omega^{\prime \prime}$ an equation obtained from $\Omega^{\prime}$ by adding a free variable $z$ to the right end of $\Omega^{\prime}$. It follows now that

$$
F_{R\left(\Omega_{1}\right)} \simeq F_{R\left(\Omega^{\prime \prime}\right)} \simeq F_{R(\Omega)} *\langle z\rangle
$$


and

$$
F_{R(\Omega)} \simeq F_{R\left(\overline{\Omega^{\prime}}\right)} * F(Z)
$$

for some free group $F(Z)$. Notice that all the groups and equations which occur above can be found effectively.

By induction on the number of steps in a cleaning process we obtain the following lemma.

LEMMA 5.2.

$$
F_{R(\Omega)} \simeq F_{R(\overline{\operatorname{Ker} \Omega})} * F(Z)
$$

where $F(Z)$ is a free group on $Z$. Moreover, all the groups and equations which occur above can be found effectively.

Proof. Let

$$
\Omega=\Omega_{0} \rightarrow \Omega_{1} \rightarrow \ldots \rightarrow \Omega_{l}=\operatorname{Ker} \Omega
$$

be a cleaning process for $\Omega$. It is easy to see (by induction on $l$ ) that for every $j=0, \ldots, l-1$

$$
\overline{\operatorname{Ker} \Omega_{j}}=\overline{\operatorname{Ker} \overline{\Omega_{j}}} .
$$

Moreover, if $\Omega_{j+1}$ is obtained from $\Omega_{j}$ as in the case 2) above, then (in the notation above)

$$
\overline{\operatorname{Ker}\left(\Omega_{j}\right)_{1}}=\overline{\operatorname{Ker} \Omega_{j}^{\prime}} .
$$

Now the statement of the lemma follows from the remarks above and equalities (20) and (21).

D5 (Entire transformation).

We need a few further definitions. A base $\mu$ of the equation $\Omega$ is called a leading base if $\alpha(\mu)=1$. A leading base is said to be maximal (or a carrier) if $\beta(\lambda) \leq \beta(\mu)$, for any other leading base $\lambda$. Let $\mu$ be a carrier base of $\Omega$. Any active base $\lambda \neq \mu$ with $\beta(\lambda) \leq \beta(\mu)$ is called a transfer base (with respect to $\mu$ ).

Suppose now that $\Omega$ is a generalized equation with $\gamma\left(h_{i}\right) \geq 2$ for each $h_{i}$ in the active part of $\Omega$. An entire transformation is a sequence of elementary transformations which are performed as follows. We fix a carrier base $\mu$ of $\Omega$. For any transfer base $\lambda$ we $\mu$-tie (applying ET5) all boundaries in $\lambda$. Using ET2 we transfer all transfer bases from $\mu$ onto $\Delta(\mu)$. Now, there exists some $i<\beta(\mu)$ such that $h_{1}, \ldots, h_{i}$ belong to only one base $\mu$, while $h_{i+1}$ belongs to at least two bases. Applying ET1 we cut $\mu$ along the boundary $i+1$. Finally, applying ET4 we delete the section $[1, i+1]$.

D6 (Identifying closed constant sections).

Let $\lambda$ and $\mu$ be two constant bases in $\Omega$ with labels $a^{\varepsilon_{\lambda}}$ and $a^{\varepsilon_{\mu}}$, where $a \in A$ and $\varepsilon_{\lambda}, \varepsilon_{\mu} \in\{1,-1\}$. Suppose that the sections $\sigma(\lambda)=[i, i+1]$ and $\sigma(\mu)=[j, j+1]$ are closed. Then we introduce a new variable base $\delta$ with its dual $\Delta(\delta)$ such that $\sigma(\delta)=[i, i+1], \sigma(\Delta(\delta))=[j, j+1], \varepsilon(\delta)=\varepsilon_{\lambda}, \varepsilon(\Delta(\delta))=\varepsilon_{\mu}$. After that we transfer all bases from $\delta$ onto $\Delta(\delta)$ using ET2, remove the bases $\delta$ and $\Delta(\delta)$, remove the item $h_{i}$, and enumerate the items in a proper order. Obviously, the coordinate group of the resulting equation is isomorphic to the coordinate group of the original equation. 
5.3. Construction of the tree $T(\Omega)$. In this section we describe a branching rewrite process for a generalized equation $\Omega$. This process results in an (infinite) tree $T(\Omega)$. At the end of the section we describe infinite paths in $T(\Omega)$.

\section{Complexity of a parametric generalized equation.}

Denote by $\rho_{A}$ the number of variables $h_{i}$ in all active sections of $\Omega$, by $n_{A}=$ $n_{A}(\Omega)$ the number of bases in active sections of $\Omega$, by $\nu^{\prime}$ - the number of open boundaries in the active sections, by $\sigma^{\prime}$ - the number of closed boundaries in the active sections.

The number of closed active sections containing no bases, precisely one base, or more than one base are denoted by $t_{A 0}, t_{A 1}, t_{A 2}$ respectively. For a closed section $\sigma \in \Sigma_{\Omega}$ denote by $n(\sigma), \rho(\sigma)$ the number of bases and, respectively, variables in $\sigma$.

$$
\begin{aligned}
& \rho_{A}=\rho_{A}(\Omega)=\sum_{\sigma \in A \Sigma_{\Omega}} \rho(\sigma) \\
& n_{A}=n_{A}(\Omega)=\sum_{\sigma \in A \Sigma_{\Omega}} n(\sigma)
\end{aligned}
$$

The complexity of a parametric equation $\Omega$ is the number

$$
\tau=\tau(\Omega)=\sum_{\sigma \in A \Sigma_{\Omega}} \max \{0, n(\sigma)-2\} .
$$

Notice that the entire transformation (D5) as well as the cleaning process (D4) do not increase complexity of equations.

Let $\Omega$ be a parametric generalized equation. We construct a tree $T(\Omega)$ (with associated structures), as a directed tree oriented from a root $v_{0}$, starting at $v_{0}$ and proceeding by induction from vertices at distance $n$ from the root to vertices at distance $n+1$ from the root.

We start with a general description of the tree $T(\Omega)$. For each vertex $v$ in $T(\Omega)$ there exists a unique generalized equation $\Omega_{v}$ associated with $v$. The initial equation $\Omega$ is associated with the root $v_{0}, \Omega_{v_{0}}=\Omega$. For each edge $v \rightarrow v^{\prime}$ (here $v$ and $v^{\prime}$ are the origin and the terminus of the edge) there exists a unique surjective homomorphism $\pi\left(v, v^{\prime}\right): F_{R\left(\Omega_{v}\right)} \rightarrow F_{R\left(\Omega_{v}^{\prime}\right)}$ associated with $v \rightarrow v^{\prime}$.

If

$$
v \rightarrow v_{1} \rightarrow \ldots \rightarrow v_{s} \rightarrow u
$$

is a path in $T(\Omega)$, then by $\pi(v, u)$ we denote composition of corresponding homomorphisms

$$
\pi(v, u)=\pi\left(v, v_{1}\right) \ldots \pi\left(v_{s}, u\right) .
$$

The set of edges of $T(\Omega)$ is subdivided into two classes: principal and auxiliary. Every newly constructed edge is principle, if not said otherwise. If $v \rightarrow v^{\prime}$ is a principle edge then there exists a finite sequence of elementary or derived transformations from $\Omega_{v}$ to $\Omega_{v^{\prime}}$ and the homomorphism $\pi\left(v, v^{\prime}\right)$ is composition of the homomorphisms corresponding to these transformations. We also assume that active [non-active] sections in $\Omega_{v^{\prime}}$ are naturally inherited from $\Omega_{v}$, if not said otherwise.

Suppose the tree $T(\Omega)$ is constructed by induction up to a level $n$, and suppose $v$ is a vertex at distance $n$ from the root $v_{0}$. We describe now how to extend the tree from $v$. The construction of the outgoing edges at $v$ depends on which case described below takes place at the vertex $v$. We always assume that if we have Case $i$, then all Cases $j$, with $j \leq i-1$, do not take place at $v$. We will see from 
the description below that there is an effective procedure to check whether or not a given case takes place at a given vertex. It will be obvious for all cases, except Case 1. We treat this case below.

\section{Preprocessing}

Case 0. In $\Omega_{v}$ we transport closed sections using D2 in such a way that all active sections are at the left end of the interval (the active part of the equation), then come all non-active sections (the non-active part of the equation), then come parametric sections (the parametric part of the equation), and behind them all constant sections are located (the constant part of the equation).

\section{Termination conditions}

Case 1. The homomorphism $\pi\left(v_{0}, v\right)$ is not an isomorphism (or equivalently, the homomorphism $\pi\left(v_{1}, v\right)$, where $v_{1}$ is the parent of $v$, is not an isomorphism). The vertex $v$ is called a leaf or an end vertex. There are no outgoing edges from $v$.

LEMMA 5.3. There is an algorithm to verify whether the homomorphism $\pi(v, u)$, associated with an edge $v \rightarrow u$ in $T(\Omega)$ is an isomorphism or not.

Proof. We will see below (by a straightforward inspection of Cases 1-15 below) that every homomorphism of the type $\pi(v, u)$ is a composition of the canonical homomorphisms corresponding to the elementary (derived) transformations. Moreover, this composition is effectively given. Now the result follows from Lemma 5.1

Case 2. $\Omega_{v}$ does not contain active sections. The vertex $v$ is called a leaf or an end vertex. There are no outgoing edges from $v$.

Moving constants to the right

Case 3. $\Omega_{v}$ contains a constant base $\delta$ in an active section such that the section $\sigma(\delta)$ is not closed.

Here we close the section $\sigma(\delta)$ using the derived transformation $D 1$.

Case 4. $\Omega_{v}$ contains a constant base $\delta$ with a label $a \in A^{ \pm 1}$ such that the section $\sigma(\delta)$ is closed.

Here we transport the section $\sigma(\delta)$ to the location right after all variables and parametric sections in $\Omega_{v}$ using the derived transformation D2. Then we identify all closed sections of the type $[i, i+1]$, which contain a constant base with the label $a^{ \pm 1}$, with the transported section $\sigma(\delta)$, using the derived transformation D6. In the resulting generalized equation $\Omega_{v^{\prime}}$ the section $\sigma(\delta)$ becomes a constant section, and the corresponding edge $\left(v, v^{\prime}\right)$ is auxiliary. See Fig. 11.

\section{Moving free variables to the right}

Case 5. $\Omega_{v}$ contains a free variable $h_{q}$ in an active section.

Here we close the section $[q, q+1]$ using D1, transport it to the very end of the interval behind all items in $\Omega_{v}$ using D2. In the resulting generalized equation $\Omega_{v^{\prime}}$ the transported section becomes a constant section, and the corresponding edge $\left(v, v^{\prime}\right)$ is auxiliary.

REMARK 5. If Cases 0-5 are not possible at $v$ then the parametric generalized equation $\Omega_{v}$ is in standard form.

Case $6 . \Omega_{v}$ contains a pair of matched bases in an active section.

Here we perform ET3 and delete it. See Fig. 12.

\section{Eliminating linear variables}

Case 7. In $\Omega_{v}$ there is $h_{i}$ in an active section with $\gamma_{i}=1$ and such that both boundaries $i$ and $i+1$ are closed. 


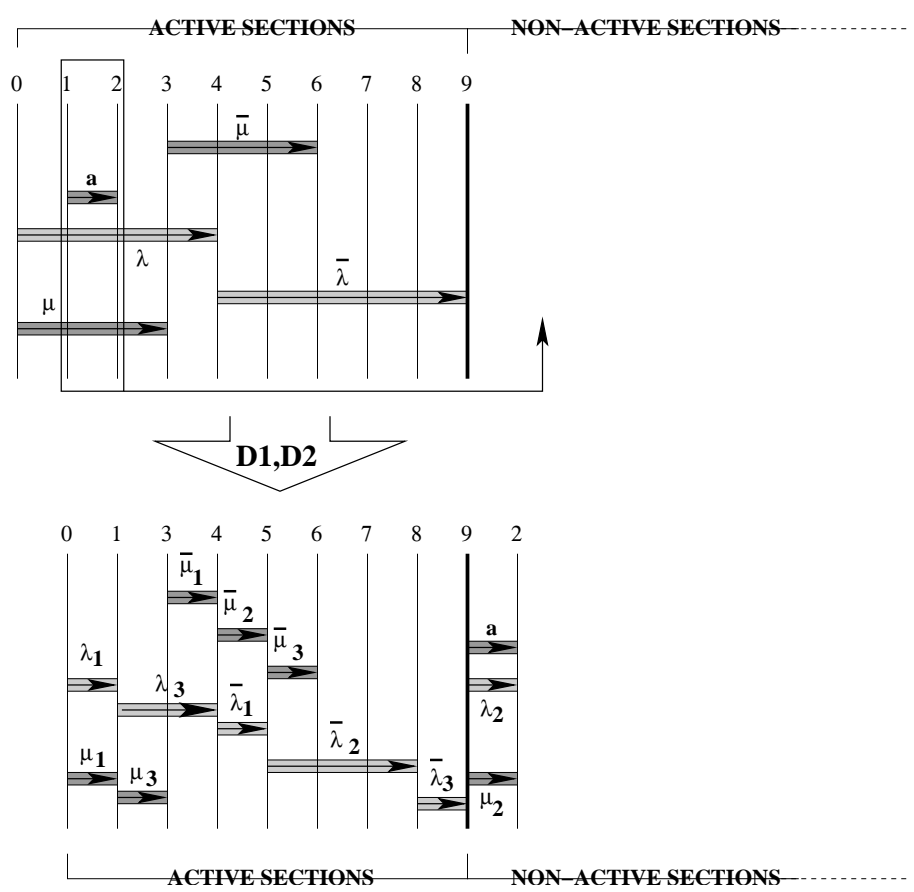

Figure 11. Case 3-4: Moving constant bases.

Here we remove the closed section $[i, i+1]$ together with the lone base using ET4.

Case 8. In $\Omega_{v}$ there is $h_{i}$ in an active section with $\gamma_{i}=1$ and such that one of the boundaries $i, i+1$ is open, say $i+1$, and the other is closed.

Here we perform ET5 and $\tau$-tie $i+1$ through the only base $\tau$ it intersects; using ET1 we cut $\tau$ in $i+1$; and then we delete the closed section $[i, i+1]$ by ET4. See Fig. 13.

Case 9. In $\Omega_{v}$ there is $h_{i}$ in an active section with $\gamma_{i}=1$ and such that both boundaries $i$ and $i+1$ are open. In addition, assume that there is a closed section $\sigma$ containing exactly two (not matched) bases $\mu_{1}$ and $\mu_{2}$, such that $\sigma=\sigma\left(\mu_{1}\right)=\sigma\left(\mu_{2}\right)$ and in the generalized equation $\tilde{\Omega}_{v}$ (see the derived transformation D3) all the bases obtained from $\mu_{1}, \mu_{2}$ by ET1 in constructing $\tilde{\Omega}_{v}$ from $\Omega_{v}$, do not belong to the kernel of $\tilde{\Omega}_{v}$.

Here, using ET5, we $\mu_{1}$-tie all the boundaries inside $\mu_{1}$; using ET2, we transfer $\mu_{2}$ onto $\Delta\left(\mu_{1}\right)$; and remove $\mu_{1}$ together with the closed section $\sigma$ using ET4.

Case 10. $\Omega_{v}$ satisfies the first assumption of Case 9 and does not satisfy the second one.

In this event we close the section $[i, i+1]$ using D1 and remove it using ET4.

Tying a free boundary

Case 11. Some boundary $i$ in the active part of $\Omega_{v}$ is free. Since we do not have Case 5 the boundary $i$ intersects at least one base, say, $\mu$.

Here we $\mu$-tie $i$ using ET5.

Quadratic case

Case 12. $\Omega_{v}$ satisfies the condition $\gamma_{i}=2$ for each $h_{i}$ in the active part. 


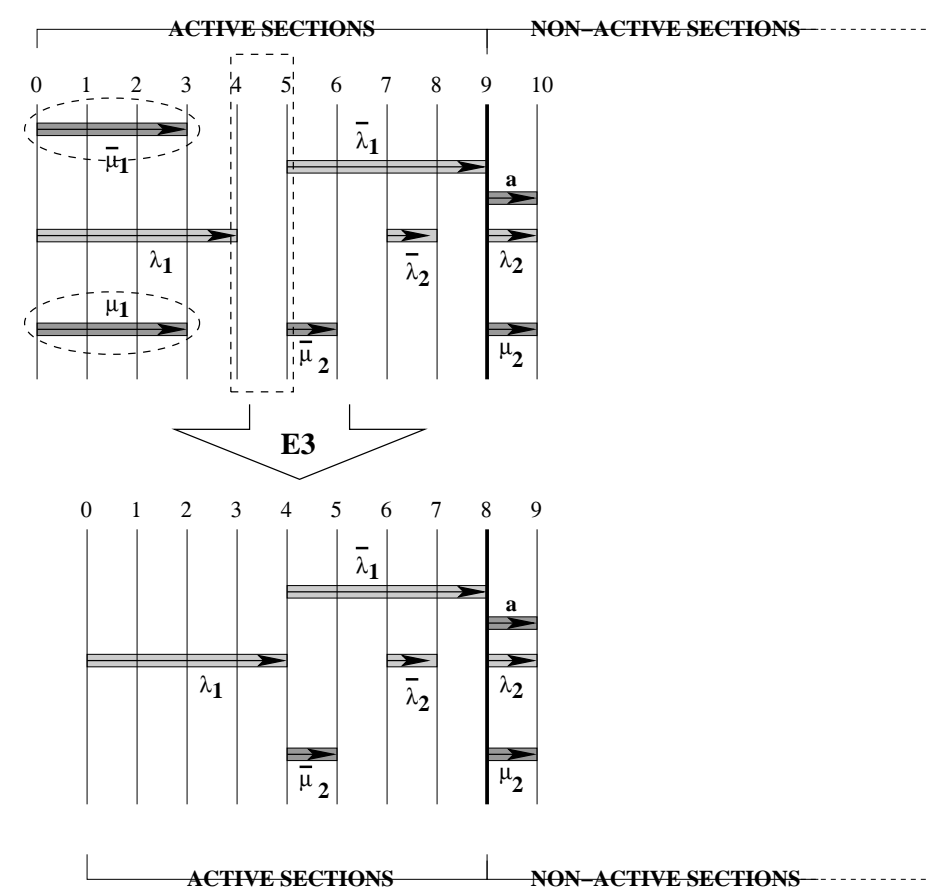

Figure 12. Case 5-6: Trivial equations and useless variables.

We apply the entire transformation D5.

Let us analyze the structure of $F_{R\left(\Omega_{v}\right)}$ in this case. Let $\sigma$ be a closed section in the active part $\sigma=\left[h_{1}, \ldots, h_{i}\right]$. Let $F_{1}$ be a free group with basis $\left\{h_{1}, \ldots, h_{i}\right\}$. If $\sigma$ contains an open boundary $j$, then we can consider a new generalized equation $\Omega_{v}^{\prime}$ obtained from $\Omega_{v}$ by replacing the product $h_{j-1} h_{j}$ by a new variable $h_{j-1}^{\prime}$ and represent $F_{R\left(\Omega_{v}\right)}=F_{R\left(\Omega_{v}^{\prime}\right)} *\left\langle h_{j}\right\rangle$. Therefore we can suppose that $\sigma$ does not contain open boundaries. We say that $\mu$ is a quadratic base if $\sigma$ contains $\mu$ and $\Delta(\mu)$, otherwise $\mu$ is a quadratic-coefficient base. Denote the set of quadratic-coefficient bases by $C$. Suppose that $\sigma$ contains quadratic bases. Let $F_{1} / \sigma$ be the quotient of $F_{1}$ over the normal closure of elements $h[\alpha(\mu), \beta(\mu)] h[\alpha(\Delta(\mu)), \beta(\Delta(\mu))]^{-1}$. Let $\mathcal{M}$ be a set that contains exactly one representative of each pair of double bases on $\sigma$ and contains also each base $\mu$ such that $\mu \in \sigma$ and $\Delta(\mu) \notin \sigma$. If we identify each base on $\sigma$ with its double, then the product $h_{1} \ldots h_{i}$ can be written as a product of bases from $\mathcal{M}$ in exactly two different ways $\mu_{i_{1}} \ldots \mu_{i_{k}}$, and $\mu_{j_{1}} \ldots \mu_{j_{t}}$. Then $F_{1} / \sigma$ is isomorphic to the quotient of the free group $F(\mathcal{M})$ over the relation $\mu_{i_{1}} \ldots \mu_{i_{k}}=\mu_{j_{1}} \ldots \mu_{j_{t}}$. Every element of $\mathcal{M}$ occurs in this relation at most twice. Applying an automorphism of $F(\mathcal{M})$ identical on $C$, we can obtain another basis $X \cup T \cup C$ of this group such that in this basis the relation has form of a standard quadratic equation in variables from $X$ with coefficients in $F(C)$ [49, Section 1.7, (variables from $T$ do not participate in any relations). The quadratic equation corresponding to $\sigma$ can be written in the standard form with coefficients expressed in terms of non-active variables. If the equation is regular, then $F_{R\left(\Omega_{v}\right)}$ has a $\mathrm{QH}$ subgroup corresponding to this equation as described in Subsection 2.9 


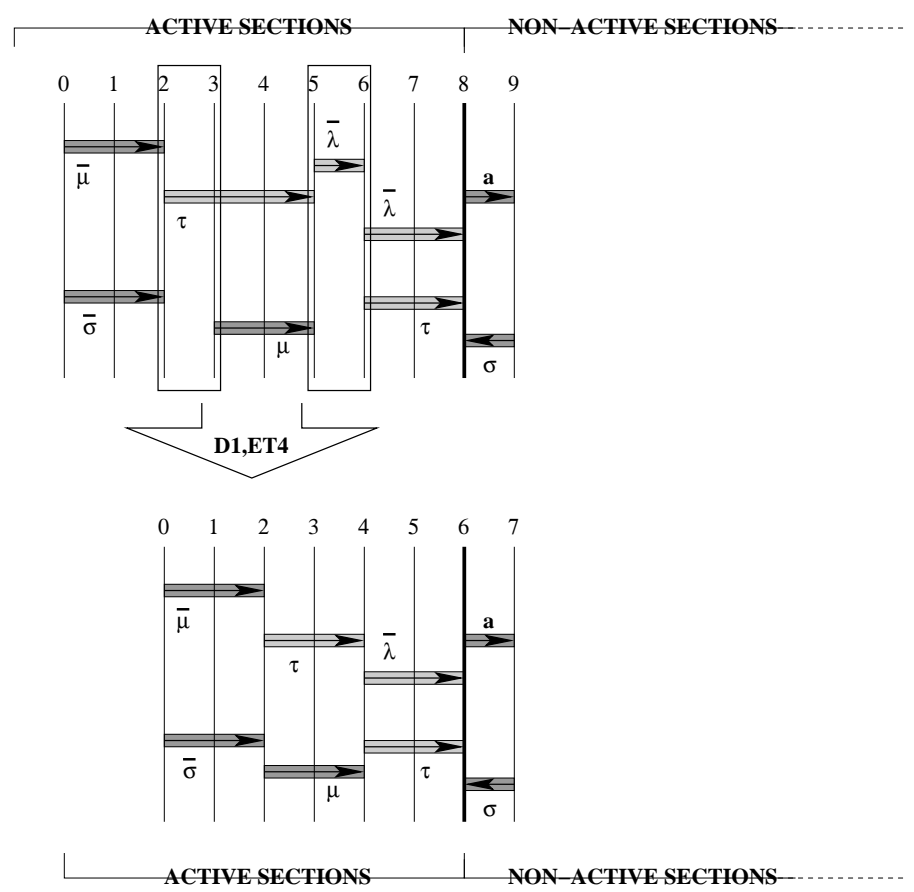

Figure 13. Case 7-10: Linear variables.

If $\sigma$ does not have quadratic-coefficient bases, then $F_{R\left(\Omega_{v}\right)}$ splits as a free product with one factor being a closed surface group.

Case 13. $\Omega_{v}$ satisfies the condition $\gamma_{i} \geq 2$ for each $h_{i}$ in the active part, and $\gamma_{i}>2$ for at least one such $h_{i}$. In addition, for some active base $\mu$ section $\sigma(\mu)=[\alpha(\mu), \beta(\mu)]$ is closed.

In this case using $E T 5$, we $\mu$-tie every boundary inside $\mu$; using $E T 2$, we transfer all bases from $\mu$ to $\Delta(\mu)$; using ET4, we remove the lone base $\mu$ together with the section $\sigma(\mu)$.

Case 14. $\Omega_{v}$ satisfies the condition $\gamma_{i} \geq 2$ for each $h_{i}$ in the active part, and $\gamma_{i}>2$ for at least one such $h_{i}$. In addition, some boundary $j$ in the active part touches some base $\lambda$, intersects some base $\mu$, and $j$ is not $\mu$-tied.

Here we $\mu$-tie $j$.

General JSJ-case

Case 15. $\Omega_{v}$ satisfies the condition $\gamma_{i} \geq 2$ for each $h_{i}$ in the active part, and $\gamma_{i}>2$ for at least one such $h_{i}$. We apply, first, the entire transformation D5.

Here for every boundary $j$ in the active part that touches at least one base, we $\mu$-tie $j$ by every base $\mu$ containing $j$. This results in finitely many new vertices $\Omega_{v^{\prime}}$ with principle edges $\left(v, v^{\prime}\right)$.

If, in addition, $\Omega_{v}$ satisfies the following condition (we called it Case 15.1 in [36]) then we construct the principle edges as was described above, and also construct a few more auxiliary edges outgoing from the vertex $v$ :

Case 15.1. The carrier base $\mu$ of the equation $\Omega_{v}$ intersects with its dual $\Delta(\mu)$.

Here we construct an auxiliary equation $\hat{\Omega}_{v}$ (which does not occur in $T(\Omega)$ ) as follows. Firstly, we add a new constant section $\left[\rho_{v}+1, \rho_{v}+2\right]$ to the right of all 
sections in $\Omega_{v}$ (in particular, $h_{\rho_{v}+1}$ is a new free variable). Secondly, we introduce a new pair of bases $(\lambda, \Delta(\lambda))$ such that

$$
\alpha(\lambda)=1, \beta(\lambda)=\beta(\Delta(\mu)), \alpha(\Delta(\lambda))=\rho_{v}+1, \beta(\Delta(\lambda))=\rho_{v}+2 .
$$

Notice that $\Omega_{v}$ can be obtained from $\hat{\Omega}_{v}$ by ET4: deleting $\delta(\lambda)$ together with the closed section $\left[\rho_{v}+1, \rho_{v}+2\right]$. Let

$$
\hat{\pi}_{v}: F_{R\left(\Omega_{v}\right)} \rightarrow F_{R\left(\hat{\Omega}_{v}\right)}
$$

be the isomorphism induced by ET4. Case 15 still holds for $\hat{\Omega}_{v}$, but now $\lambda$ is the carrier base. Applying to $\hat{\Omega}_{v}$ transformations described in Case 15 , we obtain a list of new vertices $\Omega_{v^{\prime}}$ together with isomorphisms

$$
\eta_{v^{\prime}}: F_{R\left(\hat{\Omega}_{v}\right)} \rightarrow F_{R\left(\Omega_{v^{\prime}}\right)} .
$$

Now for each such $v^{\prime}$ we add to $T(\Omega)$ an auxiliary edge $\left(v, v^{\prime}\right)$ equipped with composition of homomorphisms $\pi\left(v, v^{\prime}\right)=\eta_{v^{\prime}} \circ \hat{\pi}_{v}$ and assign $\Omega_{v^{\prime}}$ to the vertex $v^{\prime}$.

If none of the Cases 0-15 is possible, then we stop, and the tree $T(\Omega)$ is constructed. In any case, the tree $T(\Omega)$ is constructed by induction. Observe that, in general, $T(\Omega)$ is an infinite locally finite tree.

If Case $i(0 \leq i \leq 15)$ takes place at a vertex $v$ then we say that $v$ has type $i$ and write $t p(v)=i$.

Example. Consider the generalized equation in Fig. 3. For this generalized equation we first have to move constants to the right (Cases 3, 4). After this we have Case 11 (tieing free boundaries). Then we have Case 12 (the entire transformation). At the beginning $\lambda_{1}$ is the leading base, then $\mu$ is the leading base. After these transformations we obtain the generalized equation in Fig. 14.

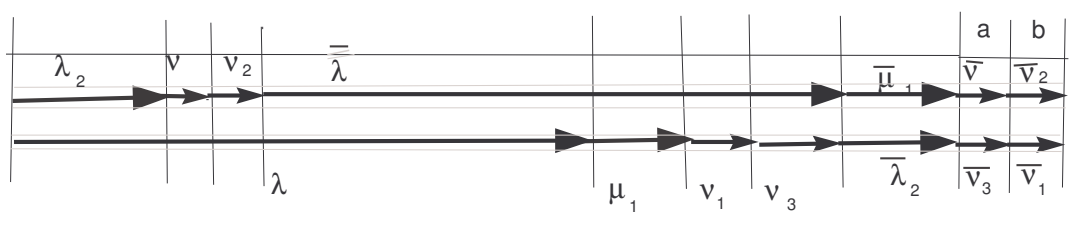

$[x, y][b, a]=1$

Figure 14. Case 12 for the generalized equation $\Omega$ for the equation $[x, y][b, a]=1$. 
For this equation we still have Case 12. Beginning at this vertex of the tree $T(\Omega)$ we have an infinite branch such that for all the vertices $v_{i}$ of this branch $\Omega_{v_{i}}=\Omega_{v_{i+1}}$.

LEMMA 5.4. (Lemma 3.1, 62 ) If $u \rightarrow v$ is a principal edge of the tree $T(\Omega)$, then

(1) $n_{A}\left(\Omega_{v}\right) \leq n_{A}\left(\Omega_{u}\right)$, if $\operatorname{tp}(u) \neq 3,10$, this inequality is proper if $t p(u)=$ $6,7,9,13$

(2) If $\operatorname{tp}(u)=10$, then $n_{A}\left(\Omega_{v}\right) \leq n_{A}\left(\Omega_{u}\right)+2$;

(3) $\nu^{\prime}\left(\Omega_{v}\right) \leq \nu^{\prime}\left(\Omega_{u}\right)$ if $t p(u) \leq 13$ and $t p(u) \neq 3,11$;

(4) $\tau\left(\Omega_{v}\right) \leq \tau\left(\Omega_{u}\right)$, if $\operatorname{tp}(u) \neq 3$.

PROOF. Straightforward verification.

LEMMA 5.5. Let

$$
v_{1} \rightarrow v_{2} \rightarrow \ldots \rightarrow v_{r} \rightarrow \ldots
$$

be an infinite path in the tree $T(\Omega)$. Then there exists a natural number $N$ such that all the edges $v_{n} \rightarrow v_{n+1}$ of this path with $n \geq N$ are principal edges, and one of the following situations holds:

1) (linear case) $7 \leq t p\left(v_{n}\right) \leq 10$ for all $n \geq N$;

2) (quadratic case) $t p\left(v_{n}\right)=12$ for all $n \geq N$;

3) (general JSJ case) $t p\left(v_{n}\right)=15$ for all $n \geq N$.

Proof. Observe that starting with a generalized equation $\Omega$ we can have Case 0 only once, afterward in all other equations the active part is at the left, then comes the non-active part, then - the parametric part, and at the end - the constant part. Obviously, Cases 1 and 2 do not occur on an infinite path. Notice also that Cases 3 and 4 can only occur finitely many times, namely, not more then $2 t$ times where $t$ is the number of constant bases in the original equation $\Omega$. Therefore, there exists a natural number $N_{1}$ such that $\operatorname{tp}\left(v_{i}\right) \geq 5$ for all $i \geq N_{1}$.

Now we show that the number of vertices $v_{i}(i \geq N)$ for which $t p\left(v_{i}\right)=5$ is not more than the minimal number of generators of the group $F_{R(\Omega)}$, in particular, it cannot be greater than $\rho+1+|A|$, where $\rho=\rho(\Omega)$. Indeed, if a path from the root $v_{0}$ to a vertex $v$ contains $k$ vertices of type 5 , then $\Omega_{v}$ has at least $k$ free variables in the constant part. This implies that the coordinate group $F_{R\left(\Omega_{v}\right)}$ has a free group of rank $k$ as a free factor, hence it cannot be generated by less than $k$ elements. Since $\pi\left(v_{0}, v\right): F_{R(\Omega)} \rightarrow F_{R\left(\Omega_{v}\right)}$ is a surjective homomorphism, the group $F_{R(\Omega)}$ cannot be generated by less then $k$ elements. This shows that $k \leq \rho+1+|A|$. It follows that there exists a number $N_{2} \geq N_{1}$ such that $\operatorname{tp}\left(v_{i}\right)>5$ for every $i \geq N_{2}$.

Suppose $i>N_{2}$. If $\operatorname{tp}\left(v_{i}\right)=12$, then it is easy to see that $t p\left(v_{i+1}\right)=6$ or $t p\left(v_{i+1}\right)=12$. But if $t p\left(v_{i+1}\right)=6$, then $t p\left(v_{i+2}\right)=5$ - contradiction with $i>N_{2}$. Therefore, $\operatorname{tp}\left(v_{i+1}\right)=t p\left(v_{i+2}\right)=\ldots=t p\left(v_{i+j}\right)=12$ for every $j>0$ and we have situation 2) of the lemma.

Suppose now $t p\left(v_{i}\right) \neq 12$ for all $i \geq N_{2}$. By Lemma $5.4 \tau\left(\Omega_{v_{j+1}}\right) \leq \tau\left(\Omega_{v_{j}}\right)$ for every principle edge $v_{j} \rightarrow v_{j+1}$ where $j \geq N_{2}$. If $v_{j} \rightarrow v_{j+1}$, where $j \geq N_{2}$, is an auxiliary edge then $t p\left(v_{j}\right)=15$ and, in fact, Case 15.1 takes place at $v_{j}$. In the notation of Case $15.1 \Omega_{v_{j+1}}$ is obtained from $\hat{\Omega}_{v_{j}}$ by transformations from Case 15. In this event, both bases $\mu$ and $\Delta(\mu)$ will be transferred from the new carrier base $\lambda$ to the constant part, so the complexity will be decreased at least 
by two: $\tau\left(\Omega_{v_{j+1}}\right) \leq \tau\left(\hat{\Omega}_{v_{j}}\right)-2$. Observe also that $\tau\left(\hat{\Omega}_{v_{j}}\right)=\tau\left(\Omega_{v_{j}}\right)+1$. Hence $\tau\left(\Omega_{v_{j+1}}\right)<\tau\left(\Omega_{v_{j}}\right)$.

It follows that there exists a number $N_{3} \geq N_{2}$ such that $\tau\left(\Omega_{v_{j}}\right)=\tau\left(\Omega_{v_{N_{3}}}\right)$ for every $j \geq N_{3}$, i.e., complexity stabilizes. Since every auxiliary edge gives a decrease of complexity, this implies that for every $j \geq N_{3}$ the edge $v_{j} \rightarrow v_{j+1}$ is principle.

Suppose now that $i \geq N_{3}$. We claim that $t p\left(v_{i}\right) \neq 6$. Indeed, if $t p\left(v_{i}\right)=6$, then the closed section, containing the matched bases $\mu, \Delta(\mu)$, does not contain any other bases (otherwise the complexity of $\Omega_{v_{i+1}}$ would decrease). But in this event $t p\left(v_{i+1}\right)=5$ which is impossible.

So $t p\left(v_{i}\right) \geq 7$ for every $i \geq N_{3}$. Observe that ET3 (deleting match bases) is the only elementary transformation that can produce new free boundaries. Observe also that ET3 can be applied only in Case 6 . Since Case 6 does not occur anymore along the path for $i \geq N_{3}$, one can see that no new free boundaries occur in equations $\Omega_{v_{j}}$ for $j \geq N_{3}$. It follows that there exists a number $N_{4} \geq N_{3}$ such that $\operatorname{tp}\left(v_{i}\right) \neq 11$ for every $j \geq N_{4}$.

Suppose now that for some $i \geq N_{4} 13 \leq t p\left(v_{i}\right) \leq 15$. It is easy to see from the description of these cases that in this event $t p\left(v_{i+1}\right) \in\{6,13,14,15\}$. Since $\operatorname{tp}\left(v_{i+1}\right) \neq 6$, this implies that $13 \leq t p\left(v_{j}\right) \leq 15$ for every $j \geq i$. In this case the sequence $n_{A}\left(\Omega_{v_{j}}\right)$ stabilizes by lemma 5.4 In addition, if $t p\left(v_{j}\right)=13$, then $n_{A}\left(\Omega_{v_{j+1}}\right)<n_{A}\left(\Omega_{v_{j}}\right)$. Hence there exists a number $N_{5} \geq N_{4}$ such that $t p\left(v_{j}\right) \neq 13$ for all $j \geq N_{5}$.

Suppose $i \geq N_{5}$. There cannot be more than $8\left(n_{A}\left(\Omega_{v_{i}}\right)^{2}\right.$ vertices of type $14 \mathrm{in}$ a row starting at a vertex $v_{i}$; hence there exists $j \geq i$ such that $t p\left(v_{j}\right)=15$. The series of transformations ET5 in Case 15 guarantees the inequality $t p\left(v_{j+1}\right) \neq 14$; hence $t p\left(v_{j+1}\right)=15$, and we have situation 3) of the lemma.

So we can suppose $t p\left(v_{i}\right) \leq 10$ for all the vertices of our path. Then we have situation 1) of the lemma.

\section{Elimination process: periodic structures}

Recall that a reduced word $P$ in a free group $F$ is called a period if it is cyclically reduced and not a proper power. A word $w \in F$ is called $P$-periodic if $d(w) \geq d(P)$ and it is a subword of some (integer) power of $P$. Every $P$-periodic word $w$ can be presented in the form

$$
w=A^{r} A_{1}
$$

where $A$ is a cyclic permutation of $P^{ \pm 1}, r \geq 1, A=A_{1} \circ A_{2}$, and $A_{2} \neq 1$. This representation is unique if $r \geq 2$. The number $r$ is called the exponent of $w$. A maximal exponent of $P$-periodic subword in $u$ is called the exponent of $P$-periodicity in $u$. We denote it $e_{P}(u)$.

Definition 6.1. Let $\Omega$ be a standard generalized equation. A solution $H$ : $h_{i} \rightarrow H_{i}$ of $\Omega$ is called periodic with respect to a period $P$, if for every variable section $\sigma$ of $\Omega$ one of the following conditions hold:

1) $H(\sigma)$ is $P$-periodic with exponent $r \geq 2$;

2) $d(H(\sigma)) \leq d(P)$

3) $H(\sigma)$ is $A$-periodic and $d(A) \leq d(P)$;

Moreover, condition 1) holds at least for one such $\sigma$. 
The section $\sigma$ is called $P$-periodic. In this section we will prove the following result.

Proposition 6.2. 1. Let $\Omega$ be a generalized equation. One can effectively find a number $N$ such that every periodic solution of $\Omega$ with some period $P$ (see Definition [6.1) is a composition of a canonical automorphism of the coordinate group $F_{R(\Omega)}$ with either a solution with exponent of periodicity for $P$ less than $N$ or a solution of a fixed equation not in $R(\Omega)$. These canonical automorphisms correspond to Dehn twists of $F_{R(\Omega)}$ which are related to the splitting of $F_{R(\Omega)}$ (which comes from a so-called periodic structure) over an abelian edge group.

2. Let $\Omega$ be a generalized equation with parameters $Y$ that has a periodic solution $H$ of $\Omega$ with period $P$. Then one can effectively find a number $N$ such that there are two possibilities.

(1) $F_{R(\Omega)}$ has a splitting modulo $\langle Y\rangle$ and $H$ is a composition of a canonical automorphism of the coordinate group $F_{R(\Omega)}$ corresponding to this splitting (fixing the subgroup of parameters) with a solution with exponent of $P$ bounded by $N e(H(Y))$ or a solution of an equation not in $R(\Omega)$.

(2) $e(H) \leq N e(H(Y))$.

One can effectively find $N$ and the splitting.

We will prove this proposition in Subsection 6.4 We will later formulate a more detailed statement about splittings of $F_{R(\Omega)}$ (see Theorem [7.1).

6.1. Definition of a periodic structure. In this subsection we introduce a notion of a periodic structure which allows one to describe periodic solutions of generalized equations.

We fix till the end of the section a generalized equation $\Omega$ in standard form. Recall that in $\Omega$ all closed sections $\sigma$, bases $\mu$, and variables $h_{i}$ belong to either the variable part $V \Sigma$, or the parametric part $P \Sigma$, or the constant part $C \Sigma$ of $\Omega$.

DeFinition 6.3. Let $\Omega$ be a generalized equation in standard form with no boundary connections. A periodic structure on $\Omega$ is a pair $\langle\mathcal{P}, R\rangle$, where

1) $\mathcal{P}$ is a set consisting of some variables $h_{i}$, some bases $\mu$, and some closed sections $\sigma$ from $V \Sigma$ and such that the following conditions are satisfied:

a) if $h_{i} \in \mathcal{P}$ and $h_{i} \in \mu$, and $\Delta(\mu) \in V \Sigma$, then $\mu \in \mathcal{P}$;

b) if $\mu \in \mathcal{P}$, then $\Delta(\mu) \in \mathcal{P}$;

c) if $\mu \in \mathcal{P}$ and $\mu \in \sigma$, then $\sigma \in \mathcal{P}$;

d) there exists a function $\mathcal{X}$ mapping the set of closed sections from $\mathcal{P}$ into $\{-1,+1\}$ such that for every $\mu, \sigma_{1}, \sigma_{2} \in \mathcal{P}$, the condition that $\mu \in \sigma_{1}$ and $\Delta(\mu) \in \sigma_{2}$ implies $\varepsilon(\mu) \cdot \varepsilon(\Delta(\mu))=\mathcal{X}\left(\sigma_{1}\right) \cdot \mathcal{X}\left(\sigma_{2}\right) ;$

2) $R$ is an equivalence relation on a certain set $\mathcal{B}$ (defined below) such that the following conditions are satisfied:

e) Notice, that for every boundary $l$ either there exists a unique closed section $\sigma(l)$ in $\mathcal{P}$ containing $l$, or there exist precisely two closed section $\sigma_{\text {left }}(l)=[i, l], \sigma_{\text {right }}=[l, j]$ in $\mathcal{P}$ containing $l$. The set of boundaries of the first type we denote by $\mathcal{B}_{1}$, and of the second type - by $\mathcal{B}_{2}$. Put

$$
\mathcal{B}=\mathcal{B}_{1} \cup\left\{l_{\text {left }}, l_{\text {right }} \mid l \in \mathcal{B}_{2}\right\}
$$


here $l_{\text {left }}, l_{\text {right }}$ are two "formal copies" of $l$. We will use the following agreement: for any base $\mu$ if $\alpha(\mu) \in \mathcal{B}_{2}$ then by $\alpha(\mu)$ we mean $\alpha(\mu)_{\text {right }}$ and, similarly, if $\beta(\mu) \in \mathcal{B}_{2}$ then by $\beta(\mu)$ we mean $\beta(\mu)_{\text {left }}$. f) Now, we define $R$ as follows. If $\mu \in \mathcal{P}$ then

$$
\begin{gathered}
\alpha(\mu) \sim_{R} \alpha(\Delta(\mu)), \quad \beta(\mu), \sim_{R} \beta(\Delta(\mu)) \quad \text { if } \varepsilon(\mu)=\varepsilon(\Delta(\mu)) \\
\alpha(\mu)) \sim_{R} \beta(\Delta(\mu)), \beta(\mu) \sim_{R} \alpha(\Delta(\mu)) \text { if } \varepsilon(\mu)=-\varepsilon(\Delta(\mu)) .
\end{gathered}
$$

REMARK 6. For a given $\Omega$ one can effectively find all periodic structures on $\Omega$.

Let $\langle\mathcal{P}, R\rangle$ be a periodic structure of $\Omega$. Put

$\mathcal{N} P=\left\{\mu \in B \Omega \mid \exists h_{i} \in \mathcal{P}\right.$ such that $h_{i} \in \mu$ and $\Delta(\mu)$ is parametric or constant $\}$

Now we will show how one can associate with a $P$-periodic solution $H$ of $\Omega$ a periodic structure $\mathcal{P}(H, P)=\langle\mathcal{P}, R\rangle$. We define $\mathcal{P}$ as follows. A closed section $\sigma$ is in $\mathcal{P}$ if and only if $\sigma \in V \Sigma$ and $H(\sigma)$ is $P$-periodic with exponent $\geq 2$. A variable $h_{i}$ is in $\mathcal{P}$ if and only if $h_{i} \in \sigma$ for some $\sigma \in \mathcal{P}$ and $d\left(H_{i}\right) \geq 2 d(P)$. A base $\mu$ is in $\mathcal{P}$ if and only if both $\mu$ and $\Delta(\mu)$ are in $v \Sigma$ and one of them contains $h_{i}$ from $\mathcal{P}$.

Lemma 6.4. Let $H$ be a periodic solution of $\Omega$. Then $\mathcal{P}(H, P)$ is a periodic structure on $\Omega$.

Proof. Let $\mathcal{P}(H, P)=\langle\mathcal{P}, R\rangle$. Obviously, $\mathcal{P}$ satisfies a) and b) from the definition 6.3

Let $\mu \in \mathcal{P}$ and $\mu \in[i, j]$. There exists an unknown $h_{k} \in \mathcal{P}$ such that $h_{k} \in \mu$ or $h_{k} \in \Delta(\mu)$. If $h_{k} \in \mu$, then, obviously, $[i, j] \in \mathcal{P}$. If $h_{k} \in \Delta(\mu)$ and $\Delta(\mu) \in\left[i^{\prime}, j^{\prime}\right]$, then $\left[i^{\prime}, j^{\prime}\right] \in \mathcal{P}$, and hence, the word $H[\alpha(\Delta(\mu)), \beta(\Delta(\mu))]$ can be written in the form $Q^{r^{\prime}} Q_{1}$, where $Q=Q_{1} Q_{2} ; Q$ is a cyclic shift of the word $P^{ \pm 1}$ and $r^{\prime} \geq 2$. Now let (22) be a presentation for the section $[i, j]$. Then $H[\alpha(\mu), \beta(\mu)]=B^{s} B_{1}$, where $B$ is a cyclic shift of the word $A^{ \pm 1}, d(B) \leq d(P), B=B_{1} B_{2}$, and $s \geq 0$. From the equality $\left.H[\alpha(\mu), \beta(\mu)]^{\varepsilon(\mu)}=H[\alpha(\Delta(\mu)), \beta(\Delta(\mu)))\right]^{\varepsilon(\Delta(\mu))}$ and Lemma 1.2.9 [1] it follows that $B$ is a cyclic shift of the word $Q^{ \pm 1}$. Consequently, $A$ is a cyclic shift of the word $P^{ \pm 1}$, and $r \geq 2$ in (22), since $d(H[i, j]) \geq d(H[\alpha(\mu), \beta(\mu)]) \geq 2 d(P)$. Therefore, $[i, j] \in \mathcal{P}$; i.e, part c) of the definition 6.3 holds.

Put $\mathcal{X}([i, j])= \pm 1$ depending on whether in (22) the word $A$ is conjugate to $P$ or to $P^{-1}$. If $\mu \in\left[i_{1}, j_{1}\right], \Delta(\mu) \in\left[i_{2}, j_{2}\right]$, and $\mu \in \mathcal{P}$, then the equality $\varepsilon(\mu) \cdot \varepsilon(\Delta(\mu))$ $=\mathcal{X}\left(\left[i_{1}, j_{1}\right]\right) \cdot \mathcal{X}\left(\left[i_{2}, j_{2}\right]\right)$ follows from the fact that given $A^{r} A_{1}=B^{s} B_{1}$ and $r, s \geq 2$, the word $A$ cannot be a cyclic shift of the word $B^{-1}$. Hence part d) also holds.

Now let $[i, j] \in \mathcal{P}$ and $i \leq l \leq j$. Then there exists a subdivision $P=P_{1} P_{2}$ such that if $\mathcal{X}([i, j])=1$, then the word $H[i, l]$ is the end of the word $\left(P^{\infty}\right) P_{1}$, where $P^{\infty}$ is the infinite word obtained by concatenations of powers of $P$, and $H[l, j]$ is the beginning of the word $P_{2}\left(P^{\infty}\right)$, and if $\mathcal{X}([i, j])=-1$, then the word $H[i, l]$ is the end of the word $\left(P^{-1}\right)^{\infty} P_{2}^{-1}$ and $H[l, j]$ is the beginning of $P_{1}^{-1}\left(P^{-1}\right)^{\infty}$. Again, Lemma 1.2.9 [1] implies that the subdivision $P=P_{1} P_{2}$ with the indicated properties is unique; denote it by $\delta(l)$. Let us define a relation $R$ in the following way: $R\left(l_{1}, l_{2}\right) \rightleftharpoons \delta\left(l_{1}\right)=\delta\left(l_{2}\right)$. Condition e) of the definition of a periodic structure obviously holds.

Condition f) follows from the graphic equality $H[\alpha(\mu), \beta(\mu)]^{\varepsilon(\mu)}=H[\alpha(\Delta(\mu))$, $\beta(\Delta(\mu))]^{\varepsilon(\Delta(\mu))}$ and Lemma 1.2.9 [1].

This proves the lemma. 
Now let us fix a nonempty periodic structure $\langle\mathcal{P}, R\rangle$. Item d) allows us to assume (after replacing the variables $h_{i}, \ldots, h_{j-1}$ by $h_{j-1}^{-1}, \ldots, h_{i}^{-1}$ on those sections $[i, j] \in \mathcal{P}$ for which $\mathcal{X}([i, j])=-1)$ that $\varepsilon(\mu)=1$ for all $\mu \in \mathcal{P}$. For a boundary $k$, we will denote by $(k)$ the equivalence class of the relation $R$ to which it belongs.

Let us construct an oriented graph $\Gamma$ whose set of vertices is the set of $R$ equivalence classes. For each unknown $h_{k}$ lying on a certain closed section from $\mathcal{P}$, we introduce an oriented edge $e$ leading from $(k)$ to $(k+1)$ and an inverse edge $e^{-1}$ leading from $(k+1)$ to $(k)$. This edge $e$ is assigned the label $h(e) \rightleftharpoons h_{k}$ (respectively, $h\left(e^{-1}\right) \rightleftharpoons h_{k}^{-1}$.) For every path $r=e_{1}^{ \pm 1} \ldots e_{s}^{ \pm 1}$ in the graph $\Gamma$, we denote by $h(r)$ its label $h\left(e_{1}^{ \pm 1}\right) \ldots h\left(e_{j}^{ \pm 1}\right)$. The periodic structure $\langle\mathcal{P}, R\rangle$ is called connected, if the graph $\Gamma$ is connected. Suppose first that $\langle\mathcal{P}, R\rangle$ is connected. We can always suppose that each boundary of $\Omega$ is a boundary between two bases.

Lemma 6.5. Let $H$ be a P-periodic solution of a generalized equation $\Omega,\langle\mathcal{P}, R\rangle=$ $\mathcal{P}(H, P) ; c$ be a cycle in the graph $\Gamma$ at the vertex $(l) ; \delta(l)=P_{1} P_{2}$. Then there exists $n \in \mathbb{Z}$ such that $H(c)=\left(P_{2} P_{1}\right)^{n}$.

Proof. If $e$ is an edge in the graph $\Gamma$ with initial vertex $V^{\prime}$ and terminal vertex $V^{\prime \prime}$, and $P=P_{1}^{\prime} P_{2}^{\prime}, P=P_{1}^{\prime \prime} P_{2}^{\prime \prime}$ are two subdivisions corresponding to the boundaries from $V^{\prime}, V^{\prime \prime}$ respectively, then, obviously, $H(e)=P_{2}^{\prime} P^{n_{k}} P_{1}^{\prime \prime}\left(n_{k} \in \mathbb{Z}\right)$. The claim is easily proven by multiplying together the values $H(E)$ for all the edges $e$ taking part in the cycle $c$.

DeFinition 6.6. A generalized equation $\Omega$ is called periodized with respect to a given periodic structure $\langle\mathcal{P}, R\rangle$ of $\Omega$, if for every two cycles $c_{1}$ and $c_{2}$ with the same initial vertex in the graph $\Gamma$, there is a relation $\left[h\left(c_{1}\right), h\left(c_{2}\right)\right]=1$ in $F_{R(\Omega)}$.

6.2. Case 1. Set $N \mathcal{P}$ is empty. Let $\Gamma_{0}$ be the subgraph of the graph $\Gamma$ having the same set of vertices and consisting of the edges $e$ whose labels do not belong to $\mathcal{P}$. Choose a maximal subforest $T_{0}$ in the graph $\Gamma_{0}$ and extend it to a maximal subforest $T$ of the graph $\Gamma$. Since $\langle\mathcal{P}, R\rangle$ is connected by assumption, it follows that $T$ is a tree. Let $V_{0}$ be an arbitrary vertex of the graph $\Gamma$ and $r\left(V_{0}, V\right)$ the (unique) path from $V_{0}$ to $V$ all of whose vertices belong to $T$. For every edge $e: V \rightarrow V^{\prime}$ not lying in $T$, we introduce a cycle $c_{e}=r\left(V_{0}, V\right) e\left(r\left(V_{0}, V^{\prime}\right)\right)^{-1}$. Then the fundamental group $\pi_{1}\left(\Gamma, V_{0}\right)$ is generated by the cycles $c_{e}$ (see, for example, the proof of Proposition 3.2.1 [49]). This and decidability of the universal theory of a free group imply that the property of a generalized equation "to be periodized with respect to a given periodic structure" is algorithmically decidable.

Furthermore, the set of elements

$$
\{h(e) \mid e \in T\} \cup\left\{h\left(c_{e}\right) \mid e \notin T\right\}
$$

forms a basis of the free group with the set of generators $\left\{h_{k} \mid h_{k}\right.$ is an unknown lying on a closed section from $\mathcal{P}\}$. If $\mu \in \mathcal{P}$, then $(\beta(\mu))=(\beta(\Delta(\mu)))$, $(\alpha(\mu))=(\alpha(\Delta(\mu)))$ by part f) from Definition 6.3 and, consequently, the word $h[\alpha(\mu), \beta(\mu)] h[\alpha(\Delta(\mu)), \beta(\Delta(\mu))]^{-1}$ is the label of a cycle $c^{\prime}(\mu)$ from $\pi_{1}(\Gamma,(\alpha(\mu)))$. Let $c(\mu)=r\left(V_{0},(\alpha(\mu))\right) c^{\prime}(\mu) r\left(V_{0},(\alpha(\mu))\right)^{-1}$. Then

$$
h(c(\mu))=u h[\alpha(\mu), \beta(\mu)] h[\alpha(\Delta(\mu)), \beta(\Delta(\mu))]^{-1} u^{-1},
$$


where $u$ is a certain word. Since $c(\mu) \in \pi_{1}\left(\Gamma, V_{0}\right)$, it follows that $c(\mu)=b_{\mu}\left(\left\{c_{e} \mid e \notin\right.\right.$ $T\}$ ), where $b_{\mu}$ is a certain word in the indicated generators which can be effectively constructed (see Proposition 3.2.1 [49]).

Let $\tilde{b}_{\mu}$ denote the image of the word $b_{\mu}$ in the factor group of $\pi\left(\Gamma, V_{0}\right)$ over the derived subgroup. Denote by $\widetilde{Z}$ the free abelian group consisting of formal linear combinations $\sum_{e \notin T} n_{e} \tilde{c}_{e}\left(n_{e} \in \mathbb{Z}\right)$, and by $\widetilde{B}$ its subgroup generated by the elements $\tilde{b}_{\mu}(\mu \in \mathcal{P})$ and the elements $\tilde{c}_{e}(e \notin T, h(e) \notin \mathcal{P})$. Let $\widetilde{A}=\widetilde{Z} / \widetilde{B}, T(\widetilde{A})$ the torsion subgroups of the group $\widetilde{A}$, and $\widetilde{Z}_{1}$ the preimage of $T(\widetilde{A})$ in $\widetilde{Z}$. The group $\widetilde{Z} / \widetilde{Z}_{1}$ is free; therefore, there exists a decomposition of the form

$$
\widetilde{Z}=\widetilde{Z}_{1} \oplus \widetilde{Z}_{2}, B \subseteq \widetilde{Z}_{1},\left(\widetilde{Z}_{1}: \widetilde{B}\right)<\infty .
$$

Note that it is possible to express effectively a certain basis $\widetilde{\bar{c}}^{(1)}, \widetilde{\bar{c}}^{(2)}$ of the group $\widetilde{Z}$ in terms of the generators $\widetilde{c}_{e}$ so that for the subgroups $\widetilde{Z}_{1}, \widetilde{Z}_{2}$ generated by the sets $\widetilde{\bar{c}}^{(1)}, \widetilde{\bar{c}}^{(2)}$ respectively, relation 250 holds. For this it suffices, for instance, to look through the bases one by one, using the fact that under the condition $\widetilde{Z}=\widetilde{Z}_{1} \oplus \widetilde{Z}_{2}$ the relations $\widetilde{B} \subseteq \widetilde{Z}_{1},\left(\widetilde{Z}_{1}: \widetilde{B}\right)<\infty$ hold if and only if the generators of the groups $\widetilde{B}$ and $\widetilde{Z}_{1}$ generate the same linear subspace over $\mathbf{Q}$, and the latter is easily verified algorithmically. Notice, that a more economical algorithm can be constructed by analyzing the proof of the classification theorem for finitely generated abelian groups. By Proposition 1.4.4 [49], one can effectively construct a basis $\bar{c}^{(1)}, \bar{c}^{(2)}$ of the free (non-abelian) group $\pi_{1}\left(\Gamma, V_{0}\right)$ so that $\widetilde{\bar{c}}^{(1)}, \widetilde{\bar{c}}^{(2)}$ are the natural images of the elements $\bar{c}^{(1)}, \bar{c}^{(2)}$ in $\widetilde{Z}$.

Now assume that $\langle\mathcal{P}, R\rangle$ is an arbitrary periodic structure of a generalized equation $\Omega$, not necessarily connected. Let $\Gamma_{1}, \ldots, \Gamma_{r}$ be the connected components of the graph $\Gamma$. The labels of edges of the component $\Gamma_{i}$ form in the equation $\Omega$ a union of closed sections from $\mathcal{P}$; moreover, if a base $\mu \in \mathcal{P}$ belongs to such a section, then its dual $\Delta(\mu)$, by condition $\mathrm{f}$ ) of Definition 6.3 also possesses this property. Therefore, by taking for $\mathcal{P}_{i}$ the set of labels of edges from $\Gamma_{i}$ belonging to $\mathcal{P}$, sections to which these labels belong, and bases $\mu \in \mathcal{P}$ belonging to these sections, and restricting in the corresponding way the relation $R$, we obtain a periodic connected structure $\left\langle\mathcal{P}_{i}, R_{i}\right\rangle$ with the graph $\Gamma_{i}$.

The notation $\left\langle\mathcal{P}^{\prime}, R^{\prime}\right\rangle \subseteq\langle\mathcal{P}, R\rangle$ means that $\mathcal{P}^{\prime} \subseteq \mathcal{P}$, and the relation $R^{\prime}$ is a restriction of the relation $R$. In particular, $\left\langle\mathcal{P}_{i}, R_{i}\right\rangle \subseteq\langle\mathcal{P}, R\rangle$ in the situation described in the previous paragraph. It suffices to restrict ourselves to the case of a connected periodic structure.

Let $e_{1}, \ldots, e_{m}$ be all the edges of the graph $\Gamma$ from $T \backslash T_{0}$. Since $T_{0}$ is the spanning forest of the graph $\Gamma_{0}$, it follows that $h\left(e_{1}\right), \ldots, h\left(e_{m}\right) \in \mathcal{P}$. Let $F(\Omega)$ be a free group generated by the variables of $\Omega$. Consider in the group $F(\Omega)$ a new basis $A, \bar{x}$ consisting of $A$, variables not belonging to the closed sections from $\mathcal{P}$, variables $\{h(e) \mid e \in T\}$ and words $h\left(\bar{c}^{(1)}\right), h\left(\bar{c}^{(2)}\right)$. We are to study in more detail how the unknowns $h\left(e_{i}\right)(1 \leq i \leq m)$ can participate in the equations from $\Omega^{*}$ rewritten in this basis.

If $h_{k}$ does not lie on a closed section from $\mathcal{P}$, or $h_{k} \notin \mathcal{P}$, but $e \in T$ (where $h(e)=$ $\left.h_{k}\right)$, then $h_{k}$ belongs to the basis $\bar{x}, A$ and is distinct from each of $h\left(e_{1}\right), \ldots, h\left(e_{m}\right)$. Now let $h(e)=h_{k}, h_{k} \notin \mathcal{P}$ and $e \notin T$. Since $e \in \Gamma_{0}$, the vertices $(k)$ and $(k+1)$ lie in the same connected component of the graph $\Gamma_{0}$, and hence they are 
connected by a path $s$ in the forest $T_{0}$. Furthermore, $r_{1}$ and $s r_{2}^{-1}$ are paths in the tree $T$ connecting the vertices $(k)$ and $V_{0}$; consequently, $r_{1}=s r_{2}^{-1}$. Thus, $e=s r_{2}^{-1} c_{e} r_{2}$ and $h_{k}=h(s) h\left(r_{2}\right)^{-1} h\left(c_{e}\right) h\left(r_{2}\right)$. The unknown $h\left(e_{i}\right) \quad(1 \leq i \leq m)$ can occur in the right-hand side of the expression obtained (written in the basis $\bar{x}, A)$ only in $h\left(r_{2}\right)$ and at most once. Moreover, the sign of this occurrence (if it exists) depends only on the orientation of the edge $e_{i}$ with respect to the root $V_{0}$ of the tree $T$. If $r_{2}=r_{2}^{\prime} e_{i}^{ \pm 1} r_{2}^{\prime \prime}$, then all the occurrences of the unknown $h\left(e_{i}\right)$ in the words $h_{k}$ written in the basis $\bar{x}, A$, with $h_{k} \notin \mathcal{P}$, are contained in the occurrences of words of the form $h\left(e_{i}\right)^{\mp 1} h\left(\left(r_{2}^{\prime}\right)^{-1} c_{e} r_{2}^{\prime}\right) h\left(e_{i}\right)^{ \pm 1}$, i.e., in occurrences of the form $h\left(e_{i}\right)^{\mp 1} h(c) h\left(e_{i}\right)^{ \pm 1}$, where $c$ is a certain cycle of the graph $\Gamma$ starting at the initial vertex of the edge $e_{i}^{ \pm 1}$.

Therefore all the occurrences of $h\left(e_{i}\right), i=1, \ldots, m$ in the equations corresponding to $\mu \notin \mathcal{P}$ are of the form $h\left(e_{i}^{-1}\right) h(c) h\left(e_{i}\right)$. Also, $h\left(e_{i}\right)$ does not occur in the equations corresponding to $\mu \in \mathcal{P}$ in the basis $A, \bar{x}$. Let $V_{i}$ be the initial vertex of the edge $e_{i}$. The system $\Omega^{*}$ is equivalent to the following system: we introduce new variables $\bar{u}^{(i)}=\left\{u_{i e} \mid e \notin T, e \notin \mathcal{P}\right\}, \bar{z}^{(i)}=\left\{z_{i e} \mid 1 \leq i \leq m, e \notin T, e \notin \mathcal{P}\right\}$ and add to $\Omega^{*}$ equations

$$
\begin{gathered}
u_{i e}=h\left(r\left(V_{0}, V_{i}\right)^{-1} c_{e} r\left(V_{0}, V_{i}\right)\right), \\
h\left(e_{i}\right)^{-1} u_{i e} h\left(e_{i}\right)=z_{i e}, \\
{\left[u_{i e_{1}}, u_{i e_{2}}\right]=1,}
\end{gathered}
$$

where $e$ runs over the list of edges not belonging to $T$ and $i$ is fixed. Because $h\left(e_{i}\right)$ does not belong to the right part of [26), we can rewrite $\Omega^{*}$ in the form $\bar{\psi}\left(\bar{x}, \bar{z}^{(i)}, A\right)=1$, such that $h\left(e_{i}\right)$ does not occur in $\bar{\psi}$ and equations (27). Include now all the variables except $h\left(e_{i}\right)$ into the list $\bar{z}$. Let $\bar{\theta}$ consist of (27). Then $F_{R(\Omega)}$ is isomorphic to $F_{\bar{\theta} \& R(\bar{\psi})}$, and it is a HNN extension of the group $F_{R(\psi)}$ with abelian associated subgroups generated by $\bar{u}^{(i)}$ and $\bar{z}^{(i)}$ and a stable letter $h\left(e_{i}\right)$.

Consider now the graph obtained from $\Gamma$ by deleting edges from $\mathcal{P} \backslash T$. Denote it $\bar{\Gamma}$. Every variable $h(e)=h_{k}$, where $h_{k} \notin \mathcal{P}$ and $\bar{e} \notin T$, can be expressed in terms of $h\left(c_{\bar{e}}\right)$, variables $h(e)$ for $e \in T_{0}$ and variables $\bar{z}^{(i)}, i=1, \ldots m$ which correspond to cycles in $\bar{\Gamma}$ with initial vertices $V_{i}$ for each $e_{i}$. Therefore we can rewrite $\Omega^{*}$ in the following form: equations (27) for all $i=1, \ldots, m$ which we denote by $\theta$ and equations $\psi\left(\bar{x}, \bar{z}^{(1)}, \ldots, \bar{z}^{(m)}, A\right)$ such that $h\left(e_{i}\right), i=1, \ldots, m$ do not occur in $\psi$. Then $F_{R(\Omega)}$ is isomorphic to $F_{\theta \& R(\psi)}$, and is a fundamental group of a graph of groups that has one vertex with the vertex group generated by variables $\bar{x}, \bar{z}^{(1)}, \ldots, \bar{z}^{(m)}, A$ except $h\left(e_{1}\right), \ldots, h\left(e_{m}\right)$ and $m$ edges with the corresponding stable letters $h\left(e_{1}\right), \ldots, h\left(e_{m}\right)$.

If a solution $\bar{H}$ of $\Omega$ is periodic with a period $P$, and $\mathcal{P}(H, P)=<\mathcal{P}, R>$, then in a fully residually free quotient $F_{\omega R(\Omega)}$ the groups generated by the images of $\bar{u}^{(i)}$ and $\bar{z}^{(i)}$ are cyclic, because all the cycles in $\bar{u}^{(i)}$ have bounded (in terms of $P$ ) length.

Notice, that without loss of generality we can assume that $v_{0}$ corresponds to the beginning of the period $P$. 
LEMMA 6.7. Let $\Omega$ be a consistent generalized equation periodized with respect to a periodic structure $\langle\mathcal{P}, R\rangle$ with empty set $N(\mathcal{P})$. Then the following is true.

(1) Let $K$ be the subgroup of $F_{R(\Omega)}$ generated by variables $h_{k}$ not belonging to closed sections from $\mathcal{P}, h(e), e \in T_{0}, h\left(\bar{c}^{(1)}\right)$ and $\bar{z}^{(i)}, i=1, \ldots, m$. If $\left|\bar{c}^{(2)}\right|=s \geq 1$, then the group $F_{R(\Omega)}$ splits as a fundamental group of a graph of groups with two vertices, where one vertex group is $K$ and the other is a free abelian group generated by $\bar{c}^{(2)}$ and $\bar{c}^{(1)}$. The edge group is generated by $\bar{c}^{(1)}$. The other edges are loops at the vertex with vertex group $K$ and have stable letters $h\left(e_{i}\right), i=1, \ldots, m$.

If $\Omega$ consists of several periodic structures, then in each fully residually free quotient $F_{\omega R(\Omega)}$ at least for one of them the image of $\left\langle\bar{c}^{(1)}\right\rangle$ is a cyclic subgroup. If $\bar{c}^{(2)}=\emptyset$, then there is no vertex with abelian vertex group.

(2) If $e \in \mathcal{P} \backslash T, c$ and $c_{e}$ have the same initial vertex, then the mapping defined by $h\left(c_{e}\right) \rightarrow h(c)^{k} h\left(c_{e}\right)$ and fixing all the other generators can be extended to an automorphism of $F_{R(\Omega)}$.

(3) Let $A, \bar{x}$ be the generators of the group $F_{R(\Omega)}$ constructed above. If $e_{i} \in$ $\mathcal{P} \cap T$, and $c$ is a cycle beginning at the initial vertex of $e_{i}$, then the mapping defined as $h\left(e_{i}\right) \rightarrow h(c)^{k} h\left(e_{i}\right)$ ( $k$ is any integer) on the generator $h\left(e_{i}\right)$ and fixing all the other generators can be extended to an automorphism of $F_{R(\Omega)}$.

(4) Let $H$ be a solution of $\Omega$ periodic with respect to a period $P$ and $\mathcal{P}(H, P)=$ $\langle\mathcal{P}, R\rangle$. If the solution $H$ of $\Omega$ cannot be obtained by a substitution from a shorter solution then there is a cycle $c$ in $\tilde{Z}$ such that $H(c)=P^{n_{0}}$, and $n_{0}<2 \rho$.

(5) One can choose the basis $\bar{c}^{(1)}$ so that for any $c \in \bar{c}^{(1)}, H(c)=P^{n}$, where $n<2 \rho$. If the group $\left\langle\bar{c}^{(1)}\right\rangle$ is not cyclic, then there exists a finite number of non-trivial cycles $c_{1}, \ldots, c_{k}$ in the graph $\Gamma_{0}$ such that for any solution $H$ there is an equality $H\left(c_{i}\right)=1$ for some $i=1, \ldots, k$.

Proof. To prove the first statement we add to the system $\Omega^{*}$ equations from Definition [6.6] for all pairs of cycles $c_{e_{1}}, c_{e_{2}} \quad\left(e_{1}, e_{2} \notin T\right)$. We have an equivalent system. Let $y_{1}=h\left(c_{1}^{(2)}\right), \ldots, y_{s}=h\left(c_{s}^{(2)}\right)$ with the rest of the variables from the list $\bar{x}$ considered as variables from $\bar{t}$. All the equations of the system $\Omega^{*}$ can be rewritten modulo commutativity relations from Definition 6.6 in the variables $\bar{t}$ as a system $\gamma(\bar{t}, A)=1$. The commutativity relations can be rewritten in the form

$$
\left[h(c), h\left(c^{\prime}\right)\right]=\left[y_{i}, y_{j}\right]=\left[y_{i}, h(c)\right]=1,\left(c, c^{\prime} \in \bar{c}^{(1)}, i, j=1, \ldots, s\right) .
$$

The system $\phi$, obtained as a union of equations (29) and equations from $\gamma$ is equivalent to $\Omega^{*}$ so there is a natural isomorphism between $F_{R(\Omega)}$ and $F_{R(\phi)}$. The first statement now follows from the isomorphism between $F_{R(\Omega)}$ and $F_{\theta \& R \psi}$ obtained before the formulation of the lemma.

Statements (2) and (3) are obvious now. Indeed, the mapping sending each element $y_{i}$ to $h(c) y_{i}$ for some cycle $c$ and identical on the generators from $A, \bar{t}$ can be extended to an automorphism of $F_{R(\Omega)}$.

We will now prove statement (4). Let $H$ be a solution of the generalized equation $\Omega$ periodic with respect to some period $P,\langle\mathcal{P}, R\rangle$ a connected component of the structure $\mathcal{P}(H, P)$, and assume the solution $H$ cannot be obtained from a shorter solution by a substitution. Without loss of generality, we can assume that 
$\delta\left(V_{0}\right)=\Lambda P$. Then, by Lemma 6.5 there is a homomorphism $\gamma: \widetilde{Z} \rightarrow \mathbb{Z}$ such that for every cycle $c \in \pi_{1}\left(\Gamma, V_{0}\right)$ the condition $H(c)=P^{\gamma(\tilde{c})}$ holds. Let us first verify that if for some variable $h_{k} \in \mathcal{P}$

$$
d\left(H_{k}\right) \geq 2 \rho^{2} d(P),
$$

then $\gamma(\tilde{\mathbb{Z}})$ contains a certain $n$ such that $1 \leq n \leq 2 \rho$, where $\rho$ is the number of unknowns in the equation $\Omega$. To verify this, let us construct a chain

$$
(\Omega, H)=\left(\Omega_{0}, H^{(0)}\right) \rightarrow\left(\Omega_{1}, H^{(1)}\right) \rightarrow \cdots \rightarrow\left(\Omega_{t}, H^{(t)}\right),
$$

in which every term is obtained from the previous one by extending a certain boundary through a certain base $\mu \in \mathcal{P}$ with the help of the E.5 transformation. The construction of the chain (31) terminates when all boundaries intersecting bases from $\mathcal{P}$ turn out to be extended through these bases. Let $\Omega_{i}^{\prime}$ be the equation obtained from $\Omega_{i}$ by deleting all boundary connections. It is obvious that the solution $H^{(i)}$ of the equation $\Omega_{i}^{\prime}$ is periodic with respect to the period $P$. Denote by $\left\langle\mathcal{P}_{i}, R_{i}\right\rangle$ the periodic structure $\mathcal{P}\left(H^{(i)}, P\right)$ of the equation $\Omega_{i}^{\prime}$ restricted to the closed sections of $\mathcal{P}$, and by $\Gamma^{(i)}, \widetilde{Z}^{(i)}, \gamma_{i}$ the corresponding graph, abelian group of cycles and homomorphism $\widetilde{Z}^{(i)} \rightarrow \mathbb{Z}$, respectively.

If $\langle P, \mu, q\rangle(\mu \in \mathcal{P})$ is a boundary connection of the equation $\Omega_{i}(1 \leq i \leq t)$, then $\delta(p)=\delta(q)$; therefore all the graphs $\Gamma^{(0)}, \Gamma^{(1)}, \ldots, \Gamma^{(t)}$ have the same set of vertices, whose cardinality does not exceed $\rho$. The solution $H^{(t)}$ of the equation $\Omega_{t}$ is minimal with respect to the trivial group of automorphisms. Suppose that for some unknown $h_{l}$ lying on a closed section from $\mathcal{P}$ the inequality $d\left(H_{l}^{(t)}\right)>2 d(P)$ holds. In the vector $H^{(t)}$, replace all the components that are graphically equal to $\left(H_{l}^{(t)}\right)^{ \pm 1}$ and correspond to the unknowns lying on the closed sections from $\mathcal{P}$, by a letter $u^{ \pm 1}$ of the alphabet $\Sigma_{2}$ not participating in the solution $H^{(t)}$. The resulting vector obviously satisfies the conditions of non emptiness and irreducibility. It satisfies all basic equations of the generalized equation $\Omega_{t}$ with numbers $\mu \in \mathcal{P}$ and all the corresponding boundary equations, since in the equation $\Omega_{t}$ all the boundaries from $\mathcal{P}$ are extended through all possible boundaries. If, on the other hand, $\mu \notin \mathcal{P}$, then for every unknown $h_{k} \in \mu$ of the equation $\Omega$ lying on a closed section from $\mathcal{P}$, we have $h_{k} \notin \mathcal{P}$ and, consequently, $d\left(H_{k}\right) \leq 2 d(P)$. In particular, this inequality holds for the unknowns $h_{k} \in \mu$ of the equation $\Omega_{t}$; therefore, such unknowns have not been replaced in the vector $H^{(t)}$. Consequently, the vector constructed is a solution to the equation $\Omega_{t}$, which contradicts the minimality of the solution $H^{(t)}$.

Thus we have established the fact that $d\left(H_{l}^{(t)}\right) \leq 2 d(P)$, if $h_{l}$ lies on a closed section from $\mathcal{P}$. In particular, the unknown $h_{k}$ of the equation $\Omega$ for which inequality (30) holds was divided during the transition to the equation $\Omega_{t}$ into at least $\rho$ distinct unknowns. Since the graph $\Gamma^{(t)}$ contains at most $\rho$ vertices, in the equation $\Omega_{t}^{\prime}$ we can choose boundaries $l$ and $l^{\prime}$ such that $l<l^{\prime},(l)=\left(l^{\prime}\right)$, and $l^{\prime}-l \leq \rho$. The word $h\left[l, l^{\prime}\right]$ is a label of a cycle $c_{t}$ of the graph $\Gamma^{(t)}$ for which $0<d\left(H\left(c_{t}\right)\right) \leq$ $2 \rho d(P)$, i.e., $\gamma_{t}\left(\widetilde{Z}^{(t)}\right)$ contains a number $n$ with the property $1 \leq n \leq 2 \rho$. By $\pi_{i j}$ $(0 \leq i<j \leq t)$ we denote from now on the homomorphism $G\left(\Omega_{i}^{*}\right) \rightarrow G\left(\Omega_{j}^{*}\right)$ defined by the sequence (31). It remains to prove the existence of a cycle $c_{0}$ of the graph $\Gamma$ for which $\pi_{0 t}\left(h\left(c_{0}\right)\right)=h\left(c_{t}\right)$. 
To do this, it suffices to show that for every path $r_{i+1}: V \rightarrow V^{\prime}$ in the graph $\Gamma^{(i+1)}$ there exists a path $r_{i}: V \rightarrow V^{\prime}$ in the graph $\Gamma^{(i)}$ such that $\pi_{i, i+1}\left(h\left(r_{i}\right)\right)=$ $h\left(r_{i+1}\right)$. In turn, it suffices to verify the latter statement for the case where $r_{i+1}$ is the edge $e$. If the unknown $h(e)$ of the equation $\Omega_{i+1}$ is also an unknown of the equation $\Omega_{i}$, then this is obvious. Otherwise one should use the formulas

$$
\begin{gathered}
\pi_{i, i+1}^{-1}\left(h^{\prime}\right)=h[\alpha(\Delta(\mu)), q]^{-1} h[\alpha(\mu), p], \\
\pi_{i, i+1}^{-1}\left(h^{\prime \prime}\right)=h[\alpha(\mu), p]^{-1} h[\alpha(\Delta(\mu)), q+1],
\end{gathered}
$$

defining the inverse isomorphism to $\pi_{i, i+1}$, and notice that the right-hand sides of these formulas are labels of paths in $\Gamma^{(i)}$, since $(\alpha(\mu))=(\alpha(\Delta(\mu)))$.

Thus, we have deduced from (30) that $\gamma(\widetilde{Z})$ is a nonzero subgroup in $\mathbb{Z}$ whose generator $n_{0}$ satisfies the inequality $\left|n_{0}\right| \leq 2 \rho$.

To prove assertion (5) we have to show that each simple cycle in the graph $\Gamma_{0}$ has length less than $2 \rho$. This is obvious, because the total number of edges in $\Gamma_{0}$ is not more than $\rho$ and corresponding variables do not belong to $\mathcal{P}$. The image of the group $\left\langle\bar{c}^{(1)}\right\rangle$ in $F$ is cyclic, therefore one of the finite number of equalities between cycles must hold for any solution.

We now introduce the notion of a canonical group of automorphisms corresponding to a connected periodic structure.

DEFINITION 6.8. In the case when the family of bases $N \mathcal{P}$ is empty automorphisms described in Lemma 6.7 for $e_{1}, \ldots, e_{m} \in T \backslash T_{0}$ and all $c_{e}$ for $e \in \mathcal{P} \backslash T$ generate the canonical group of automorphisms $P_{0}$ corresponding to a connected periodic structure.

LEMma 6.9. Let $\Omega$ be a nondegenerate generalized equation with no boundary connections, periodized with respect to the periodic structure $\langle\mathcal{P}, R\rangle$. Suppose that the set $N \mathcal{P}$ is empty. Let $H$ be a solution of $\Omega$ periodic with respect to a period $P$ and $\mathcal{P}(H, P)=<\mathcal{P}, R>$. Combining canonical automorphisms of $F_{R(\Omega)}$ one can get a solution $H^{+}$of $\Omega$ with the property that for any $h_{k} \in \mathcal{P}$ such that $H_{k}=P_{2} P^{n_{k}} P_{1}$ $\left(P_{2}\right.$ and $P_{1}$ are an end and a beginning of $\left.P\right), H_{k}^{+}=P_{2} P^{n_{k}^{+}} P_{1}$, where $n_{k}, n_{k}^{+}>0$ and the numbers $n_{k}^{+}$'s are bounded by a certain computable function $f_{2}(\Omega, \mathcal{P}, R)$. For all $h_{k} \notin \mathcal{P} H_{k}=H_{k}^{+}$.

Proof. Let $\delta((k))=P_{1}^{(k)} P_{2}^{(k)}$. Denote by $t\left(\mu, h_{k}\right)$ the number of occurrences of the edge with label $h_{k}$ in the cycle $c_{\mu}$, calculated taking into account the orientation. Let

$$
H_{k}=P_{2}^{(k)} P^{n_{k}} P_{1}^{(k+1)}
$$

( $h_{k}$ lies on a closed section from $\mathcal{P}$ ), where the equality in (32) is graphic whenever $h_{k} \in \mathcal{P}$. Direct calculations show that

$$
H\left(b_{\mu}\right)=P^{\sum_{k} t\left(\mu, h_{k}\right)\left(n_{k}+1\right)} .
$$


This equation implies that the vector $\left\{n_{k}\right\}$ is a solution to the following system of Diophantine equations in variables $\left\{z_{k} \mid h_{k} \in \mathcal{P}\right\}$ :

$$
\sum_{h_{k} \in \mathcal{P}} t\left(\mu, h_{k}\right) z_{k}+\sum_{h_{k} \notin \mathcal{P}} t\left(\mu, h_{k}\right) n_{k}=0,
$$

$\mu \in \mathcal{P}$. Note that the number of unknowns is bounded, and coefficients of this system are bounded from above $\left(\left|n_{k}\right| \leq 2\right.$ for $\left.h_{k} \notin \mathcal{P}\right)$ by a certain computable function of $\Omega, \mathcal{P}$, and $R$. Obviously, $\left(P_{2}^{(k)}\right)^{-1} H_{k}^{+} H_{k}^{-1} P_{2}^{(k)}=P^{n_{k}^{+}-n_{k}}$ commutes with $H(c)$, where $c$ is a cycle such that $H(c)=P^{n_{0}}, n_{0}<2 \rho$.

If system (34) has only one solution, then it is bounded. Suppose it has infinitely many solutions. Then $\left(z_{1}, \ldots, z_{k}, \ldots\right)$ is a composition of a bounded solution of (34) and a linear combination of independent solutions of the corresponding homogeneous system. Applying canonical automorphisms from Lemma 6.7 we can decrease the coefficients of this linear combination to obtain a bounded solution $H^{+}$. Hence for $h_{k}=h\left(e_{i}\right), e_{i} \in \mathcal{P}$, the value $H_{k}$ can be obtained by a composition of a canonical automorphism (Lemma 6.7) and a suitable bounded solution $\mathrm{H}^{+}$of $\Omega$.

6.3. Case 2. Set $N \mathcal{P}$ is non-empty. Construct an oriented graph $B \Gamma$ with the same set of vertices as $\Gamma$. For each item $h_{k} \notin \mathcal{P}$ such that $h_{k}$ lie on a certain closed section from $\mathcal{P}$ introduce an edge $e$ leading from $(k)$ to $(k+1)$ and $e^{-1}$ leading from $(k+1)$ to $(k)$. For each pair of bases $\mu, \Delta(\mu) \in \mathcal{P}$ introduce an edge $e$ leading from $(\alpha(\mu))=(\alpha(\Delta(\mu)))$ to $(\beta(\mu))=(\beta(\delta(\mu)))$ and $e^{-1}$ leading from $(\beta(\mu))$ to $(\alpha(\mu))$. For each base $\mu \in N \mathcal{P}$ introduce an edge $e$ leading from $(\alpha(\mu)$ to $(\beta(\mu))$ and $e^{-1}$ leading from $(\beta(\mu))$ to $(\alpha(\mu))$. denote by $B \Gamma_{0}$ the subgraph with the same set of vertices and edges corresponding to bases not from $\mathcal{P}$. Choose a maximal subforest $B T_{0}$ in the graph $B \Gamma_{0}$ and extend it to a maximal subforest $B T$ of the graph $B \Gamma$. Since $\mathcal{P}$ is connected, $B T$ is a tree.

Lemma 6.10. Let $H$ be a solution of a generalized equation $\Omega$ periodic with respect to a period $P,\langle\mathcal{P}, R\rangle=\mathcal{P}(H, P)$; c be a cycle in the graph $B \Gamma$ at the vertex $(l) ; \delta(l)=P_{1} P_{2}$. Then there exists $n \in \mathbb{Z}$ such that $H(c)=\left(P_{2} P_{1}\right)^{n}$.

As we did in the graph $\Gamma$, we choose a vertex $V_{0}$. Let $r\left(V_{0}, V\right)$ be the unique path in $B T$ from $V_{0}$ to $V$. For every edge $e=e(\mu): V \rightarrow V^{\prime}$ not lying in $B T$, introduce a cycle $c_{\mu}=r\left(V_{0}, V\right) e(\mu) r\left(V_{0}, V^{\prime}\right)^{-1}$. For every edge $e=e\left(h_{k}\right): V \rightarrow V^{\prime}$ not lying in $B T$, introduce a cycle $c_{h_{k}}=r\left(V_{0}, V\right) e\left(h_{k}\right) r\left(V_{0}, V^{\prime}\right)^{-1}$.

It suffices to restrict ourselves to the case of a connected periodic structure. If $e=e\left(h_{k}\right)$, we denote $h(e)=h_{k}$; if $e=e(\mu)$, then $h(e)=\mu$. Let $e_{1}, \ldots, e_{m}$ be all the edges of the graph $B \Gamma$ from $B T \backslash B T_{0}$. Since $B T_{0}$ is the spanning forest of the graph $B \Gamma_{0}$, it follows that $h\left(e_{1}\right), \ldots, h\left(e_{m}\right) \in \mathcal{P}$. Consider in the free group $F(\Omega)$ a new basis $A, \bar{x}$ consisting of $A$, items $h_{k}$ such that $h_{k}$ does not belong to closed sections from $\mathcal{P}$, variables $\{h(e) \mid e \in T\}$ and words from $h\left(C^{(1)}\right), h\left(C^{(2)}\right)$, where the set $C^{(1)}, C^{(2)}$ form a basis of the free group $\pi\left(B \Gamma, V_{0}\right), C^{(1)}$ correspond to the cycles that represent the identity in $F_{R(\Omega)}$ (if $V$ and $V^{\prime}$ are initial and terminal vertices of some closed section in $\mathcal{P}$ and $r$ and $r_{1}$ are different paths from $V$ to $V^{\prime}$, then $r\left(V_{0}, V\right) r r_{1}^{-1} r\left(V_{0}, V\right)^{-1}$ represents the identity), cycles $c_{\mu}, \mu \in N \mathcal{P}$ and $c_{h_{k}}$, $h_{k} \notin \mathcal{P}$; and $C^{(2)}$ contains the rest of the basis of $\pi\left(B \Gamma, V_{0}\right)$. 
We study in more detail how the unknowns $h\left(e_{i}\right)(1 \leq i \leq m)$ can participate in the equations from $\Omega^{*}$ rewritten in this basis.

If $h_{k}$ does not lie on a closed section from $\mathcal{P}$, or $h_{k}=h(e), h(\mu)=h(e) \notin \mathcal{P}$, but $e \in T$, then $h(\mu)$ or $h_{k}$ belongs to the basis $\bar{x}, \bar{a}$ and is distinct from each of $h\left(e_{1}\right), \ldots, h\left(e_{m}\right)$. Now let $h(e)=h(\mu), h(\mu) \notin \mathcal{P}$ and $e \notin T$. Since $e \in B \Gamma_{0}$, the vertices $(\alpha(\mu))$ and $(\beta(\mu))$ lie in the same connected component of the graph $B \Gamma_{0}$, and hence are connected by a path $s$ in the forest $B T_{0}$. Furthermore, $r_{1}$ and $s r_{2}^{-1}$ are paths in the tree $B T$ connecting the vertices $(\alpha(\mu))$ and $V_{0}$; consequently, $r_{1}=s r_{2}^{-1}$. Thus, $e=s r_{2}^{-1} c_{e} r_{2}$ and $h(\mu)=h(s) h\left(r_{2}\right)^{-1} h\left(c_{e}\right) h\left(r_{2}\right)$. The unknown $h\left(e_{i}\right)(1 \leq i \leq m)$ can occur in the right-hand side of the expression obtained (written in the basis $\bar{x}, A$ ) only in $h\left(r_{2}\right)$ and at most once. Moreover, the sign of this occurrence (if it exists) depends only on the orientation of the edge $e_{i}$ with respect to the root $V_{0}$ of the tree $T$. If $r_{2}=r_{2}^{\prime} e_{i}^{ \pm 1} r_{2}^{\prime \prime}$, then all the occurrences of the unknown $h\left(e_{i}\right)$ in the words $h(\mu)$ written in the basis $\bar{x}, A$, with $h(\mu) \notin \mathcal{P}$, are contained in the occurrences of words of the form $h\left(e_{i}\right)^{\mp 1} h\left(\left(r_{2}^{\prime}\right)^{-1} c_{e} r_{2}^{\prime}\right) h\left(e_{i}\right)^{ \pm 1}$, i.e., in occurrences of the form $h\left(e_{i}\right)^{\mp 1} h(c) h\left(e_{i}\right)^{ \pm 1}$, where $c$ is a certain cycle of the graph $B \Gamma$ starting at the initial vertex of the edge $e_{i}^{ \pm 1}$. Similarly, all the occurences of the unknown $h\left(e_{i}\right)$ in the words $h_{k}$ written in the basis $\bar{x}, A$, with $h_{k} \notin \mathcal{P}$, are contained in occurrences of words of the form $h\left(e_{i}\right)^{\mp 1} h(c) h\left(e_{i}\right)^{ \pm 1}$.

Therefore all the occurences of $h\left(e_{i}\right), i=1, \ldots, m$ in the equations corresponding to $\mu \notin \mathcal{P}$ are of the form $h\left(e_{i}^{-1}\right) h(c) h\left(e_{i}\right)$. Also, cycles from $C^{(1)}$ that represent the identity and not in $B \Gamma_{0}$ are basis elements themselves. This implies

LEMma 6.11. 1 . Let $K$ be the subgroup of $F_{R(\Omega)}$ generated by variables $h_{k}$ not belonging to closed sections from $\mathcal{P}, h(e), e \in B T_{0}, h\left(C^{(1)}\right)$ and $\bar{z}^{(i)}, i=1, \ldots, m$, where elements $\bar{z}^{(i)}$ are defined similarly to the case of empty $\mathcal{N} P$.

(1) If $\left|C^{(2)}\right|=s \geq 1$, then the group $F_{R(\Omega)}$ splits as a fundamental group of a graph of groups with two vertices, where one vertex group is $K$ and the other is a free abelian group generated by $C^{(2)}$ and $C^{(1)}$. The edge group is generated by $C^{(1)}$. The other edges are loops at the vertex with vertex group $K$ and have stable letters $h(e), e \in B T \backslash B T_{0}$. If $C^{(2)}=\emptyset$, then there is no vertex with abelian vertex group.

(2) Let $H$ be a solution of $\Omega$ periodic with respect to a period $P$ and $\langle\mathcal{P}, R\rangle=$ $\mathcal{P}(H, P)$. Let $P_{1} P_{2}$ be a partition of $P$ corresponding to the initial vertex of $e_{i}$. A transformation $H\left(e_{i}\right) \rightarrow P_{2} P_{1} H\left(e_{i}\right), i \in\{1, \ldots, m\}$, which is identical on all the other elements from $A, H(\bar{x})$, gives another solution of $\Omega^{*}$. If $c$ is a cycle beginning at the initial vertex of $e_{i}$, then the transformation $h\left(e_{i}\right) \rightarrow h(c) h\left(e_{i}\right)$ which is identical on all other elements from $A, \bar{x}$, is an automorphism of $F_{R(\Omega)}$.

(3) If $c(e) \in C^{(2)}$, then the transformation $H(c(e)) \rightarrow P H(c(e))$ which is identical on all other elements from $A, H(\bar{x})$, gives another solution of $\Omega^{*}$. A transformation $h(c(e)) \rightarrow h(c) h(c(e))$ which is identical on all other elements from $A, \bar{x}$, is an automorphism of $F_{R(\Omega)}$.

DEFINITION 6.12. If $\Omega$ is a nondegenerate generalized equation periodic with respect to a connected periodic structure $\langle\mathcal{P}, R\rangle$ and the set $N \mathcal{P}$ is non-empty, we consider the group $\bar{A}(\Omega)$ of transformations of solutions of $\Omega^{*}$, where $\bar{A}(\Omega)$ is generated by the transformations defined in Lemma 6.11] If these transformations are automorphisms, the group will be denoted $A(\Omega)$. 
DEFINITION 6.13. In the case when for a connected periodic structure $\langle\mathcal{P}, R\rangle$, the set $C^{(2)}$ has more than one element or $C^{(2)}$ has one element, and $C^{(1)}$ contains a cycle formed by variables $h_{k}$ not from $\mathcal{P}$, the periodic structure will be called singular.

This definition coincide with the definition of singular periodic structure given in [36]) in the case of empty set $\Lambda$.

Lemma 6.11 implies the following

LEMMA 6.14. Let $\Omega$ be a nondegenerate generalized equation with no boundary connections, periodized with respect to a singular periodic structure $\langle\mathcal{P}, R\rangle$. Let $H$ be a solution of $\Omega$ periodic with respect to a period $P$ and $\langle\mathcal{P}, R\rangle=\mathcal{P}(H, P)$. Combining canonical automorphisms from $A(\Omega)$ one can get a solution $H^{+}$of $\Omega^{*}$ with the following properties:

(1) For any $h_{k} \in \mathcal{P}$ such that $H_{k}=P_{2} P^{n_{k}} P_{1}\left(P_{2}\right.$ and $P_{1}$ are an end and a beginning of $P$ ) $H_{k}^{+}=P_{2} P^{n_{k}^{+}} P_{1}$, where $n_{k}, n_{k}^{+} \in \mathbb{Z}$;

(2) For any $h_{k} \notin \mathcal{P}, H_{k}=H_{k}^{+}$;

(3) For any base $\mu \notin \mathcal{P}, H(\mu)=H^{+}(\mu)$;

(4) There exists a nontrivial cycle $c$ such that $H^{+}(c)=1$.

Notice, that in the case described in the lemma, solution $H^{+}$satisfies a proper equation. Solution $H^{+}$is not necessarily a solution of the generalized equation $\Omega$, but we will modify $\Omega$ into a generalized equation $\Omega(\mathcal{P}, B T)$. This modification will be called the first minimal replacement. Equation $\Omega(\mathcal{P}, B T)$ will have the following properties:

1) $\Omega(\mathcal{P}, B T)$ contains all the same parameter sections and closed sections which are not in $\mathcal{P}$, as $\Omega$;

2) $H^{+}$is a solution of $\Omega(\mathcal{P}, B T)$;

3) group $F_{R(\Omega(\mathcal{P}, B T))}$ is generated by the same set of variables $h_{1}, \ldots, h_{\delta}$;

4) the mapping $h_{i} \rightarrow h_{i}$ is a proper homomorphism from $F_{R(\Omega)}$ onto $F_{R(\Omega(\mathcal{P}, B T))}$;

5) $\Omega(\mathcal{P}, B T)$ has the same set of bases as $\Omega$ and possibly some new bases, but each new base is a product of bases from $\Omega$.

To obtain $\Omega(\mathcal{P}, B T)$ we have to modify the closed sections from $\mathcal{P}$.

The label of each cycle in $B \Gamma$ is a product of some bases $\mu_{1} \ldots \mu_{k}$. Write a generalized equation $\tilde{\Omega}$ for the equations that say that $\mu_{1} \ldots \mu_{k}=1$ for each cycle from $C^{(1)}$ representing the trivial element and for each cycle from $C^{(2)}$. Each $\mu_{i}$ is a product $\mu_{i}=h_{i 1} \ldots h_{i t}$. Due to the first statement of Lemma 6.14 in each product $H_{i j}^{+} H_{i, j+1}^{+}$either there is no cancellations between $H_{i j}^{+}$and $H_{i, j+1}^{+}$, or one of them is completely cancelled in the other. Therefore the same can be said about each pair $H^{+}\left(\mu_{i}\right) H^{+}\left(\mu_{i+1}\right)$, and we can make a cancellation table without cutting items or bases of $\Omega$.

Let $\hat{\Omega}$ be a generalized equation obtained from $\Omega$ by deleting bases from $\mathcal{P} \cup N \mathcal{P}$ and items from $\mathcal{P}$ from the closed sections from $\mathcal{P}$. Take a union of $\tilde{\Omega}$ and $\hat{\Omega}$ on the disjoint set of variables, and add basic equations identifying in $\hat{\Omega}$ and $\tilde{\Omega}$ the same bases that don't belong to $\mathcal{P}$. This gives us $\Omega(\mathcal{P}, B T)$.

Suppose that $C^{(2)}$ for the equation $\Omega$ is either empty or contains one cycle. Suppose also that for each closed section from $\mathcal{P}$ in $\Omega$ there exists a base $\mu$ such that the initial boundary of this section is $\alpha(\mu)$ and the terminal boundary is $\beta(\Delta(\mu))$. 
LEMma 6.15. Suppose that the generalized equation $\Omega$ is periodized with respect to a non-singular periodic structure $\mathcal{P}$. Then for any periodic solution $H$ of $\Omega$ we can choose a tree BT, some set of variables $S=\left\{h_{j_{1}}, \ldots, h_{j_{s}}\right\}$ and a solution $H^{+}$of $\Omega$ equivalent to $H$ with respect to the group of canonical transformations $\bar{A}(\Omega)$ in such a way that each of the bases $\lambda_{i} \in B T \backslash B T_{0}$ can be represented as $\lambda_{i}=\lambda_{i 1} h_{k_{i}} \lambda_{i 2}$, where $h_{k_{i}} \in S$ and for any $h_{j} \in S, d\left(H_{j}^{+}\right)<f_{3} d(P)$, where $f_{3}$ is some constructible function depending on $\Omega$. This representation gives a new generalized equation $\Omega^{\prime}$ periodic with respect to a periodic structure $\mathcal{P}^{\prime}$ with the same period $P$ and all $h_{j} \in S$ considered as variables not from $\mathcal{P}^{\prime}$. The graph $B \Gamma^{\prime}$ for the periodic structure $\mathcal{P}^{\prime}$ has the same set of vertices as $B \Gamma$, has empty set $C^{(2)}$ and $B T^{\prime}=B T_{0}^{\prime}$.

Let $c$ be a cycle from $C^{(1)}$ of minimal length, $d(c)=n_{c} d(P)$. Using canonical automorphisms from $A(\Omega)$ one can transform any solution $H$ of $\Omega$ into a solution $H^{+}$such that for any $h_{j} \in S, d\left(H_{j}^{+}\right) \leq f_{3} d(c)$. Let $\mathcal{P}^{\prime}$ be a periodic structure, in which all $h_{i} \in S$ are considered as variables not from $\mathcal{P}^{\prime}$, then $B \Gamma^{\prime}$ has empty set $C^{(2)}$ and $B T^{\prime}=B T_{0}^{\prime}$.

Proof. Suppose first that $C^{(2)}$ is empty. We prove the statement of the lemma by induction on the number of edges in $B T \backslash B T_{0}$. It is true, when this set is empty. Consider temporarily all the edges in $B T \backslash B T_{0}$ except one edge $e(\lambda)$ as edges corresponding to bases from $N \mathcal{P}$. Then the difference between $B T_{0}$ and $B T$ is one edge.

Changing $\lambda$ by a transformation from $\bar{A}(\Omega)$ we can change only the edges from $B \Gamma$ that could be included into $B T \backslash B T_{0}$ instead of $e$. For each base $\mu \in N \mathcal{P}$, $H(\mu)=P_{2}(\mu) P^{n(\mu)} P_{1}(\mu)$, for each base $\mu \in \mathcal{P}, H(\mu)=P_{2}(\mu) P^{x(\mu)} P_{1}(\mu)$. For each cycle in $C^{(1)}$ representing the identity element we have a linear equation in variables $x(\mu)$ with coefficients depending on $n(\mu)$. We also know that this system has a solution for arbitrary $x(\lambda)$ (where $\lambda \in B T \backslash B T_{0}$ ) and the other $x(\nu)$ are uniquely determined by the value of $x(\lambda)$.

If we write for each variable $h_{k} \in \mathcal{P}, H_{k}=P_{2 k} P^{y_{k}} P_{1 k}$, then the positive unknowns $y_{k}$ 's satisfy the system of equations saying that $H(\mu)=H(\Delta(\mu))$ for bases $\mu \in \mathcal{P}$ and equations saying that $\mu$ is a constant for bases $\mu \in N \mathcal{P}$. Fixing $x(\lambda)$ we automatically fix all the $y_{k}$ 's. Therefore at least one of the $y_{k}$ belonging to $\lambda$ can be taken arbitrary. So there exist some elements $y_{k}$ which can be taken as free variables for the second system of linear equations. Using elementary transformations over $\mathbf{Z}$ we can write the system of equations for $y_{k}$ 's in the form:

$$
\begin{array}{ccccc}
n_{1} y_{1} & 0 & \ldots & =m_{1} y_{k} & +C_{1} \\
& n_{2} y_{2} & \ldots & =m_{2} y_{k} & +C_{2} \\
& & \ddots & & \vdots \\
\vdots & \vdots & & \ddots & \\
& \cdots & n_{k-1} y_{k-1} & =m_{k-1} y_{k} & +C_{k-1},
\end{array}
$$

where $C_{1}, \ldots C_{k}$ are constants depending on parameters, we can suppose that they are sufficiently large positive or negative (small constants we can treat as constants not depending on parameters). Notice that integers $n_{1}, m_{1}, \ldots, n_{k-1}, m_{k-1}$ in this system do not depend on parameters. We can always suppose that all $n_{1}, \ldots, n_{k-1}$ are positive. Notice that $m_{i}$ and $C_{i}$ cannot be simultaneously negative, because in this case it would not be a positive solution of the system. Changing the order of the 
equations we can write first all equation with $m_{i}, C_{i}$ positive, then equations with negative $m_{i}$ and positive $C_{i}$ and, finally, equations with negative $C_{i}$ and positive $m_{i}$. The system will have the form:

$\begin{array}{ccccc}n_{1} y_{1} & 0 & \cdots & =\left|m_{1}\right| y_{k} & +\left|C_{1}\right|, \\ & & \ddots & & \vdots \\ & n_{t} y_{t} & \cdots & =-\left|m_{t}\right| y_{k} & +\left|C_{t}\right|, \\ & & \ddots & & \vdots \\ \vdots & \vdots & & \ddots & \\ & \ldots & n_{s} y_{s} & =\left|m_{s}\right| y_{k} & -\left|C_{s}\right|\end{array}$

If the last block (with negative $C_{s}$ ) is non-empty, we can take a minimal $y_{s}$ of bounded value. Indeed, instead of $y_{s}$ we can always take a remainder of the division of $y_{s}$ by the product $n_{1} \ldots n_{k-1}\left|m_{1} \ldots m_{k-1}\right|$, which is less than this product (or by the product $n_{1} \ldots n_{k-1}\left|m_{1} \ldots m_{k-1}\right| n_{c}$ if we wish to decrease $y_{s}$ by a multiple of $n_{c}$ ). We respectively decrease $y_{k}$ and adjust $y_{i}$ 's in the blocks with positive $C_{i}$ 's. If the third block is not present, we decrease $y_{k}$ taking a remainder of the division of $y_{k}$ by $n_{1} \ldots n_{k-1}$ (or by $n_{1} \ldots n_{k-1} n_{c}$ ) and adjust $y_{i}$ 's. Therefore for some $h_{i}$ belonging to a base which can be included into $B T \backslash B T_{0}, d\left(H^{+}\left(h_{i}\right)\right)<f_{3} d(P)$. Suppose this base is $\lambda$, represent $\lambda=\lambda_{1} h_{i} \lambda_{2}$. Suppose $e(\lambda): V \rightarrow V_{1}$ in $B \Gamma$. Let $V_{2}, V_{3}$ be the vertices in $B \Gamma$ corresponding to the initial and terminal boundary of $h_{k}$. They would be the vertices in $\Gamma$, and $\Gamma$ and $B \Gamma$ have the same set of vertices. To obtain the graph $B \Gamma^{\prime}$ from $B \Gamma$ we have to replace $e(\lambda)$ by three edges $e\left(\lambda_{1}\right): V \rightarrow V_{2}, e\left(h_{k}\right): V_{2} \rightarrow V_{3}$ and $e\left(\lambda_{2}\right): V_{3} \rightarrow V_{1}$. There is no path in $B T_{0}$ from $V_{2}$ to $V_{3}$, because if there were such a path $p$, then we would have the equality $h_{k}=h\left(c_{1}\right) h(p) h\left(c_{2}\right)$, in $F_{R(\Omega)}$, where $c_{1}$ and $c_{2}$ are cycles in $B \Gamma$ beginning in vertices $V_{2}$ and $V_{3}$ respectively. Changing $H_{k}$ we do not change $H\left(c_{1}\right), H\left(c_{2}\right)$ and $H(p)$, because all the cycles are generated by cycles in $C^{(1)}$. Therefore there are paths $r: V \rightarrow V_{2}$ and $r_{1}: V_{3} \rightarrow V_{1}$ in $B T_{0}$, and edges $e\left(\lambda_{1}\right), e\left(\lambda_{2}\right)$ cannot be included in $B T^{\prime} \backslash B T_{0}^{\prime}$ in $B \Gamma^{\prime}$. Therefore $B T^{\prime}=B T_{0}^{\prime}$. Now we can recall that all the edges except one in $B T \backslash B T_{0}$ were temporarily considered as edges in $N \mathcal{P}$. We managed to decrease the number of such edges by one. Induction finishes the proof.

If the set $C^{(2)}$ contains one cycle, we can temporarily consider all the bases from $B T$ as parameters, and consider the same system of linear equations for $y_{i}$ 's. Similarly, as above, at least one $y_{t}$ can be bounded. We will bound as many $y_{i}$ 's as we can. For the new periodic structure either $B T$ contains less elements or the set $C^{(2)}$ is empty.

The second part of the lemma follows from the remark that for $\mu \in T$ left multiplication of $h(\mu)$ by $h\left(r c r^{-1}\right)$, where $r$ is the path in $T$ from $v_{0}$ to the initial vertex of $\mu$, is an automorphism from $A(\Omega)$.

We call a solution $H^{+}$constructed in Lemma 6.15 a solution equivalent to $H$ with maximal number of short variables.

Consider now variables from $S$ as variables not from $\mathcal{P}^{\prime}$, so that for the equation $\Omega$ the sets $C^{(2)}$ and $B T^{\prime} \backslash B T_{0}^{\prime}$ are both empty. In this case we make the second minimal replacement, which we will describe in the lemma below. 
Definition 6.16. A pair of bases $\mu, \Delta(\mu)$ is called an overlapping pair if $\epsilon(\mu)=$ 1 and $\beta(\mu)>\alpha(\Delta(\mu))>\alpha(\mu)$ or $\epsilon(\mu)=-1$ and $\beta(\mu)<\beta(\Delta(\mu))<\alpha(\mu)$. If a closed section begins with $\alpha(\mu)$ and ends with $\beta(\Delta(\mu))$ for an overlapping pair of bases we call such a pair of bases a principal overlapping pair and say that a section is in overlapping form.

Notice, that if $\lambda \in N \mathcal{P}$, then $H(\lambda)$ is the same for any solution $H$, and we just write $\lambda$ instead of $H(\lambda)$.

LEMma 6.17. Suppose that for the generalized equation $\Omega^{\prime}$ obtained in Lemma 6.15 the sets $C^{(2)}$ and $B T^{\prime} \backslash B T_{0}^{\prime}$ are empty, $\mathcal{P}^{\prime}$ is a non-empty periodic structure, and each closed section from $\mathcal{P}^{\prime}$ has a principal overlapping pair. Then for each base $\mu \in \mathcal{P}^{\prime}$ there is a fixed presentation for $h(\mu)=\prod$ (parameters) as a product of elements $h(\lambda), \lambda \in N \mathcal{P}, h_{k} \notin \mathcal{P}^{\prime}$ corresponding to a path in $B \Gamma_{0}^{\prime}$. The maximal number of terms in this presentation is bounded.

Proof. Let $e$ be the edge in the graph $B \Gamma^{\prime}$ corresponding to a base $\mu$ and suppose $e: V \rightarrow V^{\prime}$. There is a path $s$ in $B T^{\prime}$ joining $V$ and $V^{\prime}$, and a cycle $\bar{c}$ which is a product of cycles $c(e) \in C^{(1)}$. For each $c(e)$ either $h(c(e))=1$ or $c(e)$ can be written using only edges not from $\mathcal{P}^{\prime}$; therefore, $\bar{c}$ contains only edges not from $\mathcal{P}^{\prime}$. Therefore

$$
h(\mu)=\prod(\text { parameters })=h\left(\lambda_{i_{1}}\right) \Pi_{1} \ldots h\left(\lambda_{s_{i}}\right) \Pi_{s},
$$

where the doubles of all $\lambda_{i}$ are parameters, and $\Pi_{1}, \ldots, \Pi_{s}$ are products of variables $h_{k_{i}} \notin \mathcal{P}^{\prime}$.

In the equality

$$
H(\mu)=\lambda_{i_{1}} \bar{\Pi}_{1} \ldots \lambda_{s_{i}} \bar{\Pi}_{s}
$$

where $\bar{\Pi}_{1}, \ldots, \bar{\Pi}_{s}$ are products of $H_{k_{i}}$ for variables $h_{k_{i}} \notin \mathcal{P}^{\prime}$, the cancellations between two terms in the left side are complete because the equality corresponds to a path in $B \Gamma_{0}^{\prime}$. Therefore the cancellation tree for the equality (38) can be situated on a horizontal axis with intervals corresponding to $\lambda_{i}$ 's directed either to the right or to the left. This tree can be drawn on a $P$-scaled axis. We call this one-dimensional tree a $\mu$-tree. Denote by $I(\lambda)$ the interval corresponding to $\lambda$ in the $\mu$-tree. If $I(\mu) \subseteq \bigcup_{\lambda_{i} \in N \mathcal{P}} I\left(\lambda_{i}\right)$, then we say that $\mu$ is covered by parameters. In this case a generalized equation corresponding to (38) can be situated on the intervals corresponding to bases from $N \mathcal{P}$.

We can shift the whole $\mu$-tree to the left or to the right so that in the new situation the uncovered part becomes covered by the bases from $N \mathcal{P}$. Certainly, we have to make sure that the shift is through the interval corresponding to a cycle in $C^{(1)}$. Equivalently, we can shift any base belonging to the $\mu$-tree through such an interval.

If $c$ is a shortest cycle from $C^{(1)}$, then there is a corresponding $c$-tree. Shifting this $c$-tree to the right or to the left through the intervals corresponding to $c$ bounded number of times we can cover every $H_{i}$, where $h_{i} \in S$ by a product $\lambda_{j_{1}} \bar{\Pi}_{1} \ldots \lambda_{j_{t}} \bar{\Pi}_{t}$, where $\bar{\Pi}_{1}, \ldots, \bar{\Pi}_{t}$ are products of values of variables not from $\mathcal{P}$ and $\lambda_{j_{1}}, \ldots \lambda_{j_{t}}$ are bases from $N \mathcal{P}$. Combining this covering together with the covering of $H(\mu)$ by the product (38), we obtain that $H([\alpha(\mu), \beta(\Delta(\mu))])$ is almost covered by parameters, except for the short products $\bar{\Pi}$. Let $h(\mu)$ be covered by

$$
h\left(\Lambda_{1}\right) \Pi_{1} \ldots, h\left(\Lambda_{s}\right) \Pi_{s},
$$


where $h\left(\Lambda_{1}\right), \ldots, h\left(\Lambda_{s}\right)$ are parts completely covered by parameters, and $\Pi_{1}, \ldots, \Pi_{s}$ are products of variables not in $\mathcal{P}$. We also remove those bases from $N \mathcal{P}$ from each $\Lambda_{i}$ which do not overlap with $h(\mu)$. Denote by $f_{4}$ the maximal number of bases in $N \mathcal{P}$ and $h_{i} \notin \mathcal{P}$ in the covering (39).

LEMmA 6.18. Let $\lambda_{\mu} \in N \mathcal{P}$ be a base of maximal length in the covering (39) for $\mu \in \mathcal{P}$. If for a solution $H$ of $\Omega$, and for each closed section $[\alpha(\mu), \beta(\Delta(\mu)]$ in $\mathcal{P}$, min $d(H[\alpha(\nu), \alpha(\Delta(\nu))]) \leq d\left(\lambda_{\mu}\right)$, where the minimum is taken for all pairs of overlapping bases for this section, then one can transform $\Omega$ into one of the finite number (depending on $\Omega$ ) of generalized equations $\Omega(\mathcal{P})$ which do not contain closed sections from $\mathcal{P}$ but contain the same other closed sections except for parametric sections. The content of closed sections from $\mathcal{P}$ is transferred using bases from $N \mathcal{P}$ to the parametric part. This transformation is called the second minimal replacement.

Proof. Suppose for a closed section $[\alpha(\mu), \beta(\Delta(\mu))]$ that there exists a base $\lambda$ in (39) such that $d(\lambda) \geq \min (H(\alpha(\nu), \alpha(\Delta(\nu)))$, where the minimum is taken for all pairs of overlapping bases for this section. We can shift the cover

$H\left(\Lambda_{1}\right) \bar{\Pi}_{1}, \ldots, H\left(\Lambda_{s}\right) \bar{\Pi}_{s}$ through the distance $d_{1}=d(H[\alpha(\mu), \alpha(\Delta(\mu))]$. Consider first the case when $d_{1} \leq d(\lambda)$ for the largest base in (39). Suppose the part of $H(\mu)$ corresponding to $\bar{\Pi}_{i}$ is not covered by parameters. Take the first base $\lambda_{j}$ in (39) to the right or to the left of $\bar{\Pi}_{i}$ such that $d\left(\lambda_{j}\right) \geq d_{1}$. Suppose $\lambda_{j}$ is situated to the left from $\bar{\Pi}_{i}$. Shifting $\lambda_{j}$ to the right through a bounded by $f_{4}$ multiple of $d_{1}$ we will cover $\bar{\Pi}_{i}$.

Consider now the case when $d_{1}>d(\lambda)$, but there exists an overlapping pair $\nu, \Delta(\nu)$ such that

$$
d_{2}=d(H[\alpha(\nu), \alpha(\Delta(\nu))]) \leq d(\lambda) .
$$

If the part of $H(\mu)$ corresponding to $\bar{\Pi}_{i}$ is not covered by parameters, we take the first base $\lambda_{j}$ in (39) to the right or to the left of $\bar{\Pi}_{i}$ such that $d\left(\lambda_{j}\right) \geq d_{2}$. Without loss of generality we can suppose that $\lambda_{j}$ is situated to the left of $\bar{\Pi}_{i}$. Shifting $\lambda_{j}$ to the right through a bounded by $f_{4}$ multiple of $d_{2}$ we will cover $\bar{\Pi}_{i}$.

Therefore, if the first alternative in the lemma does not take place, we can cover the whole section $[\alpha(\mu), \beta(\Delta(\mu))]$ by the bases from $N \mathcal{P}$, and transform $\Omega$ into one of the finite number of generalized equations which do not contain the closed section $[\alpha(\mu), \beta(\Delta(\mu))]$ and have all the other non-parametric sections the same. All the cancellations between two neighboring terms of any equality that we have gotten are complete, therefore the coordinate groups of new equations are quotients of $F_{R(\Omega)}$.

6.4. Proof of Proposition 6.2. The first statement follows from Lemmas 6.7 and 6.9 The second statement follows from Lemmas 6.11 6.14 6.15 6.17

\section{Elimination process: splittings of coordinate groups}

7.1. Minimal solutions. Let $F=F(A \cup B)$ be a free group with basis $A \cup B$, $\Omega$ be a generalized equation with constants from $(A \cup B)^{ \pm 1}$, and parameters $\Lambda$. Let $A(\Omega)$ be an arbitrary group of $(A \cup \Lambda)$-automorphisms of $F_{R(\Omega)}$. For solutions $H^{(1)}$ and $H^{(2)}$ of the equation $\Omega$ in the group $F$ we write $H^{(1)}<_{A(\Omega)} H^{(2)}$ if there exists an endomorphism $\pi$ of the group $F$ which is an $(A, \Lambda)$-homomorphism, and an automorphism $\sigma \in A(\Omega)$ such that the following conditions hold: (1) $\pi_{H^{(2)}}=$ 
$\sigma \pi_{H^{(1)}} \pi$, (2) For all active variables $d\left(H_{k}^{(1)}\right) \leq d\left(H_{k}^{(2)}\right)$ for all $1 \leq k \leq \rho$ and $d\left(H_{k}^{(1)}\right)<d\left(H_{k}^{(2)}\right)$ at least for one such $k$.

We also define a relation $<_{c A(\Omega)}$ by the same way as $<_{A(\Omega)}$ but with extra property: (3) for any $k, j$, if $\left(H_{k}^{(2)}\right)^{\epsilon}\left(H_{j}^{(2)}\right)^{\delta}$ in non-cancellable, then $\left(H_{k}^{(1)}\right)^{\epsilon}\left(H_{j}^{(1)}\right)^{\delta}$ in non-cancellable $(\epsilon, \delta= \pm 1)$. Obviously, both relations are transitive.

A solution $\bar{H}$ of $\Omega$ is called $A(\Omega)$-minimal if there is no any solution $\bar{H}^{\prime}$ of the equation $\Omega$ such that $\bar{H}^{\prime}<_{A(\Omega)} \bar{H}$. Since the total length $\sum_{i=1}^{\rho} l\left(H_{i}\right)$ of a solution $\bar{H}$ is a non-negative integer, every strictly decreasing chain of solutions $\bar{H}>\bar{H}^{1}>\ldots>\bar{H}^{k}>_{A(\Omega)} \ldots$ is finite. It follows that for every solution $\bar{H}$ of $\Omega$ there exists a minimal solution $\bar{H}^{0}$ such that $\bar{H}^{0}<_{A(\Omega)} \bar{H}$.

7.2. Splittings. Assign to some vertices $v$ of the tree $T(\Omega)$ splittings of $F_{R\left(\Omega_{v}\right)}$ and groups of canonical automorphisms of groups $F_{R\left(\Omega_{v}\right)}$ corresponding to these splittings.

THEOREM 7.1. (1) If $t p(v)=12$, and the quadratic equation corresponding to the quadratic section $\sigma$ is regular, then either $F_{R\left(\Omega_{v}\right)}$ is a free product where one factor is a closed surface group or there is a splitting of $F_{R\left(\Omega_{v}\right)}$ containing a $Q H$-subgroup $Q$ corresponding to this quadratic equation.

(2) If $\operatorname{tp}(v)=2$, and $\langle\mathcal{P}, R\rangle$ is a singular periodic structure, then either $F_{R\left(\Omega_{v}\right)}$ is a free product where one factor is a free abelian group or $F_{R\left(\Omega_{v}\right)}$ has a splitting with an abelian vertex group generated by the cycles $\bar{c}^{(1)}, \bar{c}^{(2)}$ and edge group generated by $\bar{c}^{(1)}$ for this periodic structure.

(3) If $t p(v)=2$, and $\langle\mathcal{P}, R\rangle$ is a non-singular non-empty periodic structure, then $F_{R\left(\Omega_{v}\right)}$ has an abelian splitting as an $H N N$ extension with stable letters corresponding to $h(e), e \in T \backslash T_{0}$.

(4) Let $7 \leq t p(v) \leq 10$. There exists a number $N=N\left(\Omega_{v}\right)$ such that if there is a branch of length $N$ in $T\left(\Omega_{v}\right)$ beginning at $v$ with each vertex $w$ of type $7 \leq t p(w) \leq 10$, then $F_{R\left(\Omega_{v}\right)}$ splits as $F_{R\left(\Omega_{v}\right)}=F_{R(\operatorname{Ker} \Omega)} * F(Z)$, where $F(Z)$ is a free group on $Z$.

(5) Let $t p(v)=15$. There exists a number $N=N\left(\Omega_{v}\right)$ such that if $T\left(\Omega_{v}\right)$ contains a branch with all vertices of type 15 beginning at $v$, then $F_{R\left(\Omega_{v}\right)}$ either has a splitting as in 3) or has a QH subgroup or both.

Moreover, all the splittings in Statements 1)-5) and the number $N$ can be found effectively.

Proof. Statement (1) follows from the argument in the description of Case 12. Statements (2) and (3) follow from Lemmas 6.7 and 6.11. Statement (4) follows from Lemma 5.2 Statement (5) follows from Proposition 7.5 to be proved below. The statement about the effectiveness follows from the effectiveness of the construction of a finite tree $T_{0}(\Omega)$ in the next two subsections.

For each vertex $v$ such that $\operatorname{tp}(v)=12$ the group of automorphisms $A\left(\Omega_{v}\right)$ assigned to it is the canonical group of automorphisms of $F_{R\left(\Omega_{v}\right)}$ corresponding to $Q$ (and, therefore, identical on $\Lambda$ ).

For each vertex $v$ such that $7 \leq t p(v) \leq 10$ we assign the group of automorphisms invariant with respect to the kernel.

For each vertex $v$ such that $t p(v)=2$, assign the group $\bar{A}_{v}$ generated by the groups of automorphisms constructed in Lemma 6.11 that applied to $\Omega_{v}$ and all possible non-singular periodic structures of this equation. 
Let $t p(v)=15$. Apply transformation $D_{3}$ and consider $\Omega=\tilde{\Omega}_{v}$. Notice that the function $\gamma_{i}$ is constant when $h_{i}$ belongs to some closed section of $\tilde{\Omega_{v}}$. Applying $D_{2}$, we can suppose that the section $[1, j+1]$ is covered exactly twice. We say now that this is a quadratic section. Assign to the vertex $v$ the group of automorphisms of $F_{R(\Omega)}$ acting identically on the non-quadratic part.

7.3. The finite subtree $T_{0}(\Omega)$ of $T(\Omega)$ : cutting off long branches. For a generalized equation $\Omega$ with parameters we construct a finite tree $T_{0}(\Omega)$. Then we show that the subtree of $T(\Omega)$ obtained by tracing those paths in $T(\Omega)$ which actually can happen for "short" solutions is a subtree of $T_{0}(\Omega)$.

According to Lemma 5.5 along an infinite path in $T(\Omega)$ one can either have $7 \leq t p\left(v_{k}\right) \leq 10$ for all $k$ or $t p\left(v_{k}\right)=12$ for all $k$, or $t p\left(v_{k}\right)=15$ for all $k$.

Lemma 7.2. [Lemma 15 from [36] Let $v_{1} \rightarrow v_{2} \rightarrow \ldots \rightarrow v_{k} \rightarrow \ldots$ be an infinite path in the tree $T(\Omega)$, and $7 \leq t p\left(v_{k}\right) \leq 10$ for all $k$. Then among $\left\{\Omega_{k}\right\}$ some generalized equation occurs infinitely many times. If $\Omega_{v_{k}}=\Omega_{v_{l}}$, then $\pi\left(v_{k}, v_{l}\right)$ is an isomorphism invariant with respect to the kernel.

LeMma 7.3. Let $t p(v)=12$. If a solution $\bar{H}$ of $\Omega_{v}$ is minimal with respect to the canonical group of automorphisms, then there is a recursive function $f_{0}$ such that in the sequence

$$
\left(\Omega_{v}, \bar{H}\right) \rightarrow\left(\Omega_{v_{1}}, \bar{H}^{1}\right) \rightarrow \ldots \rightarrow\left(\Omega_{v_{i}}, \bar{H}^{i}\right), \ldots
$$

corresponding to the path in $T\left(\Omega_{v}\right)$ and for the solution $\bar{H}$, case 12 cannot be repeated more than $f_{0}$ times.

Proof. If $\mu$ and $\Delta \mu$ both belong to the quadratic section, then $\mu$ is called a quadratic base. Consider the following set of generators for $F_{R\left(\Omega_{v}\right)}$ : variables from $\Lambda$ and quadratic bases from the active part. Relations in this set of generators consist of the following three families.

1) Relations between variables in $\Lambda$.

2) If $\mu$ is an active base and $\Delta(\mu)$ is a parametric base, and $\Delta(\mu)=h_{i} \ldots h_{i+t}$, then there is a relation $\mu=h_{i} \ldots h_{i+t}$.

3) Since $\gamma_{i}=2$ for each $h_{i}$ in the active part the product of $h_{i} \ldots h_{j}$, where $[i, j+1]$ is a closed active section, can be written in two different ways $w_{1}$ and $w_{2}$ as a product of active bases. We write the relations $w_{1} w_{2}^{-1}=1$. These relations give a quadratic system of equations with coefficients in the subgroup generated by $\Lambda$.

When we apply the entire transformation in Case 12, the number of variables is not increasing and the complexity of the generalized equation is not increasing. Suppose the same generalized equation is repeated twice in the sequence (40). for example, $\Omega_{j}=\Omega_{j+k}$. Then $\pi\left(v_{j}, v_{j+k}\right)$ is an automorphism of $F_{R\left(\Omega_{j}\right)}$ induced by the automorphism of the free product $\langle\Lambda\rangle * B$, where $B$ is a free group generated by quadratic bases, identical on $\langle\Lambda\rangle$ and fixing all words $w_{1} w_{2}^{-1}$. Therefore, $\bar{H}^{j}>$ $\bar{H}^{j+k}$, which contradicts to the minimality of $\bar{H}$. Therefore there is only a finite number (bounded by $f_{0}$ ) of possible generalized equations that can appear in the sequence (40). 
Let $\bar{H}$ be a solution of the equation $\Omega$ with quadratic part $[1, j+1]$.If $\mu$ belongs and $\Delta \mu$ does not belong to the quadratic section, then $\mu$ is called a quadraticcoefficient base. Define the following numbers:

$$
\begin{gathered}
d_{1}(\bar{H})=\sum_{i=1}^{j} d\left(H_{i}\right), \\
d_{2}(\bar{H})=\sum_{\mu} d(H[\alpha(\mu), \beta(\mu)]),
\end{gathered}
$$

where $\mu$ is a quadratic-coefficient base.

Lemma 7.4. Let $t p(v)=15$ For any solution $\bar{H}$ of $\Omega_{v}$ there is a minimal solution $\bar{H}^{+}$, which is an automorphic image of $\bar{H}$ with respect to the group of automorphisms defined in the beginning of this section, such that

$$
d_{1}\left(\bar{H}^{+}\right) \leq f_{1}\left(\Omega_{v}\right) \max \left\{d_{2}\left(\bar{H}^{+}\right), 1\right\},
$$

where $f_{1}(\Omega)$ is some recursive function.

Proof. Consider instead of $\Omega_{v}$ equation $\Omega=\left(\tilde{\Omega}_{v}\right)$ which does not have any boundary connections, $F_{R\left(\Omega_{v}\right)}$ is isomorphic to $F_{R(\Omega)}$. Consider a presentation of $F_{R\left(\Omega_{v}\right)}$ in the set of generators consisting of variables in the non-quadratic part and active bases. Relations in this generating set consist of the following three families.

1) Relations between variables in the non-quadratic part.

2) If $\mu$ is a quadratic-coefficient base and $\Delta(\mu)=h_{i} \cdots h_{i+t}$ in the nonquadratic part, then there is a relation $\mu=h_{i} \cdots h_{i+t}$.

3) Since $\gamma_{i}=2$ for each $h_{i}$ in the active part the product $h_{i} \cdots h_{j}$, where $[i, j+1]$ is a closed active section, can be written in two different ways $w_{1}$ and $w_{2}$ as a product of quadratic and quadratic-coefficient bases. We write the relations $w_{1} w_{2}^{-1}=1$.

Let $\bar{H}$ be a solution of $\Omega_{v}$ minimal with respect to the canonical group of automorphisms of the free product $B_{1} * B$, where $B$ is a free group generated by quadratic bases, and $B_{1}$ is a subgroup of $F_{R\left(\Omega_{v}\right)}$ generated by variables in the non-quadratic part, identical on $\langle\Lambda\rangle$ and fixing all words $w_{1} w_{2}^{-1}$.

Consider the sequence

$$
(\Omega, \bar{H}) \rightarrow\left(\Omega_{v_{1}}, \bar{H}^{1}\right) \rightarrow \ldots \rightarrow\left(\Omega_{v_{i}}, \bar{H}^{i}\right), \ldots
$$

Apply now the entire transformations to the quadratic section of $\Omega$. As in the proof of the previous lemma, each time we apply the entire transformation, we do not increase complexity and do not increase the total number of items in the whole interval. Since $\bar{H}$ is a solution of $\Omega_{v}$, if the same generalized equation appear in this sequence $2^{4^{j^{2}}}+1$ times then for some $j, j+k$ we have $\bar{H}^{j}>_{c} \bar{H}^{j+k}$, therefore the same equation can only appear a bounded number of times. Every quadratic base (except those that become matching bases of length 1) in the quadratic part can be transferred to the non-quadratic part with the use of some quadratic-coefficient base as a carrier base. This means that the length of the transferred base is equal to the length of the part of the quadratic-coefficient carrier base, which will then be deleted. The double of the transferred base becomes a quadratic-coefficient base. Because there are not more than $n_{A}$ bases in the active part, this would give

$$
d_{1}\left(\bar{H}^{\prime}\right) \leq n_{A} d_{2}\left(\bar{H}^{\prime}\right),
$$


for some solution $\bar{H}^{+}$of the equation $\tilde{\Omega}_{v}$. But $\bar{H}^{+}$is obtained from the minimal solution $\bar{H}$ in a bounded number of steps.

We call a path $v_{1} \rightarrow v_{2} \rightarrow \ldots \rightarrow v_{k} \rightarrow \ldots$ in $T(\Omega)$ for which $7 \leq t p\left(v_{k}\right) \leq 10$ for all $k$ or type 12 prohibited if some generalized equation with $\rho$ variables occurs among $\left\{\Omega_{v_{i}} \mid 1 \leq i \leq \ell\right\}$ at least $2^{\left(4 \rho^{2}\right)}+1$ times. We will define below also prohibited paths in $T(\Omega)$, for which $t p\left(v_{k}\right)=15$ for all $k$. We will need some auxiliary definitions.

Introduce a new parameter

$$
\tau_{v}^{\prime}=\tau_{v}+\rho-\rho_{v}^{\prime},
$$

where $\rho$ is the number of variables of the initial equation $\Omega$ and $\rho_{v}^{\prime}$ the number of free variables belonging to the non-active sections of the equation $\Omega_{v}$. We have $\rho_{v}^{\prime} \leq \rho$ (see the proof of Lemma [5.5), hence $\tau_{v}^{\prime} \geq 0$. In addition if $v_{1} \rightarrow v_{2}$ is an auxiliary edge, then $\tau_{2}^{\prime}<\tau_{1}^{\prime}$.

Define by the joint induction on $\tau_{v}^{\prime}$ a finite subtree $T_{0}\left(\Omega_{v}\right)$ and a natural number $s\left(\Omega_{v}\right)$. The tree $T_{0}\left(\Omega_{v}\right)$ will have $v$ as a root and consist of some vertices and edges of $T(\Omega)$ that lie higher than $v$. Let $\tau_{v}^{\prime}=0$; then in $T(\Omega)$ there can not be auxiliary edges and vertices of type 15 higher than $v$. Hence a subtree $T_{0}\left(\Omega_{v}\right)$ consisting of vertices $v_{1}$ of $T(\Omega)$ that are higher than $v$, and for which the path from $v$ to $v_{1}$ does not contain prohibited subpaths, is finite.

Let now

$$
s\left(\Omega_{v}\right)=\max _{w} \max _{\langle\mathcal{P}, R\rangle}\left\{\rho_{w} f_{2}\left(\Omega_{w}, \mathcal{P}, R\right), f_{4}\left(\Omega_{w}^{\prime}, \mathcal{P}, R\right)\right\},
$$

where $w$ runs through all the vertices of $T_{0}(v)$ for which $t p(w)=2, \Omega_{w}$ contains nontrivial non-parametric sections, $\langle\mathcal{P}, R\rangle$ is the set of non-singular periodic structures of the equation $\tilde{\Omega}_{w}, f_{2}$ is a function appearing in Lemma $6.9\left(f_{2}\right.$ is present only if a periodic structure has empty set $N \mathcal{P})$ and $\Omega_{w}^{\prime}$ is constructed as in Lemma 6.15. where $f_{4}$ is a function appearing in covering 39.

Suppose now that $\tau_{v}^{\prime}>0$ and that for all $v_{1}$ with $\tau_{v_{1}}^{\prime}<\tau_{v}^{\prime}$ the tree $T_{0}\left(\Omega_{v_{1}}\right)$ and the number $s\left(\Omega_{v_{1}}\right)$ are already defined. We begin with the consideration of the paths

$$
r=v_{1} \rightarrow v_{2} \rightarrow \ldots \rightarrow v_{m},
$$

where $t p\left(v_{i}\right)=15(1 \leq i \leq m)$. We have $\tau_{v_{i}}^{\prime}=\tau_{v}^{\prime}$.

Denote by $\mu_{i}$ the carrier base of the equation $\Omega_{v_{i}}$. The path (45) will be called $\mu$-reducing if $\mu_{1}=\mu$ and either there are no auxiliary edges from the vertex $v_{2}$ and $\mu$ occurs in the sequence $\mu_{1}, \ldots, \mu_{m-1}$ at least twice, or there are auxiliary edges $v_{2} \rightarrow w_{1}, v_{2} \rightarrow w_{2} \ldots, v_{2} \rightarrow w_{k}$ from $v_{2}$ and $\mu$ occurs in the sequence $\mu_{1}, \ldots, \mu_{m-1}$ at least $\max _{1 \leq i \leq k} s\left(\Omega_{w_{i}}\right)$ times.

The path (45) will be called prohibited, if it can be represented in the form

$$
r=r_{1} s_{1} \ldots r_{l} s_{l} r^{\prime},
$$

such that for some sequence of bases $\eta_{1}, \ldots, \eta_{l}$ the following three properties hold:

1) every base occurring at least once in the sequence $\mu_{1}, \ldots, \mu_{m-1}$ occurs at least $40 n^{2} f_{1}\left(\Omega_{v_{2}}\right)+20 n+1$ times in the sequence $\eta_{1}, \ldots, \eta_{l}$, where $n$ is the number of pairs of bases in equations $\Omega_{v_{i}}$,

2) the path $r_{i}$ is $\eta_{i}$-reducing; 
3) every transfer base of some equation of path $r$ is a transfer base of some equation of path $r^{\prime}$.

The property of path (45) of being prohibited is algorithmically decidable. Every infinite path (45) contains a prohibited subpath. Indeed, let $\omega$ be the set of all bases occurring in the sequence $\mu_{1}, \ldots, \mu_{m}, \ldots$ infinitely many times, and $\tilde{\omega}$ the set of all bases, that are transfer bases of infinitely many equations $\Omega_{v_{i}}$. If one cuts out some finite part in the beginning of this infinite path, one can suppose that all the bases in the sequence $\mu_{1}, \ldots, \mu_{m}, \ldots$ belong to $\omega$ and each base that is a transfer base of at least one equation, belongs to $\tilde{\omega}$. Such an infinite path for any $\mu \in \omega$ contains infinitely many non-intersecting $\mu$-reducing finite subpaths. Hence it is possible to construct a subpath (46) of this path satisfying the first two conditions in the definition of a prohibited subpath. Making $r^{\prime}$ longer, one obtains a prohibited subpath.

Let $T^{\prime}\left(\Omega_{v}\right)$ be a subtree of $T\left(\Omega_{v}\right)$ consisting of the vertices $v_{1}$ for which the path from $v$ to $v_{1}$ in $T(\Omega)$ contains neither prohibited subpaths nor vertices $v_{2}$ with $\tau_{v_{2}}^{\prime}<\tau_{v}^{\prime}$, except perhaps $v_{1}$. So the terminal vertices of $T^{\prime}\left(\Omega_{v}\right)$ are either vertices $v_{1}$ such that $\tau_{v_{1}}^{\prime}<\tau_{v}^{\prime}$, or terminal vertices of $T\left(\Omega_{v}\right)$. A subtree $T^{\prime}\left(\Omega_{v}\right)$ can be effectively constructed. $T_{0}\left(\Omega_{v}\right)$ is obtained by attaching of $T_{0}\left(\Omega_{v_{1}}\right)$ (already constructed by the induction hypothesis) to those terminal vertices $v_{1}$ of $T^{\prime}\left(\Omega_{v}\right)$ for which $\tau_{v_{1}}^{\prime}<\tau_{v}^{\prime}$. The function $s\left(\Omega_{v}\right)$ is defined by (44). Let now $T_{0}(\Omega)=T_{0}\left(\Omega_{v_{0}}\right)$. This tree is finite by construction.

7.4. Paths corresponding to minimal solutions of $\Omega$ are in $T_{0}(\Omega)$. Notice, that if $t p(v) \geq 6$ and $v \rightarrow w_{1}, \ldots, v \rightarrow w_{m}$ is the list of principal outgoing edges from $v$, then the generalized equations $\Omega_{w_{1}}, \ldots, \Omega_{w_{m}}$ are obtained from $\Omega_{v}$ by the application of several elementary transformations. Denote by $e$ a function that assigns a pair $\left(\Omega_{w_{i}}, \bar{H}^{(i)}\right)$ to the pair $\left(\Omega_{v}, \bar{H}\right)$. For $t p(v)=4,5$ this function is identical.

If $t p(v)=15$ and there are auxiliary edges from the vertex $v$, then the carrier base $\mu$ of the equation $\Omega_{v}$ intersects $\Delta(\mu)$. For any solution $\bar{H}$ of the equation $\Omega_{v}$ one can construct a solution $\bar{H}^{\prime}$ of the equation $\Omega_{v^{\prime}}$ by $H_{\rho_{v}+1}^{\prime}=H[1, \beta(\Delta(\mu))]$. Let $e^{\prime}\left(\Omega_{v}, \bar{H}\right)=e\left(\Omega_{v^{\prime}}, \bar{H}^{\prime}\right)$.

In the beginning of this section we assigned to vertices $v$ of type 12, 15, 2 and such that $7 \leq t p(v) \leq 10$ of $T(\Omega)$ the groups of automorphisms $A\left(\Omega_{v}\right)$. Denote by Aut $(\Omega)$ the group of automorphisms of $F_{R(\Omega)}$, generated by all groups $\pi\left(v_{0}, v\right) A\left(\Omega_{v}\right) \pi\left(v_{0}, v\right)^{-1}, v \in T_{0}(\Omega)$. (Here $\pi\left(v_{0}, v\right)$ is an isomorphism, because $t p(v) \neq 1$.) We are to formulate the main technical result of this section. The following proposition states that every minimal solution of a generalized equation $\Omega$ with respect to the group $A(\Omega)$ either factors through one of the finite family of proper quotients of the group $F_{R(\Omega)}$ or (in the case of a non-empty parametric part) can be transferred to the parametric part.

Proposition 7.5. For any solution $\bar{H}$ of a generalized equation $\Omega$ there exists a terminal vertex $w$ of the tree $T_{0}(\Omega)$ having type 1 or 2 , and a solution $\bar{H}^{(w)}$ of a generalized equation $\Omega_{w}$ such that

(1) $\pi_{\bar{H}}=\sigma \pi\left(v_{0}, w\right) \pi_{\bar{H}^{(w)}} \pi$ where $\pi$ is an endomorphism of a free group $F$ $\sigma \in \operatorname{Aut}(\Omega)$

(2) if $t p(w)=2$ and the equation $\Omega_{w}$ contains nontrivial non-parametric sections, then there exists a primitive cyclically reduced word $P$ such that 
$\bar{H}^{(w)}$ is periodic with respect to $\mathcal{P}$ and one of the following conditions holds:

(a) the equation $\Omega_{w}$ is singular with respect to a periodic structure $\mathcal{P}\left(\bar{H}^{(w)}, P\right)$ and the first minimal replacement can be applied,

(b) it is possible to apply the second minimal replacement and make the family of closed sections in $\mathcal{P}$ empty.

Construct a directed tree with paths from the initial vertex

$$
(\Omega, \bar{H})=\left(\Omega_{v_{0}}, \bar{H}^{(0)}\right) \rightarrow\left(\Omega_{v_{1}}, \bar{H}^{(1)}\right) \rightarrow \ldots \rightarrow\left(\Omega_{v_{u}}, \bar{H}^{(u)}\right) \rightarrow \ldots
$$

in which the $v_{i}$ are the vertices of the tree $T(\Omega)$ in the following way. Let $v_{1}=v_{0}$ and let $\bar{H}^{(1)}$ be some solution of the equation $\Omega$, minimal with respect to the group of automorphisms $A\left(\Omega v_{0}\right)$ with the property $\bar{H} \geq \bar{H}^{(1)}$.

Let $i \geq 1$ and suppose the term $\left(\Omega_{v_{i}}, \bar{H}^{(i)}\right)$ of the sequence (47) has been already constructed. If $7 \leq t p\left(v_{i}\right) \leq 10$ or $t p\left(v_{i}\right)=12$ and there exists a minimal solution $\bar{H}^{+}$of $\Omega_{v_{i}}$ such that $\bar{H}^{+}<\bar{H}^{(i)}$, then we set $v_{i+1}=v_{i}, \bar{H}^{(i+1)}=\bar{H}^{+}$.

If $t p\left(v_{i}\right)=15, v_{i} \neq v_{i-1}$ and there are auxiliary edges from vertex $v_{i}: v_{i} \rightarrow$ $w_{1}, \ldots, v_{i} \rightarrow w_{k}$ (the carrier base $\mu$ intersects with its double $\Delta(\mu)$ ), then there exists a primitive word $P$ such that

$$
H^{(i)}[1, \beta(\Delta(\mu))] \equiv P^{r} P_{1}, r \geq 2, P \equiv P_{1} P_{2},
$$

where $\equiv$ denotes a graphical equality. In this case the path (47) can be continued along several possible edges of $T(\Omega)$.

For each group of automorphisms assigned to vertices of type 2 in the trees $T_{0}\left(\Omega_{w_{i}}\right), i=1, \ldots, k$ and non-singular periodic structure including the closed section $\left[1, \beta(\Delta(\mu)]\right.$ of the equation $\Omega_{v_{i}}$ and corresponding to solution $\bar{H}^{(i)}$ we replace $\bar{H}^{(i)}$ by a solution $\bar{H}^{(i)+}$ with maximal number of short variables (see the definition after Lemma 6.15). This collection of short variables can be different for different periodic structures. Either all the variables in $\bar{H}^{(i)+}$ are short or there exists a parametric base $\lambda_{\max }$ of maximal length in the covering [39] Suppose there is a $\mu$ reducing path (45) beginning at $v_{i}$ and corresponding to $\bar{H}^{(i)+}$. Let $\mu_{1}, \ldots, \mu_{m}$ be the leading bases of this path. Let $\tilde{H}^{1}=H^{(i)+}, \ldots, \tilde{H}^{j}$ be solutions of the generalized equations corresponding to the vertices of this path. If for some $\mu_{i}$ there is an inequality $d\left(\tilde{H}^{j}\left[\alpha\left(\mu_{i}\right), \alpha\left(\Delta\left(\mu_{i}\right)\right)\right]\right) \leq d\left(\lambda_{\max }\right)$, we set $\left(\Omega_{v_{i+1}}, \bar{H}^{(i+1)}\right)=e^{\prime}\left(\Omega_{v_{i}}, \bar{H}^{(i)}\right)$ and call the section $[1, \beta(\Delta(\mu))]$ which becomes non-active, potentially transferable.

If there is a singular periodic structure in a vertex of type 2 of some tree $T_{0}\left(\Omega_{w_{i}}\right), i \in\{1, \ldots, k\}$, including the closed section $\left[1, \beta(\Delta(\mu)]\right.$ of the equation $\Omega_{v_{i}}$ and corresponding to the solution $\bar{H}^{(i)}$, we also include the possibility $\left(\Omega_{v_{i+1}}, \bar{H}^{(i+1)}\right)=e^{\prime}\left(\Omega_{v_{i}}, \bar{H}^{(i)}\right)$.

In all of the other cases we set $\left(\Omega_{v_{i+1}}, \bar{H}^{(i+1)}\right)=e\left(\Omega_{v_{i}}, \bar{H}^{(i)+}\right)$, where $\bar{H}^{(i)+}$ is a solution with maximal number of short variables and minimal solution of $\Omega_{v_{i}}$ with respect to the canonical group of automorphisms $P_{v_{i}}$ (if it exists). The path (47) ends if $t p\left(v_{i}\right) \leq 2$.

We will show that in the path (47) $v_{i} \in T_{0}(\Omega)$. We use induction on $\tau^{\prime}$. Suppose $v_{i} \notin T_{0}(\Omega)$, and let $i_{0}$ be the first of such numbers. It follows from the construction of $T_{0}(\Omega)$ that there exists $i_{1}<i_{0}$ such that the path from $v_{i_{1}}$ into $v_{i_{0}}$ contains a subpath prohibited in the construction of $T_{2}\left(\Omega_{v_{i_{1}}}\right)$. From the minimality of $i_{0}$ it follows that this subpath goes from $v_{i_{2}} \quad\left(i_{1} \leq i_{2}<i_{0}\right)$ to $v_{i_{0}}$. It cannot be that $7 \leq t p\left(v_{i}\right) \leq 10$ or $\operatorname{tp}\left(v_{i}\right)=12$ for all $i_{2} \leq i \leq i_{1}$, because there will 
be two indices $p<q$ between $i_{2}$ and $i_{0}$ such that $\bar{H}^{(p)}=\bar{H}^{(q)}$, and this gives a contradiction, because in this case it must be by construction $v_{p+1}=v_{p}$. So $\operatorname{tp}\left(v_{i}\right)=15\left(i_{2} \leq i \leq i_{0}\right)$.

Suppose we have a subpath (45) corresponding to the fragment

$$
\left(\Omega_{v_{1}}, \bar{H}^{(1)}\right) \rightarrow\left(\Omega_{v_{2}}, \bar{H}^{(2)}\right) \rightarrow \ldots \rightarrow\left(\Omega_{v_{m}}, \bar{H}^{(m)}\right) \rightarrow \ldots
$$

of the sequence (47). Here $v_{1}, v_{2}, \ldots, v_{m-1}$ are vertices of the tree $T_{0}(\Omega)$, and for all vertices $v_{i}$ the edge $v_{i} \rightarrow v_{i+1}$ is principal.

As before, let $\mu_{i}$ denote the carrier base of $\Omega_{v_{i}}$, and $\omega=\left\{\mu_{1}, \ldots, \mu_{m-1}\right\}$, and $\tilde{\omega}$ denote the set of such bases which are transfer bases for at least one equation in (49). By $\omega_{1}$ denote the set of such bases $\mu$ for which either $\mu$ or $\Delta(\mu)$ belongs to $\omega \cup \tilde{\omega}$; by $\omega_{2}$ denote the set of all the other bases. Let

$$
\alpha(\omega)=\min \left(\min _{\mu \in \omega_{2}} \alpha(\mu), j\right),
$$

where $j$ is the boundary between active and non-active sections. Let $X_{\mu} \stackrel{\circ}{=} H[\alpha(\mu), \beta(\mu)]$. If $(\Omega, \bar{H})$ is a member of sequence (49), then denote

$$
\begin{gathered}
d_{\omega}(\bar{H})=\sum_{i=1}^{\alpha(\omega)-1} d\left(H_{i}\right), \\
\psi_{\omega}(\bar{H})=\sum_{\mu \in \omega_{1}} d\left(X_{\mu}\right)-2 d_{\omega}(\bar{H}) .
\end{gathered}
$$

Every item $h_{i}$ of the section $[1, \alpha(\omega)]$ belongs to at least two bases, and both bases are in $\omega_{1}$, hence $\psi_{\omega}(\bar{H}) \geq 0$.

Consider the quadratic part of $\tilde{\Omega}_{v_{1}}$ which is situated to the left of $\alpha(\omega)$. The solution $\bar{H}^{(1)}$ is minimal with respect to the canonical group of automorphisms corresponding to this vertex. By Lemma 7.4 we have

$$
d_{1}\left(\bar{H}^{(1)}\right) \leq f_{1}\left(\Omega_{v_{1}}\right) d_{2}\left(\bar{H}^{(1)}\right) .
$$

Using this inequality we estimate the length of the interval participating in the process $d_{\omega}\left(\bar{H}^{(1)}\right)$ from above by a product of $\psi_{\omega}$ and some function depending on $f_{1}$. This will be inequality [57] Then we will show that for a prohibited subpath the length of the participating interval must be reduced by more than this figure (equalities 67 681). This will imply that there is no prohibited subpath in the path 49

Denote by $\gamma_{i}(\omega)$ the number of bases $\mu \in \omega_{1}$ containing $h_{i}$. Then

$$
\sum_{\mu \in \omega_{1}} d\left(X_{\mu}^{(1)}\right)=\sum_{i=1}^{\rho} d\left(H_{i}^{(1)}\right) \gamma_{i}(\omega),
$$

where $\rho=\rho\left(\Omega_{v_{1}}\right)$. Let $I=\left\{i \mid 1 \leq i \leq \alpha(\omega)-1 \& \gamma_{i}=2\right\}$ and $J=\{i \mid 1 \leq i \leq$ $\left.\alpha(\omega)-1 \& \gamma_{i}>2\right\}$. By (50)

$$
d_{\omega}\left(\bar{H}^{(1)}\right)=\sum_{i \in I} d\left(H_{i}^{(1)}\right)+\sum_{i \in J} d\left(H_{i}^{(1)}\right)=d_{1}\left(\bar{H}^{(1)}\right)+\sum_{i \in J} d\left(H_{i}^{(1)}\right) .
$$

Let $(\lambda, \Delta(\lambda))$ be a pair of quadratic-coefficient bases of the equation $\tilde{\Omega}_{v_{1}}$, where $\lambda$ belongs to the nonquadratic part. This pair can appear only from the bases $\mu \in \omega_{1}$. There are two types of quadratic-coefficient bases. 
Type 1. $\lambda$ is situated to the left of the boundary $\alpha(\omega)$. Then $\lambda$ is formed by items $\left\{h_{i} \mid i \in J\right\}$ and hence $d\left(X_{\lambda}\right) \leq \sum_{i \in J} d\left(H_{i}^{(1)}\right)$. Thus the sum of the lengths $d\left(X_{\lambda}\right)+d\left(X_{\Delta(\lambda)}\right)$ for quadratic-coefficient bases of this type is not more than $2 n \sum_{i \in J} d\left(H_{i}^{(1)}\right)$.

Type 2. $\lambda$ is situated to the right of the boundary $\alpha(\omega)$. The sum of length of the quadratic-coefficient bases of the second type is not more than $2 \sum_{i=\alpha(\omega)}^{\rho} d\left(H_{i}^{(1)}\right) \gamma_{i}(\omega)$.

We have

$$
d_{2}\left(\bar{H}^{(1)}\right) \leq 2 n \sum_{i \in J} d\left(H_{i}^{(1)}\right)+2 \sum_{i=\alpha(\omega)}^{\rho} d\left(H_{i}^{(1)}\right) \gamma_{i}(\omega) .
$$

Now (51) and (53) imply

$$
\psi_{\omega}\left(\bar{H}_{i}^{(1)}\right) \geq \sum_{i \in J} d\left(H_{i}^{(1)}\right)+\sum_{i=\alpha(\omega)}^{\rho} d\left(H_{i}^{(1)}\right) \gamma_{i}(\omega) .
$$

Inequalities (52), (54),(55), (156) imply

$$
d_{\omega}\left(\bar{H}^{(1)}\right) \leq \max \left\{\psi_{\omega}\left(\bar{H}^{(1)}\right)\left(2 n f_{1}\left(\Omega_{v_{1}}\right)+1\right), f_{1}\left(\Omega_{v_{1}}\right)\right\}
$$

From the definition of Case 15 it follows that all the words $H^{(i)}\left[1, \rho_{i}+1\right]$ are the ends of the word $H^{(1)}\left[1, \rho_{1}+1\right]$, that is

$$
H^{(1)}\left[1, \rho_{1}+1\right] \doteq U_{i} H^{(i)}\left[1, \rho_{i}+1\right] .
$$

On the other hand bases $\mu \in \omega_{2}$ participate in these transformations neither as carrier bases nor as transfer bases; hence $H^{(1)}\left[\alpha(\omega), \rho_{1}+1\right]$ is the end of the word $H^{(i)}\left[1, \rho_{i}+1\right]$, that is

$$
H^{(i)}\left[1, \rho_{i}+1\right] \doteq V_{i} H^{(1)}\left[\alpha(\omega), \rho_{1}+1\right] .
$$

So we have

$$
d_{\omega}\left(\bar{H}^{(i)}\right)-d_{\omega}\left(\bar{H}^{(i+1)}\right)=d\left(V_{i}\right)-d\left(V_{i+1}\right)=d\left(U_{i+1}\right)-d\left(U_{i}\right)=d\left(X_{\mu_{i}}^{(i)}\right)-d\left(X_{\mu_{i}}^{(i+1)}\right) .
$$

In particular (51), (60) imply that $\psi_{\omega}\left(\bar{H}^{(1)}\right)=\psi_{\omega}\left(\bar{H}^{(2)}\right)=\ldots \psi_{\omega}\left(\bar{H}^{(m)}\right)=\psi_{\omega}$. Denote the number (60) by $\delta_{i}$.

Let the path (45) be $\mu$-reducing, that is either $\mu_{1}=\mu$ and $v_{2}$ does not have auxiliary edges and $\mu$ occurs in the sequence $\mu_{1}, \ldots, \mu_{m-1}$ at least twice, or $v_{2}$ does have auxiliary edges $v_{2} \rightarrow w_{1}, \ldots v_{2} \rightarrow w_{k}$ and the base $\mu$ occurs in the sequence $\mu_{1}, \ldots, \mu_{m-1}$ at least $\max _{1 \leq i \leq k} s\left(\Omega_{w_{i}}\right)$ times. Estimate $d\left(U_{m}\right)=\sum_{i=1}^{m-1} \delta_{i}$ from below. First notice that if $\mu_{i_{1}}=\mu_{i_{2}}=\mu\left(i_{1}<i_{2}\right)$ and $\mu_{i} \neq \mu$ for $i_{1}<i<i_{2}$, then

$$
\sum_{i=i_{1}}^{i_{2}-1} \delta_{i} \geq d\left(H^{i_{1}+1}\left[1, \alpha\left(\Delta\left(\mu_{i_{1}+1}\right)\right)\right]\right) .
$$

Indeed, if $i_{2}=i_{1}+1$, then $\delta_{i_{1}}=d\left(H^{\left(i_{1}\right)}[1, \alpha(\Delta(\mu))]=d\left(H^{\left(i_{1}+1\right)}[1, \alpha(\Delta(\mu))]\right.\right.$. If $i_{2}>i_{1}+1$, then $\mu_{i_{1}+1} \neq \mu$ and $\mu$ is a transfer base in the equation $\Omega_{v_{i_{1}+1}}$. Hence $\delta_{i_{1}+1}+d\left(H^{\left(i_{1}+2\right)}[1, \alpha(\mu)]\right)=d\left(H^{\left(i_{1}+1\right)}\left[1, \alpha\left(\mu_{i_{1}+1}\right)\right]\right)$. Now f61) follows from

$$
\sum_{i=i_{1}+2}^{i_{2}-1} \delta_{i} \geq d\left(H^{\left(i_{1}+2\right)}[1, \alpha(\mu)]\right)
$$


So if $v_{2}$ does not have outgoing auxiliary edges, that is the bases $\mu_{2}$ and $\Delta\left(\mu_{2}\right)$ do not intersect in the equation $\Omega_{v_{2}}$; then (61) implies that

$$
\sum_{i=1}^{m-1} \delta_{i} \geq d\left(H^{(2)}\left[1, \alpha\left(\Delta \mu_{2}\right)\right]\right) \geq d\left(X_{\mu_{2}}^{(2)}\right) \geq d\left(X_{\mu}^{(2)}\right)=d\left(X_{\mu}^{(1)}\right)-\delta_{1},
$$

which implies that

$$
\sum_{i=1}^{m-1} \delta_{i} \geq \frac{1}{2} d\left(X_{\mu}^{(1)}\right)
$$

Suppose now there are outgoing auxiliary edges from the vertex $v_{2}: v_{2} \rightarrow$ $w_{1}, \ldots, v_{2} \rightarrow w_{k}$. The equation $\Omega_{v_{1}}$ has some solution. Let $H^{(2)}\left[1, \alpha\left(\Delta\left(\mu_{2}\right)\right)\right] \doteq$ $Q$, and $P$ a word (in the final $h$ 's) such that $Q \doteq P^{d}$, then $X_{\mu_{2}}^{(2)}$ and $X_{\mu}^{(2)}$ are beginnings of the word $H^{(2)}\left[1, \beta\left(\Delta\left(\mu_{2}\right)\right)\right]$, which is a beginning of $P^{\infty}$. Denote $M=\max _{1 \leq j \leq k} s\left(\Omega_{w_{j}}\right)$.

By the construction of (47) we either have

$$
X_{\mu}^{(2)} \doteq P^{r} P_{1}, P \doteq P_{1} P_{2}, r<M .
$$

or for each base $\mu_{i}, i \geq 2$, there is an inequality $d\left(H^{(i)}\left(\alpha\left(\mu_{i}\right), \alpha\left(\Delta\left(\mu_{i}\right)\right)\right)\right) \geq d(\lambda)$ and therefore

$$
d\left(X_{\mu}^{(2)}\right)<M d\left(H^{(i)}\left[\alpha\left(\mu_{i}\right), \alpha\left(\Delta\left(\mu_{i}\right)\right)\right]\right) .
$$

Let $\mu_{i_{1}}=\mu_{i_{2}}=\mu ; i_{1}<i_{2} ; \mu_{i} \neq \mu$ for $i_{1}<i<i_{2}$. If

$$
d\left(X_{\mu_{i_{1}+1}}^{\left(i_{1}+1\right)}\right) \geq 2 d(P)
$$

and $H^{\left(i_{1}+1\right)}\left[1, \rho_{i_{1}+1}+1\right]$ begins with a cyclic permutation of $P^{3}$, then

$d\left(H^{\left(i_{1}+1\right)}\left[1, \alpha\left(\Delta\left(\mu_{i_{1}+1}\right)\right)\right]\right)>d\left(X_{\mu}^{(2)}\right) / M$. Together with (61) this gives $\sum_{i=i_{1}}^{i_{2}-1} \delta_{i}>$ $d\left(X_{\mu}^{(2)}\right) / M$. The base $\mu$ occurs in the sequence $\mu_{1}, \ldots, \mu_{m-1}$ at least $M$ times, so either 650ils for some $i_{1} \leq m-1$ or $\sum_{i=1}^{m-1} \delta_{i}(M-3) d\left(X_{\mu}^{(2)}\right) / M$.

If (65) fails, then the inequality $d\left(X_{\mu_{i}}^{(i+1)}\right) \leq d\left(X_{\mu_{i+1}}^{(i+1)}\right)$, and the definition 600 imply that

$$
\sum_{i=1}^{i_{1}} \delta_{i} \geq d\left(X_{\mu}^{(1)}\right)-d\left(X_{\mu_{i_{1}+1}}^{\left(i_{1}+1\right)}\right) \geq(M-2) d\left(X_{\mu}^{(2)}\right) / M ;
$$

so everything is reduced to the second case.

Let

$$
\sum_{i=1}^{m-1} \delta_{i} \geq(M-3) d\left(X_{\mu}^{(1)}\right) / M .
$$

Notice that 61) implies for $i_{1}=1 \sum_{i=1}^{m-1} \delta_{i} \geq d(Q) \geq d(P)$; so $\sum_{i=1}^{m-1} \delta_{i} \geq$ $\max \{1, M-3\} d\left(X_{\mu}^{(2)}\right) / M$. Together with (63) this implies $\sum_{i=1}^{m-1} \delta_{i} \geq \frac{1}{5} d\left(X_{\mu}^{(2)}\right)=$ $\frac{1}{5}\left(d\left(X_{\mu}^{(1)}\right)-\delta_{1}\right)$. Finally,

$$
\sum_{i=1}^{m-1} \delta_{i} \geq \frac{1}{10} d\left(X_{\mu}^{(1)}\right)
$$

Comparing (62) and (66) we can see that for the $\mu$-reducing path (45) inequality (66) always holds. 
Suppose now that the path (45) is prohibited; hence it can be represented in the form (46). From definition (51) we have $\sum_{\mu \in \omega_{1}} d\left(X_{\mu}^{(m)}\right) \geq \psi_{\omega}$; so at least for one base $\mu \in \omega_{1}$ the inequality $d\left(X_{\mu}^{(m)}\right) \geq \frac{1}{2 n} \psi_{\omega}$ holds. Because $X_{\mu}^{(m)} \doteq\left(X_{\Delta(\mu)}^{(m)}\right)^{ \pm 1}$, we can suppose that $\mu \in \omega \cup \tilde{\omega}$. Let $m_{1}$ be the length of the path $r_{1} s_{1} \ldots r_{l} s_{l}$ in (46). If $\mu \in \tilde{\omega}$ then by the third part of the definition of a prohibited path there exists $m_{1} \leq i \leq m$ such that $\mu$ is a transfer base of $\Omega_{v_{i}}$. Hence, $d\left(X_{\mu_{i}}^{\left(m_{1}\right)}\right) \geq d\left(X_{\mu_{i}}^{(i)}\right) \geq$ $d\left(X_{\mu}^{(i)}\right) \geq d\left(X_{\mu}^{(m)}\right) \geq \frac{1}{2 n} \psi_{\omega}$. If $\mu \in \omega$, then take $\mu$ instead of $\mu_{i}$. We proved the existence of a base $\mu \in \omega$ such that

$$
d\left(X_{\mu}^{\left(m_{1}\right)}\right) \geq \frac{1}{2 n} \psi_{\omega}
$$

By the definition of a prohibited path, the inequality $d\left(X_{\mu}^{(i)}\right) \geq d\left(X_{\mu}^{\left(m_{1}\right)}\right)(1 \leq i \leq$ $m_{1}$ ), (66), and (67) we obtain

$$
\sum_{i=1}^{m_{1}-1} \delta_{i} \geq \max \left\{\frac{1}{20 n} \psi_{\omega}, 1\right\}\left(40 n^{2} f_{1}+20 n+1\right) .
$$

By (60) the sum in the left part of the inequality (68) equals $d_{\omega}\left(\bar{H}^{(1)}\right)-$ $d_{\omega}\left(\bar{H}^{\left(m_{1}\right)}\right)$; hence

$$
d_{\omega}\left(\bar{H}^{(1)}\right) \geq \max \left\{\frac{1}{20 n} \psi_{\omega}, 1\right\}\left(40 n^{2} f_{1}+20 n+1\right),
$$

which contradicts (57).

This contradiction was obtained from the supposition that there are prohibited paths (49) in the path (47). Hence (47) does not contain prohibited paths. This implies that $v_{i} \in T_{0}(\Omega)$ for all $v_{i}$ in (47). For all $i v_{i} \rightarrow v_{i+1}$ is an edge of a finite tree. Hence the path (47) is finite. Let $\left(\Omega_{w}, \bar{H}^{w}\right)$ be the final term of this sequence. We show that $\left(\Omega_{w}, \bar{H}^{w}\right)$ satisfies all the properties formulated in the lemma.

The first property is obvious.

Let $t p(w)=2$ and let $\Omega_{w}$ have non-trivial non-parametric part. It follows from the construction of (47) that if $[j, k]$ is a non-active section for $\Omega_{v_{i}}$ then $H^{(i)}[j, k] \doteq$ $H^{(i+1)}[j, k] \doteq \ldots H^{(w)}[j, k]$. Hence (48) and the definition of $s\left(\Omega_{v}\right)$ imply that the word $h_{1} \ldots h_{\rho_{w}}$ can be subdivided into subwords $h\left[i_{1}, i_{2}\right], \ldots, h\left[i_{k-1}, i_{k}\right]$, such that for any $a$ either $H^{(w)}$ has length 1 , or $h\left[i_{a}, i_{a+1}\right]$ does not participate in basic and coefficient equations, or $H^{(w)}\left[i_{a}, i_{a+1}\right]$ can be written as

$$
H^{(w)}\left[i_{a}, i_{a+1}\right] \doteq P_{a}^{r} P_{a}^{\prime} ; P_{a} \doteq P_{a}^{\prime} P_{a}^{\prime \prime} ; r \geq \max _{\langle\mathcal{P}, R\rangle} \max \left\{\rho_{w} f_{2}\left(\Omega_{w}, P, R\right), f_{4}\left(\Omega_{w}^{\prime}\right)\right\},
$$

where $P_{a}$ is a primitive word, and $\langle\mathcal{P}, R\rangle$ runs through all the periodic structures of $\tilde{\Omega}_{w}$ such that either one of them is singular or for a solution with maximal number of short variables with respect to the group of extended automorphisms all the closed sections are potentially transferable. The proof of Proposition [7.5 will be completed after we prove the following statement.

Lemma 7.6. If $t p(w)=2$ and every closed section belonging to a periodic structure $\mathcal{P}$ is potentially transferable (the definition is given in the construction of $T_{0}$ in case 15), one can apply the second minimal replacement and get a finite number (depending on periodic structures containing this section in the vertices of type 2 in the trees $\left.T_{0}\left(w_{i}\right), i=1, \ldots, m\right)$ of possible generalized equations containing the same closed sections not from $\mathcal{P}$ and not containing closed sections from $\mathcal{P}$. 
Proof. From the definition of a potentially transferable section it follows that after finite number of transformations depending on $f_{4}\left(\Omega_{u}^{\prime}, \mathcal{P}\right)$, where $u$ runs through the vertices of type 2 in the trees $T_{0}\left(w_{i}\right), i=1, \ldots, m$, we obtain a cycle that is shorter than or equal to $d\left(\lambda_{\max }\right)$. This cycle is exactly $h\left[\alpha\left(\mu_{i}\right), \alpha\left(\Delta\left(\mu_{i}\right)\right]\right.$ for the base $\mu_{i}$ in the $\mu$-reducing subpath. The rest of the proof of Lemma 7.6 is a repetition of the proof of Lemma 6.18

7.5. Construction of the decomposition tree $T_{\operatorname{dec}}(\Omega)$ from $T_{0}(\Omega)$. We can define now a decomposition tree $T_{\operatorname{dec}}(\Omega)$. To obtain $T_{\operatorname{dec}}(\Omega)$ we add some edges to the terminal vertices of type 2 of $T_{0}(\Omega)$. Let $v$ be a vertex of type 2 in $T_{0}(\Omega)$. If there is no periodic structures in $\Omega_{v}$ then this is a terminal vertex of $T_{\operatorname{dec}}(\Omega)$. Suppose there exists a finite number of combinations of different periodic structures $\mathcal{P}_{1}, \ldots, \mathcal{P}_{s}$ in $\Omega_{v}$. If some $\mathcal{P}_{i}$ is singular, we consider a generalized equation $\Omega_{u\left(\mathcal{P}_{1}, \ldots, \mathcal{P}_{s}\right)}$ obtained from $\Omega_{v}\left(\mathcal{P}_{1}, \ldots, \mathcal{P}_{s}\right)$ by the first minimal replacement corresponding to $\mathcal{P}_{i}$. We also draw the edge $v \rightarrow u=u\left(\mathcal{P}_{1}, \ldots, \mathcal{P}_{s}\right)$. This vertex $u$ is a terminal vertex of $T_{\text {dec }}(\Omega)$. If all $\mathcal{P}_{1}, \ldots, \mathcal{P}_{s}$ in $\Omega_{v}$ are not singular, we can suppose that for each periodic structure $\mathcal{P}_{i}$ with period $P_{i}$ some values of variables in $\mathcal{P}_{i}$ are shorter than $2\left|P_{i}\right|$ and values of some other variables are shorter than $f_{3}\left(\Omega_{v}\right)\left|P_{i}\right|$, where $f_{3}$ is the function from Lemma 6.15 Then we apply the second minimal replacement. The resulting generalized equations $\Omega_{u_{1}}, \ldots, \Omega_{u_{t}}$ will have empty non-parametric part. We draw the edges $v \rightarrow u_{1}, \ldots, v \rightarrow u_{t}$ in $T_{\operatorname{dec}}(\Omega)$. Vertices $u_{1}, \ldots, u_{t}$ are terminal vertices of $T_{\operatorname{dec}}(\Omega)$.

Combining this construction with Theorem 7.1 and Proposition 7.5 we obtain the following theorem.

TheOREM 7.7. Let $\Omega$ be a generalized equation without parameters. To each branch of $T_{\mathrm{dec}}(\Omega)$ with terminal vertex $w$ one can assign a splitting (maybe degenerate) of $F_{R(\Omega)}$ such that every solution $H$ of $\Omega$ corresponding to this branch can be transformed by a canonical automorphism corresponding to this splitting into a solution $H^{+}$of $\Omega_{w}$. The group $F_{R\left(\Omega_{w}\right)}$ is a proper quotient of $F_{R(\Omega)}$.

\section{Structure of solutions, the solution tree $T_{\mathrm{sol}}(\Omega, \Lambda)$}

Let $\Omega=\Omega(H)$ be a generalized equation in variables $H$ with the set of bases $B_{\Omega}=B \cup \Lambda$. Let $T_{\mathrm{dec}}(\Omega)$ be the tree constructed in Subsection 7.5 for a generalized equation $\Omega$ with parameters $\Lambda$.

Recall that in a leaf-vertex $v$ of $T_{\mathrm{dec}}(\Omega)$ we have the coordinate group $F_{R\left(\Omega_{v}\right)}$ which is a proper homomorphic image of $F_{R(\Omega)}$. We define a new transformation $R_{v}$ (we call it leaf-extension) of the tree $T_{\operatorname{dec}}(\Omega)$ at the leaf vertex $v$. We take the union of two trees $T_{\mathrm{dec}}(\Omega)$ and $T_{\mathrm{dec}}\left(\Omega_{v}\right)$ and identify the vertices $v$ in both trees (i.e., we extend the tree $T_{\mathrm{dec}}(\Omega)$ by gluing the tree $T_{\mathrm{dec}}\left(\Omega_{v}\right)$ to the vertex $\left.v\right)$. Observe that if the equation $\Omega_{v}$ has non-parametric non-constant sections (in this event we call $v$ a terminal vertex), then $T_{\mathrm{dec}}\left(\Omega_{v}\right)$ consists of a single vertex, namely $v$.

Now we construct a solution tree $T_{\text {sol }}(\Omega)$ by induction starting at $T_{\mathrm{dec}}(\Omega)$. Let $v$ be a leaf non-terminal vertex of $T^{(0)}=T_{\mathrm{dec}}(\Omega)$. Then we apply the transformation $R_{v}$ and obtain a new tree $T^{(1)}=R_{v}\left(T_{\mathrm{dec}}(\Omega)\right)$. If there exists a leaf non-terminal

vertex $v_{1}$ of $T^{(1)}$, then we apply the transformation $R_{v_{1}}$, and so on. By induction we construct a strictly increasing sequence of trees

$$
T^{(0)} \subset T^{(1)} \subset \ldots \subset T^{(i)} \subset \ldots
$$


This sequence is finite. Indeed, suppose to the contrary that the sequence is infinite and hence the union $T^{(\infty)}$ of this sequence is an infinite tree in which every vertex has a finite degree. By Konig's lemma there is an infinite branch $B$ in $T^{(\infty)}$. Observe that along any infinite branch in $T^{(\infty)}$ one has to encounter infinitely many proper epimorphisms. This contradicts the fact that $F$ is equationally Noetherian.

Denote the union of the sequence of the trees (70) by $T_{\text {sol }}(\Omega, \Lambda)$. We call $T_{\text {sol }}(\Omega, \Lambda)$ the solution tree of $\Omega$ with parameters $\Lambda$. Recall that with every edge $e$ in $T_{\mathrm{dec}}(\Omega)$ (as well as in $T_{\mathrm{sol}}(\Omega, \Lambda)$ ) with the initial vertex $v$ and the terminal vertex $w$ we associate an epimorphism

$$
\pi_{e}: F_{R\left(\Omega_{v}\right)} \rightarrow F_{R\left(\Omega_{v}\right)} .
$$

It follows that every connected (directed) path $p$ in the graph gives rise to a composition of homomorphisms which we denote by $\pi_{p}$. Since $T_{\text {sol }}(\Omega, \Lambda)$ is a tree the path $p$ is completely defined by its initial and terminal vertices $u, v$; in this case we sometimes write $\pi_{u, v}$ instead of $\pi_{p}$. Let $\pi_{v}$ be the homomorphism corresponding to the path from the initial vertex $v_{0}$ to a given vertex $v$, we call it the canonical epimorphism from $F_{R(\Omega)}$ onto $F_{R\left(\Omega_{v}\right)}$.

Also, with some vertices $v$ in the tree $T_{\mathrm{dec}}(\Omega)$, as well as in the tree $T_{\mathrm{sol}}(\Omega, \Lambda)$, we associate groups of canonical automorphisms $A\left(\Omega_{v}\right)$ or extended automorphisms $\bar{A}\left(\Omega_{v}\right)$ of the coordinate group $F_{R\left(\Omega_{v}\right)}$ which, in particular, fix all variables in the non-active part of $\Omega_{v}$. We can suppose that the group $\bar{A}\left(\Omega_{v}\right)$ is associated to every vertex, but for some vertices it is trivial. Observe also, that canonical epimorphisms map parametric parts into parametric parts (i.e., subgroups generated by variables in parametric parts).

Recall that writing $(\Omega, U)$ means that $U$ is a solution of $\Omega$. If $(\Omega, U)$ and $\mu \in B_{\Omega}$, then by $\mu_{U}$ we denote the element

$$
\mu_{U}=\left[u_{\alpha(\mu)} \ldots u_{\beta(\mu)-1}\right]^{\varepsilon(\mu)} .
$$

Let $B_{U}=\left\{\mu_{U} \mid \mu \in B\right\}$ and $\Lambda_{U}=\left\{\mu_{U} \mid \mu \in \Lambda\right\}$. We refer to these sets as the set of values of bases from $B$ and the set of values of parameters from $\Lambda$ with respect to the solution $U$. Notice, that the value $\mu_{U}$ is given in (71) as a value of one fixed word mapping

$$
P_{\mu}(H)=\left[h_{\alpha(\mu)} \ldots h_{\beta(\mu)-1}\right]^{\varepsilon(\mu)} .
$$

In vector notation we can write that

$$
B_{U}=P_{B}(U), \quad \Lambda_{U}=P_{\Lambda}(U),
$$

where $P_{B}(H)$ and $P_{\Lambda}(H)$ are corresponding word mappings.

The following result explains the name of the tree $T_{\text {sol }}(\Omega, \Lambda)$.

THEOREM 8.1. Let $\Omega=\Omega(H, \Lambda)$ be a generalized equation in variables $H$ with parameters $\Lambda$. Let $T_{\mathrm{sol}}(\Omega, \Lambda)$ be the solution tree for $\Omega$ with parameters. Then the following conditions hold.

(1) For any solution $U$ in $F$ of the generalized equation $\Omega$ there exist: a path $v_{0} \rightarrow v_{1} \rightarrow \ldots \rightarrow v_{n}=v$ in $T_{\text {sol }}(\Omega, \Lambda)$ from the root vertex $v_{0}$ to a terminal vertex $v$, an abelian splitting of each of $F_{R\left(\Omega_{v_{0}}\right)}, \ldots, F_{R\left(\Omega_{v_{n}}\right)}$, a sequence of canonical automorphisms $\sigma=\left(\sigma_{0}, \ldots, \sigma_{n}\right), \sigma_{i} \in A\left(\Omega_{v_{i}}\right)$, and a solution $U_{v}$ in $F$ of the generalized equation $\Omega_{v}$ such that the solution $U$ (viewed 
as a homomorphism $\left.F_{R(\Omega)} \rightarrow F\right)$ is equal to the following composition of homomorphisms

$$
U=\Phi_{\sigma, U_{v}}=\sigma_{0} \pi_{v_{0}, v_{1}} \sigma_{1} \ldots \pi_{v_{n-1}, v_{n}} \sigma_{n} U_{v} .
$$

(2) For any path $v_{0} \rightarrow v_{1} \rightarrow \ldots \rightarrow v_{n}=v$ in $T_{\text {sol }}(\Omega, \Lambda)$ from the root vertex $v_{0}$ to a terminal vertex $v$, a pair consisting of a sequence of canonical automorphisms $\sigma=\left(\sigma_{0}, \ldots, \sigma_{n}\right), \sigma_{i} \in A\left(\Omega_{v_{i}}\right)$, and a solution $U_{v}$ in $F$ of the generalized equation $\Omega_{v}$, the composition $\Phi_{\sigma, U_{v}}$, gives a solution in $F$ of the corresponding group equation $\Omega^{*}=1$; moreover, every solution in $F$ of $\Omega^{*}=1$ can be obtained this way.

(3) For each terminal vertex $v$ in $T_{\text {sol }}(\Omega, \Lambda)$ there exists a word mapping $Q_{v}\left(H_{v}\right)$ such that for any solution $U_{v}$ of $\Omega_{v}$ and any solution $U=\Phi_{\sigma, U_{v}}$ from (72) the values of the parameters $\Lambda$ with respect to $U$ can be written as $\Lambda_{U}=Q_{v}\left(U_{v}\right)$ (i.e., these values do not depend on $\sigma$ ) and the word $Q_{v}\left(U_{v}\right)$ is reduced as written.

Proof. Statements (1) and (2) follow from the construction of the tree $T_{\text {sol }}(\Omega, \Lambda)$. To verify (3) we need to invoke the argument above this theorem which claims that the canonical automorphisms associated with generalized equations in $T_{\text {sol }}(\Omega, \Lambda)$ fix all variables in the parametric part and, also, that the canonical epimorphisms map variables from the parametric part into themselves.

TheOREM 8.2. For any finite system $S(X)=1$ over a free group $F$, one can find effectively a finite family of nondegenerate triangular quasi-quadratic systems $U_{1}, \ldots, U_{k}$ and word mappings $p_{i}: V_{F}\left(U_{i}\right) \rightarrow V_{F}(S)(i=1, \ldots, k)$ such that for every $b \in V_{F}(S)$ there exists $i$ and $c \in V_{F}\left(U_{i}\right)$ for which $b=p_{i}(c)$, i.e.

$$
V_{F}(S)=p_{1}\left(V_{F}\left(U_{1}\right)\right) \cup \ldots \cup p_{k}\left(V_{F}\left(U_{k}\right)\right)
$$

and all sets $p_{i}\left(V_{F}\left(U_{i}\right)\right)$ are irreducible; moreover, every irreducible component of $V_{F}(S)$ can be obtained as a closure of some $p_{i}\left(V_{F}\left(U_{i}\right)\right)$ in the Zariski topology.

Proof. Each solution of the system $S(X)=1$ can be obtained as $X=p_{i}\left(Y_{i}\right)$, where $Y_{i}$ are variables of $\Omega=\Omega_{i}$ for a finite number of generalized equations. We have to show that all solutions of $\Omega^{*}$ are solutions of some NTQ system. We can use Theorem 8.1 without parameters and Theorem 7.1. In this case $\Omega_{v}$ is an empty equation with non-empty set of variables. In other words $F_{R\left(\Omega_{v}\right)}=$ $F * F\left(h_{1}, \ldots, h_{\rho}\right)$. To each of the branches of $T_{\text {sol }}$ we assign an NTQ system from the formulation of the theorem. Let $\Omega_{w}$ be a leaf vertex in $T_{\text {dec }}$. Then $F_{R\left(\Omega_{w}\right)}$ is a proper quotient of $F_{R(\Omega)}$. Consider the path $v_{0}, v_{1}, \ldots, v_{n}=w$ in $T_{\text {dec }}(\Omega)$ from the root vertex $v_{0}$ to a terminal vertex $w$. All the groups $F_{R\left(\Omega_{v_{i}}\right)}$ are isomorphic. There are the following five possibilities.

1. $t p\left(v_{n-1}\right)=2$. In this case there is a singular periodic structure on $\Omega_{v_{n-1}}$. By Lemma 6.7 $F_{R\left(\Omega_{v_{n-1}}\right)}$ is a fundamental group of a graph of groups with one vertex group $K$, some free abelian vertex groups, and some edges defining HNN extensions of $K$. Recall that making the first minimal replacement we first replaced $F_{R\left(\Omega_{v_{n-1}}\right)}$ by a finite number of proper quotients in which the edge groups corresponding to abelian vertex groups and HNN extensions are maximal cyclic in $K$. Extend the centralizers of the edge groups of $\Omega_{v_{n-1}}$ corresponding to HNN extensions by stable letters $t_{1}, \ldots, t_{k}$. This new group that we denote by $N$ is the coordinate group of a quadratic equation over $F_{R\left(\Omega_{w}\right)}$ which has a solution in $F_{R\left(\Omega_{w}\right)}$. 
In all the other cases $t p\left(v_{n-1}\right) \neq 2$.

2. There were no auxiliary edges from vertices $v_{0}, v_{1}, \ldots, v_{n}=w$, and if one of the Cases 7-10 appeared at one of these vertices, then it only appeared a bounded (the boundary depends on $\Omega_{v_{0}}$ ) number of times in the sequence. In this case we replace $F_{R(\Omega)}$ by $F_{R\left(\Omega_{w}\right)}$

3. $F_{R\left(\Omega_{w}\right)}$ is a term in a free decomposition of $F_{R\left(\Omega_{v_{n-1}}\right)}$ ( $\Omega_{w}$ is a kernel of a generalized equation $\left.\Omega_{v_{n-1}}\right)$. In this case we also consider $F_{R\left(\Omega_{w}\right)}$ instead of $F_{R(\Omega)}$.

4. For some $i t p\left(v_{i}\right)=12$ and the path $v_{i}, \ldots, v_{n}=w$ does not contain vertices of type $7-10,12$ or 15 . In this case $F_{R(\Omega)}$ is the coordinate group of a quadratic equation.

5. The path $v_{0}, v_{1}, \ldots, v_{n}=w$ contains vertices of type 15 . Suppose $v_{i_{j}}, \ldots, v_{i_{j}+k_{j}}$, $j=1, \ldots, l$ are all blocks of consecutive vertices of type 15 This means that $t p\left(v_{i_{j}+k_{j}+1}\right) \neq 15$ and $i_{j}+k_{j}+1<i_{j+1}$. Suppose also that none of the previous cases takes place. To each $v_{i_{j}}$ we assigned a quadratic equation and a group of canonical automorphisms corresponding to this equation. Going along the path $v_{i_{j}}, \ldots, v_{i_{j}+k_{j}}$, we take minimal solutions corresponding to some non-singular periodic structures. Each such structure corresponds to a representation of $F_{R\left(\Omega_{v_{i_{j}}}\right)}$ as an HNN extension. As in the case of a singular periodic structure, we can suppose that the edge groups corresponding to HNN extensions are maximal cyclic and not conjugated in $K$. Extend the centralizers of the edge groups corresponding to HNN extensions by stable letters $t_{1}, \ldots, t_{k}$. Let $N$ be the new group. Then $N$ is the coordinate group of a quadratic system of equations over $F_{R\left(\Omega_{v_{i_{j}+k_{j}+1}}\right)}$. Repeating this construction for each $j=1, \ldots, l$, we construct NTQ system over $F_{R\left(\Omega_{w}\right)}$.

Since $F_{R\left(\Omega_{w}\right)}$ is a proper quotient of $F_{R(\Omega)}$, the theorem can now be proved by induction.

\section{Maximal standard quotients and canonical embeddings of $\mathcal{F}$-groups}

In this section we construct standard quotients and canonical embeddings of freely indecomposable finitely generated fully residually free groups into NTQ groups.

9.1. JSJ-decompositions are sufficient splittings. In this section we prove that a non-degenerate JSJ decomposition $D$ of a finitely generated freely indecomposable fully residually free group $G$ is a sufficient splitting of $G$, i.e., the standard maximal quotient $G / R_{D}$ is a proper quotient. Let, as usual, $F$ be a fixed free non-abelian group.

TheOREM 9.1. Let $G$ be a finitely generated freely indecomposable [freely indecomposable modulo $F]$ fully residually free group $G$. Then a non-degenerate JSJ $\mathbb{Z}$ decomposition [modulo $F$ ] of $G$ is a sufficient splitting of $G$.

Proof. Let $D$ be a non-degenerate $J S J$ decomposition of the group $G=$ $F_{R(U)}$. Recall, that by $\mathcal{G} E(U)$ we denote the finite set of the initial generalized equations associated with $U=1$ (see Section 4.2). For each $\Omega \in \mathcal{G} E(U)$ we are going to construct a tree $T_{m q}(\Omega)$ as follows. Let $T_{\operatorname{dec}}(\Omega)$ be the decomposition tree which has been constructed in Subsection 7.5. Suppose $v$ is a leaf vertex of $T_{\operatorname{dec}}(\Omega)$ and $\Omega_{v}$ is the generalized equation associated with $v$ in $T_{\operatorname{dec}}(\Omega)$. By the construction the group $F_{R\left(\Omega_{v}\right)}$ is a proper quotient of $F_{R(\Omega)}$. If the image of $G$ in 
$F_{R\left(\Omega_{v}\right)}$ under the quotient epimorphism $F_{R(\Omega)} \rightarrow F_{R\left(\Omega_{v}\right)}$ is not a proper quotient of $G$, then we extend the tree $T_{\operatorname{dec}}(\Omega)$ by gluing up the tree $T_{\operatorname{dec}}\left(\Omega_{v}\right)$ to the vertex $v$ (by identifying the vertex $v$ in $T_{\mathrm{dec}}(\Omega)$ with the root vertex in $T_{\mathrm{dec}}\left(\Omega_{v}\right)$ ). Denote the resulting tree by $T_{\mathrm{dec}}(\Omega)^{\prime}$. Again, if there is a leaf vertex $v^{\prime} \in T_{\operatorname{dec}}(\Omega)^{\prime}$ such that the canonical image of $G$ in the group associated with $v^{\prime}$ is not a proper quotient of $G$ then we extend $T_{\operatorname{dec}}(\Omega)^{\prime}$ by gluing up the tree $T_{\operatorname{dec}}\left(\Omega_{v}^{\prime}\right)$ to the vertex $v^{\prime}$ (we refer to this operation as to leaf extension). More formally, let $T_{0}=T_{\mathrm{dec}}(\Omega)$. Suppose a tree $T_{i}$ with associated groups and homomorphisms is already constructed. If there is a vertex $w \in T_{i}$ such that the image of $G$ in the group associated with $w$ is not a proper quotient of $G$ then we glue up the tree $T_{\operatorname{dec}}\left(\Omega_{w}\right)$ to the vertex $w$ (by identifying $w$ with the root vertex in $T_{\operatorname{dec}}\left(\Omega_{w}\right)$ ) and denote the resulting tree by $T_{i+1}$.

\section{Claim. The sequence}

$$
T_{0} \subset T_{1} \subset T_{2} \subset \ldots
$$

is finite.

Indeed, suppose the sequence (73) is infinite. Then the graph $T=\cup_{i=1}^{\infty} T_{i}$ is infinite. Then by Konig's lemma there is an infinite branch in $T$, say,

$$
v_{0} \rightarrow v_{1} \rightarrow v_{2} \rightarrow \ldots
$$

Therefore there exists an infinite subsequence, say,

$$
v_{i_{0}} \rightarrow v_{i_{1}} \rightarrow v_{i_{2}} \rightarrow \ldots
$$

such that for every vertex $v_{i_{j}}$ in this sequence at some step the tree $T_{\mathrm{dec}}\left(\Omega_{v_{i_{j}}}\right)$ was glued to the vertex $v_{i_{j}}$. It follows that the homomorphism corresponding to the edge $v_{i_{j}-1} \rightarrow v_{i_{j}}$ is a proper epimorphism. Hence there are infinitely many proper epimorphisms associated with the edges of the branch (74). This implies that there exists an infinite sequence of epimorphisms of finitely generated residually free groups which contains infinitely many proper epimorphisms - contradiction with the equationally Noetherian property of free groups. This proves the claim.

Denote the union tree $T$ of the finite sequence (73) by $T_{m q}(\Omega)$. Fix some terminal vertex $w$ of $T_{m q}(U, \Omega)$. Each homomorphism from the image of $G$ in $F_{R\left(\Omega_{w}\right)}$ into $F$ factors through a finite number of fully residually free quotients. Denote one such quotient by $\bar{G}=F_{R(\bar{U})} * F\left(t_{1}, \ldots, t_{k}\right)$, where $\bar{U}$ is irreducible, and $t_{1}, \ldots, t_{k}$ are free variables from quadratic equations.

Let $v_{0}$ be the initial vertex of $T_{\operatorname{dec}}(\Omega), v_{1}$ be the terminal vertex of $T_{\operatorname{dec}}(\Omega)$, $v_{2}$ be the terminal vertex of $T_{\operatorname{dec}}\left(\Omega_{v_{1}}\right)$ and so on, $v_{n}=w$. Each solution of $U=1$ corresponding to this branch of $T_{m q}(U, \Omega)$ can be transformed by canonical automorphisms corresponding to vertices of $T_{\mathrm{dec}}(\Omega)$ (which are canonical automorphisms from $A_{D_{0}}$, where $D_{0}$ is a decomposition of $F_{R(\Omega)}$, see Subsection 7.2) and, subsequently, by canonical automorphisms corresponding to vertices of $T_{\mathrm{dec}}\left(\Omega_{v_{i}}\right), i=1, \ldots, n$ (which are canonical automorphisms from $A_{D_{i}}$, where $D_{i}$ is a decomposition of $\left.F_{R\left(\Omega_{v_{i}}\right)}\right)$, into a solution satisfying a proper equation.

By Lemma 2.13, for each QH subgroup $Q$ in $F_{R\left(\Omega_{v_{i}}\right)}$, either the intersection of $G$ with some conjugate $Q^{g}$ is of finite index in $Q^{g}$ and is a $\mathrm{QH}$ subgroup of $G$, or $G$ is conjugated into the fundamental group of a connected component of the graph of groups obtained from $D$ by removing the vertex and edges corresponding to $Q$. Case 1) in the lemma cannot take place because $G$ is freely indecomposable. In 
the case when $G$ is conjugated into the fundamental group of the graph of groups obtained from $D$ by removing the vertex and edges corresponding to $Q$, each solution of $U=1$ can be obtained from a solution of $\Omega_{v_{i}}$ minimal with respect to the canonical group of automorphisms corresponding to $Q$. If $G$ has trivial intersection with all conjugates of some edge group of $D_{i}$, then again each solution of $U=1$ can be obtained from a solution of $\Omega_{v_{i}}$ minimal with respect to the group generated by the canonical Dehn twists along this edge. If the intersection $G \cap Q^{g}$ has a finite index, then it is a $Q H$ subgroup $Q_{1}$ of $G$ and the group of automorphisms of $Q_{1}$ induced by canonical automorphisms of $Q^{g}$ has finite index in the group of canonical automorphisms of $Q^{g}$. Let $\bar{F}_{R\left(\Omega_{v_{i}}\right)}$ be the factor that contains $G$ in some Grushko decomposition of $F_{R\left(\Omega_{v_{i}}\right)}$. Let $A\left(\bar{F}_{R\left(\Omega_{v_{i}}\right)}\right)$ be the group of outer canonical automorphisms of $\bar{F}_{R\left(\Omega_{v_{i}}\right)}$ induced from canonical automorphisms of $F_{R\left(\Omega_{v_{i}}\right)}$. By Lemma 2.13 the group of canonical automorphisms of $\bar{F}_{R\left(\Omega_{v_{i}}\right)}$ corresponding to $Q^{g}$ (where $Q$ is a QH subgroup of $\left.\bar{F}_{R\left(\Omega_{v_{i}}\right)}\right)$ that induce canonical automorphisms of $G$ has finite index in the subgroup of $A\left(\bar{F}_{R\left(\Omega_{v_{i}}\right)}\right)$ corresponding to $Q$. Denote by $A_{G}\left(\bar{F}_{R\left(\Omega_{v_{i}}\right)}\right)$ the subgroup of $A\left(\bar{F}_{R\left(\Omega_{v_{i}}\right)}\right)$ generated by canonical Dehn twists corresponding to the splittings of $\bar{F}_{R\left(\Omega_{v_{i}}\right)}$ that induce non-trivial splittings of $G$. The subgroup $A_{\text {ind }}$ of $A_{G}\left(\bar{F}_{R\left(\Omega_{v_{i}}\right)}\right)$ consisting of automorphisms that induce automorphisms of $G$, has finite index in $A_{G}\left(\bar{F}_{R\left(\Omega_{v_{i}}\right)}\right)$.

This implies that using canonical automorphisms corresponding to the JSJ decomposition of $G$, we can transform each solution $\phi$ of $U=1$ into a solution that is not longer than $\phi$ and satisfies one of the finite number of proper equations (which can be effectively found) or, equivalently, satisfies a proper equation which represents this disjunction of equations. Since a minimal solution in the equivalence class of $\phi$ factors through some branch of $T_{m q}(\Omega)$ for one of the finite number of generalized equations $\Omega_{w}$, it must satisfy this proper equation.

9.2. Quasi-quadratic systems associated with $\mathbb{Z}$-splittings. Let $G$ be a finitely generated freely indecomposable fully residually free group and $D$ a nondegenerate JSJ $\mathbb{Z}$-decomposition of $G$. Combining foldings and slidings, we can transform $D$ into an abelian decomposition in which each vertex with non-cyclic abelian subgroup that is connected to some rigid vertex, is connected to only one vertex which is rigid. We suppose from the beginning that $D$ has this property. Let $L_{i}, i \in I$ be a (finite) collection of all standard maximal fully residually free quotients of $G$ relative to $D$ and $\pi_{i}: G \rightarrow L_{i}$ the corresponding canonical epimorphisms. By Corollary 2.28 one can enumerate these quotients as

$$
L_{1}, \ldots, L_{k}, \ldots, L_{k+s}, \quad k \geq 1, s \geq 0,
$$

in such a way that for each $i=1, \ldots, k$ all the restrictions of $\pi_{i}$ onto rigid subgroups of $D$, onto edge subgroups in $D$, and onto the subgroups of the abelian vertex groups $A$ generated by the images of all the edge groups of edges adjacent to $A$, are monomorphisms. Let $H_{1}, \ldots, H_{q}$ be rigid subgroups of $G$ in $D$. Consider the Grushko's decomposition of $L_{i}$ compatible with the collection of subgroups $\pi\left(H_{1}\right), \ldots, \pi\left(H_{q}\right)$ :

$$
L_{i}=L_{i, 1} * \ldots * L_{i, \alpha_{i}} * F\left(t_{1}, \ldots, t_{\beta_{i}}\right),
$$


where $F\left(t_{1}, \ldots, t_{\beta_{i}}\right)$ is the maximal free factor that does not contain conjugates of any of the subgroups $\pi\left(H_{1}\right), \ldots, \pi\left(H_{q}\right)$. Denote

$$
\bar{G}_{i}=L_{i, 1} * \ldots * L_{i, \alpha_{i}} .
$$

In this section we show that $G$ embeds into a group $G_{i}$ which is a particular extension of the group $\bar{G}_{i}, i=1, \ldots, k$. More precisely, $G_{i}$ is the fundamental group of a graph of groups $\Gamma_{i}$ obtained from a single vertex $v$ with the associated vertex group $G_{v}=\bar{G}_{i}$ by adding finitely many edges corresponding to extensions of centralizers (viewed as amalgamated products) and finitely many $\mathrm{QH}$ vertices connected only to $v$ (see Figure 15).

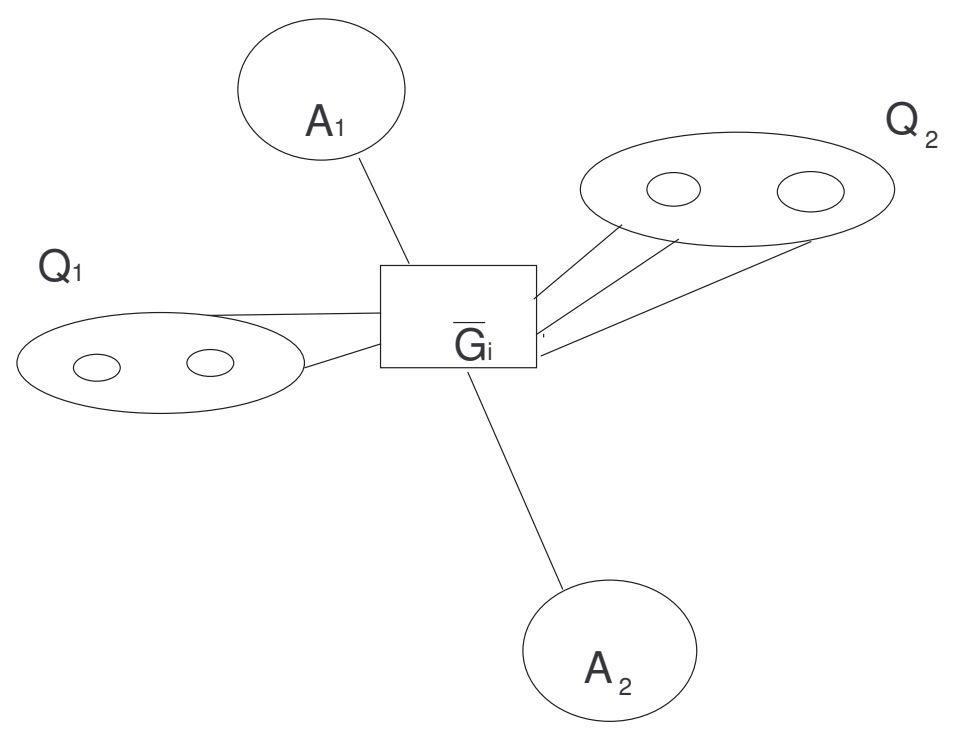

Figure 15. The graph of groups $\Gamma_{i}$ of the coordinate group of the quasi-quadratic system over $\bar{G}_{i}$.

We start with description of the extensions $G_{i}$ of $\bar{G}$. Let $g_{1}, \ldots, g_{l}$ be a fixed finite generating set of $\bar{G}$. For an edge $e \in D$ we fix a generator $d_{e}$ of the cyclic edge group $G_{e}$ (or generators of an abelian edge group connecting a non-cyclic abelian vertex group to a rigid subgroup). Fix an integer $i, 1 \leq i \leq k$ and, to simplify notation, denote $\bar{G}_{i}$ by $\bar{G}, \pi_{i}$ by $\pi$, and $G_{i}$ by $G^{*}$. The required extension $G^{*}$ of $\bar{G}$ is constructed in three steps. On each step we extend the centralizers $C_{\bar{G}}\left(\pi\left(d_{e}\right)\right)$ of some edges $e$ in $D$ or add a QH subgroup. Simultaneously, for every edge $e \in D$ we associate an element $s_{e} \in C_{G^{*}}\left(\pi\left(d_{e}\right)\right)$.

Step 1. Let $E_{\text {rig }}$ be the set of all edges between rigid subgroups in $D$. For each edge $e \in E_{\text {rig }}$ we do the following. If

$$
G=A *_{\left\langle d_{e}\right\rangle} B \text { and } \bar{G}=\pi(A) *\left\langle\pi\left(d_{e}\right)\right\rangle \pi(B)
$$

or

$$
G=A *\left\langle d_{e}\right\rangle \text { and } \bar{G}=\pi(A) *\left\langle\pi\left(d_{e}\right)\right\rangle
$$

then we delete the edge $e$ from $E_{\text {rig }}$. In this case we associate the trivial element $s_{e}=1$ with $e$. Denote the resulting set of edges by $E^{\prime}$. One can define an 
equivalence relation $\sim$ on $E^{\prime}$ assuming for $e, f \in E^{\prime}$ that

$$
e \sim f \Longleftrightarrow \exists g_{e f} \in \bar{G}\left(g_{e f}^{-1} C_{\bar{G}}(\pi(e)) g_{e f}=C_{\bar{G}}(\pi(e))\right) .
$$

Let $E$ be a set of representatives of equivalence classes of $E^{\prime}$ modulo $\sim$.

Now we construct a group $G^{(1)}$ by extending every centralizer $C_{\bar{G}}\left(\pi\left(d_{e}\right)\right)$ of $\bar{G}$, $e \in E$ as follows. Let

$$
[e]=\left\{e=e_{1}, \ldots, e_{q_{e}}\right\}
$$

and $y_{e}^{(1)}, \ldots, y_{e}^{\left(q_{e}\right)}$ be new letters corresponding to the elements in $[e]$. Then put $G^{(1)}=\left\langle\bar{G}, y_{e}^{(1)}, \ldots, y_{e}^{\left(q_{e}\right)}(e \in E) \mid\left[C\left(\pi\left(d_{e}\right)\right), y_{e}^{(j)}\right]=1,\left[y_{e}^{(i)}, y_{e}^{(j)}\right]=1\left(i, j=1, \ldots, q_{e}\right)\right\rangle$.

One can associate with $G^{(1)}$ the following system of equations over $\bar{G}$ :

$$
\left[\bar{g}_{e s}, y_{e}^{(j)}\right]=1,\left[y_{e}^{(i)}, y_{e}^{(j)}\right]=1, \quad i, j=1, \ldots, q_{e}, s=1, \ldots, p_{e}, e \in E,
$$

where $y_{e}^{(j)}$ are new variables and the elements $\bar{g}_{e 1}, \ldots, \bar{g}_{e p_{e}}$ are constants from $\bar{G}$ which generate the centralizer $C\left(\pi\left(d_{e}\right)\right)$. We assume that the constants $\bar{g}_{e j}$ are given as words in the generators $g_{1}, \ldots, g_{l}$ of $\bar{G}$. We associate the element $s_{e_{i}}=y_{e}^{(i)}$ with the edge $e=e_{i}$.

Step 2. Let $A$ be a non-cyclic abelian vertex group in $D$ and $A_{e}$ the subgroup of $A$ generated by the images in $A$ of the edge groups of edges adjacent to $A$. Then $A=I s\left(A_{e}\right) \times A_{0}$ where $I s\left(A_{e}\right)$ is the isolator of $A_{e}$ in $A$ (the minimal direct factor containing $\left.A_{e}\right)$ and $A_{0}$ a direct complement of $I s\left(A_{e}\right)$ in $A$. Notice, that the restriction of $\pi$ on $I s\left(A_{e}\right)$ is a monomorphism (since $\pi$ is injective on $A_{e}$ and $A_{e}$ is of finite index in $\left.I s\left(A_{e}\right)\right)$. For each non-cyclic abelian vertex group $A$ in $D$ we extend the centralizer of $\pi\left(I s\left(A_{e}\right)\right)$ in $G^{(1)}$ by the abelian group $A_{0}$ and denote the resulting group by $G^{(2)}$. Observe, that since $\pi\left(I s\left(A_{e}\right)\right) \leq \bar{G}$ the group $G^{(2)}$ is obtained from $\bar{G}$ by extending finitely many centralizers of elements from $\bar{G}$.

If the abelian group $A_{0}$ has rank $r$ then the system of equations associated with the abelian vertex group $A$ has the following form

$$
\left[y_{p}, y_{q}\right]=1,\left[y_{p}, \bar{d}_{e j}\right]=1, \quad p, q=1, \ldots, r, j=1, \ldots, p_{e},
$$

where $y_{p}, y_{q}$ are new variables and the elements $\bar{d}_{e 1}, \ldots, \bar{d}_{e p_{e}}$ are constants from $\bar{G}$ which generate the subgroup $\pi\left(I s\left(A_{e}\right)\right)$. We assume that the constants $\bar{d}_{e j}$ are given as words in the generators $g_{1}, \ldots, g_{l}$ of $\bar{G}$.

Denote by

$$
\bar{S}_{i}\left(y_{1}, \ldots, y_{t}, g_{1}, \ldots, g_{l}\right)=1
$$

the union of the system (75) and all the systems (76) for every abelian non-cyclic vertex group $A$ in $D$. Here $y_{1}, \ldots, y_{t}$ are all the new variables that occur in the equations (75), (76).

Step 3. A QH subgroup $Q$ such that $\pi(Q)$ is a $\mathrm{QH}$ subgroup of $\bar{G}$ of the same size (see Section 1.6) is called a stable $Q H$ subgroup. Let $Q$ be a non-stable $\mathrm{QH}$ subgroup in $D$. Suppose $Q$ is given by a presentation

$$
\prod_{i=1}^{n}\left[x_{i}, y_{i}\right] p_{1} \ldots p_{m}=1
$$


where there are exactly $m$ outgoing edges $e_{1}, \ldots, e_{m}$ from $Q$ and $\sigma\left(G_{e_{i}}\right)=\left\langle p_{i}\right\rangle$, $\tau\left(G_{e_{i}}\right)=\left\langle c_{i}\right\rangle$ for each edge $e_{i}$. We add a $\mathrm{QH}$ vertex $Q$ to $G^{(2)}$ by introducing new generators and the following quadratic relation

$$
\prod_{i=1}^{n}\left[x_{i}, y_{i}\right]\left(c_{1}^{\pi_{i}}\right)^{z_{1}} \ldots\left(c_{m-1}^{\pi_{i}}\right)^{z_{m-1}} c_{m}^{\pi_{i}}=1
$$

to the presentation of $G^{(2)}$. Observe, that in the relations (77) the coefficients in the original quadratic relations for $Q$ in $D$ are replaced by their images in $\bar{G}$.

Similarly, one introduces QH vertices for non-orientable QH subgroups in $D$. We refer to Section 2.9 for details on quadratic relations associated with nonorientable QH subgroups.

The resulting group is denoted by $G^{*}=G^{(3)}$.

Let

$$
S=S\left(z_{1}, \ldots, z_{m}, g_{1}, \ldots, g_{l}\right)=1
$$

be union of the quadratic systems of the type (77) for orientable and non-orientable non-stable QH subgroups in $D$. Here the set of variables $\left\{z_{1}, \ldots, z_{m}\right\}$ is union of all variables in quadratic equations of the type (77). Let $D^{*}$ be a splitting of $G^{*}$ with the following vertex groups: factors $L_{1}, \ldots, L_{\alpha}$ in the compatible reduced free decomposition of $\bar{G}$, abelian vertex groups corresponding to relations (75), (76), and QH subgroups added to $\bar{G}$. Let $T^{*}$ be a maximal subtree in the underlying graph $\Gamma^{*}$. So far we defined elements $s_{e}$ only for edges between two rigid subgroups of $D$.

Now we define an $F$-homomorphism $\psi: G \rightarrow G^{*}$ and show that $\psi$ is injective. In the process we will associate elements $s_{e}$ to the remaining edges of $D$. We need the following lemma.

Lemma 9.2. (1) Let $H=A *\langle d\rangle B$ and $\pi: H \rightarrow \bar{H}$ be a homomorphism such that the restrictions of $\pi$ on $A$ and $B$ are injective. Put

$$
H^{*}=\left\langle\bar{H}, y \mid\left[C_{\bar{H}}(\pi(d)), y\right]=1\right\rangle .
$$

Then for every $u \in C_{H^{*}}\left((\pi(d)), u \notin C_{\bar{H}}(\pi(d))\right.$, a map

$$
\psi(x)= \begin{cases}\pi(x), & x \in A, \\ \pi(x)^{u}, & x \in B .\end{cases}
$$

gives rise to a monomorphism $\psi: H \rightarrow H^{*}$.

(2) Let $H=\left\langle A, t \mid d^{t}=c\right\rangle$ and $\pi: H \rightarrow \bar{H}$ be a homomorphism such that the restriction of $\pi$ on $A$ is injective. Put

$$
H^{*}=\left\langle\bar{H}, y \mid\left[C_{\bar{H}}(\pi(d)), y\right]=1\right\rangle .
$$

Then for every $u \in C_{H^{*}}\left((\pi(d)), u \notin C_{\bar{H}}(\pi(d))\right.$, a map

$$
\psi(x)= \begin{cases}\pi(x), & x \in A, \\ u \pi(x), & x=t .\end{cases}
$$

gives rise to a monomorphism $\psi: H \rightarrow H^{*}$.

Proof. We prove (1), a similar argument proves (2). Clearly, the map $\psi$ agrees on the intersection $A \cap B$, so it defines a homomorphism $\psi: H_{\text {rig }} \rightarrow G$. Straightforward verification shows that $\psi$ maps reduced forms of elements in $H_{\text {rig }}$ into reduced forms of elements in $G$. This proves that $\psi$ is injective. 
We define the homomorphism $\psi: G \rightarrow G^{*}$ with respect to the splitting $D$ of $G$. Let $T$ be the maximal subtree of $D$. First, we define $\psi$ on the fundamental group of the graph of groups induced from $D$ on $T$. Notice that the $\operatorname{subgroup} F$ is elliptic in $D$, so there is a rigid vertex $v_{0} \in T$ such that $F \leq G_{v_{0}}$. Mapping $\pi$ embeds $G_{v_{0}}$ into $\bar{G}$, hence into $G^{*}$.

Let $P$ be a path $v_{0} \rightarrow v_{1} \rightarrow \ldots \rightarrow v_{n}$ in $T$ that starts at $v_{0}$. With each edge $e_{i}=\left(v_{i-1} \rightarrow v_{i}\right)$ between two rigid vertex groups we have already associated the element $s_{e_{i}}$. Let us associate elements to other edges of $P$ :

a) if $v_{i-1}$ is a rigid vertex, and $v_{i}$ is either abelian or $\mathrm{QH}$, then $s_{e_{i}}=1$;

b) if $v_{i-1}$ is a QH vetrex, $v_{i}$ is rigid or abelian, and the image of $e_{i}$ in the decomposition $D^{*}$ of $G^{*}$ does not belong to $T^{*}$, then $s_{e_{i}}$ is the stable letter corresponding to the image of $e_{i}$;

c) if $v_{i-1}$ is a $\mathrm{QH}$ vertex and $v_{i}$ is rigid or abelian, and the image of $e_{i}$ in the decomposition of $G^{*}$ belongs to $T^{*}$, then $s_{e_{i}}=1$.

d) if $v_{i-1}$ is an abelian vertex with $G_{v_{i-1}}=A$ and $v_{i}$ is a $\mathrm{QH}$ vertex, then $s_{e_{i}}$ is an element from $A$ that belongs to $A_{0}$.

Since two abelian vertices cannot be connected by an edge in $\Gamma$, and we can suppose that two $\mathrm{QH}$ vertices are not connected by an edge, these are all possible cases.

We now define the embedding $\psi$ on the fundamental group corresponding to the path $P$ as follows:

$$
\psi(x)=\pi(x)^{s_{e_{i}} \ldots s_{e_{1}}} \text { for } x \in G_{v_{i}} .
$$

This map is a monomorphism by Lemma 9.2 Similarly we define $\psi$ on the fundamental group of the graph of groups induced from $D$ on $T$. We extend it to $G$ using the second statement of Lemma 9.2

We constructed a finite number of proper fully residually free quotients of $G$ : $\bar{G}, \ldots, \bar{G}_{k}$ where the systems $S=1 \wedge \bar{S}_{i}=1$ have solutions. Let $\bar{G}_{i}=F_{R\left(\bar{U}_{i}\right)}$. Now $U_{D, i}\left(z_{1}, \ldots, z_{m}, z_{m+1}, \ldots, z_{m+k_{i}}\right)=1$ is the system

$$
\begin{aligned}
S\left(z_{1}, \ldots, z_{m}, g_{1}, \ldots, g_{l}\right) & =1 \\
\bar{S}_{i}\left(y_{1}, \ldots, y_{t}, g_{1}, \ldots, g_{l}\right) & =1 \\
\bar{U}_{i}\left(g_{1}, \ldots, g_{l}\right) & =1
\end{aligned}
$$

If $G=F_{R(U)}$ is a surface group, we take the system $S=1$ the same as $U=1$, $\bar{S}_{i}=1$ and $\bar{U}_{i}=1$ trivial. If $G$ is a free abelian group, we take $\bar{S}=1$ the same as $U=1$, and $S=1, \bar{U}=1$ trivial. If $G$ is cyclic, we do not construct $U_{D, i}=1$.

We summarize the construction in the following theorem.

TheOREM 9.3. Let $U(X)=1, X=\left\{x_{1}, \ldots, x_{n}\right\}$, be a finite irreducible system of equations over $F$ such that $G=F_{R(U)}$ is freely indecomposable (modulo $F$ ) if $U(X)=1$ has non-trivial coefficients). Let $D$ be a JSJ $\mathbb{Z}$-decomposition of $G$. Then one can effectively construct finitely many equations

$$
V_{1}(X)=1, \ldots, V_{s}(X)=1
$$

with coefficients in $F$ and finitely many systems

$$
U_{D, 1}=1, \ldots, U_{D, k}=1
$$

over $F$ with embeddings

$$
\phi_{1}: F_{R(U)} \rightarrow F_{R\left(U_{D, 1}\right)}, \ldots, \phi_{k}: F_{R(U)} \rightarrow F_{R\left(U_{D, k}\right)}
$$


such that:

(1) $V_{i} \notin R(U), i=1, \ldots, s$.

(2) $U_{D, i}\left(z_{1}, \ldots, z_{m}, y_{1}, \ldots, y_{t}, z_{m+1}, z_{m+1}, \ldots, z_{m+k_{i}}\right)=1$ is a system of the following type

$$
\begin{aligned}
S\left(z_{1}, \ldots, z_{m}, y_{1}, \ldots, y_{t}, g_{1}, \ldots, g_{l}\right) & =1 \\
\bar{S}_{i}\left(y_{1}, \ldots, y_{t}, g_{1}, \ldots, g_{l}\right) & =1 \\
\bar{U}_{i}\left(g_{1}, \ldots, g_{l}\right) & =1
\end{aligned}
$$

where $\bar{U}_{i}=1$ is irreducible, $S=1$ is a non-degenerate quadratic system in variables $z_{1}, \ldots, z_{m}$ over $F_{R\left(\bar{U}_{i}\right)}$, variables $z_{1}, \ldots, z_{m}$ are standard generators of $M Q H$ subgroups of $G$ and stable letters corresponding to non-tree edges adjacent to $Q H$ subgroups, $\bar{S}_{i}=1$ corresponds to extensions of centralizers of elements from $F_{R\left(\bar{U}_{i}\right)}$.

(3) $F_{R\left(\bar{U}_{i}\right)}$ is a proper quotient of $F_{R(U)}$ with the canonical epimorphism $\eta_{i}$ : $F_{R(U)} \rightarrow F_{R\left(\bar{U}_{i}\right)}, i=1, \ldots, k$, which can be found effectively.

(4) $V(U)=p_{1}\left(V\left(U_{D, 1}\right)\right) \cup \ldots \cup p_{k}\left(V\left(U_{D, k}\right)\right) \cup V\left(U \wedge V_{1}\right) \cup \ldots \cup V\left(U \wedge V_{s}\right)$, where the word mappings $p_{i}=\left(p_{i, 1}, \ldots, p_{i, n}\right), i=1, \ldots, k$, correspond to the embeddings $\phi_{1}, \ldots, \phi_{k}$, i.e., for every $i$ and every $x_{j} \in X$

$$
\phi_{i}\left(x_{j}\right)=p_{i, j}\left(z_{1}, \ldots, z_{m}, y_{1}, \ldots, y_{t}, g_{1}, \ldots, g_{l}\right) .
$$

(5) Moreover, let $\Phi_{i}=\left\{\phi_{i} \sigma \eta_{i} \psi \mid \sigma \in A_{D^{*}}, \psi \in V\left(\bar{U}_{i}\right)\right\}$. Then $\Phi_{i} \subseteq p_{i}\left(V\left(U_{D, i}\right)\right)$ and

$$
V(U)=\Phi_{1} \cup \ldots \cup \Phi_{k} \cup V\left(U \wedge V_{1}\right) \cup \ldots \cup V\left(U \wedge V_{s}\right) .
$$

Proof. We have already constructed the systems

$$
U_{D, 1}=1, \ldots, U_{D, k}=1
$$

and embeddings

$$
\phi_{1}: F_{R(U)} \rightarrow F_{R\left(U_{D, 1}\right)}, \ldots, \phi_{k}: F_{R(U)} \rightarrow F_{R\left(U_{D, k}\right)} .
$$

Recall that $L_{1}, \ldots, L_{k+s}$ is a family of all standard maximal fully residually free quotients for $G$ such that for each $i=1, \ldots, k$ all the restrictions of $\pi_{i}$ onto rigid subgroups of $D$, onto edge subgroups in $D$, and onto the subgroups of the abelian vertex groups $A$ generated by the images of all the edge groups of edges adjacent to $A$, are monomorphisms. Consider now fully residually free quotients $L_{k+1}, \ldots, L_{k+s}$. For each quotient $L_{k+i}$ there exists an element $V_{i}$ from a rigid subgroup or from $A_{e}$ for some abelian vertex group $A$ such that $\pi_{i}\left(V_{i}\right)=1$. Since all automorphisms from $A_{D}$ only conjugate rigid subgroups, all solutions of $U=1$ that can be represented as a composition $\sigma \pi_{i}$, where $\sigma \in A_{D}$, satisfy the equation $V_{i}=1$.

In order to prove statements (4) and (5) we just notice that the set $\phi_{i} \sigma$, where $\sigma \in A_{D^{*}}$ is the set of canonical automorphisms from $A_{D}$ except the automorphisms corresponding to stable QH subgroups and edges between rigid subgroups for which we did not extend centralizers. Using these automorphisms one can transform an arbitrary solution into a solution in one of the maximal fully residually free quotients. 


\section{Effective free decompositions}

In this section we prove the following result, which is one of the corner stones in constructing JSJ decompositions affectively.

THEOREM 10.1. There is an algorithm which for every finitely generated fully residually free group $G$ and its finitely generated subgroup $H$ determines whether or not $G$ has a nontrivial free decomposition modulo $H$. Moreover, if $G$ does have such a decomposition, the algorithm finds one (by giving finite generating sets of the factors).

Proof. Let $G$ be a finitely generated fully residually free group, $G=\langle Z|$ $U(Z)\rangle$, where $U(Z)=1$ is an irreducible system over $F$. Let $H$ be a finitely generated subgroup of $G$ given by a finite set of generators $H=\left\langle h_{1}(Z), \ldots, h_{n}(Z)\right\rangle$, where $h_{i}(Z)$ is a word in generators $Z$. For every $i=1, \ldots, n$ add the equation $y_{i}=$ $h_{i}(X)$ to the system $U(Z)=1$. We denote the resulting system by $U_{H}(Z, Y)=1$.

Let

$$
G \simeq G_{1} * \ldots * G_{k}
$$

be a free decomposition of $G$ such that $H \leq G_{1}, G_{1}$ is freely indecomposable modulo $H$, and $G_{i}$ is a freely indecomposable non-trivial group for $i \neq 1$. We deduce from (78) that $G=F_{R(S)}$ for some irreducible system $S(X)=1$ over $F$ which splits as $S(X)=S_{1}\left(X_{1}\right) \cup \ldots \cup S_{k}\left(X_{k}\right)$; here $S_{i}\left(X_{i}\right)$ is a finite set of defining relations of $G_{i}$. Notice that the system $S_{1}\left(X_{1}\right)=1$ may contain constants from $F$, but $S_{i}\left(X_{i}\right)=1$ are coefficient free. Obviously, the system $S(X)=1$ is rationally equivalent to $U(Z)=1$ as well as to $U_{H}(Z, Y)=1$. Let $F=F(A), F_{i}$ be an isomorphic copy of $F$ and $\alpha_{i}: F \rightarrow F_{i}$ be a fixed isomorphism $(i=2, \ldots, k)$. Denote by $F^{*}$ the free product

$$
F^{*}=F_{1} * F_{2} \ldots * F_{k}
$$

in which $F_{1}=F$. For every $\mu \in \operatorname{Hom}(G, F)$ there exists $\lambda_{\mu} \in \operatorname{Hom}\left(G, F^{*}\right)$ defined for $x \in X_{i}$ as $\lambda_{\mu}(x)=\alpha_{i}\left(\left.\mu\right|_{G_{i}}(x)\right)$. Every solution of $S(X)=1$ in $F$ can be obtained by a substitution from a solution $\lambda$ such that $\lambda(x) \in F_{i}$ for $x \in X_{i}$. Since $G$ is discriminated by $F$ we can chose $\lambda$ such that $\lambda(x) \neq 1$ for any $x \in X$ (otherwise we can replace $X$ by a smaller set of generators for $G$ ).

Denote by $\phi_{\lambda}$ the solution of the system $U_{H}=1$ which is obtained from $\lambda$ by changing coordinates. Varying the solution $\lambda$ we will get a set of solutions

$$
\Phi=\left\{\phi_{\lambda} \mid \lambda\right\},
$$

which discriminates $F_{R(S)}$ into $F^{*}$. We may assume that all solutions in $\Phi$ have syllable length in $F^{*}$ not greater than a constant $K$ depending on the expressions of generators $Z$ in terms of generators $X$.

Since the set $\mathcal{G} E\left(U_{H}\right)$ of generalized equations corresponding to the system $U_{H}(Z, Y)=1$ is finite, there exists a generalized equation $\Omega_{\Phi} \in \mathcal{G} E\left(U_{H}\right)$ and the canonical embedding $\gamma: F_{R\left(U_{H}\right)} \rightarrow F_{R\left(\Omega_{\Phi}\right)}$ such that some infinite subset of $\Phi$ factors through $\gamma$ and discriminates $F_{R\left(U_{H}\right)}$. For simplicity we denote this subset again by $\Phi$ and also identify $G \simeq F_{R\left(U_{H}\right)}$ with its image $\gamma\left(F_{R\left(U_{H}\right)}\right)$ in $F_{R\left(\Omega_{\Phi}\right)}$.

We claim that given $\Omega_{\Phi}$ one can effectively construct a generalized equation $\Omega$ with the following properties:

a) there is an embedding $\phi: F_{R\left(\Omega_{\Phi}\right)} \rightarrow F_{R(\Omega)}$; 
b) the set of all closed sections of $\Omega$ is partitioned into a disjoint union of subsets $C_{1}, \ldots, C_{k}$ such that any base from $C_{i}$ has its dual base in $C_{i}$, which implies that

$$
F_{R(\Omega)} \simeq F_{R\left(\Omega_{1}\right)} * \ldots * F_{R\left(\Omega_{k}\right)}
$$

where $\Omega_{i}$ is the generalized equation situated over the section $C_{i}$;

c) $\phi\left(\gamma\left(F_{R\left(S_{i}\right)}\right)\right) \leq F_{R\left(\Omega_{i}\right)}$.

Let $\hat{T}_{\mathrm{dec}}\left(U_{H}, \Omega_{\Phi}\right)$ be the tree constructed in Subsection 9.1 Since the tree $\hat{T}_{\mathrm{dec}}\left(U_{H}, \Omega_{\Phi}\right)$ is finite there is a branch $B$ of this tree from the root $v_{0}$ to a leaf, say $w$, such that an infinite discriminating subset of homomorphisms from $\Phi$ goes along this branch. Again we denote this subset by $\Phi$. Let $e=(v, u)$ be an edge in $B, \pi_{B, u}$ the canonical homomorphism from $\Omega_{\Phi}$ into $F_{R\left(\Omega_{u}\right)}$ along the branch $B$, $\pi_{B, u}^{*}$ the restriction of $\pi_{B, u}$ onto $F_{R\left(U_{H}\right)}$, and $G(u)=\pi_{B, u}^{*}\left(F_{R\left(U_{H}\right)}\right)$. When doing the Elimination process along the branch $B$ the following cases may occur at the vertex $v$.

1) Neither a quadratic section nor a periodic structure occurs in constructing the generalized equation $\Omega_{v}$ at $v$.

2) A quadratic section or a periodic structure occurs in constructing the generalized equation $\Omega_{v}$ at $v$ and the splitting induced on $G(v)$ from $F_{R\left(\Omega_{v}\right)}$ is insufficient, i.e., the homomorphism $\pi_{B, u}^{*}: F_{R\left(U_{H}\right)} \rightarrow G(v)$ is an isomorphism.

3) A quadratic section or a periodic structure occurs in constructing the generalized equation $\Omega_{v}$ at $v$ and the splitting induced on $G(v)$ from $F_{R\left(\Omega_{v}\right)}$ is sufficient, i.e., the homomorphism $\pi_{B, u}^{*}: F_{R\left(U_{H}\right)} \rightarrow G(v)$ is not an isomorphism. Notice that this case can occur only if $u=w$.

Observe that given a branch $B$ one can effectively construct the homomorphism $\pi_{B, u}$ because the construction of the tree $\hat{T}_{\mathrm{dec}}\left(U_{H}, \Omega_{\Phi}\right)$ is effective. Also, effectiveness of the homomorphism $\gamma$ implies that one can effectively find the subgroup $F_{R\left(U_{H}\right)}$ in $\Omega_{\Phi}$ hence the homomorphism $\pi_{B, u}^{*}$. It follows that one can effectively decide which of the cases above occurs at a given vertex $v \in B$. Indeed, if the case 1 ) occurs at $v$ then it is obvious from the process. If the case 1) does not occur then to distinguish the case 2) from the case 3) one needs to check whether the homomorphism $\pi_{B, u}^{*}$ is an isomorphism or not. This can be done effectively by Theorem 3.22

It follows that one can effectively find the set $V(3)$ of all the vertices $v \in$ $\hat{T}_{\mathrm{dec}}\left(U_{H}, \Omega_{\Phi}\right)$ for which the third case holds. There are two cases here. Considering these cases we identify $G$ with its image $G(v)$.

Case 1. Suppose there is no vertex from $V(3)$ in the branch $B$. In this event either the vertex $v=w$ is a leaf and it has type 2 (in the tree $T\left(\Omega_{\Phi}\right)$ ) or $\Omega_{w}$ is obtained from $\Omega_{v}$ by a second minimal relacement and $w$ has type 2 . Therefore, in the equation $\Omega_{w}$ all the bases are located in the non-active part. Observe that by the construction of the tree $\hat{T}_{\mathrm{dec}}\left(U_{H}, \Omega_{\Phi}\right)$ the non-active part of $\Omega_{w}$ does not have periodic structures. Hence it coincides with the parametric part. There are several cases to consider. Considering these cases we identify $G$ with its image $G(w)$.

Case 1.1. All the pieces of the bases corresponding to the generators $Z$ of $G$ in $\Omega_{\Phi}$ are eventually transferred to the parametric part of $\Omega_{w}$. In this case the group $G$ is freely indecomposable modulo $H$. Indeed, observe first that each homomorphism $\phi$ from $\Phi$ maps all the items from the parametric part of $\Omega_{w}$ to the subgroup $F_{1}$ 
of $F^{*}$. This follows from the fact that $\phi(H) \leq F_{1}$ and the property that solutions of generalized equations are reduced as written, so the image under $\phi$ of a piece of a base corresponding to a generator, say $h$ of $H$ occurs in $\phi(h)$ as a subword, so it again belongs to $F_{1}$. This implies that $\phi(G) \leq F_{1}$ for every $\phi \in \Phi$ - hence $G=G_{1}$, thus $G$ is freely indecomposable.

Case 1.2. Not all pieces of the bases corresponding to the generators $Z$ of $G$ in $\Omega_{\Phi}$ are eventually transferred to the parametric part of $\Omega_{w}$ (some of them were deleted as matched bases). In this case $F_{R\left(\Omega_{w}\right)}$ is a non-trivial free product of a free group $F_{w}$ generated by free variables of $\Omega_{w}$ and the subgroup $H_{w}$ generated by the items in the parametric part of $\Omega_{w}$.

Case 1.2a. If $G$ is not elliptic in the splitting $F_{R\left(\Omega_{w}\right)}=F_{w} * H_{w}$ then it has a non-trivial induced decomposition and one can find it effectively by Theorem 3.17 This finishes the proof of the theorem in this case.

Case 1.2 b. If $G$ is conjugated into $F_{w}$ then by Theorem 3.14 one can effectively verify this and find a conjugator. In this event one has an effective embedding of $G$ into a free group $F_{w}$, so $G$ is free and using the standard techniques from free groups it possible to find a basis of $G$, thus proving the theorem.

Case 1.2c. If $G$ is conjugated into $H_{w}$ (in this case $G \leq H_{w}$ ) then the coordinate group of the generalized equation $\Omega_{w, H}$ which is located on the parametric part of $\Omega_{w}$ is a proper quotient of the group $F_{R\left(\Omega_{w}\right)}$. In this case we have an effective embedding of $G$ into $F_{R\left(\Omega_{w, H}\right)}$ and induction finishes the proof.

Case 2. Suppose now that there is a vertex $v$ as in the case 3) in the branch $B$. The group $F_{R\left(\Omega_{v}\right)}$ admits a sufficient splitting $D$. Recall that the set of the canonical automorphisms $A_{D}$ of the splitting $D$ consists of canonical Dehn twists related to some elementary $\mathbb{Z}$-splittings or abelian splittings from $D$. Considering this case we again identify $G$ with its image $G(v)$. Let $\sigma$ be a quadratic section or a section corresponding to a periodic structure which occurs in $\Omega_{v}$. If $\sigma$ is quadratic, we add a non-active "duplicate" $\sigma^{*}$ of $\sigma$ to the generalized equation $\Omega_{v}$. Namely, we add a copy $\sigma^{*}$ of the section $\sigma$ to the end of the equation $\Omega_{v}$, make $\sigma^{*}$ non-active, and for each non-variable base $b$ in $\sigma$ identify the duplicate $b^{*}$ of $b$ in $\sigma^{*}$ with $b$ by remembering a graphical equality $b=b^{*}$. If $\sigma$ corresponds to a periodic structure, we take as $\sigma^{*}$ a section defining the corresponding extension of a centralizer, $\sigma^{*}$ in this case is an abelian section. Denote this equation $\Omega_{v, \sigma}$. For a minimal solution with respect to $A_{D}$ it will be transformed into some $\Omega_{w, \sigma}$, and the equality $b=b^{*}$ will be transformed into some expression without cancellation of $b^{*}$ as a product of bases of $\Omega_{w}$. Construct a new "bookkeeping" tree $T_{b o o k}\left(U_{H}, \Omega_{\Phi}\right)$ the following way. First we construct an auxiliary tree $T_{0, \text { book }}\left(U_{H}, \Omega_{\Phi}\right)$. The initial vertex of this tree is the same as the initial vertex of $\hat{T}_{\mathrm{dec}}\left(U_{H}, \Omega_{\Phi}\right)$. All the other vertices will be terminal vertices. Let $w$ be a terminal vertex of $T_{\mathrm{dec}}\left(U_{H}, \Omega_{\Phi}\right)$. Let $v$ be a vertex that precedes $w$. To each such vertex $v$ we assign some quadratic section $\sigma$ for which a minimal solution with respect to canonical automorphisms corresponding to this section satisfies a proper equation or a section corresponding to a periodic structure in $\Omega_{v}$. If $\sigma$ is a quadratic section, let $\Omega_{v}^{\prime}$ be a generalized equation obtained from $\Omega_{v}$ by deleting $\sigma$ from $\Omega_{v}$. Notice that the complexity of $\Omega_{v}^{\prime}$ is strictly less than the complexity of $\Omega_{v}$. Let $\Omega_{v, \sigma}^{\prime}$ be obtained from $\Omega_{v, \sigma}$ by deleting the quadratic section $\sigma$. If $\sigma$ is a section corresponding to a periodic structure, then we assign to the terminal vertex of $T_{0, \text { book }}\left(U_{H}, \Omega_{\Phi}\right)$ the generalized equation $\Omega_{w, \sigma}$. If $\sigma$ is quadratic, then we assign to the terminal vertex of $T_{0, \text { book }}\left(U_{H}, \Omega_{\Phi}\right)$ the generalized equation 
$\Omega_{v, \sigma}^{\prime}$. To obtain $T_{\text {book }}\left(U_{H}, \Omega_{\Phi}\right)$ we first construct $T_{0, \text { book }}\left(U_{H}, \Omega_{\Phi}\right)$. If for a terminal vertex of $T_{0, \text { book }}\left(U_{H}, \Omega_{\Phi}\right) \sigma$ corresponds to a periodic structure, then we paste to this vertex $T_{0, \text { book }}\left(U_{H}, \Omega_{w, \sigma}\right)$. If a terminal vertex of $T_{0 \text {,book }}\left(U_{H}, \Omega_{\Phi}\right)$ has quadratic section $\sigma$, then we paste to this vertex $T_{0 \text {,book }}\left(U_{H}, \Omega_{v, \sigma}^{\prime}\right)$. We iterate this process. The tree $T_{b o o k}\left(U_{H}, \Omega_{\Phi}\right)$ is finite because on each step of the construction we either decrease the complexity of the generalized equation from the previous step or we do not increase the complexity and the group generated by all the variables except the ones on the sections $\sigma^{*}$ is a proper quotient of such group on the previous step.

If $\Phi$ is constructed using infinitely many Dehn twists related to an edge group $\langle u\rangle \leq F_{R\left(\Omega_{v}\right)}$, then $u \in G_{v}$. Moreover, because of our choice of the set of homomorphisms $\Phi$, the element $u$ has to belong to one of the free factors $\pi_{B}\left(G_{i}\right)$ of $G_{v}$, which are homomorphic images of the initial free factors of $G$. Indeed, suppose that the cyclic syllable length of $u$ in $G_{v}$ is more than 1, then, applying sufficiently many Dehn twists related to $u$, one can obtain an element of arbitrary large syllable length. In this event, applying automorphisms from $A_{D}$, we can obtain a set of solutions with arbitrary syllable length in $F$, which contradicts to the choice of $\Phi$.

Let $\sigma$ be a quadratic section for $F_{R\left(\Omega_{v}\right)}$ and let $Q$ be the corresponding $\mathrm{QH}$ subgroup of $F_{R\left(\Omega_{v}\right)}$. Denote by $\Omega_{v}^{\prime}$ the generalized equation obtained from $\Omega_{v}$ by removing $\sigma$. Then the complexity of $\Omega_{v}^{\prime}$ is strictly less than the complexity of $\Omega_{v}$. By induction we can decide if $F_{R\left(\Omega_{v}^{\prime}\right)}$ can be represented as in b). Represent $F_{R\left(\Omega_{v}\right)}$ as a fundamental group of a graph of groups with one vertex group $Q$ and the others subgroups of $F_{R\left(\Omega_{v}^{\prime}\right)}$. Denote this decomposition by $D_{1}$. By Theorem 3.17 we can find effectively the induced decomposition $D_{1}(G)$ of $G$.

The following cases occur.

Case 2.1. $G$ is conjugated into $F_{R\left(\Omega_{v}^{\prime}\right)}$. In this case we apply induction.

Case 2.2. $G$ is conjugated into $Q$, then $G$ is free.

Case 2.3. $G$ is not conjugated into $F_{R\left(\Omega_{v}^{\prime}\right)}$ and all the conjugates of $Q^{q}$ have the intersection $Q^{q} \cap G$ of infinite index in $Q^{q}$. Then by the corollary from Lemma 3.14 $G$ is freely decomposable modulo $H$, and the decomposition $D_{1}(G)$ which can be found effectively by Theorem 3.17 is a free decomposition.

Case 2.4. Some conjugate of $Q^{q}$ has the intersection $Q^{q} \cap G$ of finite index in $Q^{q}$. Without loss of generality we can suppose $Q_{G}=Q \cap G$ is of finite index in $Q$. As a group of a surface with boundaries, $Q_{G}$ is freely indecomposable modulo the edge groups. Let $D_{2}(G)$ be a free decomposition of $G$ modulo $H$. Either $Q_{G}$ is a $\mathrm{QH}$ subgroup of one of the factors in the decomposition $D_{2}(G)$, or one of the edge groups in $Q_{G}$ is hyperbolic in $D_{2}(G)$. In the second case all other edge groups can be all together included into a basis of the free group $Q_{G}$, they generate a free factor in the decomposition of $Q_{G}$. The other factor in the free decomposition of $Q_{G}$ is a free factor in a free decomposition of $G$. In this case a minimal solution of $U_{H}=1$ with respect to canonical automorphisms of $Q_{G}$ does not satisfy a proper equation and we can pass to this minimal solution. G.

Obviously, all abelian subgroups of $G$ are elliptic in any free decomposition of

To finish case 3) apply induction to the generalized equation in the terminal vertex of $T_{0 \text {,book }}\left(U_{H}, \Omega_{\Phi}\right)$. Going along some branch of the tree $T_{\text {book }}\left(U_{H}, \Omega_{\Phi}\right)$ we either obtain a free decomposition of $G$ as one of the induced decompositions considered in the process or arrive to a generalized equation, in which the set of 
closed active and parametric sections is partitioned into disjoint subsets of independent sections described above. Adjoining the sections from $\sigma^{*}$, we suppose that they all have syllable length one, so if the expressions for constant bases from $\sigma^{*}$ include variables from several independent close sections on the next level of the tree $T_{\text {book }}\left(U_{H}, \Omega_{\Phi}\right)$, we join these sections into the same component. Going from the leaf vertices of this tree to the initial vertex, we obtain the generalized equation $\Omega$ with the properties a)-c). If in a)-c) we have $k=1$ for all the branches of $T_{\text {book }}\left(U_{H}, \Omega_{\Phi}\right)$, then $G$ is freely indecomposable modulo $H$.

COROLLARY 10.2. There is an algorithm which for every finitely generated fully residually free group $G$ finds its Grushko decomposition.

COROLLARY 10.3. There is an algorithm which for every finitely generated fully residually free group $G$ and its finitely generated subgroup $H$ finds a free decomposition of $G$ modulo $H$

$$
G \simeq G_{1} * \cdots * G_{n}
$$

such that $H \leq G_{1}$ and $G_{1}$ is freely indecomposable modulo $H, G_{i}$ is freely indecomposable non-trivial group for every $i=2, \ldots, n$.

COROLlary 10.4. There is an algorithm which for every finitely generated fully residually free group $G$ and a family of finitely generated subgroups $H_{1}, \ldots, H_{m}$ finds a reduced compatible free decomposition of $G$ modulo $\left\{H_{1}, \cdots, H_{m}\right\}$

$$
G \simeq G_{1} * \cdots * G_{n}
$$

such that $H_{1} \leq G_{1}$. This decomposition of $G$ may be trivial.

Proof. Notice that since conjugates of different factors in a free decomposition intersect trivially, a subgroup can be conjugated only into one factor in a given decomposition. We try all possible combinations for subgroups that can be conjugated into the same factor as $H_{1}$. Suppose they are $H_{1}, \ldots, H_{s}$. Let $H_{i}$ be generated by $h_{i 1}, \ldots, h_{i j_{i}}$. We represent $h_{i k}=g_{i}^{-1} \bar{h}_{i k} g_{i}$ for $k=2, \ldots, j_{i}$ and put all the elements $h_{1 k}, k=1, \ldots, i_{1}^{\prime}, \bar{h}_{i k}, i=1, \ldots s, k=1, \ldots, j_{i}$ into the parametric part of the initial generalized equations. We include the case when some of $g_{i}$ 's are trivial. As in the proof of the theorem, we can find a free decomposition of $G$ such that all $H_{1}, \ldots, H_{s}$ are conjugated into the same factor, and $H_{1}$ belongs to this factor. If the obtained decomposition is non-trivial, apply induction.

THEOREM 10.5. There is an algorithm which for every finitely generated fully residually free group $G$ determines whether $G$ is free or not, and if it is the algorithm finds a free basis of $G$.

PROOF. Let $G$ be a finitely generating fully residually free group. By Corollary 10.2 one can effectively find a Grushko decomposition of $G$ :

$$
G=G_{1} * \ldots * G_{m}
$$

so the factors $G_{i}$, which are given by finite sets of generators $G_{i}=\left\langle Y_{i}\right\rangle$, are freely indecomposable. By Theorem 3.21 one can find finite presentations of the groups $G_{i}$. Since the word problem is decidable in every group $G_{i}$ one can effectively verify whether each one of the groups $G_{i}$ is abelian or not. If one of the groups $G_{i}$ is nonabelian then $G$ is not free. Otherwise, one has to check if all the abelian groups $G_{i}$ are cyclic or not. This can be done algorithmically since the finitely generated abelian groups $G_{i}$ are given by their finite presentations. If one of them is not cyclic 
then $G$ is not free, and if all of them are cyclic then $G$ is a free product of infinite cyclic groups, hence free. This proves the theorem.

\section{Homomorphisms of finitely generated groups into fully residually free groups}

11.1. Hom-diagrams. Let $G$ and $H$ be finitely generated groups. Recall that by $\operatorname{Hom}(G, H)$ we denote the set of all homomorphisms from $G$ into $H$. If $G$ contains $H$ as a fixed distinguished subgroup then by $\operatorname{Hom}_{H}(G, H)$ we denote the set of $H$-homomorphisms from $G$ onto $H$ (the set of retracts from $G$ into $H$ ). In this section for a free non-abelian group $F$ we describe the set $\operatorname{Hom}_{F}(G, F)$ as well as $\operatorname{Hom}(G, F)$ in terms of hom-diagrams.

Let $T$ be a rooted directed tree with the set of vertices $V=V(T)$ and the set of edges $E=E(T)$. Suppose that for every vertex $v \in V$ there is a group $G_{v}$ and a group of automorphisms $A_{v} \leq \operatorname{Aut}\left(G_{v}\right)$ associated with $v$, and with every directed edge $e=(u, v) \in E$ there is a homomorphism $\pi_{u, v}: G_{u} \rightarrow G_{v}$ associated with $e$. We call this structure a hom-diagram. Suppose a group $G=G_{v_{0}}$ is assigned to the root vertex $v_{0}$ in $T$. Then every branch (directed path) $B$ in $T$ from $v_{0}$ to a leaf vertex $v_{n}$

$$
B: v_{0} \rightarrow v_{1} \rightarrow \ldots \rightarrow v_{n}
$$

determines a set of homomorphisms $\Phi_{B} \subseteq \operatorname{Hom}\left(G, G_{v}\right)$ as follows. Put

$$
A_{B}=A_{v_{0}} \times \ldots \times A_{v_{n}} .
$$

If $\sigma=\left(\sigma_{0}, \ldots, \sigma_{n}\right) \in A_{B}$ then

$$
\phi_{B, \sigma}=\sigma_{0} \pi_{v_{0}, v_{1}} \sigma_{2} \pi_{v_{1}, v_{2}} \ldots \pi_{v_{n-1}, v_{n}} \sigma_{n}
$$

is a homomorphism from $\operatorname{Hom}\left(G, G_{v_{n}}\right)$. Set

$$
\Phi_{B}=\left\{\phi_{B, \sigma} \mid \sigma \in A_{B}\right\} .
$$

Now assume that a group $H$ is given and for every leaf vertex $v$ in $T$ the group $G_{v}$ associated with $v$ is a free product $G_{v}=H_{v} * F_{v}$ of a subgroup $H_{v}$ of $H$ and a free group $F_{v}=F_{v}\left(X_{v}\right)$ with basis $X_{v}$. We denote such diagrams by $\mathcal{C}=\mathcal{C}(T, G, H)$ and refer to them as to $\operatorname{Hom}(G, H)$-diagrams. If $v$ is a leaf vertex of $T$ then an embedding $\lambda: H_{v} \rightarrow H$ together with a map $\xi: X_{v} \rightarrow H$ give rise to the canonical homomorphism

$$
\psi_{\lambda, \xi}: G_{v} \rightarrow H
$$

It follows that the branch $B$, and a triple $\sigma, \lambda, \xi$ determine a homomorphism $\phi_{B, \sigma, \lambda, \xi}=\phi_{B, \sigma} \psi_{\lambda, \xi}$ which is composition of $\phi_{B, \sigma}$ and $\psi_{\lambda, \xi}$ :

$$
\phi_{B, \sigma, \lambda, \xi}: G \rightarrow H \text {. }
$$

The set $\Phi_{\mathcal{C}, B}$ of all such homomorphisms is called the fundamental sequence of the hom-diagram $\mathcal{C}$ determined by the branch $B$. Union of all fundamental sequences of the hom-diagram $\mathcal{C}$ is denoted by

$$
\mathcal{C} H \operatorname{Hom}(G, H)=\cup\left\{\Phi_{\mathcal{C}, B} \mid B \text { is a branch of } T\right\} \text {, }
$$

we refer to it as to the set of all homomorphisms from $\operatorname{Hom}(G, H)$ determined by $\mathcal{C}$. If $\mathcal{C H o m}(G, H)=\operatorname{Hom}(G, H)$ then we say that the hom-diagram $\mathcal{C}(T, G, H)$ is complete.

We say that a hom-diagram $\mathcal{C}$ is canonical if the following conditions are satisfied: 
1) all groups $G_{v}$ associated with vertices in $T$ are fully residually $H$, except, perhaps, for the group $G$ associated with the root vertex $v_{0}$;

2) all homomorphisms $\pi_{e}$ associated with edges of $T$ are surjective and proper (i.e., with a non-trivial kernel);

3 ) the groups of automorphisms $A_{v}$ are canonical with respect to some JSJ decomposition of free quotients in a Grushko decomposition of $G_{v}$ (see Section 2).

In the case where $H$ is a fixed subgroup of $G$ one can consider only the set of retracts $\operatorname{Hom}_{H}(G, H)$. In this event we assume that in the corresponding homdiagram $\mathcal{C}$ all homomorphisms assigned to edges in $T$ are $H$-homomorphisms, the vertex groups $G_{v}$ associated with leaves $v$ in $T$ are isomorphic to $H * H_{v} * F_{v}$, where $H_{v}$ is a subgroup of $H$, and the mappings $\lambda: H * H_{v} \rightarrow H$ are identical on $H$ and embeddings on $H_{v}$. In this case we say that $\mathcal{C}$ is $H$-complete or $\operatorname{Hom}_{H}(G, H)$ complete if $\mathrm{Hom}_{H}(G, H)=\mathrm{CHom}_{H}(G, H)$.

We say that a hom-diagram can be found effectively if all groups $G_{v}$ are given by some finite presentations, for all groups $A_{v}$ particular finite generated sets are given, all homomorphisms $\pi_{e}$ and the automorphisms generating $A_{v}$ are given by their images on the corresponding finite generating sets.

11.2. Description of homomorphisms from a finitely generated fully residually free groups into a free non-abelian group. Let $G$ be a finitely generated fully residually free group. In this section we describe effectively the sets $\operatorname{Hom}(G, F)$ and $\operatorname{Hom}_{F}(G, F)$ in terms of hom-diagrams.

TheOREm 11.1. Let $G$ be a group from $\mathcal{F}$ and $F$ a free group. Then:

(1) there exists a complete canonical $\operatorname{Hom}(G, F)$-diagram $\mathcal{C}$.

(2) if $F$ is a fixed subgroup of $G$ then there exists an $F$-complete canonical $\operatorname{Hom}_{F}(G, F)$-diagram $\mathcal{C}$.

Moreover, the Hom-diagrams from 1) and 2) can be found effectively.

Proof. Since $G$ is fully residually free, it can we represented as $G=F_{R(U)}$ for the irreducible system $U=1$ over $F$. We construct an embedding tree $T_{\text {emb }}(G)$ by induction. Observe from the beginning that $T_{e m b}(G)$ is a rooted tree oriented from the root $v_{0}$. We associate the group $G$ with $v_{0}$. Let $\bar{U}_{i}, i=1, \ldots, t$ be the systems corresponding to $U=1$ from Theorem 9.3. We add new vertices $v_{11}, \ldots, v_{1 t}$ and connect $v_{0}$ with each $v_{1 i}$ by an edge $e_{1 i}$. We associate the group $F_{R\left(\bar{U}_{i}\right)} *$ $F\left(t_{1}, \ldots, t_{k_{i}}\right)$ with the vertex $v_{1 i}$, and the canonical epimorphism $\pi_{1 i}: F_{R(U)} \rightarrow$ $F_{R\left(\bar{U}_{i}\right)} * F\left(t_{1}, \ldots, t_{k_{i}}\right)$ with the edge $e_{1 i}, i=1, \ldots, t$. By Corollary 10.2 one can find a Grushko's decomposition of $F_{R\left(\bar{U}_{i}\right)}$ effectively. Notice, that freely indecomposable non-abelian factors in the Grushko's decomposition of $F_{R\left(\bar{U}_{i}\right)}$ again satisfy Theorem 9.3 This allows one to continue the construction of a tree by induction. The resulting rooted tree is denoted by $T_{e m b}(G)$, we refer to it as to the embedding tree. By construction, every vertex in $T_{e m b}(G)$ has only finitely many outgoing edges. We claim that this tree is finite. Indeed, since the group $F$ is equationally Noetherian it follows that there are no infinite sequences of proper surjective homomorphisms of irreducible coordinate groups. Now the claim follows from Koenig's lemma.

By construction $G$ is embedded into the NTQ group corresponding to each branch of $T_{e m b}(G)$. The solution tree $T=T_{\text {sol }}(U)$ which in an underlying tree of the Hom-diagram for $\operatorname{Hom}(G, F)$ or $\operatorname{Hom}_{F}(G, F)$ can be obtained from $T_{\text {emb }}(G)$ 
by adding edges connecting $v_{0}$ to the initial vertices of $T_{e m b}\left(F_{R\left(U \wedge V_{i}\right)}\right)$ for each equation $V_{i}=1$ from Theorem 9.3 and adding similar edges on each level of the construction. The groups of automorphisms $A_{v}$ assigned to vertices of $T_{\text {sol }}(U)$ are canonical groups of automorphisms corresponding to JSJ decompositions of these groups. By Theorem 9.3 the Hom- diagram corresponding to $T_{\text {sol }}(U)$ is complete and canonical.

11.3. Hom-diagrams of finitely generated groups. In 1987 Razborov proved the following remarkable result 62: for every finitely generated group $G$ and a free non-abelian group $F$ one can effectively find a complete constructive hom-diagram $\mathcal{C}=\mathcal{C}(T, G, F)$. In his diagram the associated groups were not, in general, fully residually free (only residually free), the homomorphisms were not necessary onto, and the groups of automorphisms were not canonical. In the paper 36 the authors rewrote the Razborov's elimination process which allowed them to show that all the associated groups $G_{v}$ are subgroups of the coordinate groups of NTQ-systems, hence fully residually free. Still the diagram was far from being canonical.

The following is a rephrasing of Theorem 5 from the preprint 39 .

TheOREm 11.2. Let $G$ be a finitely generated group $G$ and $F$ a free group. Then:

(1) there exists a complete canonical $\operatorname{Hom}(G, F)$-diagram $\mathcal{C}$.

(2) if $F$ is a fixed subgroup of $G$ then there exists an $F$-complete canonical $\operatorname{Hom}_{F}(G, F)$-diagram $\mathcal{C}$.

Moreover, if the group $G$ is finitely presented then the Hom-diagrams from 1) and 2) can be found effectively.

PRoOF. It follows directly from Theorem 9.3 and the construction of $T_{\text {sol }}$ in Subsection 11.2

11.4. Solutions of systems of equations in free groups and homdiagrams. In this section we describe effectively all solutions of a finite system of equations in a free non-abelian group $F$.

THEOREM 11.3. Let $S(X)=1$ be a finite system of equations and $G=(F * F[X]) / n c l(S)$. Then one can effectively construct an $F$-complete canonical $\operatorname{Hom}(G, F)$-diagram $\mathcal{C}$ such that all solutions of $S(X)=1$ in $F$ are exactly all $F$ homomorphisms in $\mathcal{C}$.

\section{Free Lyndon length functions on NTQ groups.}

12.1. Lyndon length functions. In paper [48 Lyndon introduced an abstract notion of a length function on a group, which gives an axiomatic approach to Nielsen cancellation argument. In this section we briefly discuss length functions on groups.

Let $G$ be a group and $A$ an ordered abelian group. Then a function $l: G \rightarrow A$ is called a (Lyndon) length function on $G$ if the following conditions hold:

(L1) $\forall x \in G: l(x) \geq 0$ and $l(1)=0$;

(L2) $\forall x \in G: l(x)=l\left(x^{-1}\right)$;

(L3) $\forall x, y, z \in G: c(x, y)>c(x, z) \rightarrow c(x, z)=c(y, z)$, where $c(x, y)=\frac{1}{2}\left(l(x)+l(y)-l\left(x^{-1} y\right)\right)$. 
In general $c(x, y) \notin A$, but $c(x, y) \in A_{\mathbb{Q}}$, where $A_{\mathbb{Q}}$ is a $\mathbb{Q}$-completion of $A$, so in the axiom (L3) we view $A$ as a subgroup of $A_{\mathbb{Q}}$.

Below we list several extra axioms which describe some special classes of Lyndon length functions. Sometimes we write $x_{1} \circ \ldots \circ x_{n}$ instead of $x_{1} \ldots x_{n}$ if $l\left(x_{1} \ldots x_{n}\right)=$ $l\left(x_{1}\right) \circ \ldots \circ l\left(x_{n}\right)$.

(L4) $\forall x \in G: c(x, y) \in A$.

A length function $l: G \rightarrow A$ is called free, if it satisfies

(L5) $\forall x \in G: x \neq 1 \rightarrow l\left(x^{2}\right)>l(x)$.

A group $G$ has a Lyndon length function $l: G \rightarrow A$, which satisfies axioms (L4)(L5) if and only if $G$ acts freely on some $A$-tree. This is a remarkable result due to Chiswell (see [15]).

A length function $l: G \rightarrow A$ is called regular if it satisfies the following regularity axiom:

(L6) $\forall x, y \in G, \exists u, x_{1}, y_{1} \in G$ :

$$
x=u \circ x_{1} \& y=u \circ y_{1} \& l(u)=c(x, y) .
$$

The regularity condition is crucial for Nielsen's cancellation method, which is the base for generalized equations and elimination process from Sections 4 and 5 for solving equations over $F$.

12.2. Free regular Lyndon length functions on HNN-extensions. In this section we will show how to extend a free regular length function given on a group $H \in \mathcal{F}$ onto an HNN extension of $H$ of a particular type in such a way that the extended length function is again free and regular.

Let $H$ be a group from $\mathcal{F}, \Lambda$ an ordered abelian group, and $\ell_{H}: H \rightarrow \Lambda$ a free regular length function on $H$.

Suppose that

$$
G=\left\langle H, x \mid x^{-1} v x=u\right\rangle,
$$

where elements $u, v \in H$ satisfy the following conditions:

1) $\ell_{H}(u)=\ell_{H}(v)$;

2) $u, v$ are cyclically reduced, i.e., $u^{2}=u \circ u, v^{2}=v \circ v$, and not proper powers;

3) the subgroups $\langle u\rangle$ and $\langle v\rangle$ are conjugately separated in $H$, i.e., $\langle u\rangle^{h} \cap\langle v\rangle=$ 1 for every $h \in H$.

Define a function

$$
\ell_{G}: G \rightarrow \Lambda_{0}=\Lambda \oplus \mathbb{Z}
$$

as follows. Suppose $g \in G$ is given in its reduced form (as an element of the HNN extension $G$ )

$$
g=g_{1} x^{\alpha_{1}} g_{2} \ldots g_{m} x^{\alpha_{m}} g_{m+1} .
$$

For $n \in \mathbb{N}$ define

$$
\ell_{1}(g, n)=\ell_{H}\left(g_{1}\left(v^{n} u^{n}\right)^{\varepsilon\left(\alpha_{1}\right)} g_{2} \ldots g_{m}\left(v^{n} u^{n}\right)^{\varepsilon\left(\alpha_{m}\right)} g_{m+1}\right)-m \ell_{H}\left(v^{n} u^{n}\right),
$$

where $\varepsilon\left(\alpha_{k}\right)=\left\{\begin{array}{r}1, \quad \text { if } \alpha_{k}>0, \\ -1, \quad \text { if } \alpha_{k}<0\end{array}\right.$ 
It has been shown in [56, 53 that for every $g \in G$ there exists a positive integer $n_{0}=n_{0}(g)$ such that $\forall n \geq n_{0} \ell_{1}(g, n)=\ell_{1}\left(g, n_{0}\right)$. Put $\ell_{1}(g)=\ell_{1}\left(g, n_{0}(g)\right)$ and define

$$
\ell_{G}(g)=\left(\ell_{1}(g), \sum_{i=1}^{m}\left|\alpha_{i}\right|\right) .
$$

Then the function $\ell_{G}: G \rightarrow \Lambda \oplus \mathbb{Z}$ is well-defined.

The following result follows from [56 and [53, a more detailed version is contained in 43 .

THEOREM 12.1. Let $H$ be a group from $\mathcal{F}$ with a free regular length function $\ell_{H}: H \rightarrow \Lambda$ and

$$
G=\left\langle H, x \mid x^{-1} v x=u\right\rangle
$$

an $H N N$ extension of $H$ of the type (79). Then the function

$$
\ell_{G}: G \rightarrow \Lambda \oplus \mathbb{Z}
$$

constructed above, is a free regular length function on $G$.

12.3. Coordinate groups of regular quadratic equations. Let $G$ be a finitely generated freely indecomposable fully residually free group. Let $U=1$ be an irreducible system over $F$, such that $G=F_{R(U)}$. Let $Q$ be a MQH subgroup of $G$. Consider a relation

$$
\prod_{i=1}^{n}\left[x_{i}, y_{i}\right] \prod_{j=1}^{k} c_{j}^{z_{j}} d^{-1}=1
$$

or

$$
\prod_{r=1}^{n} x_{r}^{2} \prod_{l=1}^{k} c_{l}^{z_{l}} d^{-1}=1
$$

corresponding to $Q$. We define the basic sequence

$$
\Gamma=\left(\gamma_{1}, \gamma_{2}, \ldots, \gamma_{K(m, n)}\right)
$$

of canonical automorphisms of the group $G$ corresponding to the vertex group $Q$ the same way as we did for the corresponding quadratic equation. A family of solutions $\Psi=\left\{\phi \beta \mid \phi \in \Gamma_{P}\right\}$ for $U=1$, where $\beta$ is an arbitrary solution of $U=1$ in general position for $Q$ and $\Gamma_{P}$ is a positive unbounded family of canonical automorphisms of $H$ is called positive unbounded with respect to $Q$.

Take a solution $\beta: G \rightarrow F$ in general position with respect to $Q$. Denote by $S_{\beta}=1$ an equation

$$
\prod_{i=1}^{n}\left[x_{i}, y_{i}\right] \prod_{j=1}^{k} c_{j}^{\beta z_{j}} d^{-\beta}=1
$$

corresponding to the subgroup $Q$. Take a positive unbounded family of solutions for the equation $S_{\beta}=1$ and consider a generalized equation $\Omega=\Omega_{S_{\beta}}$ for this family of solutions.

LEMMA 12.2. The group $F_{R(\Omega)}$ is a free product of a group isomorphic to $F_{R\left(S_{\beta}\right)}$ and a free group. 
Proof. By the implicit function theorem (45], Theorem A) there is a surjective $F_{R\left(S_{\beta}\right)}$-homomorphism $\theta: F_{R(\Omega)} \rightarrow F_{R\left(S_{\beta}\right)}$. We can represent $F_{R(\Omega)}=$ $F_{R(S)} * F_{1}$ for some quadratic equation $S=1$ and free group $F_{1}$, therefore $F_{R\left(S_{\beta}\right)}$ is isomorphic to a subgroup of finite index of $F_{R(S)}$, and the retract is an isomorphism between $F_{R(S)}$ and $F_{R\left(S_{\beta}\right)}$. Hence $F_{R(\Omega)}$ in the variables of $\Omega$ is a free product of a group isomorphic $F_{R\left(S_{\beta}\right)}$ and a free group with some generators $h_{i_{1}}, \ldots, h_{i_{p}}$.

Assign to the free generators values corresponding to a minimal solution of $\Omega$. Denote by $\Omega^{\prime}$ the new generalized equation. Then the coordinate group of $F_{R\left(\Omega^{\prime}\right)}$ is isomorphic to $F_{R\left(S_{\beta}\right)}$.

LEMMA 12.3. For any positive number $N$ there is a solution of $\Omega^{\prime}$ such that one pair of variable bases in longer than $N$ times the maximal length of other variable bases.

Proof. Fix some solution of $\Omega^{\prime}$. We can make one of the pairs of variable bases of $\Omega^{\prime}$ much longer than all the others by pre-composing this solution with a power of a suitable automorphism of $F_{R\left(\Omega^{\prime}\right)}$.

Now we apply the entire transformation and bring $\Omega^{\prime}$ into the overlapping form $\Omega^{\prime \prime}$ (see Definition 6.16).

LEMMA 12.4. Generalized equation $\Omega^{\prime \prime}$ does not have open boundaries.

PRoOF. If $\Omega^{\prime \prime}$ had an open boundary, the coordinate group would split as a free product modulo $F$. This is not the case.

Equation $\Omega^{\prime \prime}$ consists of one closed section and this section is partitioned as $\mu \lambda_{1} \ldots \lambda_{n}=\Delta\left(\lambda_{i_{1}}\right) \ldots \Delta\left(\lambda_{i_{s}}\right) \Delta(\mu)$ where some of the bases are quadraticcoefficient bases. Since $\Omega^{\prime \prime}$ does not have open boundaries, the group $F_{R\left(\Omega^{\prime \prime}\right)} \approx$ $F_{R\left(S_{\beta}\right)}$ can be generated by $F$ and the bases of $\Omega$ and has relations:

$$
\mu \lambda_{1} \ldots \lambda_{n}=\Delta\left(\lambda_{i_{1}}\right) \ldots \Delta\left(\lambda_{i_{s}}\right) \Delta(\mu), \mu=\Delta(\mu), \lambda_{i}=\Delta\left(\lambda_{i}\right)
$$

for each $\lambda_{i}$. It is, therefore, generated by $F$ and the set of representatives of dual bases and has in these generators just one defining relation

$$
\mu \lambda_{1} \ldots \lambda_{n}=\lambda_{i_{1}} \ldots \lambda_{i_{s}} \mu .
$$

Denote by $F[\lambda]$ a free group generated by $F$ and all the bases $\lambda_{i}$. We proved the following

LEMMA 12.5. The group $F_{R\left(S_{\beta}\right)}$ is an $H N N$ extension of $F[\lambda]$ with stable letter $\mu$ and relation

$$
\mu \lambda_{1} \ldots \lambda_{n} \mu^{-1}=\lambda_{i_{1}} \ldots \lambda_{i_{s}} .
$$

12.4. Free regular Lyndon length functions on coordinate groups of regular quadratic equations. In this section we will construct a free regular Lyndon length function $\ell_{\beta}$ from $F_{R\left(S_{\beta}\right)}$ into $Z^{2}$ corresponding to $\Omega^{\prime \prime}$. Here $Z^{2}$ is ordered lexicographically from the right.

In the case of the group $F_{R\left(S_{\beta}\right)}$ the function $\ell_{1}$ from Section 12.2 is just the ordinary length of a reduced word in the free group $F[\lambda]$. In particular for each variable base $\lambda_{i}$ we have $\ell_{\beta}\left(\lambda_{i}\right)=\ell_{1}\left(\lambda_{i}\right)=(1,0)$. The base $\mu$ is infinitely longer than $\lambda_{i}$, namely $\ell_{\beta}(\mu)=(0,1)$. Futhermore, $\ell_{\beta}\left(\lambda_{i_{1}} \ldots \lambda_{i_{s}}\right)=\ell_{\beta}\left(\lambda_{1} \ldots \lambda_{n}\right)$ and $c\left(\mu,\left(\lambda_{i_{1}} \ldots \lambda_{i_{s}}\right)^{k}\right)=k \ell_{\beta}\left(\lambda_{i_{1}} \ldots \lambda_{i_{s}}\right), c\left(\mu^{-1},\left(\lambda_{1} \ldots \lambda_{n}\right)^{-k}\right)=k \ell_{\beta}\left(\lambda_{1} \ldots \lambda_{n}\right)$ for any positive number $k$. 
In particular, we just proved the following result.

THEOREM 12.6. If $G$ is the coordinate group of a quadratic equation, then there exists a free regular Lyndon length function $\ell: G \rightarrow \mathbb{Z}^{2}$, where $\mathbb{Z}^{2}$ is ordered lexicographically.

Two elements $x$ and $y$ in a group $G$ with the length function $\ell$ are said to be commensurable if there exist natural numbers $m$ and $n$ such that $m \ell(x) \geq \ell(y)$ and $n \ell(y) \geq \ell(x)$.

Notice, that for all possible solutions $\beta$ in general position with respect to $Q$ and coordinate groups $F_{R\left(S_{\beta}\right)}$ there is a finite number of possible generalized equations in the overlapping form up to the values of constant bases.

Let now $\mathcal{Q}=\left\{Q_{1}, \ldots, Q_{k}\right\}$ be a family of all the MQH vertex groups in the JSJ decomposition of $G$ (modulo subgroups $K_{1}, \ldots K_{s}$ ). For each solution $\beta: G \rightarrow F$ we can construct a group $F_{R\left(S_{\beta}\right)}$ which is the coordinate group of a quadratic system corresponding to $\mathcal{Q}$ over $F$. Denote this system $\mathcal{Q}^{\prime}$. Starting with a positive unbounded family of solutions corresponding to $\mathcal{Q}^{\prime}$ we can similarly construct a length function $\ell_{\beta}: F_{R\left(S_{\beta}\right)} \rightarrow Z^{2}$.

Let $\mathcal{A}=\left\{A_{1}, \ldots, A_{l}\right\}$ be a family of all the abelian vertex groups in the JSJ decomposition of $G$ (modulo subgroups $K_{1}, \ldots K_{s}$ ). For each group $F_{R\left(S_{\beta}\right)}$ where the images of the edge groups adjacent to subgroups from $\mathcal{A}$ are non-trivial, we can assign a group $F_{R\left(P_{\beta}\right)}$ which is obtained by an extension of centralizers of these images by the groups from $\mathcal{A}$. Denote this family in $F_{R\left(P_{\beta}\right)}$ by $\mathcal{A}^{\prime}$. If $m$ is the maximum of ranks of the groups from $\mathcal{A}^{\prime}$, then one can construct a free regular Lyndon's length function $L_{\beta}: F_{R\left(P_{\beta}\right)} \rightarrow Z^{m}$.

12.5. Free regular Lyndon length functions on NTQ groups. In this section we construct regular free length functions with values in $\mathbb{Z}^{n}$ on a coordinate group of an NTQ system.

THEOREM 12.7. If $G$ is the coordinate group of an NTQ system, then there exists a free regular Lyndon length function $\ell: G \rightarrow \mathbb{Z}^{t}$, where $\mathbb{Z}^{t}$ is ordered lexicographically.

Proof. Consider a NTQ system $\mathcal{S}$ :

$$
\begin{gathered}
S_{1}\left(X_{1}, \ldots, X_{n}\right)=1, \\
\ldots \\
S_{n}\left(X_{n}\right)=1 .
\end{gathered}
$$

Construct a solution $X=X_{1} \cup \ldots \cup X_{n}$ inductively. If $S_{n}=1$ is a regular quadratic equation, we denote $\Theta_{n}=\phi \beta$, where $\beta$ is some solution in a general position and $\phi$ belongs to a positive unbounded family of automorphisms for $F_{R\left(S_{n}\right)}$. If $S_{n}=1$ is a system of the type $U_{\text {com }}$ (see Definition 39 in [40]), and $U_{\text {com }}:\left\{\left[y_{p}, y_{s}\right]=\right.$ $\left.1,\left[y_{p}, u\right]=1, p, s=1, \ldots, r\right\}$, we take a solution $y_{1}=u^{N}, \ldots, y_{r}=u^{N^{r}}$ and also denote it by $\theta_{n}$. Now, if $S_{i}=1$ is a regular quadratic equation, we denote $\Theta_{i}=\phi \Theta_{i+1}$, where $\phi$ belongs to a positive unbounded family of automorphisms. If $S_{i}=1$ is a system of the type $U_{c o m}:\left\{\left[y_{p}, y_{s}\right]=1,\left[y_{p}, u^{\theta_{i+1}}\right]=1, p, s=1, \ldots, r\right\}$, we take a solution $y_{1}=u^{N \theta_{i+1}}, \ldots, y_{r}=u^{N^{r} \theta_{i+1}}$ and also denote it by $\theta_{i}$. Finally, $\theta_{1}$ is a solution for $X$. A family of solutions $\Theta=\Theta_{1}$ is called positive unbounded family of solutions for the NTQ system. One can construct a generalized equation for a 
positive unbounded family of solutions on $F_{R(S)}$ and then by induction construct a regular length function $\ell: F_{R(S)} \rightarrow Z^{t}$.

Every positive unbounded family of solutions $\Theta$ is a generic family of solutions for $\mathcal{S}$. We recall the definition of a generic family of solutions of a NTQ system.

Definition 12.8. A family of solutions $\Theta$ of a regular quadratic equation $S(X)=1$ over a group $G$ is called generic if for any equation $V(X, Y, A)=1$ with coefficients in $G$ the following is true: if for any solution $\theta \in \Theta$ there exists a solution of $V\left(X^{\theta}, Y, A\right)=1$ in $G$, then $V=1$ admits a complete $S$-lift. If the equation $S(X)=1$ is empty $\left(G_{R(S)}=G * F(X)\right)$ we always take as a generic family a sequence of growing different Merzlyakov's words (defined in [40, Section 4.4).

Let $W(X, A)=1$ be a NTQ system that consists of equations $S_{1}\left(X_{1}, \ldots, X_{n}\right)=$ $1, \ldots, S_{n}\left(X_{n}\right)=1$. A family of solutions $\Psi$ of $W(X, A)=1$ is called generic if $\Psi=\Psi_{1} \ldots \Psi_{n}$, where $\Psi_{i}$ is a generic family of solutions of $S_{i}=1$ over $G_{i+1}$ if $S_{i}=1$ is a regular quadratic system, and $\Psi_{i}$ is a discriminating family for $S_{i}=1$ if it is a system of the type $U_{c o m}$.

\section{Effective construction of JSJ decompositions of groups from $\mathcal{F}$.}

In this section we prove the following result.

THEOREM 13.1. There exists an algorithm to obtain a cyclic [abelian] JSJ decomposition for a f.g. fully residually free group $G$ modulo a given finite family of finitely generated subgroups. The algorithm constructs a presentation of $G$ as a fundamental group of a JSJ decomposition.

13.1. Construction of $T_{J S J}$. In this subsection we will extend the tree $T(\Omega)$ to a tree $T_{\text {ext }}(\Omega)$ which will serve as an auxiliary tree for the construction of the tree $T_{J S J}$. This tree will be used to construct a JSJ decomposition of the group $G$.

We are looking for a JSJ decomposition modulo subgroups $F \leq K_{1}, \ldots, K_{s}$ of $G$. Let $K_{i}$ be generated by $h_{i 1}, \ldots, h_{i j_{i}}$. We represent $h_{i k}=g_{i}^{-1} \bar{h}_{i k} g_{i}$ for $k=$ $2, \ldots, j_{i}$ and put all the elements $h_{1 k}, k=1, \ldots, i_{1}^{\prime}, \bar{h}_{i k}, i=1, \ldots s, k=1, \ldots, j_{i}$ into the parametric parts of the initial generalized equations. We include the case when some of $g_{i}$ 's are trivial.

We do not consider in the construction of $T_{\text {ext }}(\Omega)$ vertices of type 1 as final vertices. In other words, at each vertex $v$ of type 1 we glue a tree $T\left(\Omega_{v}\right)$ and iterate this process. We introduce also a new case 12.1, which can have place together with case 12 and produces an auxiliary edge.

Case 12.1 Announce all the quadratic closed sections to be non-active sections. The corresponding edge is auxiliary.

Proposition 13.2. Let $\mathcal{Q}=\left\{Q_{1}, \ldots, Q_{s}\right\}$ and $\mathcal{A}=\left\{A_{1}, \ldots, A_{l}\right\}$ be respectively all MQH subgroups and all abelian vertex groups in an abelian JSJ decomposition of a freely indecomposable fully residually free group $G$ (modulo subgroups $\left.K_{1}, \ldots, K_{s}\right)$. There exists a generalized equation $\Omega$ for $G$ such that a discriminating family of $F$-solutions of $U=1$ onto different groups $F_{R\left(P_{\beta}\right)}$ that take subgroups from $\mathcal{Q}$ and $\mathcal{A}$ onto their images in $\mathcal{Q}^{\prime}$ and $\mathcal{A}^{\prime}$ factors through $\Omega$. Moreover, one can effectively construct a finite subtree $T_{J S J}(\Omega)$ of $T_{\text {ext }}(\Omega)$ with the property that such a family of solutions factors through $\Omega_{v}$ for a leaf vertex $v$ (of type 2) of $T_{J S J}(\Omega)$. 
Proof. When we consider a solution $\bar{H}$ of the generalized equation $\Omega$, it is a solution in some group $F_{R\left(P_{\beta}\right)}$ which has a regular free length function $L_{\beta}$. Since we consider here an arbitrary solution from the family, we denote by $\ell$ the length function $L_{\beta}$ which corresponds to the given solution. All these length functions are similar.

If we have an infinite path in $T(\Omega)$

$$
v_{1} \rightarrow v_{2} \rightarrow \ldots v_{r} \rightarrow \ldots,
$$

then by Lemma 5.5 we have three possibilities: 1) a linear case, 2) a quadratic case, 3) case 15 .

1) In this case $G$ must be conjugated into the coordinate group of the kernel of $F_{R\left(\Omega_{v_{n}}\right)}$, and we can work with the kernel of $\Omega_{v_{n}}$ instead of it.

2) In this case there is an auxiliary edge. Transformation of Case 12.1 and 15.1 decreases the complexity $\tau^{\prime}$.

3) Since there is only a finite family of generalized equations for $U=1$, there exists at least one of them such that a discriminating family of solutions onto groups $F_{R\left(P_{\beta}\right)}$ that are retracts on $F$ and map $\mathcal{Q}, \mathcal{A}$ onto $\mathcal{Q}^{\prime}, \mathcal{A}^{\prime}$ factors through $\Omega$. Denote this family by $\Psi$. Let $\mathcal{Q}_{\text {inf }}$ be a family of $Q H$ vertex groups $Q$ of $F_{R(\Omega)}$ such that $Q^{g} \cap H$ has infinite index in $Q^{g}$ for any $g \in F_{R(\Omega)}$. We take for each solution in $\Psi$ an equivalent solution minimal with respect to groups of automorphisms of $\mathcal{Q}_{\text {inf }}$ among solutions that extend to a solution of $\Omega$.

Denote by $\mathcal{Q}_{\text {fin }}$ the set of $\mathrm{QH}$ vertex groups $Q$ of $F_{R(\Omega)}$ such that for some $g \in F_{R(\Omega)}$, the intersection $Q^{g} \cap G$ has a finite index in $Q^{g}$. Let a quadratic section $\left[j_{1}, j_{2}\right]$ of $\tilde{\Omega}$ (see transformation D3) correspond to a $\mathrm{QH}$ subgroup conjugated into one of the subgroups in $\mathcal{Q}_{\text {fin }}$.

Let $\bar{H}$ be a solution of $\Omega$. Denote by $d_{1}(\bar{H})$ the length of $\bar{H}\left[j_{1}, j_{2}\right]$ and by $d_{2}(\bar{H})$ the sum of the lengths of the quadratic-coefficient bases on $\left[j_{1}, j_{2}\right]$. If $d_{2}(\bar{H}) \geq(r, 1)$ for some $r \in Z$, then there exists a solution $\bar{H}^{+}$of $\Omega$ equivalent to $\bar{H}$ with respect to the group of automorphisms of $Q \cap H$ such that

$$
d_{1}(\bar{H}) \leq f_{1}(\Omega) d_{2}(\bar{H})
$$

where $f_{1}(\Omega)$ is a function from Lemma 32. If $d_{2}(\bar{H})=(r, 0)$ for some $r \in Z$, then there exists a solution $\bar{H}^{+}$of $\Omega$ equivalent to $\bar{H}$ with respect to the group of automorphisms of $Q \cap H$ such that

$$
d_{1}(\bar{H}) \leq f_{1}(\Omega)(0,1),
$$

where $f_{1}(\Omega)$ is a function similar to the one from Lemma 32, maybe slightly increased.

For quadratic sections corresponding to $\mathcal{Q}_{\text {fin }}$ we replace $\bar{H}$ by $\bar{H}^{+}$. Denote the new family of solutions of $U=1$ by $\Psi_{\min }$. We will show that every path in $T(\Omega)$ for a family $\Psi_{\min }$ after finite number of steps either does not have active bases or comes to a vertex $v$ such that $\Omega_{v}$ begins with an overlapping pair of bases, and, therefore, has an outgoing auxiliary edge.

LEMMA 13.3. Suppose for the subinterval $[1, \delta]$ of the interval I of a generalized equation $\Omega$ and a solution $\bar{H}$ of $\Omega$ we have $d_{1}(\bar{H}[1, \delta]) \leq f_{1}(\Omega) d_{2}(\bar{H}[1, \delta])$. Let $\Omega=\Omega_{v_{1}}$ and there is a path

$$
r=v_{1} \rightarrow v_{2} \rightarrow \ldots \rightarrow v_{m},
$$


where $\operatorname{tp}\left(v_{i}\right)=15(1 \leq i \leq m)$ in the tree $T(\Omega)$, the leading base of $\Omega_{v_{i}}(1 \leq i \leq m)$ does not overlap with its double, and the initial boundary of $\Omega_{v_{m}}$ is to the left of the boundary $\delta$ of $\Omega$. Then this path cannot contain a prohibited subpath.

Proof. The proof repeats the proof of Proposition 7.5 for a generalized equation in a group with non-archimedian length function. We consider a subpath (45) corresponding to the fragment

$$
\left(\Omega_{v_{1}}, \bar{H}^{(1)}\right) \rightarrow\left(\Omega_{v_{2}}, \bar{H}^{(2)}\right) \rightarrow \ldots \rightarrow\left(\Omega_{v_{m}}, \bar{H}^{(m)}\right) \rightarrow \ldots
$$

of the sequence (47). Here $v_{1}, v_{2}, \ldots, v_{m-1}$ are vertices of the tree $T_{0}(\Omega)$, and for all vertices $v_{i}$ the edge $v_{i} \rightarrow v_{i+1}$ is principal.

Let $\mu_{i}$ denote the carrier base of $\Omega_{v_{i}}$, and $\omega=\left\{\mu_{1}, \ldots, \mu_{m-1}\right\}$, and $\tilde{\omega}$ denote the set of such bases which are transfer bases for at least one equation in (86). By $\omega_{1}$ denote the set of such bases $\mu$ for which either $\mu$ or $\Delta(\mu)$ belongs to $\omega \cup \tilde{\omega}$; by $\omega_{2}$ denote the set of all the other bases. Let

$$
\alpha(\omega)=\min \left(\min _{\mu \in \omega_{2}} \alpha(\mu), \delta\right) .
$$

Let $X_{\mu} \doteq H[\alpha(\mu), \beta(\mu)]$. If $(\Omega, \bar{H})$ is a member of sequence (86), then denote

$$
\begin{gathered}
d_{\omega}(\bar{H})=\sum_{i=1}^{\alpha(\omega)-1} \ell\left(H_{i}\right), \\
\psi_{\omega}(\bar{H})=\sum_{\mu \in \omega_{1}} \ell\left(X_{\mu}\right)-2 d_{\omega}(\bar{H}) .
\end{gathered}
$$

Every item $h_{i}$ of the section $[1, \alpha(\omega)]$ belongs to at least two bases, and both bases are in $\omega_{1}$, hence $\psi_{\omega}(\bar{H}) \geq 0$.

Consider the quadratic part of $\tilde{\Omega}_{v_{1}}$ which is situated to the left of $\alpha(\omega)$. The solution $\bar{H}^{(1)}$ is minimal with respect to the canonical group of automorphisms corresponding to this vertex. We have

$$
d_{1}\left(\bar{H}^{(1)}\right) \leq f_{1}\left(\Omega_{v_{1}}\right) d_{2}\left(\bar{H}^{(1)}\right) .
$$

Using this inequality we estimate the length of the interval participating in the process $d_{\omega}\left(\bar{H}^{(1)}\right)$ from above by a product of $\psi_{\omega}$ and some function depending on $f_{1}$. This will be inequality (94). Then we will show that for a prohibited subpath the length of the participating interval must be reduced by more than this figure (equalities (100), (101)). This will imply that there is no prohibited subpath in the path (86).

Denote by $\gamma_{i}(\omega)$ the number of bases $\mu \in \omega_{1}$ containing $h_{i}$. Then

$$
\sum_{\mu \in \omega_{1}} \ell\left(X_{\mu}^{(1)}\right)=\sum_{i=1}^{\rho} \ell\left(H_{i}^{(1)}\right) \gamma_{i}(\omega),
$$

where $\rho=\rho\left(\Omega_{v_{1}}\right)$. Let $I=\left\{i \mid 1 \leq i \leq \alpha(\omega)-1 \& \gamma_{i}=2\right\}$ and $J=\{i \mid 1 \leq i \leq$ $\left.\alpha(\omega)-1 \& \gamma_{i}>2\right\}$. By [87]

$$
d_{\omega}\left(\bar{H}^{(1)}\right)=\sum_{i \in I} \ell\left(H_{i}^{(1)}\right)+\sum_{i \in J} \ell\left(H_{i}^{(1)}\right)=d_{1}\left(\bar{H}^{(1)}\right)+\sum_{i \in J} \ell\left(H_{i}^{(1)}\right) .
$$

Let $(\lambda, \Delta(\lambda))$ be a pair of quadratic-coefficient bases of the equation $\tilde{\Omega}_{v_{1}}$, where $\lambda$ belongs to the nonquadratic part. This pair can appear only from the bases $\mu \in \omega_{1}$. There are two types of quadratic-coefficient bases. 
Type 1. $\lambda$ is situated to the left of the boundary $\alpha(\omega)$. Then $\lambda$ is formed by items $\left\{h_{i} \mid i \in J\right\}$ and hence $\ell\left(X_{\lambda}\right) \leq \sum_{i \in J} \ell\left(H_{i}^{(1)}\right)$. Thus the sum of the lengths $\ell\left(X_{\lambda}\right)+\ell\left(X_{\Delta(\lambda)}\right)$ for quadratic-coefficient bases of this type is not more than $2 n \sum_{i \in J} \ell\left(H_{i}^{(1)}\right)$.

Type 2. $\lambda$ is situated to the right of the boundary $\alpha(\omega)$. The sum of length of the quadratic-coefficient bases of the second type is not more than $2 \sum_{i=\alpha(\omega)}^{\rho} \ell\left(H_{i}^{(1)}\right) \gamma_{i}(\omega)$.

We have

$$
d_{2}\left(\bar{H}^{(1)}\right) \leq 2 n \sum_{i \in J} \ell\left(H_{i}^{(1)}\right)+2 \sum_{i=\alpha(\omega)}^{\rho} \ell\left(H_{i}^{(1)}\right) \gamma_{i}(\omega) .
$$

Now (88) and (90) imply

$$
\psi_{\omega}\left(\bar{H}_{i}^{(1)}\right) \geq \sum_{i \in J} \ell\left(H_{i}^{(1)}\right)+\sum_{i=\alpha(\omega)}^{\rho} \ell\left(H_{i}^{(1)}\right) \gamma_{i}(\omega) .
$$

Inequalities (89), (91), (92), (93) imply

$$
d_{\omega}\left(\bar{H}^{(1)}\right) \leq \max \left\{\psi_{\omega}\left(\bar{H}^{(1)}\right)\left(2 n f_{1}\left(\Omega_{v_{1}}\right)+1\right), f_{1}\left(\Omega_{v_{1}}\right)\right\}
$$

From the definition of Case 15 it follows that all the words $H^{(i)}\left[1, \rho_{i}+1\right]$ are the ends of the word $H^{(1)}\left[1, \rho_{1}+1\right]$, that is

$$
H^{(1)}\left[1, \rho_{1}+1\right] \doteq U_{i} H^{(i)}\left[1, \rho_{i}+1\right] .
$$

On the other hand bases $\mu \in \omega_{2}$ participate in these transformations neither as carrier bases nor as transfer bases; hence $H^{(1)}\left[\alpha(\omega), \rho_{1}+1\right]$ is the end of the word $H^{(i)}\left[1, \rho_{i}+1\right]$, that is

$$
H^{(i)}\left[1, \rho_{i}+1\right] \doteq V_{i} H^{(1)}\left[\alpha(\omega), \rho_{1}+1\right] .
$$

So we have

$$
d_{\omega}\left(\bar{H}^{(i)}\right)-d_{\omega}\left(\bar{H}^{(i+1)}\right)=\ell\left(V_{i}\right)-\ell\left(V_{i+1}\right)=\ell\left(U_{i+1}\right)-\ell\left(U_{i}\right)=\ell\left(X_{\mu_{i}}^{(i)}\right)-\ell\left(X_{\mu_{i}}^{(i+1)}\right) .
$$

In particular (51), (97) imply that $\psi_{\omega}\left(\bar{H}^{(1)}\right)=\psi_{\omega}\left(\bar{H}^{(2)}\right)=\ldots \psi_{\omega}\left(\bar{H}^{(m)}\right)=\psi_{\omega}$. Denote the number (97) by $\delta_{i}$.

Let the path (45) be $\mu$-reducing, that is either $\mu_{1}=\mu$ and $v_{2}$ does not have auxiliary edges and $\mu$ occurs in the sequence $\mu_{1}, \ldots, \mu_{m-1}$ at least twice, or $v_{2}$ does have auxiliary edges $v_{2} \rightarrow w_{1}, \ldots v_{2} \rightarrow w_{k}$ and the base $\mu$ occurs in the sequence $\mu_{1}, \ldots, \mu_{m-1}$ at least $\max _{1 \leq i \leq k} s\left(\Omega_{w_{i}}\right)$ times. Estimate $\ell\left(U_{m}\right)=\sum_{i=1}^{m-1} \delta_{i}$ from below. First notice that if $\mu_{i_{1}}=\mu_{i_{2}}=\mu\left(i_{1}<i_{2}\right)$ and $\mu_{i} \neq \mu$ for $i_{1}<i<i_{2}$, then

$$
\sum_{i=i_{1}}^{i_{2}-1} \delta_{i} \geq \ell\left(H^{i_{1}+1}\left[1, \alpha\left(\Delta\left(\mu_{i_{1}+1}\right)\right)\right]\right) .
$$

Indeed, if $i_{2}=i_{1}+1$, then $\delta_{i_{1}}=\ell\left(H^{\left(i_{1}\right)}[1, \alpha(\Delta(\mu))]=\ell\left(H^{\left(i_{1}+1\right)}[1, \alpha(\Delta(\mu))]\right.\right.$. If $i_{2}>i_{1}+1$, then $\mu_{i_{1}+1} \neq \mu$ and $\mu$ is a transfer base in the equation $\Omega_{v_{i_{1}+1}}$. Hence $\delta_{i_{1}+1}+\ell\left(H^{\left(i_{1}+2\right)}[1, \alpha(\mu)]\right)=\ell\left(H^{\left(i_{1}+1\right)}\left[1, \alpha\left(\mu_{i_{1}+1}\right)\right]\right)$. Now (98) follows from

$$
\sum_{i=i_{1}+2}^{i_{2}-1} \delta_{i} \geq \ell\left(H^{\left(i_{1}+2\right)}[1, \alpha(\mu)]\right)
$$


So if $v_{2}$ does not have outgoing auxiliary edges, that is the bases $\mu_{2}$ and $\Delta\left(\mu_{2}\right)$ do not intersect in the equation $\Omega_{v_{2}}$; then (98) implies that

$$
\sum_{i=1}^{m-1} \delta_{i} \geq \ell\left(H^{(2)}\left[1, \alpha\left(\Delta \mu_{2}\right)\right]\right) \geq \ell\left(X_{\mu_{2}}^{(2)}\right) \geq \ell\left(X_{\mu}^{(2)}\right)=\ell\left(X_{\mu}^{(1)}\right)-\delta_{1},
$$

which implies that

$$
\sum_{i=1}^{m-1} \delta_{i} \geq \frac{1}{2} \ell\left(X_{\mu}^{(1)}\right)
$$

Suppose now that the path (45) is prohibited; hence it can be represented in the form (46). From definition (88) we have $\sum_{\mu \in \omega_{1}} \ell\left(X_{\mu}^{(m)}\right) \geq \psi_{\omega}$; so at least for one base $\mu \in \omega_{1}$ the inequality $\ell\left(X_{\mu}^{(m)}\right) \geq \frac{1}{2 n} \psi_{\omega}$ holds. Because $X_{\mu}^{(m)} \doteq\left(X_{\Delta(\mu)}^{(m)}\right)^{ \pm 1}$, we can suppose that $\mu \in \omega \cup \tilde{\omega}$. Let $m_{1}$ be the length of the path $r_{1} s_{1} \ldots r_{l} s_{l}$ in (46). If $\mu \in \tilde{\omega}$ then by the third part of the definition of a prohibited path there exists $m_{1} \leq i \leq m$ such that $\mu$ is a transfer base of $\Omega_{v_{i}}$. Hence, $\ell\left(X_{\mu_{i}}^{\left(m_{1}\right)}\right) \geq \ell\left(X_{\mu_{i}}^{(i)}\right) \geq$ $\ell\left(X_{\mu}^{(i)}\right) \geq \ell\left(X_{\mu}^{(m)}\right) \geq \frac{1}{2 n} \psi_{\omega}$. If $\mu \in \omega$, then take $\mu$ instead of $\mu_{i}$. We proved the existence of a base $\mu \in \omega$ such that

$$
\ell\left(X_{\mu}^{\left(m_{1}\right)}\right) \geq \frac{1}{2 n} \psi_{\omega}
$$

By the definition of a prohibited path, the inequality $\ell\left(X_{\mu}^{(i)}\right) \geq \ell\left(X_{\mu}^{\left(m_{1}\right)}\right)(1 \leq i \leq$ $m_{1}$ ), (99), and (100) we obtain

$$
\sum_{i=1}^{m_{1}-1} \delta_{i} \geq \max \left\{\frac{1}{20 n} \psi_{\omega}, 1\right\}\left(40 n^{2} f_{1}+20 n+1\right) .
$$

By (97) the sum in the left part of the inequality (101) equals $d_{\omega}\left(\bar{H}^{(1)}\right)-$ $d_{\omega}\left(\bar{H}^{\left(m_{1}\right)}\right)$; hence

$$
d_{\omega}\left(\bar{H}^{(1)}\right) \geq \max \left\{\frac{1}{20 n} \psi_{\omega}, 1\right\}\left(40 n^{2} f_{1}+20 n+1\right),
$$

which contradicts to (94).

This contradiction was obtained from the supposition that there are prohibited paths (86) in the path (47). Hence (47) does not contain prohibited paths.

By Lemma 13.3 in the case of inequality (833) we cannot have a prohibited subpath.

Suppose we have inequality (84) and the total length of the section $[1, \alpha(\omega)]$ is greater than $(s, 1)$ for some $s \in Z$. The only infinite path we can have is path (85). Instead of inequality (57) we now have

$$
d_{\omega}\left(\bar{H}^{(1)}\right) \leq(0,1) 2 n\left(f_{1}\left(\Omega_{v_{1}}\right)+1\right) .
$$

For a $\mu$-reducing path we still have inequality (62), and, automatically (66). In the absence of overlapping pair bases there exists a base $\mu \in \omega$ such that

$$
\ell\left(X_{\mu}^{\left(m_{1}\right)}\right) \geq(r, 1)
$$

for some $r \in Z$. By the definition of the prohibited subpath, we obtain

$$
d_{\omega}\left(\bar{H}^{(1)}\right)-d_{\omega}\left(\bar{H}^{\left(m_{1}\right)}\right)=\sum_{i=1}^{m_{1}-1} \delta_{i} \geq(1 / 10)(r, 1)\left(40 n^{2} f_{1}+20 n+1\right)
$$


which contradicts to (102). The Lemma is proved.

The proposition is proved.

13.2. Periodic structures and overlapping pair sections. Leaf vertices of $T_{J S J}$ have type 2 . We now replace each length function $L_{\beta}: F_{R\left(P_{\beta}\right)} \rightarrow \mathbb{Z}^{m}$ by the function $\ell_{\beta}: F_{R\left(S_{\beta}\right)} \rightarrow \mathbb{Z}^{2}$ by specializing elements from $\mathcal{A}$. Namely, for each abelian group $A_{i}$ instead of a homomorphism onto $A_{i}^{\prime}$ we take a positive unbounded family of homomorphisms onto $F$. Therefore, solutions of generalized equations at final vertices of $T_{J S J}$ will be now considered as solutions in $F_{R\left(S_{\beta}\right)}$. Like in the previous subsection, if $H$ is a solution of a generalized equation in $F_{R\left(S_{\beta}\right)}$, then by $\ell\left(H_{i}\right)$ we denote $\ell_{\beta}\left(H_{i}\right)$.

Definition 13.4. A triple of elements $\left(A, T, A^{\prime}\right)$ of $F_{R\left(S_{\beta}\right)}$ forms an overlapping pair section for $S_{\beta}=1$ if a quadratic generalized equation corresponding to a quadratic equation $S_{\beta}=1$ forms a closed section $A \circ T=T \circ A^{\prime}$, two occurrences of $T$ in this equation overlap, and $A$ is not a proper power.

A generalized equation $\Omega$ at a vertex of type 2 can be written as a union of closed sections each having a principal overlapping pair of bases.

Lemma 13.5. If $\left(B, T, B^{\prime}\right)$ is an overlapping pair section for a (regular) quadratic equation $S_{\beta}=1$, then

(1) for any $U, V \in F_{R\left(S_{\beta}\right)}$ such that $T=U \circ V$, either $V$ infinitely smaller $(<<)$ than $T$ or $U<<T$,

(2) In the case $U<<T, U=B^{n}$.

Definition 13.6. A solution $H$ of a generalized equation $\Omega$ is called periodic with respect to a period $P$, where $P$ is not a proper power, if for every non-parametric closed section $[i, j]$ containing at least one base the word $H[i, j]$ can be represented in one of the following forms:

1)

$$
H[i, j]=A^{r} A_{1}
$$

( $r \geq 2, A=A_{1} A_{2}, A$ is a primitive element.)

2) $H[i, j]=A^{r} T A_{1}=T A^{\prime r} A_{1}$, where $\left(A, T, A^{\prime}\right)$ forms an overlapping pair section for some $S_{\beta}=1$, with $T$ infinitely larger than $A$ and $c\left(T, A^{n}\right)=\ell\left(A^{n}\right)$, $r \in \mathbb{Z}$, and $A^{\prime}=A_{1} \circ A_{2}$.

In addition, it is possible to define a function $\ell_{1}$ in the definition of $\ell_{\beta}$ such a way that $\ell_{\beta}(A) \leq \ell_{\beta}(P)$ and for at least one such closed section $[i, j]$ the element $A$ must be a cyclic shift of the element $P^{ \pm 1}$.

In our new situation Lemmas 6.10 6.14 still hold. Instead of Lemma 6.15 we have the following

Lemma 13.7. Suppose that the generalized equation $\Omega$ is periodized with respect to a non-singular periodic structure $\mathcal{P}$. Then for any periodic solution $H$ of $\Omega$ we can choose a tree BT, some set of variables $S=\left\{h_{j_{1}}, \ldots, h_{j_{s}}\right\}$ and a solution $H^{+}$of $\Omega$ equivalent to $H$ with respect to the group of canonical transformations $\bar{A}(\Omega)$ such a way that each of the bases $\lambda_{i} \in B T \backslash B T_{0}$ can be represented as $\lambda_{i}=\lambda_{i 1} h_{k_{i}} \lambda_{i 2}$, where $h_{k_{i}} \in S$ and for any $h_{j} \in S, \ell\left(H_{j}^{+}\right) \leq f_{3} \ell(P)$, where $f_{3}$ is some constructible function depending on $\Omega$. This representation gives a new generalized equation $\Omega^{\prime}$ periodic with respect to a periodic structure $\mathcal{P}^{\prime}$ with the same period $P$ and all $h_{j} \in S$ 
considered as variables not from $\mathcal{P}^{\prime}$. The graph $B \Gamma^{\prime}$ for the periodic structure $\mathcal{P}^{\prime}$ has the same set of vertices as $B \Gamma$, has empty set $C^{(2)}$ and either $B T^{\prime}=B T_{0}^{\prime}$ or the difference between $B T^{\prime}$ and $B T_{0}^{\prime}$ consists of one infinitely large edge.

Let $c$ be a cycle from $C^{(1)}$ of minimal length, $\ell(c)=n_{c} \ell(P)$. Using canonical automorphisms from $A(\Omega)$ one can transform any solution $H$ of $\Omega$ into a solution $H^{+}$such that for any $h_{j} \in S, \ell\left(H_{j}^{+}\right) \leq f_{3} \ell(c)$. Let $\mathcal{P}^{\prime}$ be a periodic structure, in which all $h_{i} \in S$ are considered as variables not from $\mathcal{P}^{\prime}$, then $B \Gamma^{\prime}$ has empty set $C^{(2)}$ and either $B T^{\prime}=B T_{0}^{\prime}$ or the difference between $B T^{\prime}$ and $B T_{0}^{\prime}$ consists of one infinitely large edge.

The proof is the same as the proof of Lemma 6.15

Notice that if a solution $H$ of a generalized equation is periodic with respect to some period $P$, and $\left(B, T, B^{\prime}\right)$ forms an overlapping pair section for some $S_{\beta}=$ 1, with $T$ infinitely larger than $B$, then either $B$ is a cyclic shift of $P$ or the length function $\ell_{1}$ in the definition of $\ell_{\beta}$ can be redefined such a way that $\ell_{\beta}(P) \leq$ $\ell_{\beta}(B)$. Therefore this lemma can be applied to each closed section that contains an infinitely long overlapping pair of bases. Notice also that for any periodic structure $\mathcal{P}$ the statements of Lemmas 6.10 6.11 are true because all the other periodic structures can be temporary considered as parameters.

13.3. Recognizing $\mathbf{Q H}$ and abelian subgroups using $T_{J S J}$. Let $v$ be a leaf vertex of $T_{J S J}$ such that a discriminating family of solutions of $U=1$ onto groups $F_{R\left(S_{\beta}\right)}$ factors through $\Omega_{v}$. A closed section from $\Omega_{v}$ either belongs to some periodic structure or can be considered as an overlapping form for some quadratic generalized equation $\Omega_{\text {quadr }}$ with some extra bases situated on it. For each such overlapping pair section

$$
\nu_{1} \ldots \nu_{s} \Delta(\mu)=\mu \theta_{1} \ldots \theta_{k}
$$

replace this section by two closed sections $\lambda_{2} \Delta\left(\mu_{1}\right)=\mu_{1} \Delta\left(\lambda_{2}\right)$ and $\lambda_{3} \Delta\left(\mu_{2}\right)=$ $\mu_{2} \Delta\left(\lambda_{3}\right)$. Put all the other bases from the left part of the overlapping pair section (105) to $\lambda_{2} \Delta\left(\mu_{1}\right)=\mu_{1} \Delta\left(\lambda_{2}\right)$ and all the other bases from the right part of the overlapping pair section (105) to $\lambda_{3} \Delta\left(\mu_{2}\right)=\mu_{2} \Delta\left(\lambda_{3}\right)$. Keep also the basic equations $\nu_{i}=\Delta\left(\nu_{i}\right)$, where $\alpha\left(\nu_{1}\right)=\alpha\left(\lambda_{2}\right), \beta\left(\nu_{s}\right)=\beta\left(\lambda_{2}\right)$ and $\theta_{i}=\Delta\left(\theta_{i}\right)$, where $\alpha\left(\theta_{1}\right)=$ $\alpha\left(\lambda_{3}\right), \beta\left(\theta_{k}\right)=\beta\left(\lambda_{3}\right)$. Let $X_{\mu_{1}}$ and $X_{\mu_{2}}$ have minimal necessary lengths (defined by Lemma 13.7] to accommodate all the extra bases for a minimal solution. Apply the entire transformation to this new generalized equation. After a bounded number of steps all the extra bases will be situated on the bases that where quadraticcoefficient bases of generalized equations $\Omega_{q u a d r}$. Denote these new generalized equations by $\Omega_{\text {quadr }}^{\prime}$. Replace in the generalized equation $\Omega_{v}$ the closed sections that we have changed and which were denoted by $\Omega_{\text {quadr }}$ by the generalized equations $\Omega_{\text {quadr }}^{\prime}$. Denote this generalized equation by $\Omega_{v}^{\prime}$.

Proposition 13.8. There is an algorithm to find all $M Q H$ and abelian vertex groups in a JSJ decomposition of $G$ (modulo subgroups $\left.K_{1}, \ldots, K_{s}\right)$.

Proof. If $F_{R\left(\Omega_{v}^{\prime}\right)}$ is a free product, then $G$ is conjugated into a factor in this free decomposition. We can consider all possible periodic structures corresponding to each vertex $v$ of type 2. All MQH subgroups of $G$ can be obtained as $G \cap Q^{g}$, where $Q$ is a $\mathrm{QH}$ subgroup of $F_{R\left(\Omega_{v}^{\prime}\right)}$ for some $v$ and $\Omega_{v}^{\prime}$ and $G \cap Q^{g}$ has finite index in $Q^{g}$. Then this subgroup $Q^{g}$ belongs to the factor in a free decomposition 
of $F_{R\left(\Omega_{v}^{\prime}\right)}$ and we will consider this factor $K=F_{R(\mathcal{T})}$ instead of $F_{R\left(\Omega_{v}^{\prime}\right)}$. By Lemma 3.13 all intersections of conjugates of two subgroups can be found effectively.

Lemma 13.9. Let $\mathcal{Q}$ be a family of $M Q H$ vertex subgroups of a freely indecomposable fully residually free group $G$ (modulo subgroups $K_{1}, \ldots, K_{s}$ ) Then each $Q \in \mathcal{Q}$ is conjugated to one of the $Q H$ subgroups for $K$.

Proof. For a discriminating family of solutions $\Psi$ of $U=1$ which is positive unbounded with respect to $\mathcal{Q}$ equation $\mathcal{T}$ has a solution. A discriminating subfamily $\Psi_{1}$ of $\Psi$ which is also positive unbounded with respect to $\mathcal{Q}$ factors through one of the systems $U_{i}=1$ from Theorem 9.3 . By the Implicit function theorem (40], Theorem 12, see also 45, Theorem 10) $\mathcal{T}$ has a solution which takes subgroups from $\mathcal{Q}$ to subgroups from $\mathcal{Q}^{\prime}$ in some group obtained from $F_{R\left(U_{i}\right)}$ by adding roots of a finite number of elements from abelian vertex groups and by extending $F_{R\left(\bar{U}_{i}\right)}$ by some group without sufficient splitting. Therefore elements of QH subgroups of $K$ that have QH subgroups of $G$ as finite index subgroups actually coincide with these QH subgroups of $G$, and $Q=Q_{i}^{g}$.

We constructed $\Omega_{v}$ for a discriminated family of solutions of $U=1$ and length functions $L_{\beta}$, the infinitely long variables cannot disappear in the process of construction of $T_{J S J}$, therefore each abelian vertex group of $G$ will be seen in some periodic structure of $F_{R\left(\Omega^{\prime}\right)}$. Suppose an abelian group is given by relations $\left[y_{i}, y_{j}\right]=\left[y_{i}, u\right]=1, i, j=1, \ldots, r$. After we replaced length function $L_{\beta}$ by $\ell_{\beta}$ we have a solution $y_{1}=u^{N}, \ldots, y_{r}=u^{N^{r}}$ for an increasing sequence $\{N\}$. Therefore each abelian vertex group of $G$ is conjugated into an abelian vertex group of $F_{R\left(\Omega_{v}^{\prime}\right)}$ that corresponds to a periodic structure. If for a periodic structure the set of cycles $\bar{c}^{(2)}$ contains at least one cycle, then by Lemma 6.7 $\bar{c}^{(1)}$ and $\bar{c}^{(2)}$ generate an abelian vertex group of $F_{R\left(\Omega_{v}^{\prime}\right)}$, and each abelian vertex group of $G$ is conjugated into one of the abelian groups obtained this way. The proposition is proved.

13.4. Recognizing rigid subgroups. First we discuss how to construct a JSJ decomposition of $G$ modulo $F$. Let $D$ be a cyclic [abelian] such JSJ decomposition of $G=F_{R(U)}$. Consider a discriminating family of solutions for $U=1$ which for each edge group $G_{e}$ of $D$ contains infinitely increasing powers of the corresponding canonical Dehn' twists. If the edge group is non-cyclic abelian with generators $y_{1}, \ldots, y_{k}$, then we take infinitely increasing powers of the product of Dehn' twists corresponding to $y_{1}^{N}, \ldots, y_{k}^{N^{k}}$. Each solution from this family we pre-compose with a positive unbounded family of solutions for each MQH subgroup of $G$ and a family of solutions of the form $y_{1}=u^{N}, \ldots, y_{r}=u^{N^{r}}$ for an increasing sequence $\{N\}$ for each abelian vertex group of $G$ given by relations $\left[y_{i}, y_{j}\right]=\left[y_{i}, u\right]=1, i, j=1, \ldots, r$. All Dehn's twists that we consider fix elements from $F$. For different edges of $D$ corresponding canonical Dehn's twists commute, they also commute with canonical automorphisms corresponding to $\mathrm{MQH}$ and abelian vertex groups. Since all solutions of $U=1$ factor through a finite number of generalized equations, there is a generalized equation $\Omega$ such that a family of solutions with the properties described above factors through this generalized equation. In this case we will have a HNN splitting of $F_{R\left(\Omega_{v}^{\prime}\right)}$ and, therefore, a splitting of $K$ that induces the splitting of $G$ along $e$. We are able now to construct effectively an abelian JSJ decomposition for $G$. Considering different possibilities for periodic structures we have different decompositions for $F_{R\left(\Omega_{v}^{\prime}\right)}$. Each such decomposition consists of several abelian 
and $\mathrm{QH}$ vertex groups, one non-abelian non-QH vertex group, and some edges corresponding to HNN extensions. A JSJ decomposition $D$ of $G$ is induced by one of the decompositions of $F_{R\left(\Omega_{v}^{\prime}\right)}$, which we denote by $D\left(\Omega_{v}^{\prime}\right)$. In addition we already know all $\mathrm{QH}$ and abelian vertex groups for $D$. Denote by $S$ the non-abelian non-QH vertex group in the decomposition $D\left(\Omega_{v}^{\prime}\right)$, by $w_{S}$ the corresponding vertex and by $t_{1}, \ldots, t_{k}$ the stable letters corresponding to edges with initial and terminal vertex $w_{S}$. We know that each rigid subgroup in the JSJ decomposition of $G$ is the intersection of $G$ with $S^{t_{i_{1}} \ldots t_{i_{j}}}$ where the product $t_{i_{1}} \ldots t_{i_{j}}$ depends on the choice of the maximal subforest with rigid vertices and maximal subtree in the graph corresponding to the JSJ decomposition $D$ of $G$. Let $T$ be the Bass-Serre tree corresponding to $D\left(\Omega_{v}^{\prime}\right)$. We can effectively construct the induced decomposition of $G$ by Theorem 3.17 After we obtained induced abelian decompositions for different leaf vertices of $T_{J S J}$ and for different periodic structures associated with the same vertex, we can unfold them and we have to decide which of the induced decompositions is a JSJ decomposition of $G$. We can compare QH and abelian vertex groups for different induced decompositions and choose the ones with maximal family of such subgroups. We can transform all these decompositions into the form with the same QH and abelian vertex groups. If all the rigid vertex groups of such a decomposition are elliptic in all the other decompositions, then it is an abelian JSJ decomposition. Denote it by $D$. This decomposition is unique up to slidings, conjugations, and modifying boundary monomorphisms by conjugation. We can now collapse all edges with non-cyclic edge groups and obtain a cyclic JSJ decomposition.

Similarly we obtain an abelian JSJ decomposition modulo subgroups $K_{1}, \ldots, K_{s}$.

To construct a cyclic [abelian] JSJ decomposition $D$ of $G$ (not modulo $F$ ) we fix one of the rigid subgroups of $D$ (if exists) and begin with a discriminating family of solutions that contains increasing powers of Dehn's twists for all the edges of $D$, where all Dehn's twists fix elements of this subgroup. The rest of the argument is the same. In the case when $D$ does not have rigid subgroups, we begin with a discriminating family of solutions for $U=1$. Indeed, in this case all the edges have an abelian group as one of the vertex groups, and, therefore, they "will be seen" in some decomposition of some of the $F_{R\left(\Omega v^{\prime}\right)}$.

\section{Homomorphisms into NTQ groups}

The following result was proved in 41. It can be obtained by applying the elimination process to equations over an NTQ group (with regular free Lyndon length function) as described in Subsections 13.1 and 13.2

THEOREM 14.1. Let $G$ be a finitely generated group and $N$ an $N T Q$ group. Then:

(1) there exists a complete canonical $\operatorname{Hom}(G, N)$-diagram $\mathcal{C}$;

(2) if $N$ is a fixed subgroup of $G$ then there exists an $N$-complete canonical $\operatorname{Hom}_{N}(G, N)$-diagram $\mathcal{C}$.

Moreover, if the group $G$ is finitely presented then the Hom-diagrams from (1) and (2) can be found effectively.

This theorem implies the following result

Corollary 14.2. Let $G$ be a finitely generated group and $H$ an $\mathcal{F}$-group. Then: 
(1) there exists a complete canonical Hom $(G, H)$-diagram;

(2) if $H$ is a fixed subgroup of $G$ then there exists an $H$-complete canonical $\mathrm{Hom}_{H}(G, H)$-diagram.

\section{Some applications to equations in $\mathcal{F}$-groups}

Consider monomorphisms from $\mathcal{F}$-group $G$ to $\mathcal{F}$-group $H$. One can define an equivalence relation on the set of all such monomorphisms: two monomorphisms $\phi$ and $\psi$ are equivalent if $\psi$ is a composition of $\phi$ and conjugation by an element from $H$.

ThEOREm 15.1. Let $G[H]$ be an $\mathcal{F}$-group, and let $\mathcal{A}=\left\{A_{1}, \ldots, A_{n}\right\}[$ respectively, $\left.\mathcal{B}=\left\{B_{1}, \ldots, B_{n}\right\}\right]$ be a finite set of non-conjugated maximal abelian subgroups of $G$ [respectively, $H]$ such that the abelian decomposition of $G$ modulo $\mathcal{A}$ is trivial. The number of equivalence classes of monomorphisms from $G$ to $H$ that map subgroups from $\mathcal{A}$ onto conjugates of the corresponding subgroups from $\mathcal{B}$ is finite. A set of representatives of the equivalence classes can be effectively found.

Corollary 15.2. Let $G$ be an $\mathcal{F}$-group, and let $\mathcal{A}=\left\{A_{1}, \ldots, A_{n}\right\}$ be a finite set of maximal abelian subgroups of $G$. Denote by Out $(G ; \mathcal{A})$ the set of those outer automorphisms of $G$ which map each $A_{i} \in \mathcal{A}$ onto a conjugate of it. If $\operatorname{Out}(G ; \mathcal{A})$ is infinite, then $G$ has a non-trivial abelian splitting, where each subgroup in $\mathcal{A}$ is elliptic. There is an algorithm to decide whether $\operatorname{Out}(G ; \mathcal{A})$ is finite or infinite. If $\operatorname{Out}(G ; \mathcal{A})$ is infinite, the algorithm finds the splitting. If Out $(G ; \mathcal{A})$ is finite, the algorithm finds all its elements.

Let $G, H, \mathcal{A}, \mathcal{B}$ be as in the formulation of the theorem. Let $G=\left\langle x_{1}, \ldots, x_{k} \mid r_{1}\left(x_{1}, \ldots, x_{k}\right)=1, \ldots, r_{\ell}\left(x_{1}, \ldots, x_{k}\right)=1\right\rangle$. Let $A_{i}=\left\langle a_{i 1}, \ldots, a_{i k_{i}}\right\rangle$, $B_{i}=\left\langle b_{i 1}, \ldots, b_{i k_{i}}\right\rangle, i=1, \ldots, k_{i}$. Let $C_{i}$ be a cyclic group generated by $c_{i}$ if $A_{i}$ is cyclic, and free abelian group generated by $c_{i 1}, \ldots, c_{i, 2 k_{i}}$ otherwise.

LEMma 15.3. Let $G, H, \mathcal{A}, \mathcal{B}$ be as in the formulation of the theorem, $C_{1}, \ldots, C_{n}$ be free abelian groups as above. Let

$$
\begin{gathered}
K=\left\langle G, H, C_{1}, \ldots, C_{n}, t_{1}, \ldots, t_{n}\right| t_{n}=1, a_{i 1}^{t_{i}}=c_{i}=b_{i 1}, \text { if } k_{i}=1, \\
\left.a_{i j}^{t_{i}}=c_{i, k_{i}+j}, b_{i j}=c_{i j}, \text { if } k_{i} \neq 1, i=1, \ldots, n, j=1, \ldots, k_{i}\right\rangle .
\end{gathered}
$$

Then the reduced graph of groups $\Delta$ that corresponds to the given presentation of $K$ is an abelian JSJ decomposition of $K$ modulo $H$.

Proof. Let $\tilde{\Delta}$ be the Bass-Serre tree corresponding to $\Delta$, and let $Y$ be a minimal $K$-tree. Assume that $H \subset K$ is elliptic when acting on $Y$. It follows that $B_{i}$ is conjugated to an elliptic subgroup, therefore is an elliptic subgroup itself, for each $i$. Therefore each $C_{i}$ and $A_{i}$ is an elliptic subgroup. Now, suppose that $G$ is not elliptic when acting on $Y$. In the non-trivial splitting that $G$ inherits from its action on $Y$, each subgroup $A_{i}$ is elliptic, a contradiction to our assumption that the decomposition of $G$ relative to $\mathcal{A}$ is trivial. Hence, $G$ is elliptic when acting on $Y$. Therefore, the vertex $v$ (or $w_{i}$ ) fixed by $G$ [or $C_{i}$ ] in $\tilde{\Delta}$ can be mapped to a vertex fixed by $G$ [or $C_{i}$ ] in $Y$. If $y \in Y$ (or $y_{i} \in Y$ ) is a vertex fixed by $G$ [or $C_{i}$ ], then the edge joining $v$ and $t_{i} \cdot w_{i}$ can be mapped to the path joining $y$ and the vertex fixed by $t_{i} C_{i} t_{i}^{-1}$. Thus, we have defined a simplicial map from the extended fundamental domain of $\tilde{\Delta}$ to $Y$. Extend this map equivariantly to obtain 
a simplicial map from $\tilde{\Delta}$ into $Y$. Since $Y$ is a minimal $G$-tree, this latter map is onto; the claim follows.

PROOF OF THE THEOREM.

Consider the following system $W=1$ of equations in variables $x_{1}, \ldots, x_{k}, y_{1}, \ldots, y_{n}$ over $H$ :

$$
\begin{gathered}
r_{1}\left(x_{1}, \ldots, x_{k}\right)=1, \ldots, r_{\ell}\left(x_{1}, \ldots, x_{k}\right)=1, a_{i 1}^{y_{i}}=b_{i 1}, \text { if } k_{i}=1, \\
{\left[a_{i j}^{y_{i}}, b_{i s}\right]=1, \text { if } k_{i} \neq 1, i=1, \ldots, n, y_{n}=1,}
\end{gathered}
$$

where $a_{i 1}, \ldots, a_{i k_{i}}$ are generators of the subgroup $A_{i}$ of $G$ written in variables $x_{1}, \ldots, x_{k}$, and $b_{i 1}, \ldots, b_{i k_{i}}$ are generators of the subgroup $B_{i}$ of $H$ written in variables $h_{1}, \ldots, h_{m}$. If the system $W=1$ is irreducible, then $K=H_{R(W)}$. If $W=1$ is not irreducible, then $V(W)$ is a union of irreducible subvarieties over $H$. Denote by $W_{1}=1, \ldots, W_{t}=1$ the systems that define these subvarieties. Suppose that there is $W_{j}=1$ such that the canonical $H$-homomorphism from $H_{R(W)}$ onto $H_{R\left(W_{i}\right)}$ is a monomorphism on $G$ (we can verify this effectively). Since we will consider systems $W_{j}=1$ independently, we skip the index now and will write $W=1$ instead of $W_{j}=1$. Let the subgroups $C_{i}$ be mapped into free abelian subgroups $\bar{C}_{i}=\left\langle c_{i 1}, \ldots, c_{i t_{i}}\right\rangle$, and let $Z_{i}=\left\langle z_{i 1}, \ldots, z_{i k_{i}}\right\rangle$ be the image of $A_{i}$ in $\bar{C}_{i}$. Notice that $Z_{i}$ and $B_{i}$ generate $\bar{C}_{i}$. Then $H_{R(W)}$ has the following presentation:

$$
\left\langle G, H, \bar{C}_{1}, \ldots, \bar{C}_{n}, t_{1}, \ldots, t_{n} \mid t_{n}=1, a_{i j}^{t_{i}}=z_{i j}, b_{i j}=c_{i j}, i=1, \ldots, n, j=1, \ldots, k_{i}\right\rangle .
$$

Indeed, this group is embedded into a series of extensions of centralizers of the image of $K$ in $H_{R\left(W_{j}\right)}$, therefore it is fully residually free and is itself $H_{R\left(W_{j}\right)}$.

Similarly to Lemma 15.3 one can show that the reduced graph of groups $\Delta$ that corresponds to the given presentation of $H_{R(W)}$ is a JSJ decomposition of $H_{R(W)}$ modulo $H$.

The group $H$ can be canonically embedded into an NTQ group $N$ (see Section 9). One can define a free regular Lyndon's length function from $N$ onto $\Lambda=Z^{m}$ which is also a free length function on $H$ (Section 12). Denote this length function by $\ell$.

Consider a parametric generalized equation $\Omega$ for $W=1$ with $H$-bases as parameters. We consider only those generalized equations $\Omega$ for which $G \leq F_{R(\Omega)} \leq$ $N_{R(\Omega)}$. Construct the tree $T(\Omega)$ as described in Subsection 5.3 with respect to the length function $\ell$. The tree will be the same as in Section [13.1] It is enough to trace possible paths in $T(\Omega)$ for a minimal solution of $W=1$ such that the values of $y_{i}$ are commensurable with the value of maximal elements in $B_{i}$. Indeed, when we take a minimal solution we only change values of $y_{i}$ and conjugate $G$. Denote the subtree corresponding to these paths by $T^{\prime}(\Omega)$. In this case, constructing the subtree $T^{\prime}(\Omega)$, we do not have to consider infinite branches corresponding to Cases 15 and 12. Indeed, even if $F_{R(\Omega)}$ contains a $\mathrm{QH}$ subgroup $Q$, by Lemma 2.13 the group $H_{R(W)}$ is conjugated into some subgroup $P_{i}$ from Lemma 2.11, therefore we can consider only minimal solutions of $\Omega$ with respect to $Q$. If, reducing $\Omega$ to the terminal equations we obtain some free variables because the boundary between the variables $h_{i}$ and $h_{i+1}$ does not touch any base, we can consider $\operatorname{ker}(\Omega)$ instead of $\Omega$. Indeed, in this case $\bar{H}_{R(W)}$ is embedded into $F_{R(k e r(\Omega))}$. Therefore Cases 7-10 ( Subsection [5.3) can only appear a bounded number of times in the subtree $T^{\prime}(\Omega)$ corresponding to the actual paths for a minimal solution. Hence this subtree is finite. 
In the leaf vertices $v$ of $T^{\prime}(\Omega)$ we obtain generalized equations of four types:

1) generalized equations $\Omega_{v}$ with intervals labelled by generators $h_{1}, \ldots, h_{m}$ of $H$;

2) generalized equations $\Omega_{v}$ such that $F_{R\left(\Omega_{v}\right)}$ is a proper quotient of $F_{R(\Omega)}$, and the image of $G$ in $F_{R\left(\Omega_{v}\right)}$ is a proper quotient of $G$;

3) generalized equations $\Omega_{v}$ such that $F_{R\left(\Omega_{v}\right)}$ is a proper quotient of $F_{R(\Omega)}$, and the image of $G$ in $F_{R\left(\Omega_{v}\right)}$ is isomorphic to $G$;

4) generalized equations $\Omega_{v}$ with vertex $v$ of type 2 , such that the solution can be taken minimal with respect to all the periodic structures.

Therefore in the terminal vertices of $T_{\mathrm{dec}}^{\prime}(\Omega)$ ( Subsection 7.5) we only have Cases (1)-(3).

In Case 1) we solved the generalized equation $\Omega$ in $N$, and we just have to check whether the image of $G$ belongs to $H$ and is isomorphic to $G$.

In Case 2) we cannot have a monomorphism from $G$ to $H$, so we do not continue.

If we have Case 3) we apply the leaf-extension transformation ( Subsection 9.1) at this leaf vertex. Let $\bar{K}$ be the image of $H_{R(W)}$ in $F_{R(\Omega)}$. The group $\bar{K}$ is freely indecomposable modulo $H$. Moreover, a JSJ decomposition of $\bar{K}$ modulo $H$ either has two vertices stabilized by $G$ and $H$ and vertices with abelian vertex groups that are images of $\bar{C}_{1}, \ldots, \bar{C}_{n}$ or has one vertex with the stabilizer containing $H$ and the image of $G$ and some vertices with abelian vertex groups. Therefore, we again only consider minimal (and commensurable with elements of edge groups in the decomposition of $\bar{K}$ ) solutions of $\Omega_{v}$ with respect to the groups of canonical automorphisms. The number of times when we have case 3 ) is finite. Therefore after a finite number of steps we either show that there are no solutions of $W=1$ which are monomorphisms on $G$ or end up with Case 1).

We now check which solutions of $W=1$ in $N$ belong to $H$. This can be done effectively by Theorem 3.12

\section{References}

[1] S.I. Adian, Burnside problem and identities in groups, Science, Moscow, 1975.

[2] K.I.Appel, One-variable equations in free groups, Proc. Amer. Math. Soc., 19 (1968), 912918.

[3] H. Bass, Groups acting on non-archimedian trees, Arboreal group theory, 1991, 69-130.

[4] G. Baumslag, A. Miasnikov, V. Remeslennikov, Algebraic geometry over groups I. Algebraic sets and ideal theory, Journal of Algebra, 219 (1999), 16-79.

[5] Baumslag G., Myasnikov A., Remeslennikov V., Residually hyperbolic groups, Proc. Inst. Appl. Math. Russian Acad. Sci., 24 (1995), 3-37.

[6] G. Baumslag, On generalized free products, Math. Zeitschr., 7, no. 8 (1962), 423-438.

[7] R. Bryant, The verbal topology of a group, Journal of Algebra, 48 (1977), 340-346.

[8] M. Bestvina, M. Feighn, Stable actions of groups on real trees. Invent. Math., 121, no. 2 (1995), 287-321.

[9] M. Bestvina, M. Feighn, Bounding the complexity of simplicial group actions, Invent. Math., 103 (1991), 449-469.

[10] B.H. Bowditch, Cut points and canonical splittings of hyperbolic groups, Acta.Math., 180 (1998), 145-186.

[11] V. K. Bulitko, Equations and inequalities in a free group and a free semigroup (Russian), Tul. Gos. Ped. Inst. U cen. Zap. Mat. Kaf. no. 2 Geometr. i Algebra, (1970), 242-252.

[12] I. Bumagin, The conjugacy problem for relatively hyperbolic groups, Algebraic and Geometric Topology, to appear.

[13] C.C. Chang and H.J. Keisler, Model Theory, North-Holland, London, N.Y, 1973.

[14] C. Champetier and V.Guirardel Limit groups as limits of free groups: compactifying the set of free groups Preprint. 
[15] I. Chiswell, Abstract length functions in groups. Math. Proc. Cambridge Philos. Soc., 80, no. 3 (1976), 451-463.

[16] D. E. Cohen, Combinatorial group theory: a topological approach, Cambridge Univ. Press, 1989.

[17] L.P. Comerford and C.C. Edmunds. Quadratic equations over free groups and free products, Journal of Algebra, 68 (1981), 276-297.

[18] L. P. Comerford Jr. and C. C. Edmunds, Solutions of equations in free groups, Walter de Gruyter, Berlin, New York, 1989.

[19] M.J. Dunwoody and M.E. Sageev, JSJ splittings for finitely presented groups over slender subgroups,Invent. Math. 135 (1999), 25-44.

[20] F.Dahmani, Combination of convergence groups, Geom. Topol. 7 (2003) 933-963.

[21] Yu. L. Ershov and E. A. Palutin, Mathematical Logic, Walter de Gruyter, Berlin, New York, 1989.

[22] K. Fujiwara and P. Papasoglu, JSJ decompositions of finitely presented groups and complexes of groups,Preprint, 1997.

[23] Gaboriau D., Levitt G., Paulin F., Pseudogroups of isometries of $R$ and Rips' theorem on free actions on R-trees. Israel J. Math., 87 (1994), 403-428.

[24] D. Gildenhuys, O. Kharlampovich and A. Myasnikov, CSA groups and separated free constructions, Bull. Austral. Math. Soc., 52 (1995), 63-84.

[25] R. I. Grigorchuk and P. F. Kurchanov, Some questions of group theory connected with geometry, In Encyclopedia of mathematics, 1989.

[26] R.I. Grigorchuk and P.F. Kurchanov, On quadratic equations in free groups, Contemp. Math., 131(1) (1992), 159-171.

[27] V. Guba, Equivalence of infinite systems of equations in free groups and semigroups to finite subsystems, Mat. Zametki, 40 (1986), 321-324.

[28] A. Hoare, A. Karrass, D.Solitar, Subgroups of finite index of Fuchsian groups, Math. Z., 120 (1971), 289-298.

[29] A. Hoare, A. Karrass, D.Solitar, Subgroups of finite index of Fuchsian groups, Math. Z., 125 (1972), 59-69.

[30] K. Johannson, Homotopy equivalences of 3-manifolds with boundaries,Lecture Notes in Math., 761, Springer-Verlag, Berlin-New York, 1979.

[31] W.H. Jaco and P.B. Shalen, Seifert fibered spaces in 3-manifolds, Mem. Amer. Math. Soc., 220, Amer. Math. Soc., Providence, RI, 1979.

[32] I. Kapovich, R. Weidmann and A. Myasnikov, Foldings, graphs of groups and the membership problem, to appear in IJAC.

[33] O. Kharlampovich and A. Myasnikov. Hyperbolic groups and free constructions Transactions of Math., 350, no. 2 (1998), 571-613.

[34] O. Kharlampovich and A. Myasnikov, Description of Fully Residually Free Groups and Irreducible Affine Varieties Over a Free Group Center de Recherchers Matematiques, CRM Proceedings and Lecture Notes, 17 (1999), 71-80.

[35] O. Kharlampovich and A. Myasnikov, Irreducible affine varieties over a free group. 1: irreducibility of quadratic equations and Nullstellensatz, J. of Algebra, 200 (1998), 472-516.

[36] O. Kharlampovich and A. Myasnikov, Irreducible affine varieties over a free group. II: Systems in triangular quasi-quadratic form and description of residually free groups, J. of Algebra, 200, no. 2 (1998), 517-570.

[37] O. Kharlampovich and A. Myasnikov, Tarski' problem about the elementary theory of free nonabelian groups has a positive solution, ERA-AMS, 4 (1998), 101-108.

[38] O. Kharlampovich and A. Myasnikov, Implicit function theorem over free groups and genus problem, AMS/IP Studies in Advanced Mathematics, 24 (2001), 77-83.

[39] O. Kharlampovich and A. Myasnikov, Elementary theory of free non-abelian groups, McGill University Preprint, 1999, HTTP://www.math.mcgill.ca/olga/publications.html

[40] O. Kharlampovich and A. Myasnikov, Implicit function theorem over free groups, McGill University Preprint, 1999, HTTP://www.math.mcgill.ca/olga/publications.html

[41] O. Kharlampovich, Equations over fully residually free groups, McGill University Preprint, 1999, HTTP://www.math.mcgill.ca/olga/publications.html

[42] O. Kharlampovich, A. Myasnikov, D. Serbin, Equations over fully residually free groups, Preprint, 2002 
[43] O. Kharlampovich, A. Myasnikov, D. Serbin, Free regular length functions on fully residually free groups, Preprint, 2004.

[44] O. Kharlampovich and A. Myasnikov, Equations in a free Q-group, Transactions of the AMS, 350, no. 3 (1998), 947-974.

[45] O. Kharlampovich and A. Myasnikov, Algebraic geometry over free groups: Lifting solutions into generic points, this volume.

[46] O. Kharlampovich, A. Myasnikov, V. Remeslennikov, D. Serbin, Subgroups of fully residually free groups: algorithmic problems, Group theory, Statistics and Cryptography ( A.G. Myasnikov and V. Shpilrain, editors), Contemp. Math., Amer. Math. Soc., 360 (2004).

[47] R.C. Lyndon, Groups with parametric exponents, Trans. Amer. Math. Soc., 96 (1960), 518533.

[48] R.C. Lyndon, Length functions in groups, Math. Scand., 12 (1963), 209-234.

[49] R.C. Lyndon and P.E. Schupp, Combinatorial group theory, Springer, 1977.

[50] W. Magnus, A. Karras, D. Solitar, Combinatorial group theory, Interscience Publ., 1966.

[51] G.S. Makanin. Equations in a free group (Russian), Izv. Akad. Nauk SSSR, Ser. Mat., 46 (1982), 1199-1273. transl. in Math. USSR Izv., 21, (1983); MR 84m:20040.

[52] G.S. Makanin, Decidability of the universal and positive theories of a free group (Russian), Izv. Akad. Nauk SSSR, Ser. Mat., 48, no. 1 (1985), 735-749. transl. in Math. USSR Izv., 25 (1985), MR 86c:03009.

[53] A. Myasnikov, V. Remeslennikov, D. Serbin, Regular free length functions on Lyndon's free $\mathbb{Z}[t]$-group $F^{\mathbb{Z}[t]}$, this volume.

[54] A. Myasnikov and A. Kvaschuk, Big powers and free constructions, International Journal of Algebra and Computation, to appear.

[55] A. G. Myasnikov and V. N. Remeslennikov, Exponential groups 2: extension of centralizers and tensor completion of csa-groups, International Journal of Algebra and Computation, 6 , no. 6 (1996), 687-711.

[56] A. Myasnikov and V. Remeslennikov, Length functions on free exponential groups, Proc. Russian Acad. of Sci., Siberian Division, Inst. of Appl. Math., 26 (1996), 3-33.

[57] A. Myasnikov, V. Remeslennikov and D. Serbin, Fully residually free groups and graphs labeled by infinite words, to appear in Internat. J. Algebra and Comput.

[58] A. Yu. Ol'shanskii, On residualing homomorphisms and G-subgroups of hyperbolic groups, , Int. J. Algebra and Comput. 3, no. 4 (1993), 365-409.

[59] F. Paulin, Outer automorphisms of hyperbolic groups and small actions on $R$-trees, in Arboreal group theory (R. C. Alperin, ed.), MSRI Publications, 19 (1991), 331-343.

[60] A. Razborov, On systems of equations in a free group, Math. USSR, Izvestiya, 25, no. 1 (1985), 115-162.

[61] A. Razborov, On systems of equations in free groups, In Combinatorial and geometric group theory. Edinburgh 1993, Cambridge University Press, 1995, 269-283.

[62] A. Razborov, On systems of equations in a free group, PhD thesis, Steklov Math. Institute, Moscow, 1987.

[63] Z. Sela, Structure and rigidity in (Gromov) hyperbolic groups and discrete groups in rank 1 Lie groups, II, Geom Funct. Anal., 7 (1997), 561-593.

[64] R. Swan Groups of Cohomological Dimension One, J. of Algebra, 12 (1969), 585-601.

[65] Serre, J.-P., Trees. Translated from the French original by John Stillwell,Springer-Verlag, Berlin-New York, 1980.

[66] V.N. Remeslennikov, E-free groups, Siberian Math. J., 30, no. 6 (1989), 153-157.

[67] E. Rips and Z. Sela, Cyclic splittings of finitely presented groups and the canonical JSJ decomposition, Annals of Math., 146 (1997), 53-109.

[68] Z. Sela, Acylindrical accessibility for groups Inv. Math., 129 (1997), 527-275.

[69] Z. Sela, Diophantine geometry over groups I: Makanin-Razborov diagrams,Publications Mathematiques de l'IHES 93 (2001), 31-105. 
Department of Mathematics and Statistics, McGill University, Montreal, QC, CANADA, H3A2K6

E-mail address: olga@math.mcgill.ca

Department of Mathematics and Statistics, McGill University, Montreal, QC, CANADA, H3A2K6

E-mail address: alexeim@att.net 